\title{
A PRONY ALGORITHM FOR SHALLOW WATER WAVEGUIDE
}

ANALYSIS

by

Ferdinand Joseph Diemer

BSEE, United States Naval Academy (1980)

SUBMITTED IN PARTIAL FULFILLMENT OF THE REQUIREMENTS FOR THE DEGREE OF

OCEAN ENGINEER

at the

MASSACHUSETTS INSTITUTE OF TECHNOLOGY

and the

WOODS HOLE OCEANOGRAPHIC INŚTTTUTION

August, 1987

(c) Ferdinand Joseph Diemer, 1987

The author hereby grants M.I.T. and WHOI permission to reproduce and to distribute copies of this thesis document in whole or in part.

Signature of author

Joint Program in Oteanographic Engineering

Massachusetts Institute of Technology and

Woods Hole Oceanographic Institution

Ceritified by

George Frisk, Thesis Supervisor

Accepted by

George V. Fris/, Chairman, Joint Committee

for Oceanographic Engineering, Massachusetts Insititute of Technology/Woods Hole Oceanographic Institution 


\title{
A Prony Algorithm for Shallow Water Waveguide Analysis
}

\author{
Ferdinand Joseph Diemer
}

Submitted in partial fulfillment of the requirements for the degree of

Ocean Engineer

at the

Massachusetts Institute of Technology

and the

Woods Hole Oceanographic Insititution

August 1987

\begin{abstract}
Low frequency acoustic propagation in shallow water is examined from a normal mode context. By modelling the far field pressure field as a modal sum, propagating mode characteristics of wavenumber, initial phase, attenuation and amplitude may be estimated using a high resolution parameter modeling technique. The advantages of such an algorithm are the resolution of closely spaced modes in a range independent environment and the ability to analyze range dependent waveguides.

This thesis presents the application of a Prony algorithm to the shallow water environment. The algorithm operates directly on the signal matrix. Synthetically generated, range independent pressure fields are used to analyze the technique's performance and to observe its sensitivity to variations in model specifications. Noise is added to determine the threshold of acceptable performance. As a consequence of field data tests, further enhancements to the algorithm are suggested.

Range dependent performance is evaluated on a coastal wedge example and geoacoustic parameter shift example.

Thesis advisor: George V. Frisk, Associate Scientist, Woods Hole Oceanographic Institution
\end{abstract}




\section{Acknowledgements}

My two years as a graduate student have passed swiftly by; it has been an enjoyable and rewarding period. The highlights of this experience have been the aquaintances made in the MIT/WHOI community. While there are far too many people who have enhanced my work to recognize here, certain individuals deserve special mention.

To my advisor, George Frisk, a very special thanks for his dedication, support and enthusiasm. His insight and guidance were always evident along with an extremely positive attitude toward the research effort. Thanks also to Al Oppenheim, Victor Zue, Jim Lynch, Subramanian Rajan, Andy Kurkjian and Mike Wengrovitz for their suggestions, comments and availability, to Arthur Newhall and Maxine Jones for their programming talents and assistance, and to Mary Athanis, Denise Cormier and Marilyn Staruch for adding a touch of reality to the academic world.

Among my compatriots in the MTT/WHOI community, there have been many who have contributed to this research through discussions and recommendations. They include Paul Bushong, Kevin Casey, Peter Dahl, Jim Miller, Jim Riley, Nitin, Nick Makris, Hee Seong and $=$ Rob Fricke.

This opportunity to participate in graduate studies was made possible through the Secretary of the Navy's Program in Oceanography and Oceanographic Engineering and RADM Seesholtz, USN, Oceanographer of the Navy, as well as through the efforts of Art Baggeroer, Bob Spindel, Charlie Hollister and Jake Peirson among others. In addition to my recognition of their support, I'd like to thank RADM W.A. Owens, USN, for his guidance and concern in my personal goals.

Finally, a very belated thanks to Darlene and my family for their support, love and assistance throughout the years. 


\section{Table of Contents}

Chapter 1 - Introduction $\quad 5$

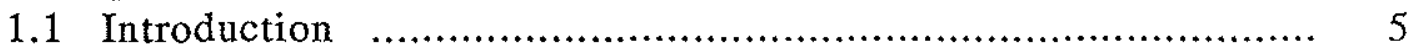

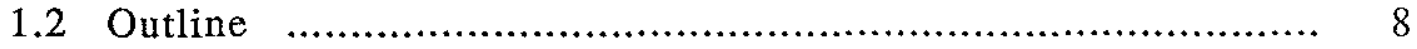

Chapter 2 - Basic Theory 9

2.1 Normal Mode Theory Review.......................................... 9

2.2 Prony's Method..................................................... 17

2.3 Application of Prony's Method to Shallow Water Waveguide................ 28

2.4 Summary......................................................... 38

Chapter 3 - Prony Method Evaluation $\quad 39$

3.1 Depth Dependent Green's Function...................................... 39

3.2 Prony Energy Spectral Density......................................... 44

3.3 Algorithm Perfomance in the Range Independent Waveguide................ $\quad 50$

3.4 Prony's Method in a Noisy Environment................................ 74

3.5 Summary........................................................ 105

Chapter 4 - Range Dependent Waveguides 107

4.1 Range Dependent Performance........................................ 107

4.2 Ramp Example................................................... 111

4.3 Geoacoustic Parameter Shift Example................................... 122

4.4 Summary ....................................................... 133

$\begin{array}{ll}\text { Chapter } 5 \text { - Conclusions } & 134\end{array}$

5.1 Recommendations and Future Considerations........................... 134

5.2 Conclusions............................................................. 149

Appendix A Essentials of Sturm Liouville Problems................................. 151

Appendix B Some Signal Processing Basics................................... 153

Appendix C PRAWNS Program Listing...................................... 156

Bibliograph y ....................................................... 182 


\section{Chapter 1 Introduction}

\subsection{Introduction}

In this thesis, we shall investigate the application of a signal processing technique to acoustic propagation in a shallow water waveguide. As is the case in many disciplines, advances in the ocean acoustics community tend to occur in incremental steps. Often, the approaches used in acoustic problem formulation or analysis are successfully applied in other research efforts. Examples include ray theory (from optics), method of images (electromagnetic theory) and fast field programs (digital signal processing). We shall examine the application of Prony's method to the problem of resolving normal modes in $\mathrm{CW}$ data obtained on a horizontal array in shallow water. We expect to formulate a technique which requires the researcher to incorporate knowledge of the acoustic propagation into a signal modelling problem. In return for this bounding or constraint of the problem, we anticipate a gain in two areas. First, in the range independent waveguide, Prony's method should allow the resolution of closely spaced propagating modes. Second, in a range dependent waveguide, the short apertures used by the high resolution techniques may permit estimation of waveguide parameters by an adiabatic assumption.

To obtain such high resolution using conventional discrete Hankel transforms and FFT beamforming techniques requires a large aperture since the resolution is inversely proportional to the length of the array[1]. In theoretical or computer generated fields, this length of data may be easily obtained. Shallow water data rarely meets this criterion since ocean waveguides which are invariant over an interval of kilometers (which is needed to obtain the desired resolution) are not generally found in practice.

Applications of Prony's method may be found in a variety of disciplines. Research examples include seismic exploration[2], acoustic echo reduction[3], subsurface radar[4], 
beamforming[5], and structural analysis[6] . The specific use of Prony's method in a shallow water waveguide modal context has been explored in parallel with this thesis development by Shang, et al.[7]. The Shang study considers the algorithm in a scheme to localize a source and provide some waveguide characterization. Their characterization approach differs from this thesis in order selection, filter coefficient determination and attenuation parameter estimation. Such differences in implementation emphasizes the flexible nature of algorithm structure within the framework of Prony's method.

The motivation for exploring this approach is to assist in the effort to solve the inverse problem of determining geoacoustic parameters. Specifically, the geoacoustic parameter inverse technique requires the set of horizontal wavenumbers of the propagating modes as input data[8-11]. The signal processing method used provides a means to identify the horizontal wavenumber of an acoustic field. Ultimately, we envision measurements of the pressure field in the water column yielding a set of wavenumbers which may then be used to infer bottom properties.

There are two sets of field data which have been collected to date. These provide a test environment of the algorithm in the real world. The first set was collected in May of 1984 off the coast of Nantucket Island, MA at $140 \mathrm{~Hz}$ and $220 \mathrm{~Hz}[10]$ and the other set was obtained in September of 1985 off the coast of Corpus Christi, TX at $50 \mathrm{~Hz}$ and $140 \mathrm{~Hz}[12]$. These sites were selected since the bottoms are reasonably flat and other studies of bottom properties are available for comparison of the geoacoustic parameters. The experimental setup uses a small vessel which tows a two frequency CW source at a fixed depth[8]. The source is towed away from two fixed moored receivers and the horizontal source-receiver range is monitored via a radar tracking system. The data collection technique consists of drifting away from the receivers. The drift rate must be low ( $<0.5$ knots) to provide adequate sampling (to avoid aliasing) of the pressure field. The hydrophones are part of a larger BODIS assembly which removes harmonic time dependence of the pressure data by quadrature demodulation. 
The effect of the drift scheme and quadrature demodulation is to model the data set as being collected on a synthetic array. The physical setup is summarized in figure 1.1.1.

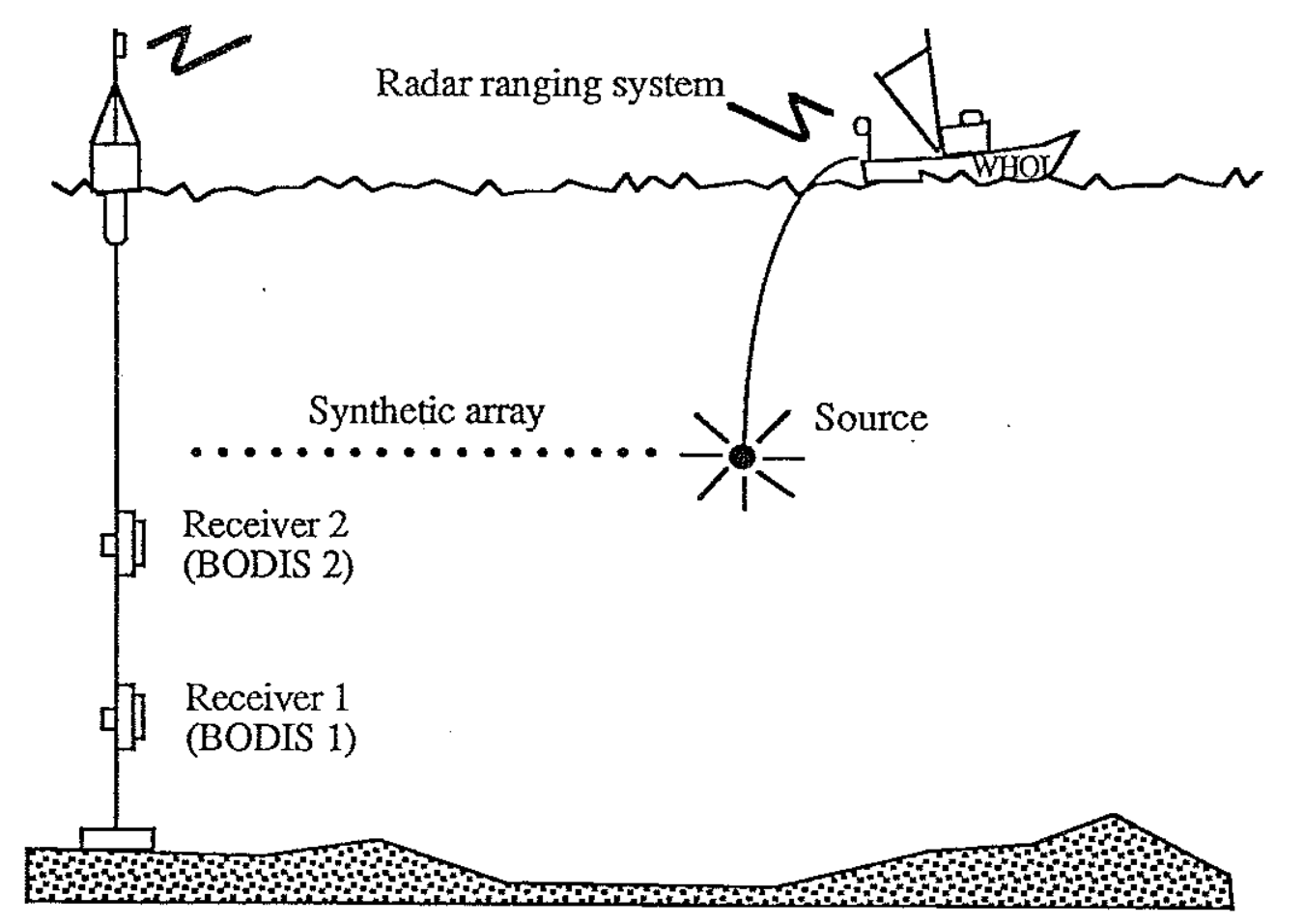

Fig 1.1.1 Experimental setup for data collection 


\subsection{Outline}

Chapter 2 provides a basic review of normal mode theory in an acoustic waveguide by developing the mathematical description in terms of a Sturm Liouville problem. The second section provides a derivation of the Prony model and explores the nuances of the algorithm used. The last part of the chapter casts the normal mode environment in terms of the model and illustrates its validity. Two examples are provided: the first involves the application of the method to synthetic pressure field data, while the second demonstrates the ability of the technique to extract mode shapes through use of a vertical array.

In Chapter 3, the depth dependent Green's function is reviewed and the Prony energy spectral density (ESD) is introduced. The ESD is used as a tool to transform all parameter estimates into a simple graphical display. As such, it acts as a tool to aid in waveguide analysis. Additional tools are developed and examined, and the algorithm is tested using these tools on two synthetically generated sets of data. The effect of changes in input variables on parameter estimates is examined and ranked according to sensitivity. This ranking leads to the development of a set of empirically derived guidelines to specify a model order, aperture size and averaging. The chapter concludes with a performance evaluation on field data.

Range dependent performance is addressed in Chapter 4. Two examples of a rangedependent environment are provided. One waveguide consists of an upslope propagation in a coastal wedge scenario. The second contains a step change in bottom geoacoustic properties.

Chapter 5 provides a "wish list" of enhancements and considerations in further versions of this high resolution scheme. Topping the list is the issue of more robust performance in noisy environments. An example of a bandpass filter scheme to address this issue is also provided. 


\section{Chapter 2 Basic Theory}

\subsection{Normal Mode Theory Review}

A review of basic normal mode theory is important to emphasize assumptions made in the development of the description of the acoustic field and in the application of the Prony model to this environment. An advantage of the normal mode approach is that it allows us to build on previous results as the boundary conditions become more complex. It will be shown that this method makes the problem tractable by casting the linear, second order differential equation arising from the boundary value problem in terms of a SturmLiouville problem. This allows us to apply the rich existing mathematical theory for the subject.

In this thesis, sound propagation in shallow water will be treated as a field propagating within a waveguide constrained by the surface and the bottom. The field distribution within the waveguide is affected by the boundary characteristics so that the local modes act as a sampling mechanism for the properties of the boundaries. It is assumed that the top boundary condition remains constant (in this case, a pressure release surface) while the mode wavenumbers are affected by changes in the dimension of the waveguide (bathymetry) and bottom properties (such as sound speed, density and attenuation) (see fig 2.1.1). The water column is the region of interest for measurement purposes since the pressure field may be easily obtained by real towed or synthetic aperture arrays. In this section, we will assume that the wavegude is locally range-independent. After presenting some general normal mode theory, this section will use hard bottom and Pekeris waveguides to emphasize salient points. 


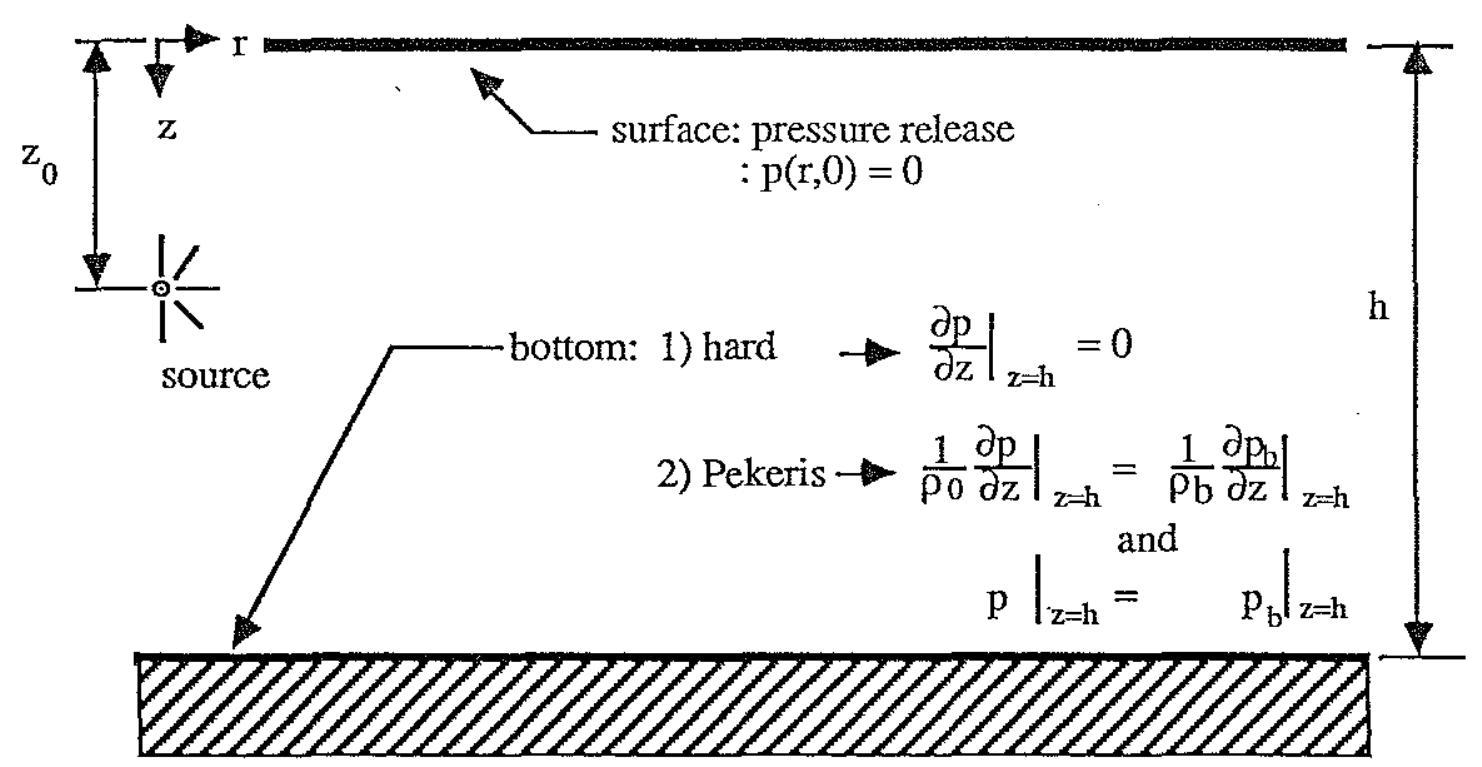

Fig 2.1.1. Shallow Water Waveguide Model

In the ocean acoustics waveguide, the governing equation for the acoustic pressure, $P$, is the time dependent wave equation[13]:

$$
\left(\nabla^{2}-\frac{1}{c^{2}(r)} \frac{\partial^{2}}{\partial t^{2}}\right) P(r, t)=-4 \pi F(r, t)
$$

where $F(r, t)$ is a source function. For the case of interest, we will consider a harmonic point source function. We therefore let $P(\underline{r}, t)=p(\underline{r}) e^{-j \omega t}$ and $F(\underline{r}, t)=f(\underline{r}) e^{-j \omega t}$ which transforms the wave equation to the inhomogeneous Helmholtz equation:

$$
\left(\nabla^{2}+k^{2}(r)\right) p(\underline{r})=-4 \pi f(r), k(r) \equiv \frac{\omega}{c(\underline{r})}
$$

The point source is modelled as an impulse function of strength $S$, ie:

$$
\left(\nabla^{2}+k^{2}(r)\right) p(\underline{r})=-4 \pi S \delta\left(\underline{r}-\underline{r}_{0}\right)
$$

In cylindrical coordinates, assuming horizontal stratification $\mathrm{k}(\mathrm{r})=\mathrm{k}(\mathrm{z})$ and a with $\phi(\mathrm{r})=$ $\mathrm{p}(\mathrm{r})$ and a source located at $\left(\mathrm{r}_{0}, \mathrm{z}_{0}, \theta_{0}\right)$, the Helmholtz equation is[14]:

$$
\frac{1}{\mathrm{r}} \frac{\partial}{\partial \mathrm{r}}\left(\mathrm{r} \frac{\partial \mathrm{p}}{\partial \mathrm{r}}\right)+\frac{1}{\mathrm{r}^{2}} \frac{\partial^{2} \mathrm{p}}{\partial \theta^{2}}+\frac{\partial^{2} \mathrm{p}}{\partial \mathrm{z}^{2}}+\mathrm{k}^{2}(\mathrm{z}) \mathrm{p}=-4 \pi \frac{\delta\left(\mathrm{r}-\mathrm{r}_{0}\right)}{\mathrm{r}} \delta\left(\mathrm{z}-\mathrm{z}_{\mathrm{n}}\right) \delta\left(\theta-\theta_{0}\right)
$$


Assuming cylindrical symmetry, $\mathrm{p}(\underline{\mathrm{r}})=\mathrm{p}(\mathrm{r}, \mathrm{z})$, and $\mathrm{r}_{0}=0$, the Helmholtz equation may be integrated with respect to $\theta$ to remove angular dependency:

$$
\int_{0}^{2 \pi}\left[\frac{1}{r} \frac{\partial}{\partial r}\left(r \frac{\partial p}{\partial r}\right)+\frac{\partial^{2} p}{\partial z^{2}}+k^{2}(z) p\right] d \theta=-4 \pi \frac{\delta(r)}{r} \delta\left(z-z_{0}\right)
$$

which leads to:

$$
\frac{1}{\mathrm{r}} \frac{\partial}{\partial \mathrm{r}}\left(\mathrm{r} \frac{\partial \mathrm{p}}{\partial \mathrm{r}}\right)+\frac{\partial^{2} \mathrm{p}}{\partial \mathrm{z}^{2}}+\mathrm{k}^{2}(\mathrm{z}) \mathrm{p}=-2 \frac{\delta(\mathrm{r})}{\mathrm{r}} \delta\left(\mathrm{z}-\mathrm{z}_{0}\right)
$$

The pressure field is constrained by the following boundary conditions:

- $p(r, 0)=0$ (pressure release surface)

- a bottom impedence boundary condition

and a Sommerfeld radiation condition (which specifies energy from the source as propagating outward)[14].

The two dimensional Green's function can be expanded in a complete orthonormal set of the eigenfunctions of depth, $z[15,16]$. The method of solving an inhomogeneous Sturm Liouville equation (see Appendix A) may be used as follows. First, the eigenfunctions, $\mathrm{u}_{\mathrm{n}}(\mathrm{z})$, are found by solving the homogeneous Helmholtz equation by separation of variables. This yields an equation for the $u_{n}(z)$ :

$$
\frac{\mathrm{d}^{2} \mathrm{u}}{\mathrm{dz^{2 }}}+\left[\mathrm{k}^{2}-\mathrm{k}_{\mathrm{n}}^{2}\right] \mathrm{u}=0 \text { and } \mathrm{k}_{\mathrm{n}}^{2} \text { is the separation constant. }
$$

The solution for $p$ is then assumed to have the form $p(r, z)=\sum_{n=1}^{\infty} R_{n}(r) u_{n}(z)$ and the assumed solution is substituted into equation (2.1.6), the inhomogeneous Helmholtz equation. Then, the orthogonality and completeness characteristics of the eigenfunctions, $u_{n}(z)$, are used to find the coefficients $R_{n}(r)$. For an arbitrary $k(z)$, the solution may be expressed as,

$$
p(r, z)=j \pi \sum_{n=1}^{\infty} u_{n}^{*}\left(z_{0}\right) u_{n}(z) H_{0}^{(1)}\left(k_{n} r\right)+I(r)
$$


in which $\mathrm{H}_{0}^{(1)}\left(\mathrm{k}_{\mathrm{n}} \mathrm{r}\right)$ is a Hankel function of the first type and is generated by the solution to equation (2.1.7). While the discrete sum corresponds to the trapped modes, the continuum contribution, I(r), consists of branch line integrals and "improper" modes (depending on the branch cut selection)[17-20].

Restricting our attention to the hard bottom case requires imposing a bottom boundary condition of $\left.\frac{\partial p}{\partial z}\right|_{z=h}=0$. The hard bottom isovelocity case yields an analytic solution for $\mathrm{u}_{\mathrm{n}}(\mathrm{z})[14]$ :

$$
\begin{aligned}
\mathrm{p}(\mathrm{r}, \mathrm{z})=\mathrm{j} \pi \sum_{\mathrm{n}=1}^{\infty} \sin \left(\gamma_{\mathrm{n}} \mathrm{z}_{0}\right) \sin \left(\gamma_{\mathrm{n}} \mathrm{z}\right) \mathrm{H}_{0}^{(1)}\left(\mathrm{k}_{\mathrm{n}} \mathrm{r}\right) \quad \text { where } \gamma_{\mathrm{n}} & =\sqrt{\mathrm{k}^{2}-\mathrm{k}_{\mathrm{n}}^{2}} \\
& =\text { vertical wavenumber } \\
& =\frac{\left(\mathrm{n}-\frac{1}{2}\right) \pi}{\mathrm{h}}
\end{aligned}
$$

Although a perfectly reflecting (hard) bottom is not found in nature, this simple model allows identification of three important normal mode characteristics which may be used in more complex models. First, as the range from the source increases, the Hankel function may be replaced by an asymptotic approximation[21]:

$$
\mathrm{H}_{0}^{(1)}\left(\mathrm{k}_{\mathrm{n}} \mathrm{r}\right) \approx \sqrt{\frac{2}{\pi \mathrm{k}_{n} \mathrm{r}}} \mathrm{e}^{\mathrm{j}\left(\mathrm{k}_{\mathrm{n}} \mathrm{r}-\frac{\pi}{4}\right)}
$$

Second, a propagating mode may be viewed as a cylindrically spreading, outgoing wave with a vertical shape determined by the mode eigenfunctions, $u_{n}(z)[22]$. Third, the placement of source and receiver directly affects mode amplitudes. In equations (2.1.8) and (2.1.9), we can see the product of the source and receiver eigenfunctions determine the mode excitation available at the receiver.

A more complex model introduced by Pekeris in 1948 consists of approximating the bottom by a half space with a constant density and sound speed[23]. The resulting 
eigenvalue spectrum consists of two seperate regions; one region of the spectrum has a discrete spectrum and the other region has a continuous spectrum. This change in spectrum characteristics is due to the bottom boundary defined as an impedence condition:

$$
\begin{aligned}
& \left.\frac{1}{\rho_{0}} \frac{\partial \mathrm{p}}{\partial \mathrm{z}}\right|_{\mathrm{z}=\mathrm{h}}=\left.\frac{1}{\rho_{\mathrm{b}}} \frac{\partial \mathrm{p}_{\mathrm{b}}}{\partial \mathrm{z}}\right|_{\mathrm{z}=\mathrm{h}} \text { and } \\
& \left.\left.\mathrm{p}\right|_{\mathrm{z}=\mathrm{h}=\mathrm{p}=\mathrm{b}}\right|_{\mathrm{z}=\mathrm{h}}
\end{aligned}
$$

The horizontal wavenumber which separates the discrete and continuous regions is known as the cutoff wavenumber is delineated by the wavenumber of the bottom half space, which has sound speed $c_{b}$ and density $\rho_{b}$. This discrete/continuous split spectrum is found in more complex models in which the horizontally stratified layers that make up the bottom profile are usually terminated at an arbitrary depth by an isovelocity halfspace. The cutoff wavenumber is set by this layer's wavenumber[24].

An intutive approach to cutoff is formulated by alternately posing the modal description as a superposition of up and down going plane waves(since $u_{n}(z)=\sin \left(\gamma_{n} z\right)=$ $\left.\frac{\text { ejmz }- \text { e-jmz }}{2 j}\right)[13,25]$. This expression is well suited to identifying modes which will propagate.

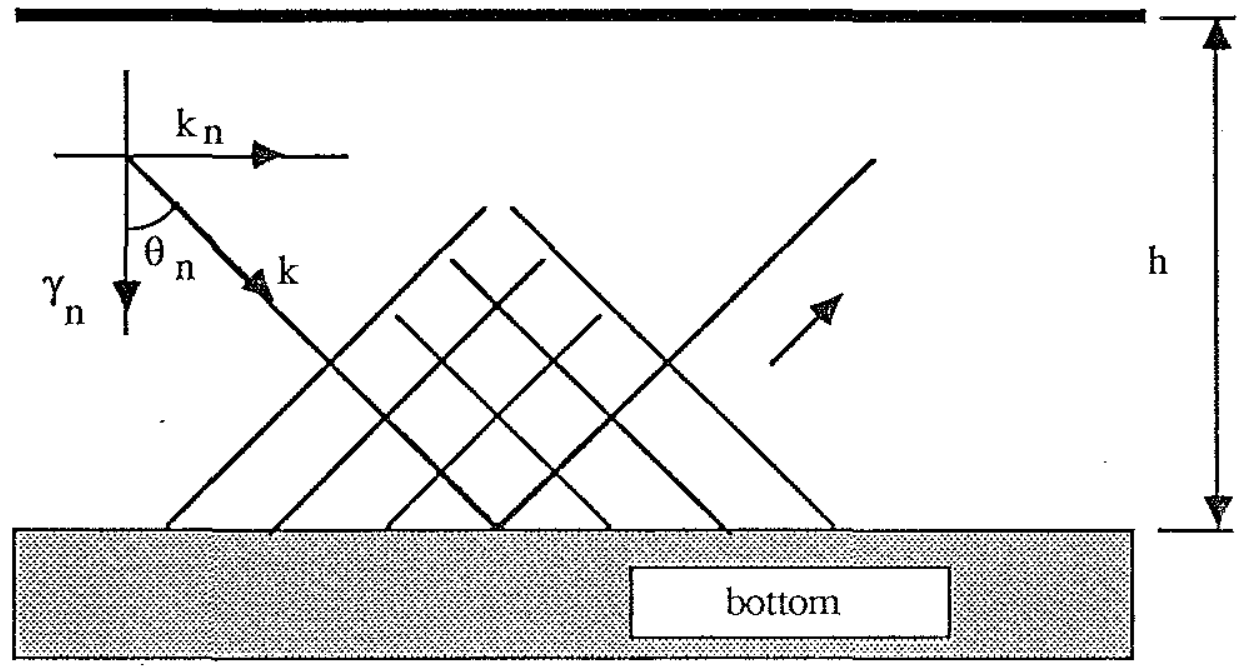

Fig 2.1.2 - Plane Waves Incident on Bottom 
As shown in figure 2.1.2, the downgoing plane wave is incident on the bottom at an angle $\theta_{n}$, which is defined by $\theta_{n}=\tan ^{-1}\left(\frac{k_{n}}{\gamma_{n}}\right)$. As the mode number, $n$, increases, the angle $\theta_{n}$ decreases and so the inclination of the plane waves becomes more nearly vertical. As $k_{n}$ approaches zero and becomes imaginary, the mode changes from a propagating mode to an exponentially decaying, inhomogeneous wave. This situation in which the sound energy is present as a heavily attenuated field is known as cutoff; the angle at which this occurs is known as the critical angle, $\theta c[26]$. An incident plane wave associated with a propagating mode will experience total reflection at the bottom; in the sediment, the sound pressure is exponentially damped (Fig 2.1.3).This "impedance condition" dictates the existence of a mechanism to account for energy "leaking" into the bottom since the plane waves (the modes are being modelled as a superposition of up and downgoing plane waves) incident on the bottom are no longer being perfectly reflected.

By decomposing the depth eigenfunctions into plane waves, the plane wave incident angles which lead to prepagating modes may be divided into two regions[27]:

- $\underline{\theta>\theta_{c}}$ : Region of perfect reflection resulting in a discrete set of trapped modes in water column and exponential decay in the bottom.

- $\underline{\theta<\theta_{c}}$ : Region known as the continuum which is propagation region where leaky (or virtual) modes can exist. These heavily attenuated modes allow energy to leak into the bottom; some of this energy may be directed back into the waveguide by the bottom's velocity/density profile. 


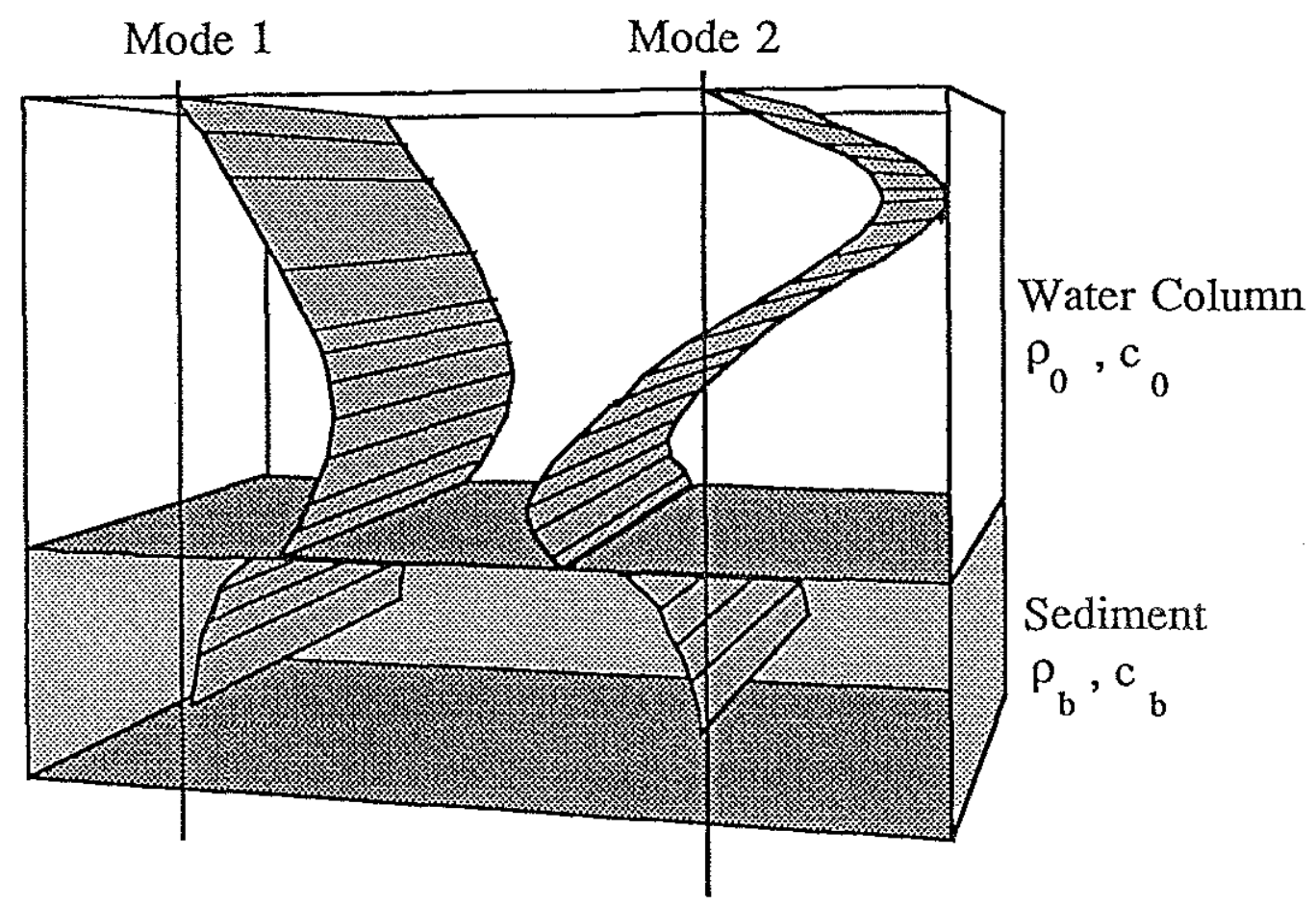

Fig 2.1.3 Pekeris Mode Shapes for Modes 1 and 2

The anticipated loss of amplitude with respect to range in a lossless waveguide should be $\frac{1}{\sqrt{\mathrm{r}}}$. This expected decrease is due to the cylindrical spreading term contained in the Hankel function. The observed rate of decrease is higher than this and is due to absorption in the water column and dissipation of energy by the sediment[26].

Mathematically, this may be treated by allowing the horizontal wavenumber to have a small imaginary component, ie $k_{n} \rightarrow k_{n}+j \delta k_{n}[28]$. By using this perturbative approach, some conclusions can be made regarding the effect of the attenuation on the modal sum description. The mode attenuation coefficient, $\delta \mathrm{k}_{\mathrm{n}}$, is proportional to the percentage of mode shape in the bottom. If the mode has significant amplitude in a lossy bottom there 
will be high attenuation with respect to range. While the water column mode shapes won't change when attenuation is taken into account, different modes will have different attenuation rates. This is evident if the plane wave decomposition of the modes approach is used. The higher order modes are incident on the bottom with steeper angles (closer to normal incidence). These modes undergo more reflections for a given horizontal range than modes which are incident closer to grazing.

The cursory review of normal mode theory of this section was meant to emphasize key aspects of the shallow water waveguide problem. The hard bottom waveguide example demonstrated the modal sum form. The asymptotic behavior of the Hankel function was stated as well as characteristics of the propagating energy[27]. The substitution of an impedance condition for the formerly hard bottom results in two distinct regions in the spectrum. In one, the spectrum is discrete and in the other, it is continuous. The distinction between the discrete and continuous spectrum may be set by the wavenumber of a "basement" isovelocity halfspace. The general pressure field description consists of a modal sum and continuum as in:

$$
\mathrm{p}(\mathrm{r}, \mathrm{z})=\mathrm{j} \pi \sum_{\mathrm{n}=1}^{\infty} \mathrm{u}_{\mathrm{n}}^{*}\left(\mathrm{z}_{0}\right) \mathrm{u}_{\mathrm{n}}(\mathrm{z}) \mathrm{H}_{0}^{(1)}\left(\mathrm{k}_{\mathrm{n}} \mathrm{r}\right)+\mathrm{I}(\mathrm{r})
$$

Attenuation effects are incorporated by a small imaginary term in the horizontal wavenumber, $\mathrm{k}_{\mathrm{n}}$.

If the asymptotic form of the Hankel function is used (equation 2.1.10) and the attenuation term is included, the pressure field description becomes:

$$
\mathrm{p}(\mathrm{r}, \mathrm{z}) \approx \mathrm{j} \pi \sum_{\mathrm{q}=1}^{\mathrm{p}} \mathrm{a}_{\mathrm{q}} \mathrm{u}_{\mathrm{q}}^{*}\left(\mathrm{z}_{0}\right) \mathrm{u}_{\mathrm{q}}(\mathrm{z}) \sqrt{\frac{2}{\pi \mathrm{r}}} \mathrm{e}^{\mathrm{j}\left(\mathrm{kqr}-\frac{\pi}{4}\right)+\alpha \mathrm{q} r}
$$




\subsection{Prony's Method}

Prony's method is a parameter estimation technique in which the model parameters are varied to fit the observed data. Parameter estimation approaches involve the use of a priori knowledge in the intelligent selection of an appropriate model[29]. In Prony's method, the signal is considered to be composed of a linear combination of damped complex exponentials[30]. Prony's method is by no means the only model which may be applied to the ocean waveguide (for example Pisarenko or autoregressive moving average (ARMA) modelling might also be used)[31]. The use of Prony's algorithm was driven by two factors. First, the method has the advantage of requiring short data lengths ( a small range aperture) to yield high resolution wavenumber estimates. Second, the modal structure of far field propagation in the shallow water waveguide can fit the Prony model very well. These two characteristics lend credibility to the application of the technique to shallow water waveguide propagation.

In 1795, Gaspard Riche, Baron de Prony, proposed an interpolation scheme in which a deterministic model was assumed and the equally spaced data was used to fit this deterministic model[31]. The method consisted of an exact fit of the data points to exponentials; the evolution of the algorithm since then has been significant. The insight of the solution method and the ensuing three step process has endured although the expanded algorithms additionally address issues such as stability, robustness in noise and least square fits.

The model used by the algorithm is a weighted sum of complex damped exponentials. Consideration of the exactly determined case allows identification of the steps used in the algorithm; the development of the extended Prony method(least squares fit for an overdetermined system) is an enhancement of this basic procedure. 
The observed data, $y[n]$, is assumed to fit the model:

$$
y[n]=\sum_{q=0}^{p} \cdot A_{q} \exp \left[\left(\alpha_{q}+j k_{q}\right) n T+j \theta_{q}\right]
$$

with

$$
\begin{aligned}
& \mathrm{p}=\text { model order } \\
& \mathrm{T}=\text { sampling range } \\
& \mathrm{A}_{\mathrm{q}}=\text { amplitude } \\
& \alpha_{\mathrm{q}}=\text { damping factor } \\
& \mathrm{k}_{\mathrm{q}}=\text { wavenumber } \\
& \theta_{\mathrm{q}}=\text { initial phase }
\end{aligned}
$$

Regrouping terms:

$$
y[n]=\sum_{\mathrm{q}=0}^{\mathrm{p}-1} \mathrm{v}_{\mathrm{k}} \mathrm{z}_{\mathrm{q}}^{\mathrm{n}}
$$

where

$$
\begin{aligned}
& v_{q}=A_{q} \exp \left(j \theta_{q}\right) \\
& z_{q}=\exp \left[\left(\alpha_{q}+j k_{q}\right) T\right]
\end{aligned}
$$

For $\mathrm{p}$ data points, the system may be expressed in matrix form:

$$
\left(\begin{array}{cccc}
\mathrm{z}_{0}^{0} & \mathrm{z}_{1}^{0} & \ldots & \mathrm{z}_{\mathrm{p}-1}^{0} \\
\mathrm{z}_{0}^{1} & \mathrm{z}_{1}^{1} & \ldots & \mathrm{z}_{\mathrm{p}-1}^{1} \\
\vdots & \vdots & : & \vdots \\
\mathrm{z}_{0}^{\mathrm{p}-1} & \mathrm{z}_{1}^{\mathrm{p}-1} & \ldots & \mathrm{z}_{\mathrm{p}-1}^{\mathrm{p}-1}
\end{array}\right)\left(\begin{array}{c}
\mathrm{v}_{0} \\
\mathrm{v}_{1} \\
: \\
\mathrm{v}_{\mathrm{p}-1}
\end{array}\right)=\left(\begin{array}{c}
\mathrm{y}[0] \\
\mathrm{y}[1] \\
\vdots \\
y[\mathrm{p}-1]
\end{array}\right)
$$

or

$$
\mathrm{Z} \underline{\mathrm{V}}=\underline{\mathrm{Y}}
$$

In order to solve for the complex quantities $\mathrm{v}_{\mathrm{q}}$ and $\mathrm{z}_{\mathrm{q}}$, we will decouple equation (2.2.3) by solving for the $z_{q}$ 's. The insight offered by Prony in this scheme was that one approach 
for finding the $z_{q}$ 's is the solution of a homogeneous linear constant coefficient difference equation (LCCDE).

The first two steps of Prony's method solve for the $z_{q}$ 's as follows:

Consider the observed signal,y[n], to be the output of an all pole filter driven by an unit impulse function (Fig 2.2.1):

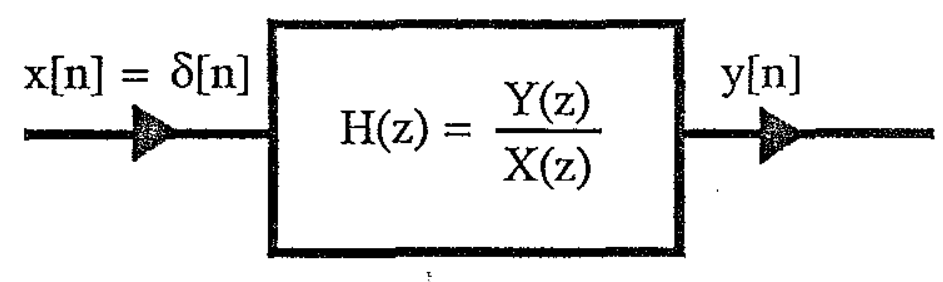

Fig 2.2.1 Filter Model of Observed Signal

For this model:

$$
\mathrm{H}(\mathrm{z})=\frac{1}{1+\sum_{\mathrm{q}=1}^{\mathrm{p}} \mathrm{a}_{\mathrm{q}} z^{-\mathrm{q}}}=\frac{1}{\prod_{\mathrm{r}=0}^{\mathrm{p}-1}\left(1-\mathrm{z}_{\mathrm{r}} \mathrm{z}^{-1}\right)}
$$

Since $\mathrm{H}(\mathrm{z})$ is defined as $\frac{\mathrm{Y}(\mathrm{z})}{\mathrm{X}(\mathrm{z})}$, cross multiplying equation (2.2.4) yields:

$$
\mathrm{Y}(\mathrm{z})\left[1+\sum_{\mathrm{q}=1}^{\mathrm{p}} \mathrm{a}_{\mathrm{q}} \mathrm{z}^{-\mathrm{q}}\right]=\mathrm{X}(\mathrm{z})
$$

Defining $\mathrm{a}_{0}=1$ and taking the inverse $\mathrm{Z}$ transform results in an LCCDE:

$$
x[n]=\sum_{q=0}^{p} a_{q} y[n-q]
$$

The homogeneous portion of the LCCDE is:

$$
\sum_{\mathrm{q}=0}^{p} \mathrm{a}_{\mathrm{q}} \mathrm{y}[\mathrm{n}-\mathrm{q}]=0
$$

Expanding by one term (recall a $0=1$ ):

$$
y[n]+\sum_{q=1}^{p} a_{q} y[n-q]=0
$$


This yields a set of equations over the observed data range $0 \leq \mathrm{n} \leq 2 \mathrm{p}-1$ :

$$
\left(\begin{array}{cccc}
y[p-1] & y[p-2] & \ldots & y[0] \\
y[p] & y[p-1] & \ldots & y[1] \\
: & : & : & : \\
y[2 p-2] & y[2 p-3] & \ldots & y[p-1]
\end{array}\right)\left(\begin{array}{c}
a_{1} \\
a_{2} \\
: \\
a_{p}
\end{array}\right)=-\left(\begin{array}{c}
y[p] \\
y[p+1] \\
\vdots \\
y[2 p-1]
\end{array}\right)
$$

The first step of Prony's method involves obtaining the coefficients $\mathrm{a}_{\mathrm{q}}$ 's by solving equation (2.2.9) using the $2 \mathrm{p}$ complex data points $\mathrm{y}[\mathrm{n}]$. Notice that the $\mathrm{a}_{\mathrm{q}}$ 's were defined in equation (2.2.4) as the coefficients of a polynomial which had the $z_{q}$ 's as the roots. Obtaining the roots of the equationis the second step of the process. Now that the $\mathrm{z}_{\mathrm{q}}$ 's are available, the third and final step of the process is to solve the exactly determined sytem defined by equation (2.2.3). The nonlinear aspect of the problem has been isolated in the second step. An attempt to solve equation (2.2.1) directly by an error minimization scheme results in a nonlinear set of equations which must be solved by Newton's method or some other iterative approach.

In the last section, we identified the three steps used to fit observed data to a model defined by an exactly determined set of equations. In practice, the amount of data typically exceeds the model order, which allows extension of the procedure to encompass a least squares estimation. The advantage of the least squares technique is that the issues of noise and stability may be approached using the rich theoretical material available in linear prediction, lattice, and autoregressive (AR) filter design[32]. This section will take a closer look at the three steps in Prony's method and identify the algorithm used in our work.

The first step contains the greatest variety of approaches. The identification of the "best" polynomial coefficients is complicated by the overdetermined situation; instead of solving equation (2.2.9) directly, an error criterion must be minimized. There are three 
general ways in which the error criterion is minimized, one "direct" and two "indirect". The use of "indirect" and "direct" qualifiers is an identification of how the coefficient vector is determined. In the "direct" method, operations take place directly on the signal matrix while the "indirect" procedures use the observed data to generate an exactly determined set of linear equations. The "indirect" methods are the techniques most frequently used and will be discussed first.

The "indirect" methods will be presented here from the viewpoint of linear prediction theory[29,32-34]. A simple way of expressing the linear prediction philosophy is that the $p+1$ output, ie $y[p+1]$, may be predicted by using a linear combination of the last p outputs, ie. $y[p], y[p-1], \ldots y[1]$. By using our all pole model in Figure 2.2.1, we can make use of its $\operatorname{LCCDE}$ (equation (2.2.6)) and replace the input, $x[n]$, by $\delta[n]$. Since $a_{0}=1, \mathrm{LCCDE}$ may be rewritten as:

$$
y[n]=-\sum_{q=1}^{p} a_{q} y[n-q]+\delta[n]
$$

Denoting $\vartheta$ as the predicted estimate of $\mathrm{y}[\mathrm{p}+1]$ :

$$
\vartheta=-\sum_{q=1}^{p} a_{q} y[n-q]
$$

The error,e[n], is now defined as the difference between the observed and predicted value:

$$
e[n]=y[n]-\vartheta
$$

The coefficients $a_{q}$ which minimize the energy in the error signal are found by the "normal equations". In matrix form, the quantity to be minimized is:

$$
\varepsilon=\underline{e}^{\mathrm{T}} \underline{\underline{e}}
$$

The error equations of equation (2.2.12) may be gathered in matrix form:

$$
\mathrm{X}-\mathrm{C} \underline{\mathrm{a}}=\underline{\mathrm{e}}
$$

Expanding (2.2.13) and minimizing yields the following relationship:

$$
\mathrm{C}^{\mathrm{H}} \mathrm{Ca}=\mathrm{C}^{\mathrm{H}_{\mathrm{Y}}}
$$


which are known as the "normal equations". These equations exploit the orthogonality of the error vector to the basis set formed by the columns of the signal matrix. The range of values over which the error is minimized differentiates the two "indirect" methods, which are known as the autocorrelation and autocovariance methods[33].

The autocorrelation method minimizes the error over an infinite duration signal. The method assumes a stationary process and yields a matrix system which has a Toeplitz structure. This symmetric, positive semidefinite characteristic allows the use of Levinson's recursions for an efficient solution algorithm. The main advantage of the autocorrelation method is that the ensuing filter is theoretically guaranteed to be stable (all of the poles within the unit circle of the $\mathrm{z}$ plane). Care must be taken in the numerical implementation of the technique to avoid accumulated roundoff errors from making the autocorrelation matrix ill conditioned.

The disadvantages stem from the minimization of the error over an infinite interval. In an all pole model, the impulse response will be infinite in duration. The finite amount of data available to the user is an implicit windowing of an infinite duration signal. This windowing effect changes the autocorrelation coefficients in the matrix, forcing an estimate (rather than determination) of the autocorrelation coefficients. The result of spreading the error over an infinite interval is that the model generated from all pole data will not match the actual system. Zero padding and application of windows to the available data minimize the effects of finite data length but these techniques may be hazardous in a high resolution spectrum analyzer situation.

The more common "indirect" approach in use today is the autocovariance method[3,35,36]. The error is minimized over the finite length of data. In stochastic theory this equates to the nonstationary case modelled as locally stationary. The matrix system which is solved for the $\mathrm{a}_{\mathrm{q}}$ 's is positive semi-definite but not Toeplitz. Although Levinson's recursions cannot be used to solve the system, there are algorithms, most 
notably Marple's, which address efficient solution techniques for this method. By minimizing the error over a finite interval, the autocovariance method will match all pole data to the generating system. As the data length increases, the covariance method will approach the autocorrelation technique.

A drawback to the covariance method is the lack of a stability theorem to guarantee the filter coefficients will describe a stable filter. This drawback was addressed by Burg, who developed an algorithm which constrained the problem to yield a stable filter[37,38]. Specifically, this is done by changing the error minimization problem. In addition to the error defined above, called the forward error, a new error, called the backwards error, is specified. Burg's rationale was that the stationary signal should "look" the same going forward and backwards through the data set. The error criteria to be minimized in a Burg algorithm is the sum of the forward and backward errors. Although summing the error over twice as many points is advantageous in a short data set, blind application of Burg's algorithm (also known as the modified covariance method) is dangerous. Specifically, the problem lies in the assumption of the signal appearing the same regardless of the direction of data set traversal. A sinusoid does indeed have this characteristic but the presence of damping (placing an exponential decaying envelope on the sinusoid) requires careful examination of the physical system before applying the Burg forward-backward error sum[2]. In the tradeoff between damping and stability, the decision is usually made to assume an undamped system in return for the assured stability. In the shallow water waveguide, constraining the pressure field to consist of undamped sinusoids is reasonable for propagating modes but not for the leaky or virtual modes which may be represented as damped sinusoids. In addition, although the assumption that the wavenumbers are real (no damping) is acceptable since the propagating modes have small damping factors, the user must then have other methods available to estimate mode attenuation. 
The approach explored in this thesis is the "direct" method in which the least squares operation is performed directly on the signal matrix. The technique is hampered by the lack of a stability theorem but this was not found to be a problem in practice. In the "direct" approach, a QR decomposition is used. The QR decomposition is a very stable, fast technique which uses Householder (orthogonal) matrices to orthogonalize the original matrix $[39,40]$. The use of Householder matrices results in a decomposition of the A matrix into

$$
A_{p \times q}=Q_{p \times p} R_{p \times q}
$$

in which the first q columns of $\mathbf{Q}$ form an orthonormal basis for the column space of $\mathbf{A}$ and the last $p-q$ columns form a basis for the left nullspace of $\mathbf{A}$ (ie, the last $p-q$ columns are perpendicular to the first $q$ columns). The first $\mathbf{n}$ rows of $\mathbf{R}$ form an upper triangular matrix; the columns of $\mathbf{R}$ are formed by successive Householder matrices operating on corresponding columns of $\mathbf{A}$.

The least squares problem may be solved by considering:

$$
A \underline{x}=\underline{b}
$$

with

$$
\begin{aligned}
& \mathbf{A}=p \times q \text { matrix }(p>q \text { since overdetermined) } \\
& \underline{x}=q \times 1 \text { vector (in Prony's method, these are the } a_{q} \text { 's) } \\
& \underline{b}=p \times 1 \text { vector. }
\end{aligned}
$$

Next, the normal equations for the complex matrix $\mathbf{A}$ are expressed as:

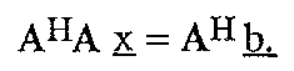

Substituting QR for A in this system yields the following:

$$
\begin{aligned}
& \left(\mathrm{R}^{\mathrm{H}} \mathrm{Q}^{\mathrm{H}}\right) \mathbf{Q R} \underline{\mathrm{x}}=\mathrm{R}^{\mathrm{H}} \mathrm{Q}^{\mathrm{H}} \underline{\mathrm{b}} \\
& \left(\mathrm{Q}^{\mathrm{H}} \mathrm{Q}\right) \mathrm{R} \underline{\mathrm{x}}=\mathrm{Q}^{\mathrm{H}} \underline{\mathrm{b}} \\
& \mathrm{R}_{\underline{\mathrm{x}}}=\mathrm{Q}^{\mathrm{H}} \underline{\mathrm{b}} .
\end{aligned}
$$

So, 


$$
\underline{x}=R^{-1} Q^{H} \underline{b}
$$

The advantage to using this approach over the normal equations is evident when the columns of $\mathbf{A}$ are barely uncorrelated. The ensuing calculation of $\mathbf{A}^{\mathrm{H}} \mathbf{A}$ will amplify round off etrors due to this matrix being ill conditioned. The orthogonal matrix decomposition approach avoids this problem and the round off error accumulations are at a minimum[41].

Specification of a model order is inherent in the first step. The determination of a "good" order is complicated by using an all pole (also known as an autoregressinve [AR]) model to represent a pole zero (also knowns as ARMA) process. Even if the number of poles are known apriori, use of the exact number of system poles may not yield a good result. If the model order is underdetermined, the "spectrum" will be smooth and smeared. If the order is overdetermined, the model is likely to have spurious peaks[32]. The empirical rule used by the signal processing community of overestimating the model order is supported by two assumptions. First, underestimation of model order will not identify true poles while overestimation will tend to identify these poles. Although the model is forced to find parameters to fit the specified system, the energy of the arbitrary poles (ie, those in excess of actual system order) is quite small. Second, the presence of noise in the data may be modelled as zeros[31]. An ARMA process represented by an AR model requires overspecification of model order (actually, the bias of the estimation decreases as the order increases).

Various researchers have suggested analytical methods to estimate model order[33,42-44]. Akaike[42] has suggested a final prediction error method and a cost minimization method in which a cost is assigned for extra coefficients which do not reduce model order. Criticism of the final prediction method is that it yields too low a model order while the cost minimization method is said to have statistical inconsistencies. To date, there is no common approach for identifying a good model order. In the algorithm used here, the model order specification is left to the user. A first approach used an singular value 
decomposition(SVD) of the signal matrix to obtain singular values of the system. A sudden decrease in the magnitude of the singular values was the breakpoint of estimating the actual modes of the system. Two problems with this approach are that the "breakpoint" is not clearly defined as noise is introduced and the optimum model order is still not specified by examining the singular values. A more subjective approach made possible by the interactive nature of the algorithm was to increase the order and examine the outputs. When the number and values of the wavenumbers of propagating modes (identified by low damping and high amplitude) stopped changing as order increased, the model was said to have sufficient order.

Polynomial rooting is the second step of the Prony process. Since this is done numerically, a robust algorithm must be used. The nonlinear aspect of the parameter fitting is located in this portion of the algorithm; slight errors in the coefficients may result in significant changes in the roots[41]. For example, the polynomial $x^{4}-10 x^{3}+35 x^{2}-50 x$ +24 has roots of $(x-1)(x-2)(x-3)(x-4)$. A change of $0.5 \%$ in the second coefficient yields a polynomial of $x^{4}-10.05 x^{3}+35 x^{2}-50 x+24$, which has roots of $(x-0.992)(x-$ $2.340-j 0.2269)(x-2.340+j 0.2269)(x-4.378)$. The choice of a complex rooting routine by Jenkins and Traub and double precision calculations are the tools used to reduce errors in this section of the algorithm[45]. While the three stage rooting algorithm has performed well, the sensitivity of the roots of a polynomial to the coefficient values indicates the choice of algorithms in step one may be the major contributing factor to the accuracy of the final model.

The third and final step of the routine is the solution of the overdetermined version of the linear system expressed in equation (2.2.3). The minimization of least square error technique is used; the result is the normal equations approach outlined in equation (2.2.15). $A Q R$ decomposition or the usual least squares technique $\left(x=\left(A^{H} A\right)^{-1} A^{H} \underline{b}\right)$ may be used[46]. The algorithm exercised in the thesis research used the second method and 
incorporated a Cholesky decomposition scheme to exploit the Hermitian symmetry of $\mathrm{A}^{\mathrm{H}} \mathrm{A}$. 


\subsection{Application of Prony's Method to the Shallow Water Waveguide}

In the section on underwater acoustic theory, an expression for the far field acoustic pressure as a sum of normal modes was developed. While the algorithm for using Prony's method to fit parameters to a deterministic model was outlined in the last section, the task of transforming the pressure field to a suitable form remains. We will now develop a model of the shallow water waveguide in a format which fits that assumed in the last section.

From equation (2.1.8), the pressure field may be expressed as[13]:

$$
\mathrm{p}(\mathrm{r}, \mathrm{z})=\mathrm{j} \pi \sum_{\mathrm{q}=1}^{\mathrm{p}} \mathrm{a}_{\mathrm{q}} \mathrm{u}_{\mathrm{q}}^{*}\left(\mathrm{z}_{0}\right) \mathrm{u}_{\mathrm{q}}(\mathrm{z}) \mathrm{H}_{0}^{(1)}\left(\mathrm{k}_{\mathrm{q}} \mathrm{r}\right)+\mathrm{I}(\mathrm{r})
$$

The far field contribution of the continuum, I(r), may be neglected and the asymptotic approximation for the Hankel function of

$$
\mathrm{H}_{0}^{(1)}\left(\mathrm{k}_{\mathrm{q}} \mathrm{r}\right) \approx \sqrt{\frac{2}{\pi \mathrm{r}}} \mathrm{e}^{\mathrm{j}\left(\mathrm{kqr}-\frac{\pi}{4}\right)}
$$

is substituted into equation (2.3.1) to yield:

$$
\mathrm{p}(\mathrm{r}, \mathrm{z}) \approx \mathrm{j} \pi \sum_{\mathrm{q}=1}^{\mathrm{p}} \mathrm{a}_{\mathrm{q}} \mathrm{u}_{\mathrm{q}}^{*}\left(\mathrm{z}_{0}\right) \mathrm{u}_{\mathrm{q}}(\mathrm{z}) \sqrt{\frac{2}{\pi \mathrm{r}}} \mathrm{e}^{\mathrm{j}\left(\mathrm{kqr}-\frac{\pi}{4}\right)+\alpha \mathrm{q} r}
$$

Since the pressure field is measured on a horizontal array, $p(r, z)$ will be expressed as $p(r)$ and the depth dependence will be incorporated into a constant, $\mathrm{A}_{\mathrm{q}}$, in the following manner:

$$
p(r) \approx \sum_{q=1}^{p} \frac{A_{q}}{\sqrt{r}} \exp [(\alpha q+j k q) r+j \theta q]
$$

The data available for processing are actually samples of the pressure field rather than the continuous pressure field itself. The discrete samples allow $\mathrm{r}$ in equation (2.3.4) to be replaced by $\mathrm{nT}$ in which $\mathrm{T}$ is the sampling range. This assumes equally spaced data 
points; in practice, a cubic spline interpolation scheme is used to ensure proper spacing of the input data.to the algorithm. Experimental results indicate the sampling must be dense enough to meet the Nyquist criteria to avoid aliasing.[25].

The last step in modeling the pressure field in a Prony format is the elimination of the $\frac{1}{\sqrt{\mathrm{r}}}$ term in equation (2.3.4). The data is multiplied by the $\sqrt{\mathrm{r}}$ to yield the model:

$$
y[n]=p(r) \sqrt{r}=\sum_{q=0}^{p-1} A_{q} \exp [(\alpha q+j k q) n T+j \theta q]
$$

where

$$
\begin{aligned}
& A_{\mathrm{q}}=\operatorname{lj\pi } \mathrm{a}_{\mathrm{q}} \mathrm{u}_{\mathrm{q}}^{*}\left(\mathrm{z}_{0}\right) \mathrm{u}_{\mathrm{q}}(\mathrm{z}) \mid=\text { amplitude } \\
& \alpha \mathrm{q}=\operatorname{Im}\left(\mathrm{k}_{\mathrm{q}}\right)=\text { damping } \\
& \mathrm{kq}=\operatorname{Re}\left(\mathrm{k}_{\mathrm{q}}\right)=\text { modal eigenvalues } \\
& \theta \mathrm{q}=\frac{\pi}{4}+\angle\left[j \pi \mathrm{a}_{\mathrm{q}} \mathrm{u}_{\mathrm{q}}^{*}\left(\mathrm{z}_{0}\right) \mathrm{u}_{\mathrm{q}}(\mathrm{z})\right]=\text { initial phase }
\end{aligned}
$$

This model may be further compacted in the form of equation (2.2.2)

$$
\mathrm{y}[\mathrm{n}]=\sum_{\mathrm{q}=0}^{\mathrm{p}-1} \mathrm{v}_{\mathrm{q}} \mathrm{z}_{\mathrm{q}}^{\mathrm{n}}
$$

where

$$
\begin{aligned}
& \mathrm{v}_{\mathrm{q}}=\mathrm{A}_{\mathrm{q}} \exp \left(j \theta_{\mathrm{q}}\right) \\
& \mathrm{z}_{\mathrm{q}}=\exp \left[\left(\alpha_{\mathrm{q}}+j \mathrm{k}_{\mathrm{q}}\right) \mathrm{T}\right]
\end{aligned}
$$

Once the $\mathrm{v}_{\mathrm{q}}$ and $\mathrm{z}_{\mathrm{q}}$ 's are obtained through Prony's method, the parameters of the model are generated via:

$$
\begin{aligned}
& \mathrm{k}_{\mathrm{q}}=\frac{\tan ^{-1}\left(\frac{\operatorname{m}\left(\mathrm{z}_{\mathrm{q}}\right)}{\operatorname{Re}\left(\mathrm{z}_{\mathrm{q}}\right)}\right)}{\mathrm{T}} \\
& \alpha_{\mathrm{q}}=\frac{\ln \left|\mathrm{z}_{\mathrm{q}}\right|}{\mathrm{T}} \\
& \mathrm{A}_{\mathrm{q}}=\left|\mathrm{v}_{\mathrm{q}}\right|
\end{aligned}
$$




$$
\theta q=\tan ^{-1}\left(\frac{\operatorname{Im}\left(v_{q}\right)}{\operatorname{Re}\left(v_{q}\right)}\right)
$$

An examination of the output of the program for a simple model is useful as a guide to gaining physical insight into the analysis results. The algorithm described in section 2.1 was incorporated in a FORTRAN program called PRAWNS (PRony Analysis of Waveguide for Nominal Spectrum). A sound speed profile (summarized in figure 2.3.1) was developed and the corresponding complex pressure field versus range data was generated by SNAP[47]. The sound speed profile is a simplified version of the experimentally determined profile of the water column and sediment layers off Nantucket Island in Massachusetts. SNAP, a normal mode acoustic propagation modelling program, generates a far field approximation of a pressure field from a user defined profile and frequency (in this case, $220 \mathrm{~Hz}$ ). In addition to a pressure vs. range output, SNAP provides a list of the modal eigenvalues (the wavenumbers), the normalized eigenfunctions (the $\mathrm{u}_{\mathrm{q}}(\mathrm{z})$ 's) and the attenuation coefficients. This "ground truth" permits a reasonable method of examining the PRAWNS output.

Table 2.3.1 provides the SNAP attenuation and wavenumber outputs for this profile. Table 2.3.2 is a partial PRAWNS output of two different range intervals. (The specific relationship among the model specifications listed in table 2.3.2 is covered in Chapter 3). A brief examination of the PRAWNS outputs in table 2.3.2 will highlight some program characteristics; the information provided by this simple analysis is rich. First, the PRAWNS analysis provides a means of identification of the propagating modes in the overspecified Prony model. As expected, the propagating modes are marked by high amplitude and low damping. As the model order is increased, the damping and amplitude remained constant for a given range block. When the order is vastly overspecified, there is a small change in the propagating mode amplitudes as the energy is 


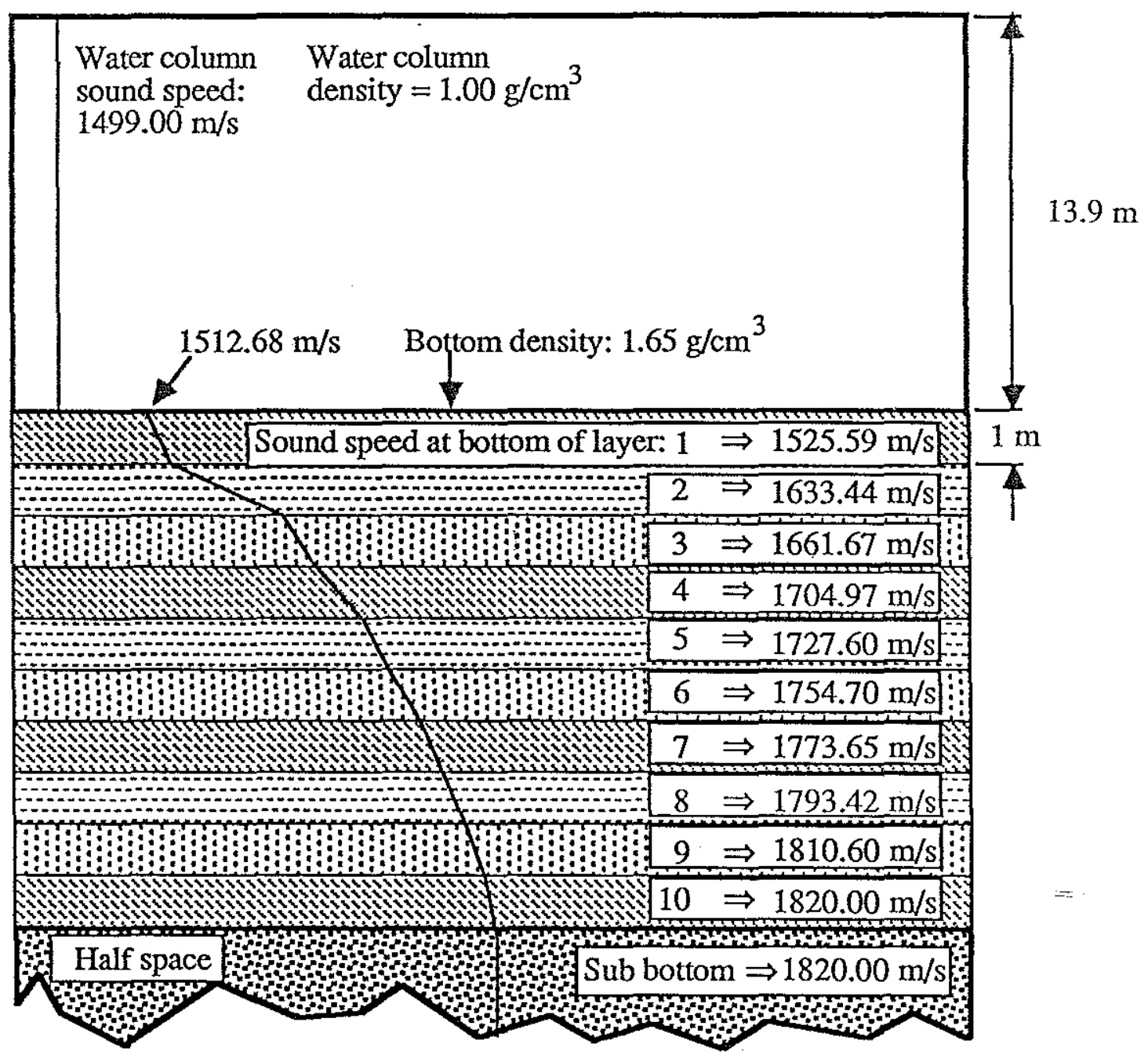

Fig 2.3.1 Nantucket Profile Used in SNAP Program 
forced to be distributed among the user specified order. In viewing the tabular data, high amplitude and low damping proved to be a good indicator as to the actual order of the system (as mentioned in section 2.2.1, an analytic approach to obtain actual system order may be performed by an SVD of the signal matrix). Second, as mentioned in the introduction, the primary objective of the analysis is to obtain accurate wavenumber data. Comparison of the first three indices of table 2.3.2 with the modes of table 2.3.1 show a wavenumber match to five significant digits. This is most satisfying given that the aperture size is 90 meters ( 30 points of data used). Third, the damping factors output by PRAWNS are quite reasonable given the small aperture. It is expected that the small decay in energy of a propagating mode over the 90 meter range aperture would lead to difficulties in accurate estimation of the attenuation factor. There are two ways to attempt to improve this accuracy. The first method is to increase the range aperture and allow the energy of a given mode a greater distance to decay. The second method uses the amplitudes of a given mode as outlined in figure 2.3.2. The origin is set to some reference range and the PRAWNS algorithm is used on the range interval $R$ to $\mathrm{R}^{\prime}$. Since attenuation will cause an exponential decay of the amplitude of a given mode with respect to range, the amplitude of a particular mode, $A\left(\mathrm{r}_{0}\right)$, estimated by PRAWNS at a local reference range, $\mathrm{r}_{0}$, is given by:

$$
\mathrm{A}\left(\mathrm{r}_{0}\right)=\mathrm{A}(0) \exp \left[\alpha \mathrm{r}_{0}\right]=\mathrm{A}(0) \exp [\alpha \mathrm{R}] \exp [\alpha \delta \mathrm{r}]
$$

where

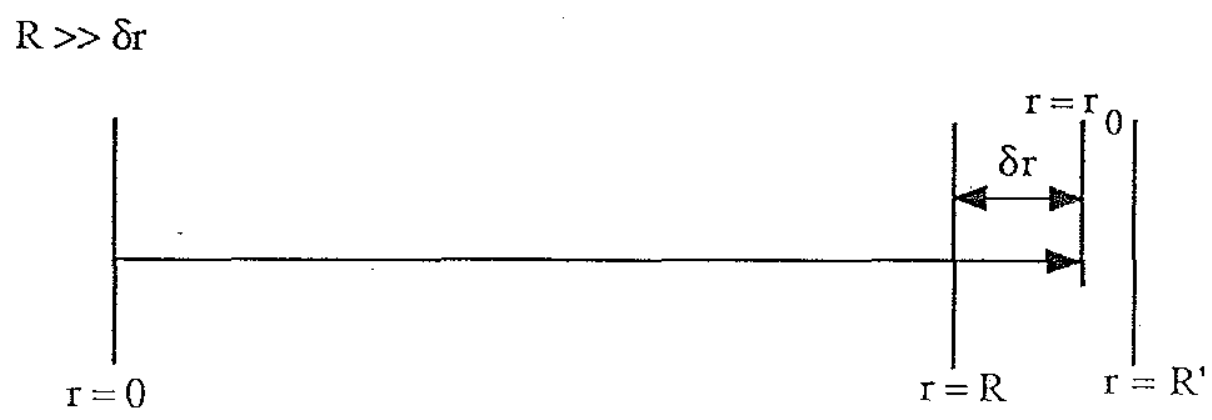

Fig 2.3.2 Amplitude decay vs range 
While the PRAWNS aperture may be too small to yield accurate attenuation estimates, the use of amplitude estimates from seperate range blocks may permit a better attenuation value. For a numerical example, consider the amplitude of mode 1 in the $1002-1149 \mathrm{~m}$ and the $1350-1497 \mathrm{~m}$ range blocks. Using a range difference of $1350-1002=348 \mathrm{~m}=$ $\Delta \mathrm{r}$, set up a simple ratio which takes into account the effect of attenuation in the model:

$$
\frac{A_{12}}{A_{11}}=e^{\alpha \Delta r} \text { where } A_{p q}=\text { mode } p \text { amplitude in range block } q \text {. }
$$

Substituting,

$$
\alpha=\frac{1}{348} \ln \left(\frac{0.1044188}{0.1076169}\right)=-8.67 \mathrm{E}-05
$$

Comparing the results in equation (2.3.7) to mode 1 of Table 2.3.1, we see this method is not a good choice in this trial. This may be due to poor amplitude estimates or a short range interval. 
Table 2.3.1 SNAP Output for Nantucket Profile

\begin{tabular}{|ccc|}
\hline Mode No. & Wavenumber (m-1) & Attenuation \\
1 & 0.9074474 & $7.995 \mathrm{E}-05$ \\
2 & 0.8546543 & $2.422 \mathrm{E}-04$ \\
3 & 0.7758756 & $1.067 \mathrm{E}-03$ \\
\hline
\end{tabular}

Table 2.3.2 PRAWNS Output for Nantucket Profile

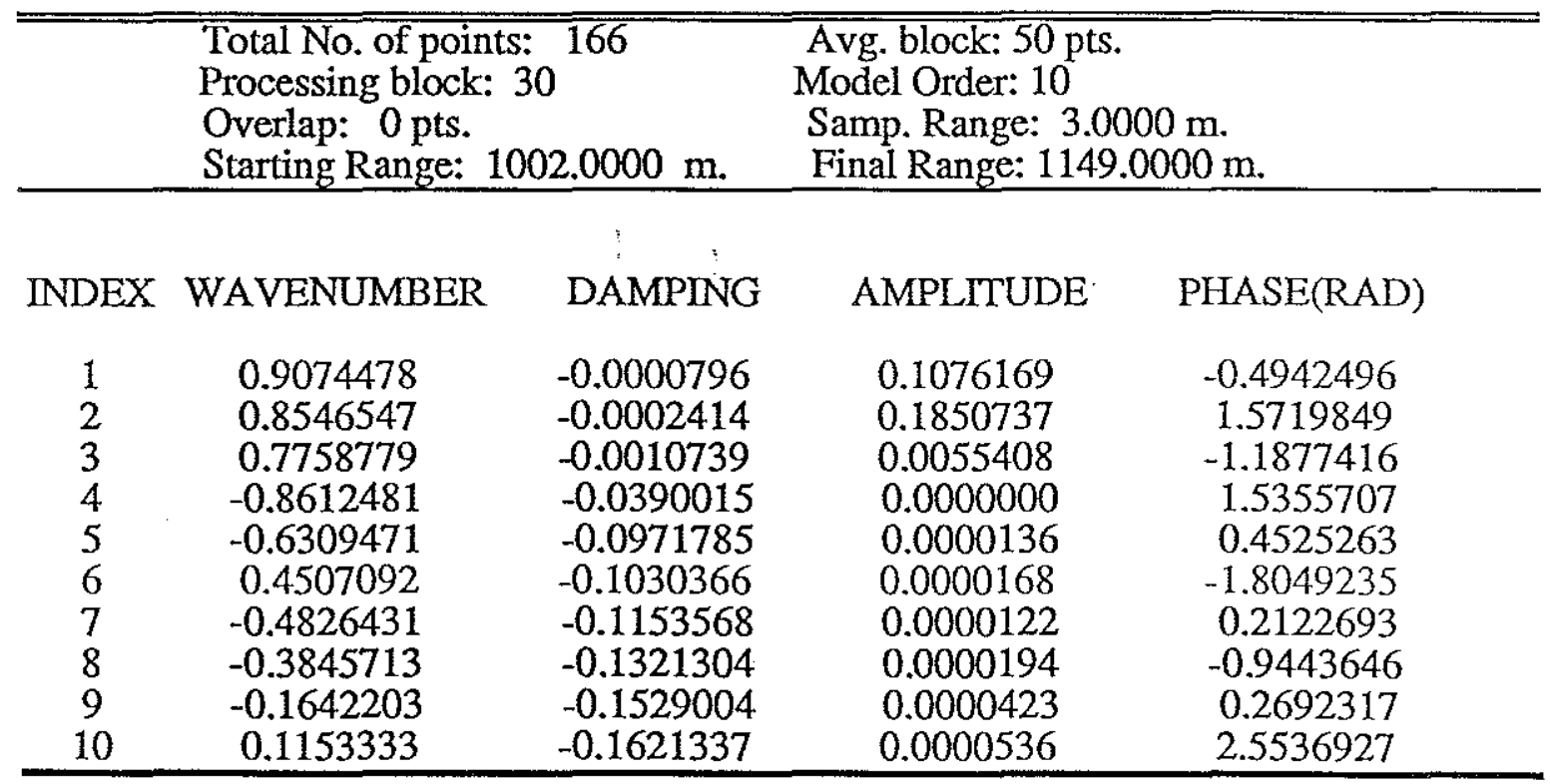

Total No. of points: 166

Processing block: 30

Overlap: 0 pts.

Starting Range: $1350.0000 \mathrm{~m}$.
Avg. block: 50 pts.

Model Order: 10

Samp. Range: $3.0000 \mathrm{~m}$.

Final Range: $1497.0000 \mathrm{~m}$.

\begin{tabular}{ccccc} 
INDEX & WAVENUMBER & DAMPING & AMPLITUDE & PHASE(RAD) \\
1 & 0.9074471 & -0.0000800 & 0.1044188 & -3.0543090 \\
2 & 0.8546540 & -0.0002421 & 0.1688871 & -2.0942594 \\
3 & 0.7758755 & -0.0010671 & 0.0036974 & -0.0749611 \\
4 & 0.2020056 & -0.0030457 & 0.0000000 & -2.3543923 \\
5 & 0.3843271 & -0.0272609 & 0.0000000 & 0.6840160 \\
6 & -0.5876253 & -0.0380173 & 0.0000000 & 2.2279577 \\
7 & -0.8681644 & -0.0567308 & 0.0000000 & -0.6433010 \\
8 & -0.0685432 & -0.0614808 & 0.0000000 & 0.5810484 \\
9 & -0.3724043 & -0.0977833 & 0.0000001 & 0.9746809 \\
10 & -0.4323751 & -0.1299475 & 0.0000001 & -2.0567591 \\
\hline
\end{tabular}


As the range between the analysis blocks increases, the decay in energy will be greater and the attenuation estimate should improve. This alternate method of estimating attenuation is also prone to noise effects manifested in amplitude estimations.

Because of the isovelocity water column of the Nantucket profile of figure 2.3.2, the mode shapes in the water column are not distorted by gradients. The mode shapes provided as part of the SNAP output may be used as a comparison to the Prony analysis for a vertical array. This was synthesized by using the sound speed profile of figure 2.3.1 but changing the receiver depth. The receiver depth was changed in half meter increments from 0.5 to 13.5 meters. A range aperture of 375 meters was specified and the PRAWNS program was used to analyze the modal structure at 500, 1000 and 1400 meters (see figure 2.3.3).

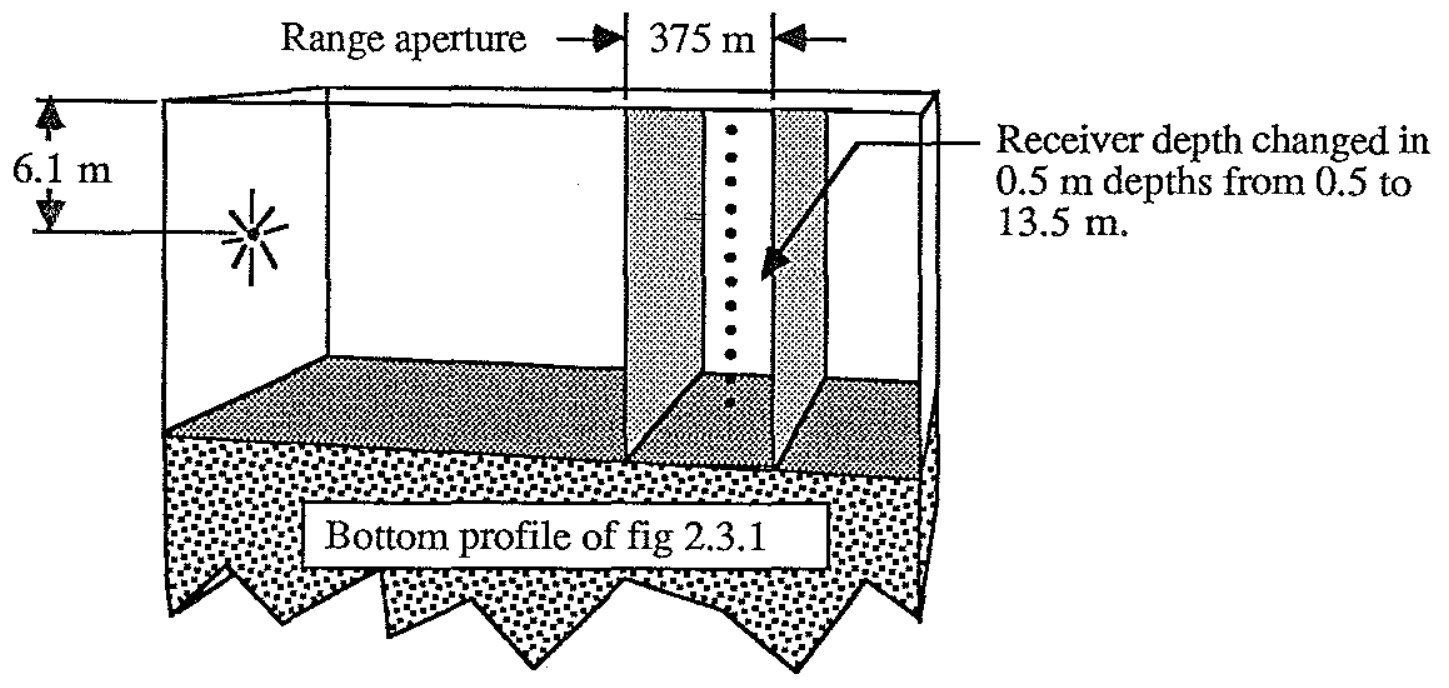

Fig 2.3.3 Vertical Array of Nantucket Profile 
SNAP and PRAWNS mode shapes $(500 \mathrm{~m})$

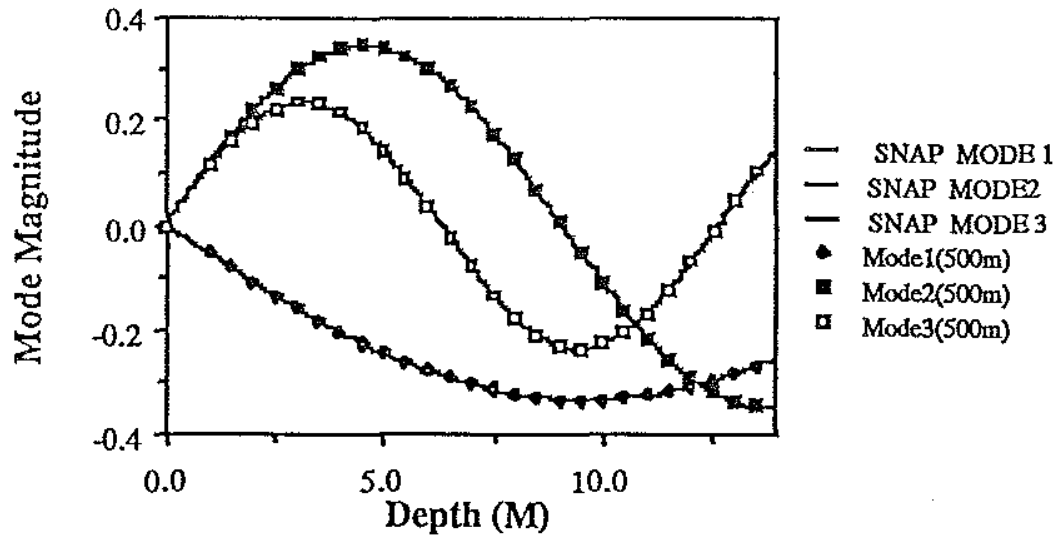

SNAP and PRAWNS mode shapes $(1000 \mathrm{~m})$

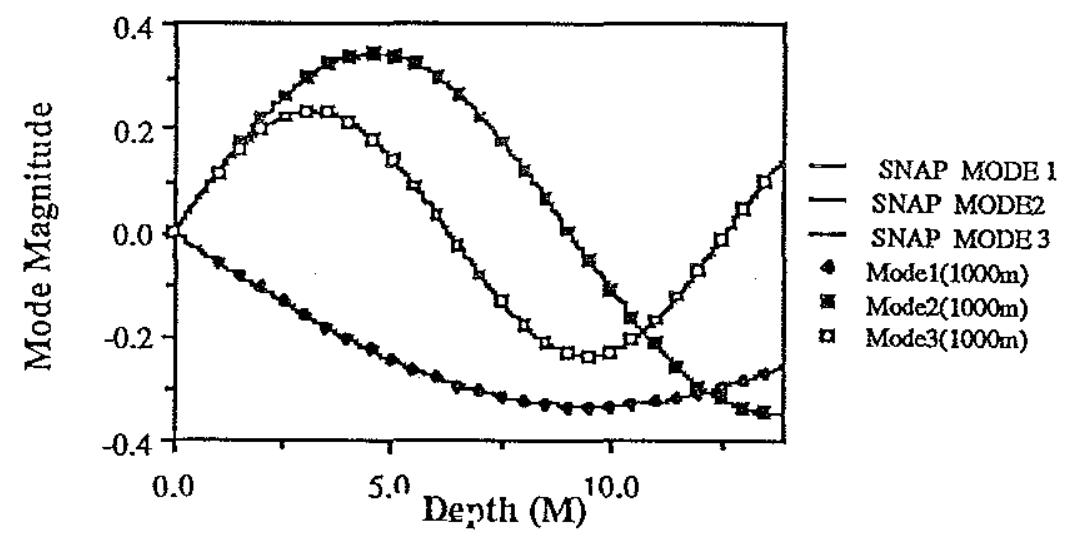

SNAP and PRAWNS mode shapes (1400 m)

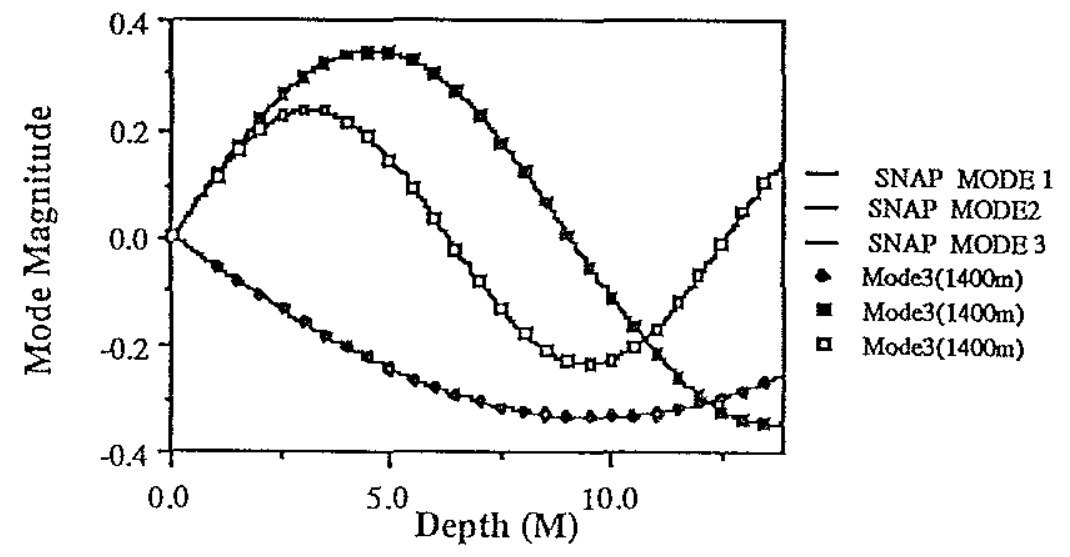

Figure 2.3.4 Vertical Mode Shapes for PRAWNS and SNAP 
The resulting amplitudes for each mode were scaled to match the normalized SNAP mode shapes at a particular depth (10 meters). The resulting close agreement is evident in figure 2.3.4. The larger aperture presents another opportunity to try the attenuation estimation method in equation (2.3.6). With a range interval of 249 meters and receiver depth of 10 meters, the results are summarized in table 2.3.3:

Table 2.3.3 Attenuation Estimation using Nantucket Profile

\begin{tabular}{|cccc|}
\hline Mode No. & Amplitude 1 & Amplitude 2 & Attenuation \\
& & & \\
2 & 0.2270556 & 0.2225807 & $-7.994 \mathrm{E}-05$ \\
3 & 0.0687738 & 0.0647491 & $-2.422 \mathrm{E}-04$ \\
& 0.0054065 & 0.0041446 & $-1.068 \mathrm{E}-03$ \\
\hline
\end{tabular}

The results are much closer to the SNAP results in Table 2.3.1. 


\subsection{Summary}

The cursory review of normal mode theory of section 2.1 was meant to emphasize key aspects of the shallow water waveguide problem. A general form for the pressure field was developed in the form of a modal sum and a continuum. A hard bottom example was used to demonstrate the form of the pressure field, the asymptotic form of the Hankel function and the effect of source receiver geometry. The Pekeris example demonstrated the discrete spectrum and continuous spectrum regions of more complex bottom models. The distinction between the discrete and continuous spectrum may be set by the wavenumber of a "basement" isovelocity halfspace. Attenuation effects were incorporated by a small imaginary term in the horizontal wavenumber, $\mathrm{k}_{\mathrm{n}}$.

In section 2.2, the basic three steps of Prony's method were developed. Upon closer examination of these steps, some of the options and rationale for choosing them were explained. The algorithm used in the remainder of the paper was presented; the particular method chosen for further investigation is different from the forms commonly found in the signal processing literature.

Section 2.3 tailored the modal sum representation of the pressure field into a form suitable for processing via Prony's method. Two applications of the method were presented. The first was a straightforward horizontal array application with a range aperture of 90 meters on data generated synthetically by SNAP. This application demonstrated the good agreement of wavenumbers between PRAWNS and SNAP; in addition, the distinguishing characteristics of propagating modes were presented. The second application simulated a vertical array by analysis of the same range blocks at different receiver depths. This example demonstrated use of the PRAWNS mode amplitudes to obtain vertical eigenfunction shapes and an alternate method for obtaining mode attenuation. 


\section{Chapter 3 \\ Prony's Method Evaluation}

\subsection{Depth Dependent Green's Function}

In the previous chapter, the pressure field was represented by a modal sum and continuum contribution. While the modal nature of sound propagation may be observed in the pressure field as an interference pattern, this is not the optimum domain for modal analysis. As the number of modes increases, the interference pattern becomes increasingly complex. An alternate description of the modal information may be obtained through a Hankel transform of the pressure field[48-50]. The result of this transformation is known as a depth dependent Green's function. This section outlines the form of the depth dependent Green's function.

A zero order Hankel transform is defined as[21]:

$$
\mathrm{H}(\mathrm{F}(\mathrm{r})\}=\mathrm{f}\left(\mathrm{k}_{\mathrm{r}}\right)=\int_{0}^{\infty} \mathrm{F}(\mathrm{r}) J_{0}\left(\mathrm{k}_{\mathrm{r}} \mathrm{r}\right) \mathrm{r} \mathrm{dr}
$$

With an inverse of:

$$
H\left\{f\left(k_{r}\right)\right\}=F(r)=\int_{0}^{\infty} f\left(k_{r}\right) J_{0}\left(k_{r} r\right) k_{r} d k_{r}
$$

That is, the Hankel transform is its own inverse. From equation (2.16), recall,

$$
\frac{1}{r} \frac{\partial}{\partial r}\left(r \frac{\partial p}{\partial r}\right)+\frac{\partial^{2} p}{\partial z^{2}}+k^{2}(z) p=-2 \frac{\delta(r)}{r} \delta\left(z-z_{0}\right)
$$

By taking the Hankel transform of both sides, the equation yields an ordinary differential equation:

$$
\left[\frac{d^{2}}{d z^{2}}+k^{2}(z)-k_{r}^{2}\right] g\left(k_{r}, z, z_{0}\right)=-2 \delta\left(z-z_{0}\right)
$$

In obtaining this equation, the following relationship is used:

$$
\mathrm{H}\left\{\frac{1}{\mathrm{r}} \frac{\mathrm{d}}{\mathrm{dr}}\left(\mathrm{r} \frac{\mathrm{d}}{\mathrm{dr}} \mathrm{F}(\mathrm{r})\right)\right\}=-\mathrm{k}_{\mathrm{r}}^{2} \mathrm{f}\left(\mathrm{k}_{\mathrm{r}}\right)
$$


The solution to equation (3.1.4), $\mathrm{g}\left(\mathrm{k}_{\mathrm{r}}, \mathrm{z}, \mathrm{z}_{0}\right)$, is known the depth dependent Green's function. An analytical solution to the equation may be found by application of Sturm Liouville theory. For a waveguide with the surface at $\mathrm{z}=0$ and bottom at $\mathrm{z}=\mathrm{h}$, the impedance boundary conditions may be expressed in the form:

$$
\begin{aligned}
& A_{1} \phi^{\prime}(0)+B_{1} \phi(0)=0 \\
& A_{2} \phi^{\prime}(h)+B_{2} \phi(h)=0
\end{aligned}
$$

Defining the Wronskian, $\mathrm{W}$, as $\mathrm{W}=\phi_{\mathrm{S}} \phi_{\mathrm{b}}{ }^{\prime}-\phi_{\mathrm{b}} \phi_{\mathrm{s}^{\prime}}$, permits the solution to equation (3.1.4) to be expressed as:

$$
\begin{array}{ll}
\mathrm{g}=\left\{\frac{-2}{\mathrm{~W}\left(\mathrm{z}_{0}\right)}\right\} \phi_{\mathrm{S}}\left(\mathrm{k}_{\mathrm{r}}, \mathrm{z}\right) \phi_{\mathrm{b}}\left(\mathrm{k}_{\mathrm{r}}, \mathrm{z}_{0}\right) & 0 \leq \mathrm{z} \leq \mathrm{z}_{0} \\
\mathrm{~g}=\left\{\frac{-2}{\mathrm{~W}\left(\mathrm{z}_{0}\right)}\right\} \phi_{\mathrm{S}}\left(\mathrm{k}_{\mathrm{r}}, \mathrm{z}_{0}\right) \phi_{\mathrm{b}}\left(\mathrm{k}_{\mathrm{r}}, \mathrm{z}\right) & \mathrm{z}_{0} \leq \mathrm{z} \leq \mathrm{h}
\end{array}
$$

The determination of the Green's function now requires identifying $\phi_{b}$ and $\phi_{\mathrm{S}}$, which are two solutions to a Sturm Liouville problem. Both solutions satisfy the homogeneous equation (3.1.4) and $\phi_{\mathrm{b}}$ satisfies the bottom boundary condition while $\phi_{\mathrm{S}}$ satisfies the surface boundary condition. In terms of reflection coefficients, for an isovelocity waveguide, the solutions at the surface, $\phi_{\mathrm{S}}$, and bottom, $\phi_{\mathrm{b}}$, the superposition of up and downgoing plane waves are[9,26]:

$$
\begin{aligned}
& \phi_{\mathrm{s}}=\mathrm{A}\left[\mathrm{e}^{-j \gamma z}+\mathrm{R}_{\mathrm{s}}\left(\mathrm{k}_{\mathrm{r}}\right) \mathrm{e}^{\mathrm{j} \gamma \mathrm{z}}\right] \text { and } \\
& \phi_{\mathrm{b}}=\mathrm{B}\left[\mathrm{ej}^{j \gamma z}+\mathrm{R}_{\mathrm{b}}\left(\mathrm{k}_{\mathrm{r}}\right) \mathrm{e}^{j \gamma(2 \mathrm{~h}-\mathrm{z})}\right]
\end{aligned}
$$

Substitution of these into the equation for the Green's function, equation (3.1.4), yields:

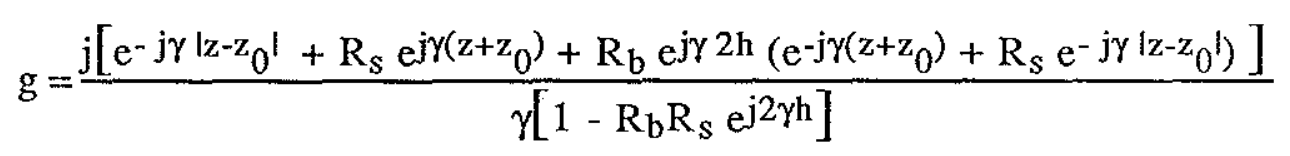

In the simple waveguide examples of chapter 2 , the surface reflection coefficient is $R_{S}=-1$; ie, the upper boundary is treated as a pressure release. The expression above holds equally well in multilayer waveguides in which the upper layer boundary condition is not constrained to be a 
pressure release mechanism. By the definition of the Hankel transform pair given above, the relationship between the depth dependent Green's function and the pressure field is then[14]:

$$
\begin{aligned}
& \mathrm{p}\left(\mathrm{r}, \mathrm{z}_{\mathrm{z}} \mathrm{z}_{0}\right)=\int_{0}^{\infty} \mathrm{g}\left(\mathrm{k}_{\mathrm{r}}, \mathrm{z}, \mathrm{z}_{0}\right) \mathrm{J}_{0}\left(\mathrm{k}_{\mathrm{r}} \mathrm{r}\right) \mathrm{k}_{\mathrm{r}} d \mathrm{k}_{\mathrm{r}} \quad \text { and } \\
& \mathrm{g}\left(\mathrm{k}_{\mathrm{r}}, \mathrm{z}, \mathrm{z}_{0}\right)=\int_{0}^{\infty} \mathrm{p}\left(\mathrm{r}, \mathrm{z}, \mathrm{z}_{0}\right) \mathrm{J}_{0}\left(\mathrm{k}_{\mathrm{r}} \mathrm{r}\right) \mathrm{r} \mathrm{dr}
\end{aligned}
$$

The transformation of the pressure field to a depth dependent Green's function is a particularly useful tool in examining the modal behavior of the waveguide. The depth dependent Green's function (which will be called the Green's function hereafter), is a function of the horizontal wavenumber, $\mathrm{k}_{\mathrm{r}}$, and the source and receiver depths, $\mathrm{z}_{0}$ and $\mathrm{z}$ respectively. It is in the examination of the horizontal wavenumber spectra that the influence of the boundaries on the modal structure is easily observed.

In chapter 2, the pressure field was also expressed as a sum of a modal portion and a continuous contribution. Current studies of the normal mode approach have shown this decomposition carries over to the Green's function in the following manner[25]:

$$
\begin{gathered}
\mathrm{p}(\mathrm{r})=\mathrm{j} \pi \sum_{\mathrm{n}=1}^{N} \mathrm{a}_{\mathrm{n}} \mathrm{k}_{\mathrm{m}} \mathrm{H}_{0}^{(1)}\left(\mathrm{k}_{\mathrm{mn}} \mathrm{r}\right)+\mathrm{p}_{\mathrm{c}}(\mathrm{r}) \\
\mathbb{g}\left(\mathrm{k}_{\mathrm{r}}\right)=\sum_{\mathrm{n}=1}^{N} \frac{2 \mathrm{a}_{\mathrm{n}} \mathrm{k}_{\mathrm{m}}}{\left(\mathrm{k}_{\mathrm{r}}^{2}-\mathrm{k}_{\mathrm{m}}^{2}\right)}+\mathrm{g}_{\mathrm{c}}\left(\mathrm{k}_{\mathrm{r}}\right)
\end{gathered}
$$

That is, the modal portion of the pressure field is directly related to the modal portion of the Green's function through a zero order Hankel transform. The coefficient, $a_{n}$, is the residue (from the Cauchy residue theorem) for a given $k_{r}$; that is, the coefficient is defined as $a_{n}=$ $\mathrm{k}_{\mathrm{r}} \rightarrow \lim _{\mathrm{m}}\left(\mathrm{k}_{\mathrm{r}}-\mathrm{k}_{\mathrm{m}}\right) \mathrm{g}\left(\mathrm{k}_{\mathrm{r}}\right)$. An alternate expression for the coefficient in terms of the vertical eigenfunctions, $u_{n}$, is[25]: 


$$
a_{n}\left(k_{r}\right)=\frac{u_{n}\left(z_{0}\right) u_{n}(z)}{\rho\left(z_{0}\right) k_{r n}}
$$

For a Pekeris waveguide, the specific coefficients may be found using:

$$
a_{n}(k r)=\frac{\sin \left(\gamma_{0} z\right) \sin \left(\gamma_{0} z_{0}\right)}{k_{r}\left(h-\frac{\sin \left(2 \gamma_{0} h\right)}{4 \gamma_{0}}+j \frac{b \sin ^{2}\left(\gamma_{0} h\right)}{2 \gamma_{1}}\right)} \mid k r=k m
$$

where

$\mathrm{b}=\frac{\gamma_{0}}{\mathrm{j} \gamma_{1} \mathrm{~h}} \quad \mid \mathrm{kr}=\mathrm{km}$ and the subscripts 0 and 1 denote the water column and half space respectively.

There are two additional items which should be noted with regard to the Green's function. The first characteristic concerns the rational form expression for the Green's function. In equation (3.1.12), the modal portion of the Green's function, $\mathrm{g}_{\mathrm{m}}$, is given as:

$$
\mathrm{g}_{\mathrm{m}}=\sum_{\mathrm{n}=1}^{\mathrm{N}} \frac{2 \mathrm{a}_{\mathrm{n}} \mathrm{k}_{\mathrm{m}}}{\left(\mathrm{k}_{\mathrm{r}}^{2}-\mathrm{k}_{\mathrm{rn}}^{2}\right)}
$$

where both the numerator and denominator of the expression have zeroes at locations other than zero and infinity. In signal processing parlance, this is a pole-zero or ARMA (autoregressive moving average) model. The zeroes of the denominator correspond to singularities and are called the poles of the system. In the above expression, the poles are expressed in terms of the horizontal wavenumber and are located at $\mathrm{k}_{\mathrm{r}}= \pm \mathrm{k}_{\mathrm{rn}}$ (as expected since the Green's function is an even function). The zeroes of the numerator are the nulls of the system and are found through the identification of the coefficients $a_{n}\left(k_{r}\right)$, which may be done analytically or numerically.

The second fact regarding the Green's function is based on the physical interpretation of equation (3.1.9). The poles of the equation are functions of the waveguide environment since the numerator is a function of $R_{b}, R_{S}, h$ and $k_{r}$ (recall the horizontal wavenumber, $k_{r}$, is related to the vertical wavenumber, $\gamma$, by $k_{\mathrm{r}}=\sqrt{\mathrm{k}^{2}-\gamma^{2}}$ ). The numerator of the Green's 
function has zeroes which are determined by the waveguide environment and the geometry of source and receiver. As the source and receiver positions are varied within a given waveguide, we expect a shift in the zero locations and a corresponding change in the null locations on a spectral plot of the Green's function. At certain source-receiver geometries, the zero will cancel a pole and the mode will not propagate. 


\subsection{Prony Energy Spectral Density}

In the last section, the modal behavior of the waveguide was available for examination through the Green's function. The Green's function is related to the pressure field through a zero order Hankel transform. Prony's method is a model parameter estimation approach rather than a spectral estimation technique. Since Prony's method estimates the system poles ( the $\mathrm{k}_{\mathrm{rn}}$ ) as one of the model variables, we wish to develop a spectral plot which incorporates this and the other parameter values.

The first step in defining a spectrum for the method is an assumption of the behavior of the data outside the processing interval. Marple presents three possible spectra which start from different interpretations of data characteristics[31]. In this study, the data is assumed to have even pressure characteristics with respect to the origin, that is, $p(r)=p(-r)$. Physically, this may be justified by considering the symmetry assumptions made in the analytical solution to the waveguide problem. In chapter 2 , a circular symmetry assumption was invoked to allow removal of $\theta$ dependency through integration. In terms of measurements, if we place our spatial array at a horizontal range $\mathrm{x}_{0}$ from the origin, we would expect the same measurements

if the array was located at $-\mathrm{x}_{0}$. Simply put, this assumption requires the effects of attenuation, spreading, etc. to be the same on either side of the origin.

This symmetry is incorporated in the spectrum by assuming a two sided function which is defined as:

$$
\begin{aligned}
y[n] & =\sum_{q=0}^{p-1} v_{q} z_{q}^{n} \quad \text { for } n \geq 0 \\
& =\sum_{q=0}^{p-1} v_{q}\left(z_{q}^{*}\right)^{-n} \quad \text { for } n<0
\end{aligned}
$$

The z-transform of this model is[51]: 


$$
\mathrm{Y}(\mathrm{z})=\sum_{\mathrm{q}=0}^{\mathrm{p}-1} \mathrm{v}_{\mathrm{q}}\left(\frac{1}{1-\mathrm{z}_{\mathrm{q}} \mathrm{z}^{-1}}-\frac{1}{1-\left(\mathrm{z}_{\mathrm{q}}^{*} \mathrm{z}\right)^{-1}}\right)
$$

Using the definitions of the roots $\mathrm{z}_{\mathrm{q}}$ and $\mathrm{z}_{\mathrm{q}}^{*}$ :

$$
\begin{aligned}
& z_{q}=\exp \left(\alpha_{q} T+j k_{q} T\right) \\
& \left(z_{q}^{*}\right)^{-1}=\exp \left(-\alpha_{q} T+j k_{q} T\right)
\end{aligned}
$$

and assuming the spectral radius of the poles is less than one, a discrete time Fourier transform (DTFT) may be found. The DTFT is:

$$
\begin{aligned}
& S_{1}\left(k_{r}\right)=\left.T Y(z)\right|_{z=\exp (j k r T)} \\
& =\sum_{\mathrm{q}=0}^{\mathrm{p}-1} \mathrm{v}_{\mathrm{q}}\left[\frac{\mathrm{T}\left[\exp \left(\alpha_{\mathrm{q}} \mathrm{T}\right)-\exp \left(-\alpha_{\mathrm{q}} \mathrm{T}\right)\right] \exp \left(\mathrm{j}\left[\mathrm{k}_{\mathrm{rq}}-\mathrm{k}_{\mathrm{r}}\right] \mathrm{T}\right)}{1-\left[\exp \left(\alpha_{\mathrm{q}} \mathrm{T}\right)-\exp \left(-\alpha_{\mathrm{q}} \mathrm{T}\right)\right] \exp \left(\mathrm{j}\left[\mathrm{k}_{\mathrm{rq}}-\mathrm{k}_{\mathrm{r}}\right] \mathrm{T}\right)+\exp \left(j 2\left[\mathrm{k}_{\mathrm{rq}}-\mathrm{k}_{\mathrm{r}}\right] \mathrm{T}\right)}\right]
\end{aligned}
$$

The energy spectral density (ESD) is found by the magnitude squared of the DTFT, ie $S\left(k_{\mathrm{r}}\right)=$ $\left|\mathrm{S}_{1}\left(\mathrm{k}_{\mathrm{r}}\right)\right|^{2}$. We will refer to this function as the ESD in the rest of the thesis.

The $\mathrm{z}$ transform of the two sided function demonstrates a characteristic of the Prony method; the model is an all pole system. Rewriting the $\mathrm{z}$ transform of equation (3.2.1) in terms of a common denominator:

$$
\begin{aligned}
Y(z) & =\sum_{\mathrm{q}=0}^{\mathrm{p}-1} \mathrm{v}_{\mathrm{q}}\left(\frac{1-\left(\mathrm{z}_{\mathrm{q}}^{*} \mathrm{z}^{-1}-\left(1-\mathrm{z}_{\mathrm{q}} \mathrm{z}^{-1}\right)\right.}{\left(1-\mathrm{z}_{\mathrm{q}} \mathrm{z}^{-1}\right)\left(1-\left(\mathrm{z}_{\mathrm{q}}^{*} \mathrm{z}^{-1}\right)\right.}\right) \\
& =\sum_{\mathrm{q}=0}^{\mathrm{p}-1} \mathrm{v}_{\mathrm{q}}\left[\frac{\left[\mathrm{z}_{\mathrm{q}}-\left(\mathrm{z}_{\mathrm{q}}^{*}\right)^{-1}\right] \mathrm{z}^{-1}}{\left(1-\mathrm{z}_{\mathrm{q}} \mathrm{z}^{-1}\right)\left(1-\left(\mathrm{z}_{\mathrm{q}}^{*} \mathrm{z}^{-1}\right)\right.}\right]
\end{aligned}
$$

In the above representation, it is readily apparent the numerator of the $\mathrm{z}$ transform has no zeroes except at zero and infinity for all $\mathrm{q}$. 
The all pole nature of the Prony ESD dictates the appearance of the spectral plot. The singularities of the system, the poles, yield a typical spectrum which has sharp peaks. In contrast, an all zero (MA or FIR) filter has sharp nulls. The pole-zero model (ARMA) has both sharp nulls and peaks in the spectral plot[51].
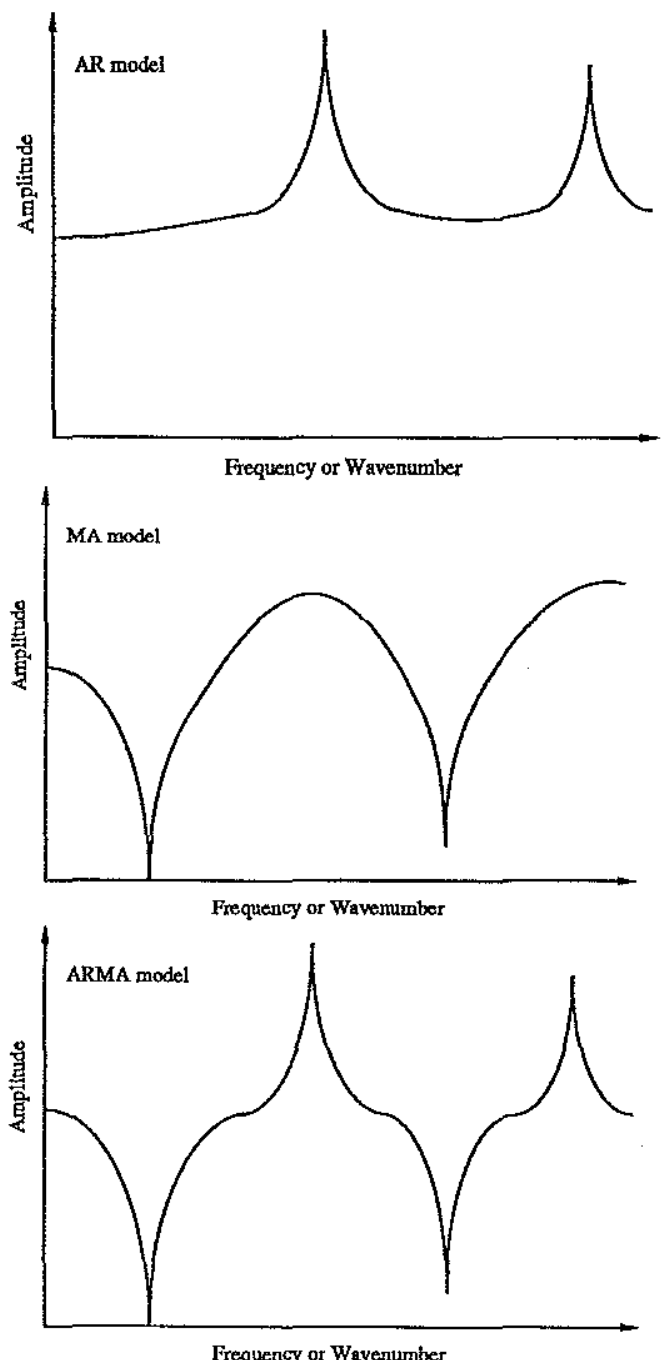

Fig 3.2.1 Comparison of typical AR, MA and ARMA spectra 
In the last section, the pole-zero nature of the Green's function was evident. Indeed, a plot of the Green's function for synthetically generated data confirms this characteristic (see, for example, fig 3.3.3). Instead of taking a Hankel transform of the pressure field, the Prony ESD is achieved by a DTFT of the square root of the range times the modal portion of the discrete pressure field. While the ESD of the resulting all pole model not the Green's function, it is related to it. The Wold decomposition theory states that we may model an ARMA or MA filter with an infinite order AR filter[32]. Given the constraint of a finite filter, the relationship between the AR filter coefficients and the ARMA coefficients and the AR filter length should be explored. Consider the Green's function as an ARMA model of $\mathrm{s}$ poles and $\mathrm{r}$ zeroes. Then, using the analogy of a filter driven by a unit impulse:

$$
H(z)=\frac{B(z)}{D(z)}=\frac{\sum_{m=0}^{r} b[m] z^{-m}}{\sum_{q=0}^{s} a[q] z^{-q}}
$$

with $b[0]=a[0]=1$. To match this with an all pole filter specified by:

$$
\mathrm{V}(\mathrm{z})=\frac{1}{\mathrm{G}(\mathrm{z})}=\frac{1}{\sum_{\mathrm{w}=0}^{\infty} \mathrm{g}[\mathrm{w}] \mathrm{z}^{-\mathrm{w}}}
$$

requires $\mathrm{H}(\mathrm{z})=\mathrm{V}(\mathrm{z})$. Substituting the above expressions:

$$
\frac{\mathrm{B}(\mathrm{z})}{\mathrm{D}(\mathrm{z})}=\frac{1}{\mathrm{G}(\mathrm{z})} \Rightarrow \mathrm{B}(\mathrm{z}) \mathrm{G}(\mathrm{z})=\mathrm{D}(\mathrm{z})
$$

The multiplication in the $\mathrm{z}$ domain is equivalent to convolution in the time domain, so:

$$
\mathrm{b}[\mathrm{n}] * \mathrm{~g}[\mathrm{n}]=\mathrm{d}[\mathrm{n}] \Rightarrow \sum_{\mathrm{k}=-\infty}^{\infty}(\mathrm{b}[\mathrm{k}] \mathrm{g}[\mathrm{n}-\mathrm{k}])=\mathrm{d}[\mathrm{n}]
$$

Since there are $s$ poles in the ARMA model, $d[n]=0$ for $n>s$ and

$$
\begin{aligned}
\mathrm{g}[\mathrm{n}] & =-\sum_{\mathrm{k}=1}^{\mathrm{r}}(\mathrm{b}[\mathrm{k}] \mathrm{g}[\mathrm{n}-\mathrm{k}])+\mathrm{d}[\mathrm{n}] & & 0<\mathrm{n} \leq \mathrm{s} \\
& =-\sum_{\mathrm{k}=1}^{\mathrm{r}}(\mathrm{b}[\mathrm{k}] \mathrm{g}[\mathrm{n}-\mathrm{k}]) & & \mathrm{n}>\mathrm{s}
\end{aligned}
$$


This expression assumes $\mathrm{g}[\mathrm{n}]$ is causal and $\mathrm{g}[0]=1$. The validity limits for the expressions are due to the number of ARMA poles while the number of ARMA zeroes defines the convolution length. In the AR approximation, these $\mathrm{g}[\mathrm{n}]$, the all pole filter coefficients, are set equal to zero for $\mathrm{n}>\mathrm{r}+\mathrm{s}$. As shown above, the poles and zeroes of the ARMA filter influence the output's first s points. After that, only the poles influence the system output. To determine the ARMA parameters given an AR approximation, the above equations may be used to find the numerator and denominator terms using $r+s+1$ output points:

$$
\left(\begin{array}{cccc}
c[s] & c[s-1] & \ldots & c[s-r+1] \\
c[s+1] & c[s] & \ldots & c[s-r+2] \\
\vdots & : & : & : \\
c[s+r-1] & c[p+q-2] & \ldots & c[p]
\end{array}\right)\left(\begin{array}{c}
b[1] \\
b[2] \\
\vdots \\
b[r]
\end{array}\right)=-\left(\begin{array}{c}
c[p+1] \\
c[p+2] \\
\vdots \\
c[p+q]
\end{array}\right)
$$

and

$$
a[n]=c[n]+\sum_{k=1}^{r} b[k] c[n-k]
$$

In other words, the expressions for $\mathrm{g}[\mathrm{n}]$ are forcing these coefficients to match the first $\mathrm{r}+\mathrm{s}+1$ coefficients of the infinite length inverse ARMA polynomial $\mathrm{X}(\mathrm{z})$ where

$$
X(z)=\frac{1}{H(z)}=\frac{D(z)}{B(z)}=\sum_{k=0}^{\infty} x[k] z^{-k} \text {. }
$$

The last section contained a description of the Green's function as a superposition of a modal and continuous portion. The modal sum in the Green's function may be expressed by[25]:

$$
g_{m}\left(k_{r}\right)=\sum_{n=1}^{N} \frac{2 a_{n} k_{m}}{\left(k_{r}^{2}-k_{r n}^{2}\right)}
$$

where $\quad a_{n}=\frac{u_{n}\left(z_{0}\right) u_{n}(z)}{\rho\left(z_{0}\right) k_{r n}}$

This may be cast in the form of an ARMA model with $k_{r}$ replaced by $z$. However, the expression for the coefficients $\mathrm{a}_{\mathrm{n}}$ is, in general, not trivial and requires substitutions and/or 
series expansions to yield a polynomial form in the numerator. A direct comparision of the Green's function and Prony ESD is not useful; the quantities are not the same since the Green's function is an ARMA process and the Prony ESD is an AR process. The poles of the Green's function will, however mathc those of the Prony ESD; the ESD will also yield relative energy levels of the system modes. The Green's function is related to the pressure field by a zero order Hankel transform while the Prony ESD is related to the square root of the range times the pressure field by a discrete time Fourier transform.

The ESD uses all of the information generated by the Prony estimation algorithm. This presentation has two distinct advantages. First, the tabular form is transformed to a graphical representation. The total effect of all model parameters is summarized in a concise output which allows easy assimilation of the algorithm output. Second, the ESD is a tool which allows comparision of range blocks. The present algorithm has no constraints on continuity of parameter values between subsequent range blocks; each block is evaluated as a "stand alone" entity. Changes in bathymetry and waveguide boundaries may be observed in shifts in the ESD peaks and levels between blocks of interest. This range dependent performance is further explored in the next chapter. 


\subsection{Algorithm Performance in Range Independent Waveguides}

In this section, we will explore the application of the Prony algorithm to a synthetically generated pressure field. The algorithm explored was outlined in chapter two; in brief, it is a three step process. The first step consists of obtaining a least squares (LS) fit of the polynomial coefficients for a specified model order. After using a $Q R$ decomposition to obtain these coefficients, a numerical rooting program finds the roots of the polynomial. The last step is the LS fit of the data to the remaining model parameters. The parameters specified by the users include the starting and stopping range, the model order, the processing block size, processing block overlap and averaging block size. The relationship of the last three variables is outlined below and in figure 3.3.1.

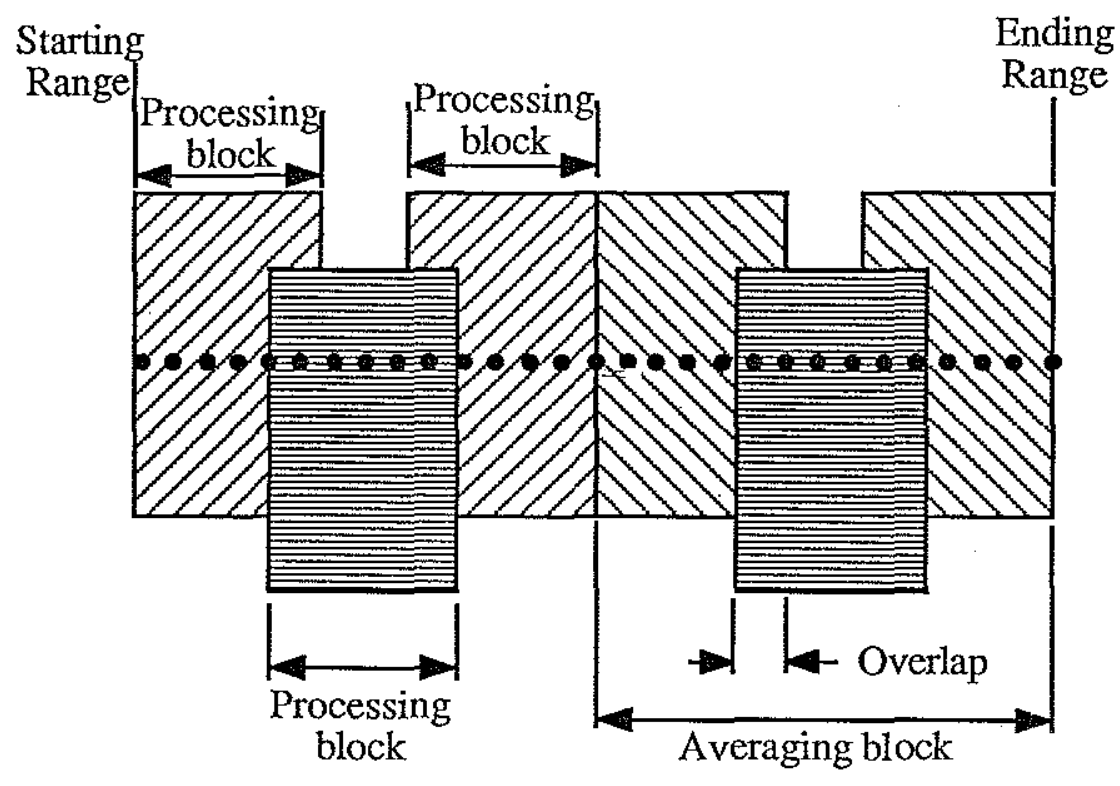

Fig 3.3.1 Range aperture block constituents

The processing block is the number of points used for each three step iteration of the method.

The processing block overlap allows the user to set the starting and stopping ranges of each block so that adjacent blocks may overlap one another. The averaging block size permits the 
parameter values within a given range interval to be averaged together. In figure 3.3.1, an averaging block is shown with three processing blocks overlapping by one third. It should be emphasized that this is not an averaging of spectra as is usually encountered in the signal processing literature. Rather, the averaging is among the parameter values; it takes place before the ESD is calculated.

Before exploring the utility of the ESD in evaluating the Prony algorithm performance, we will describe another analysis tool which may be used to obtain a quantitative assessment of the modelling process. As shown in figure 3.3.2 below, the observed data (the square root of the range times the sampled pressure field) is assumed to be the output of an ARMA filter. The all pole filter of Prony's method uses the observed data, y[n], to estimate the Prony filter coefficients, $\mathrm{V}(\mathrm{z})$. Given the all pole filter, $\mathrm{V}(\mathrm{z})$, we want to develop a method of assessing how well the actual data fits the all pole model. If s[n], the output of the Prony filter, was used as input of the inverse filter, $G(z)=\frac{1}{V(z)}$, the output, $r[n]$, would be a unit impulse[51]. Instead of using $s[n]$ as input to $\mathrm{G}(\mathrm{z})$, the observed data is used. If $\mathrm{H}(\mathrm{z})$ was, in fact, all pole, and matched by $\mathrm{V}(\mathrm{z})$, then the output of $\mathrm{G}(\mathrm{z})$ would be an impulse of height equal to the gain term of $\mathrm{H}(\mathrm{z})$ (because $\overline{\overline{\mathrm{V}}}(\mathrm{z})$ assumes a gain of 1 ). If $\mathrm{H}(\mathrm{z})$ is an ARMA process and/or there is noise in the system, then using $y[n]$ as input to the inverse filter will yield a data sequence which has nonzero terms at other than the origin. The residue is the normalized total energy of this sequence: $\mathrm{R}=\frac{1}{\mathrm{~N}} \sum_{\mathrm{n}=1}^{\mathrm{N}} \mathrm{r}[\mathrm{n}] \mathrm{r}^{*}[\mathrm{n}]$. The smaller the residue, the better the actual data fits an all pole model since there is less energy in the residual. The last statement requires a qualification for completeness. The residue information yields a quantitative measurement of observed data fit to an all pole model; it makes no statement regarding the accuracy of the parameter values to the actual waveguide values. 


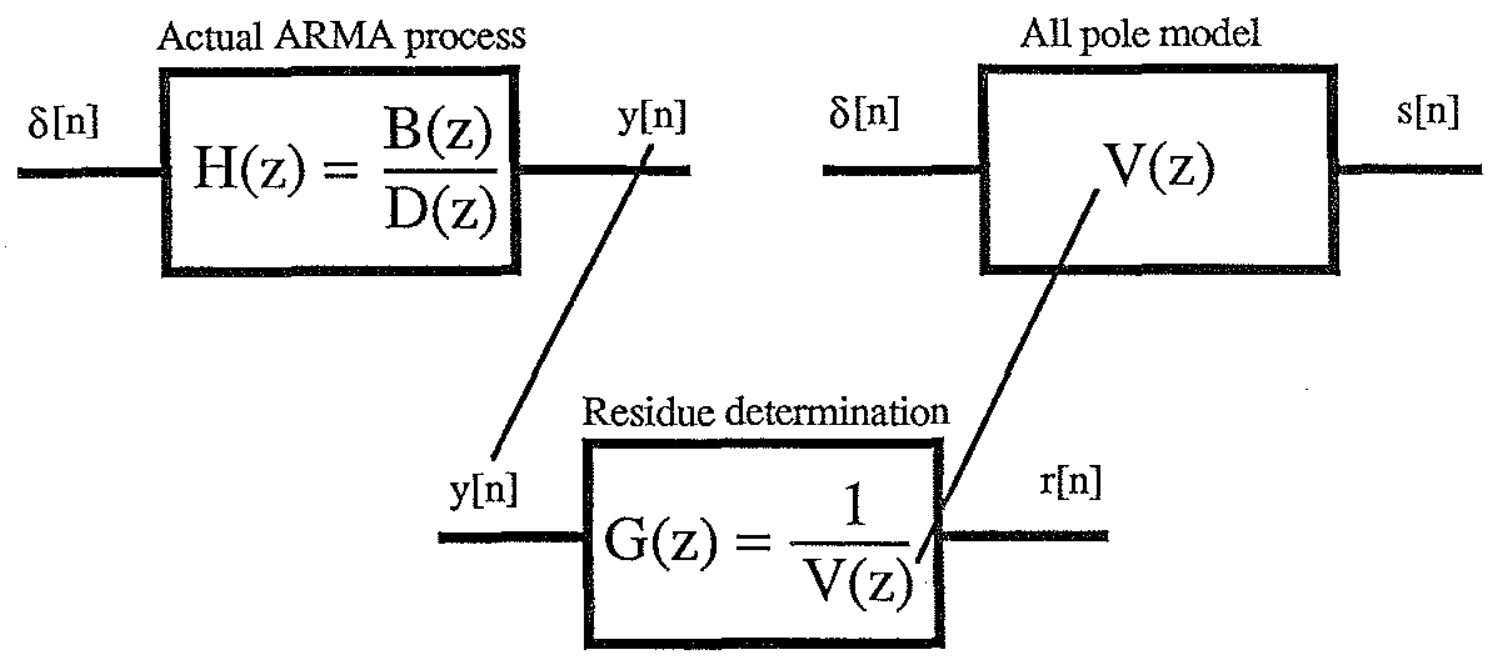

Fig 3.3.2 Residue determination

With these caveats, it is acceptable to add the residue to the performance evaluation tools. The four tools which will be used are the ESD, residue, wavenumber vs. range and pole positions in the complex $\mathrm{z}$ plane. Each of these serves to highlight specific characteristics of the waveguide performance. The ESD is best used to summarize the modal behavior of the system including range dependent features (which will be covered in chapter 4). The residue and wavenumber plots are useful for determining when a "good" system order is reached and the pole plots assist in identifying actual modes from the arbitrary system poles.

One of the classical difficulties facing the researcher implementing Prony's method is the specification of a model order. This parameter is distinct from the system order. The system order (the number of modes actually propagating) may be obtained by inspection or numerically. The Green's function (which is generated by numerically Hankel transforming the pressure field) may be used to estimate the number of system modes. However, large apertures are necessary for accurate discrete Hankel transform results. To numerically extract the number of system modes, an SVD of the signal matrix is the recommended procedure[36,37,43-44]. Ordering of the singular values by magnitude will illustrate the drop in singular value magnitude for singular values greater than the actual system order (ie, if the 
model order is ten and the system order is six, the first six largest singular values will be significantly higher than the remaining singular values). The addition of noise to the system complicates both of these procedures.

The specification of a "good" model order is more of an art. If the order specified is too low, the resulting parameter estimation will be poor as the system energy is constrained to be distributed among the specified order. Empirically, this situation results in the identification of some of the actual modes, generally the strongest (which are determined by source-receiver geometry). The sytem description is, nonetheless, incomplete. If, on the other hand, the model order is too high, the output may contain spurious peaks and/or the signal matrix may be singular[32]. While the noise and non modal components of the energy field (the continuum) may prevent the matrix from actually becoming singular, the matrix becomes more ill conditioned as order increases. This may make numerical determination of the actual system order easier since the larger singular values get larger and the smaller singular values get smaller near singularity[40,41]. Since we do not specifically use the actual system order, this small positive feature of largely overspecified models is negated by the problems associated with decomposition of the ill conditioned matrix. The spurious peaks are usually of low energy and have little effect. In some trials, the small but finite energy in these poles robbed the valid modes of energy resulting in a poorer fit of data to an all pole model. The development of an analytical method to determine an appropriate model order is a current research effort. In a recent paper, Braun and Ram describe an SVD approach which yields effective results especially in noisy situations[6].

The approach used in this study to examine the effect of model order on parameter estimation was iterative in nature. Two different schemes were used. In the first, the model order was increased and the total residue for the range interval was obtained. The residue dropped to a plateau after a certain amount of overdetermination. The second scheme examined parameter "wander" with respect to increases in model order. Both pole plots and wavenumber 
vs. range plots were examined with increases in model order. Initially, if the model was underspecified in order, the wavenumbers and other parameters were found to change slightly as the order was increased. At a certain point in overspecification, the wavenumber change was negligible for increase in model order. The pole plot proved to be more effective in determining actual modes rather than in assisting in parameter wander identification.

In exercising the algorithm, two bottom models were chosen. The first was a Nantucket type bottom with source frequencies of 140 and $220 \mathrm{~Hz}$. The second bottom profile was similar to the type of bottom found off the coast of Corpus Christi with source frequencies of 50 and $140 \mathrm{~Hz}$. The bottom models were chosen because they approximated the experimental environments. The sound fields were generated using the SAFARI code which utilizes a propagator matrix approach[55,56]. Unlike SNAP, continuum contributions are addressed by SAFARI. Because of the continuum contribution, we expect the all pole model not to fit the pressure field as well as a SNAP generated data set. The continuum effects are near field; at long ranges, their effect should be quite small.

In the Nantucket model, the bottom profile of figure 2.3.1 was used with an attenuation of $0.07 \mathrm{~dB} / \lambda$. The three propagating modes are well defined and widely spaced; this bottom is not particularly sensitive to model parameter variations in model order, processing block, averaging or overlap. Nonetheless, the bottom model serves to illustrate some basic performance aspects of the algorithm in a "realistic" (but still noisefree) environment. The ESD provides a useful tool for the overall modal behaivor of the waveguide. In this respect, the ESD is comparable to the Green's function (figure 3.3.3) since it indicates energy in the various modes. In figure 3.3.4, a small aperture model is used to estimate the parameters for the ESD. The total number of points needed for each range block is 15 points at 3.2 meter spacing. 


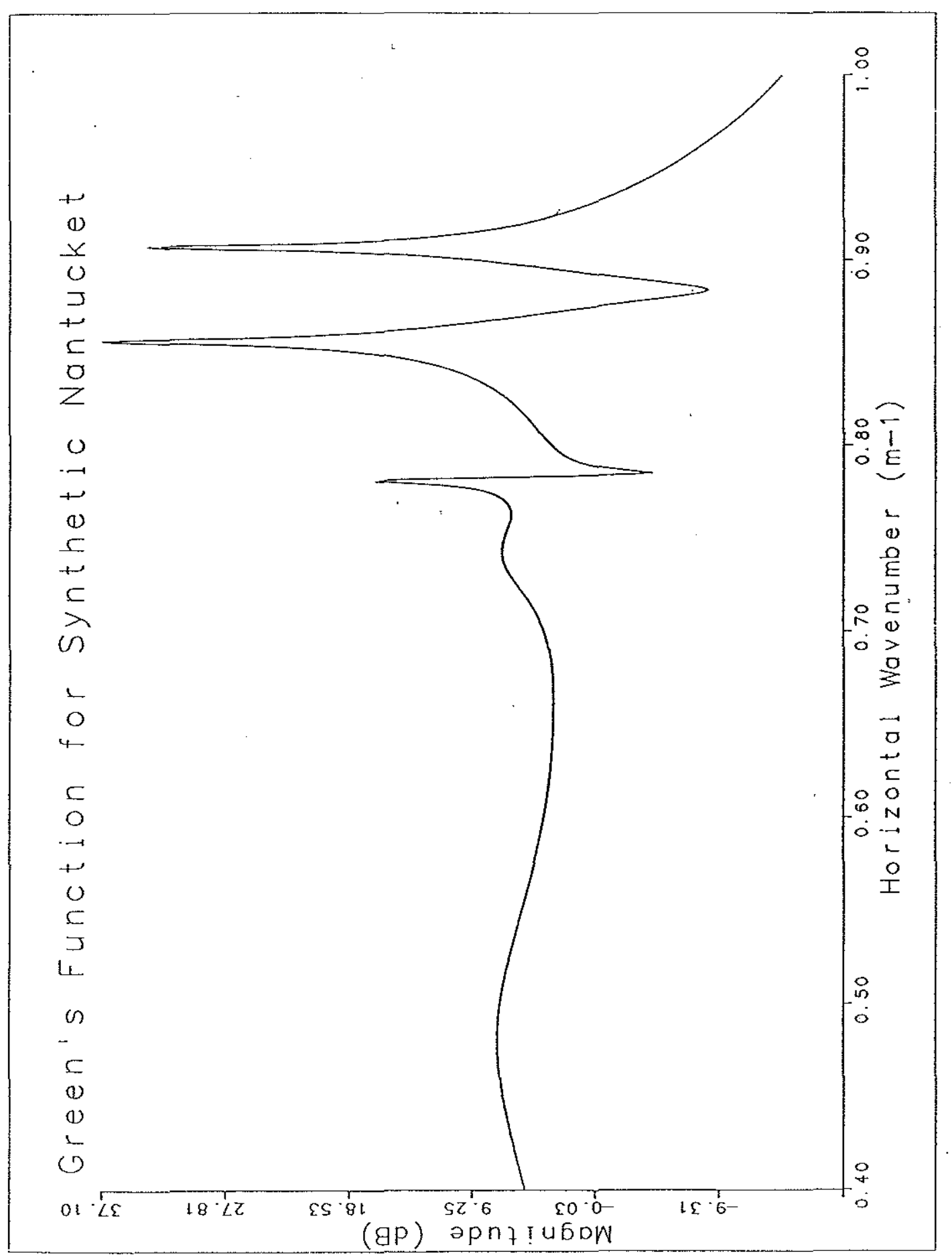

Fig 3.3.3 Green's function of Nantucket profile 


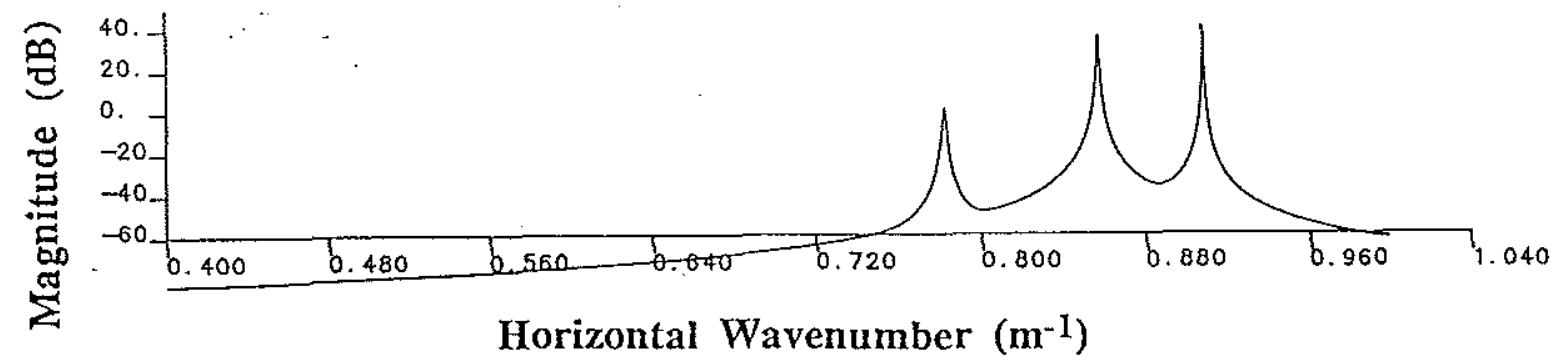

Fig 3.3.4 ESD of PRAWNS output on Nantucket profile at $4000 \mathrm{~m}$ (15 points, 0 avg, $3.2 \mathrm{~m}$ spacing, model order 7)

The accuracy of the PRAWNS algorithm output vs the Green's function pole locations is best seen in table 3.3.1. The Green's function pole locations were found through use of a peak finding routine. The PRAWNS wavenumbers are the tabular values of the model specified in figure 3.3.4 at 4000 meters. The range of 4000 meters refers to the first processing block which contains that user specified range; the 4000 meter point may occur anywhere within this processing block. This manner of specifying a range will be the convention in the rest of this thesis.

Table 3.3.1 Comparision of PRAWNS and Green's function pole locations (Nantucket profile)

\begin{tabular}{|c|c|c|}
\hline Mode & Horizontal W: & avenumber $\left(\mathrm{m}^{-1}\right)$ \\
\hline & PRAWNS & Green's Function \\
\hline 1 & 0.9077464 & 0.907701 \\
\hline 2 & 0.8569943 & 0.856988 \\
\hline 3 & 0.7822649 & 0.782204 \\
\hline
\end{tabular}

The Nantucket profile provides the opportunity to demonstrate the utility of the pole plots. This is a $\mathrm{Z}$ plane representation of the wavenumber and damping parameters using the information generated as follows: 
Pole magnitude $=\sqrt{Z_{\text {real }}{ }^{2}+Z_{\text {imag }}{ }^{2}}$

Pole angle $=\tan ^{-1}\left(\frac{Z_{\text {imag }}}{Z_{\text {real }}}\right)$

where

$$
\begin{aligned}
& \mathrm{Z}_{\text {real }}=\mathrm{e}^{\text {damping } * \mathrm{~T}} \cos (\text { wavenumber } * \mathrm{~T}) \\
& \mathrm{Z}_{\text {imag }}=\mathrm{e}^{\text {damping }} * \mathrm{~T} \sin (\text { wavenumber } * \mathrm{~T})
\end{aligned}
$$

for each damping and wavenumber term. Identifying valid modes among the arbitrary poles estimated by the overspecified system is performed by recognizing that the valid modes won't change with variations in order while the arbitrary modes will. Figure 3.3.5 illustrates the model poles for various orders and the overlap situation which allows pole identification.

The use of residues as a method of determining a "good" model order was not particularly successful in this example. A plot of the total residue versus model order is given in figure 3.3.6. Note the drop in residue level after model order 15. This would indicate that the parameter estimation is closest to an all pole model after this order. However, it says nothing regarding the accuracy of the parameters. 
7 mode pole plot

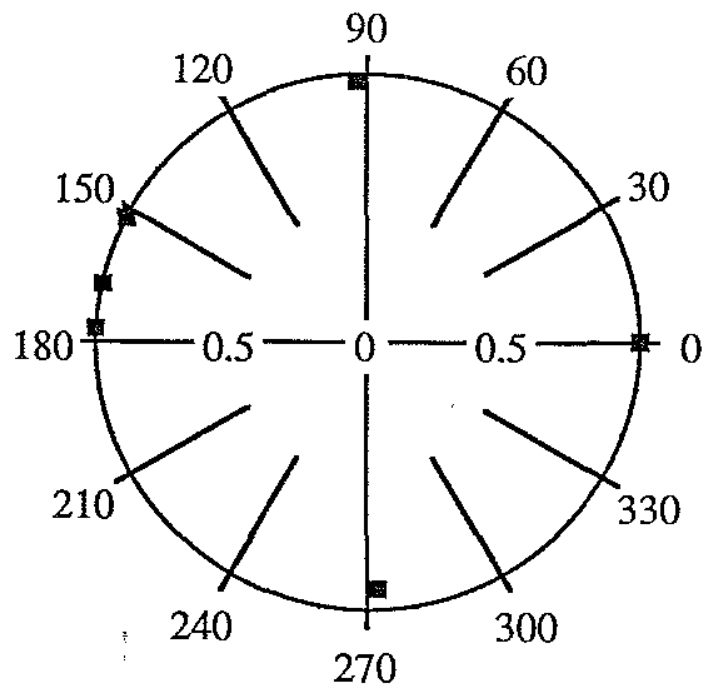

20 mode pole plot

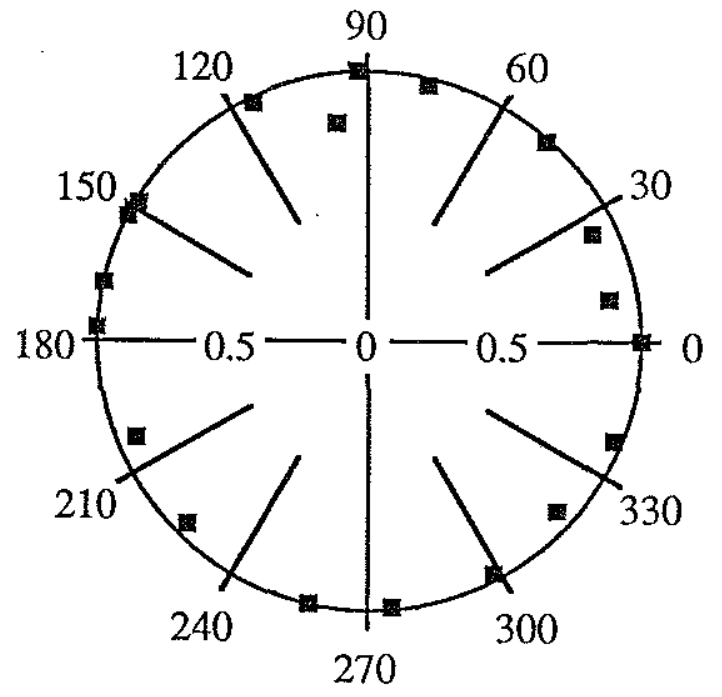

Fig 3.3.5 Comparision of pole plots for model orders 7 and 20 (Nantucket profile,50 pt, $3.2 \mathrm{~m}$ spacing, 0 overlap) 


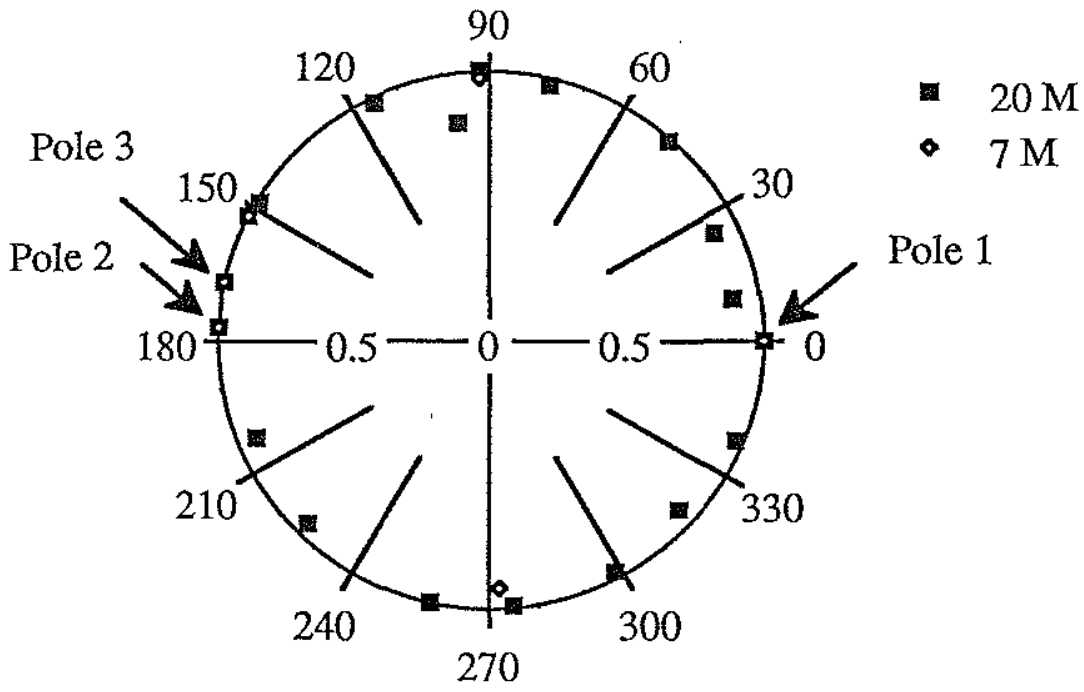

Fig 3.3.5 (continued) Comparision of pole plots for model orders 7 and 20 (Nantucket profile,50 pt, $3.2 \mathrm{~m}$ spacing, 0 overlap)

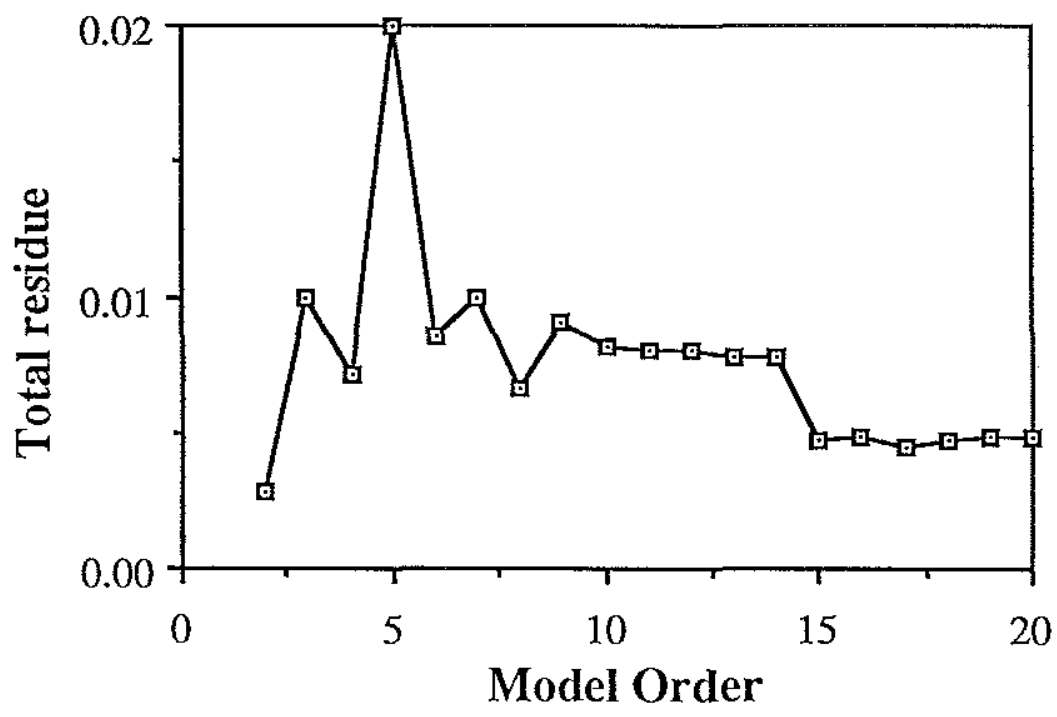

Fig 3.3.6 Total residue vs. model order for Nantucket profile (50 pt, 3.2 m spacing, 0 overlap)

An examination of the wavenumber for a particular mode as model order varies demonstrates the conservative nature of using the breakpoint to determine overspecification. Figure 3.3.7 demonstrates the need for overspecifying model order. The wavenumber of the third mode for 
a fourth order model is erratic and does not agree with the wavenumbers of the higher order model.

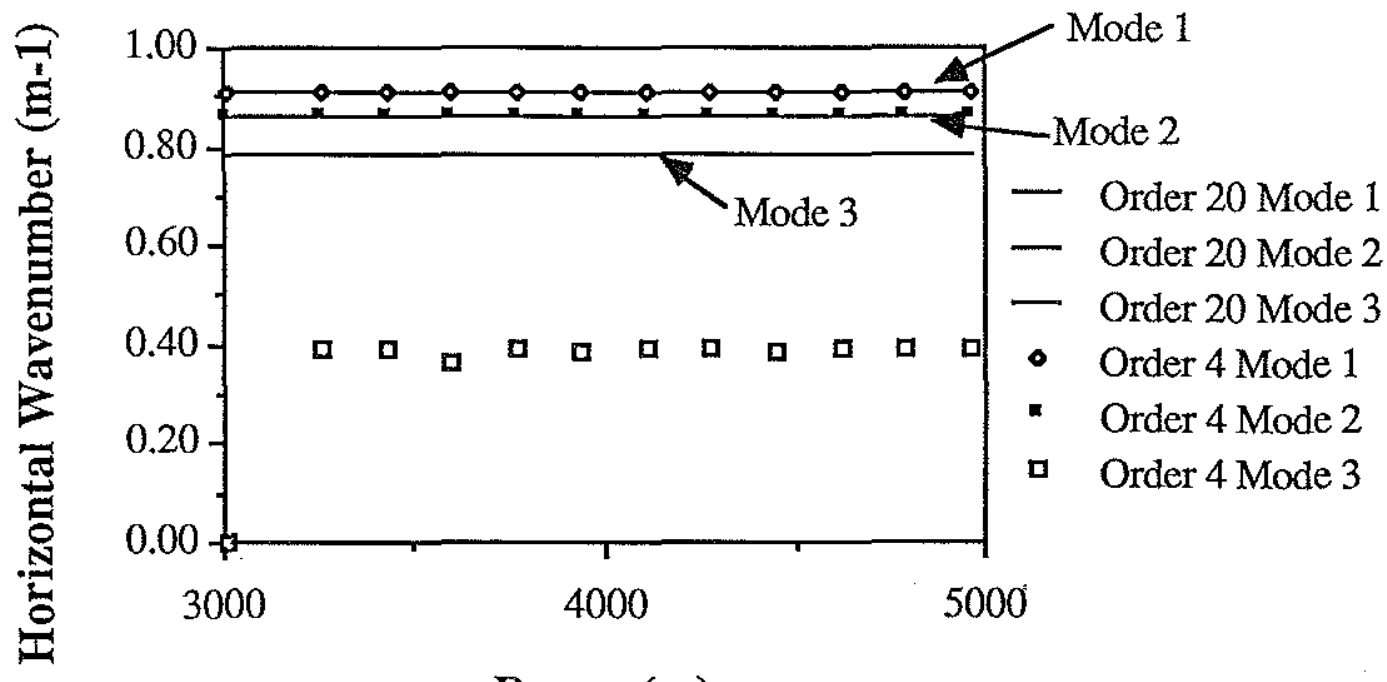

\section{Range (m)}

Fig 3.3.7 Comparision of wavenumbers for model orders 4 and 20 (Nantucket profile,50 pt, $3.2 \mathrm{~m}$ spacing, 0 overlap)

While figure 3.3.7 indicates the need to overspecify, we can see in figure 3.3.8 that the model order need not be greater than 15 to obtain excellent wavenumber estimation.of using the residue to mark the breakpoint for specifying the amount of overdetermination of model order.

The Nantucket pressure field was found to be insensitive to the amount of overlap, averaging and aperture. The use of overlap and averaging is an aid in parameter estimation in noise; since the system was not corrupted by noise, the averaging scheme had no effects on the output. The absence of noise is responsible for the wide tolerance in aperture size. The effects of the continuum are quite small at the range of interest $(3000 \mathrm{~m})$; the system was essentially a pure normal mode system. 


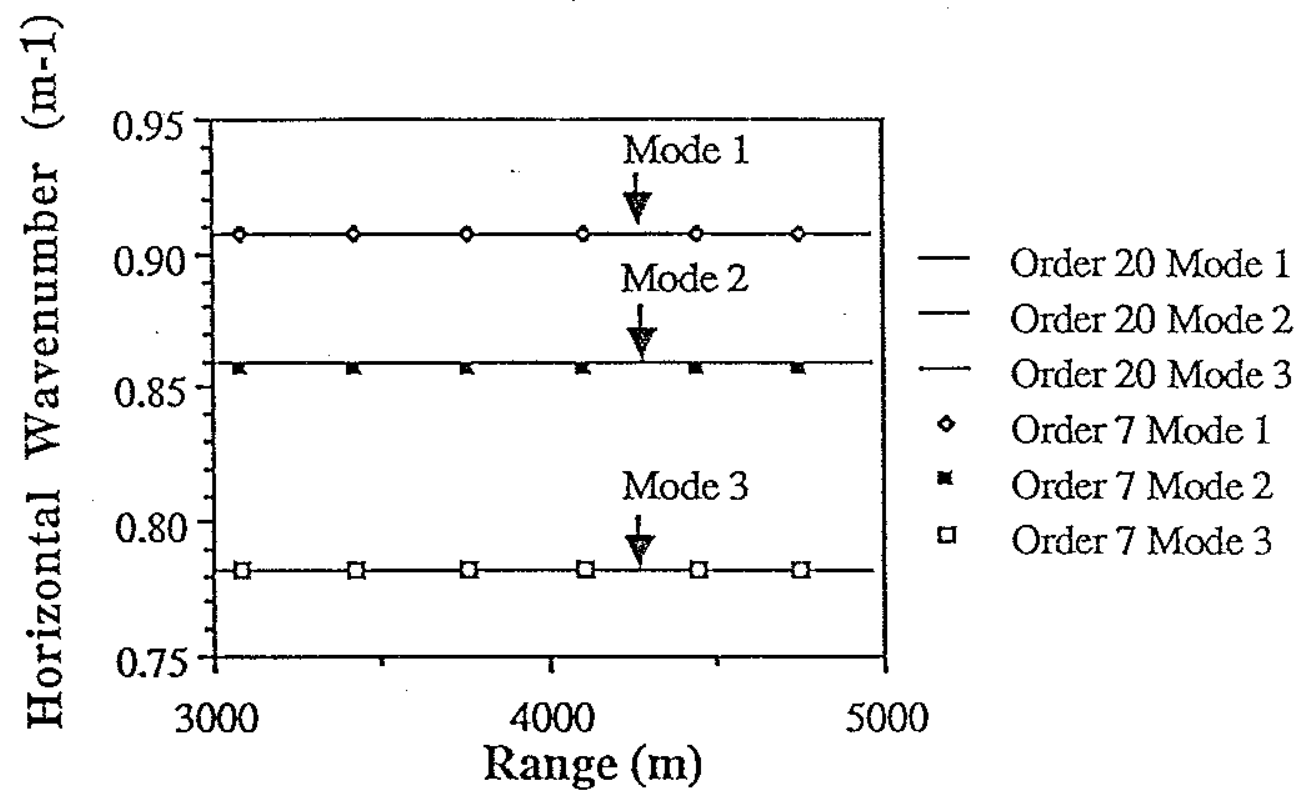

Fig 3.3.8 Comparision of 7 and 20 order model wavenumbers (Nantucket profile, $50 \mathrm{pt}, 3.2 \mathrm{~m}$ spacing, 0 overlap)

In the Corpus Christi model (fig 3.3.9), a deeper water depth was used (30 m) with a bottom density of $1.56 \mathrm{~g} / \mathrm{cm}^{3}$ and bottom attenuation of $0.07 \mathrm{db} / \lambda$. The resulting modes are closely spaced and more numerous. The selection of parameters was more critical in this situation than for the Nantucket case. Using an aperture of $340 \mathrm{~m}$ (100 pts at $3.4 \mathrm{~m}$ spacing) and model order 15, the ESD of figure 3.3.11 is compared to the Green's function of the pressure data in figure 3.3.10. 


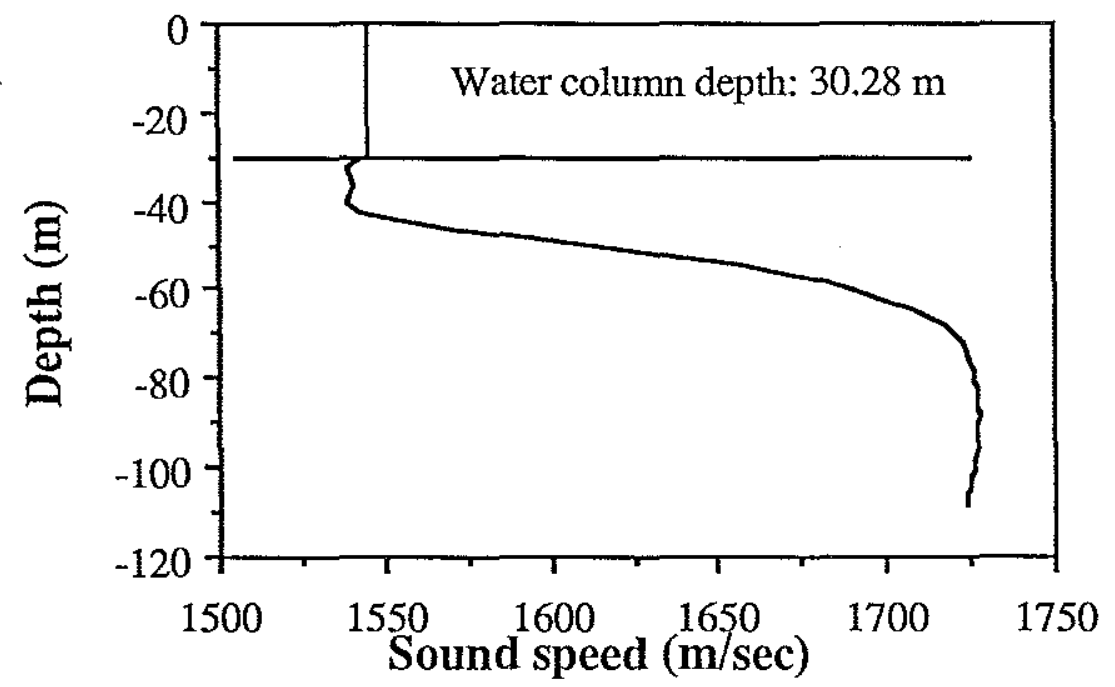

Fig 3.3.9 Corpus Christi SVP

The ESD provides a quick method of examining the overall effects of a parameter change. In figure 3.3.12, the aperture is reduced to 50 points with all other parameters constant. The six modes of figure 3.3.11 have been reduced to three. In some cases, the number of points may be increased by performing a spline fit with a finer grid. 


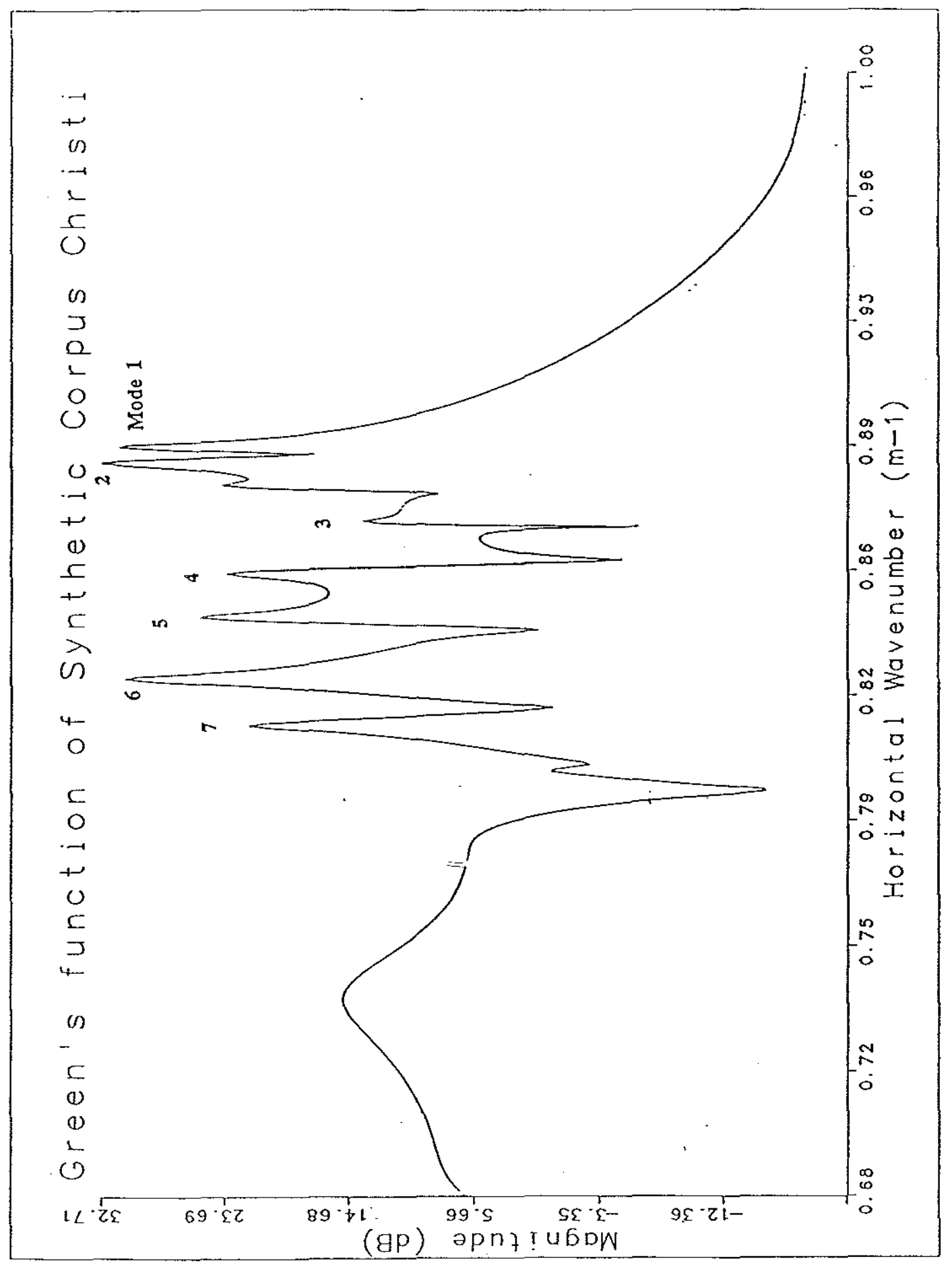

Fig 3.3.10 Green's function for Corpus Christi profile 


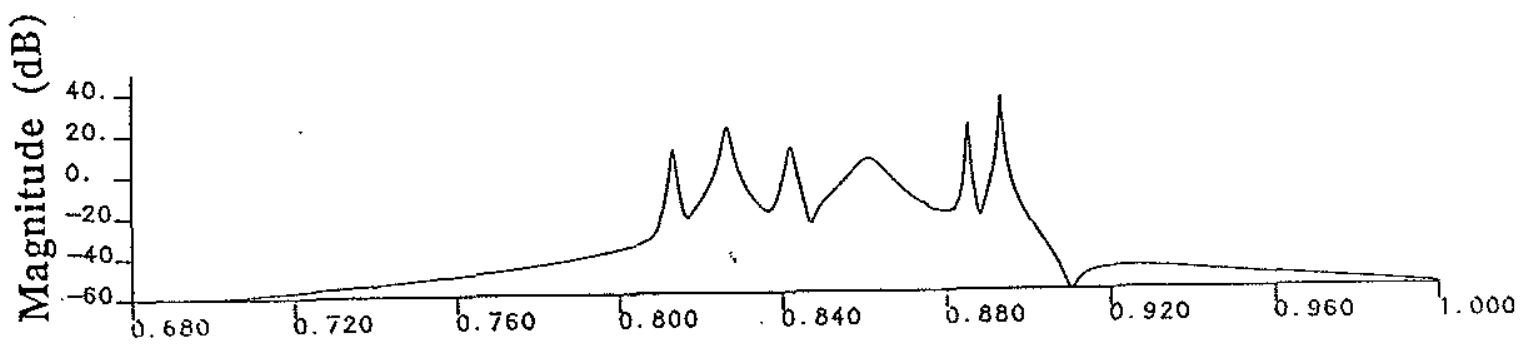

Horizontal Wavenumber $\left(\mathrm{m}^{-1}\right)$

Fig 3.3.11 ESD of 100 point sample at $4000 \mathrm{~m}$ for Corpus Christi profile

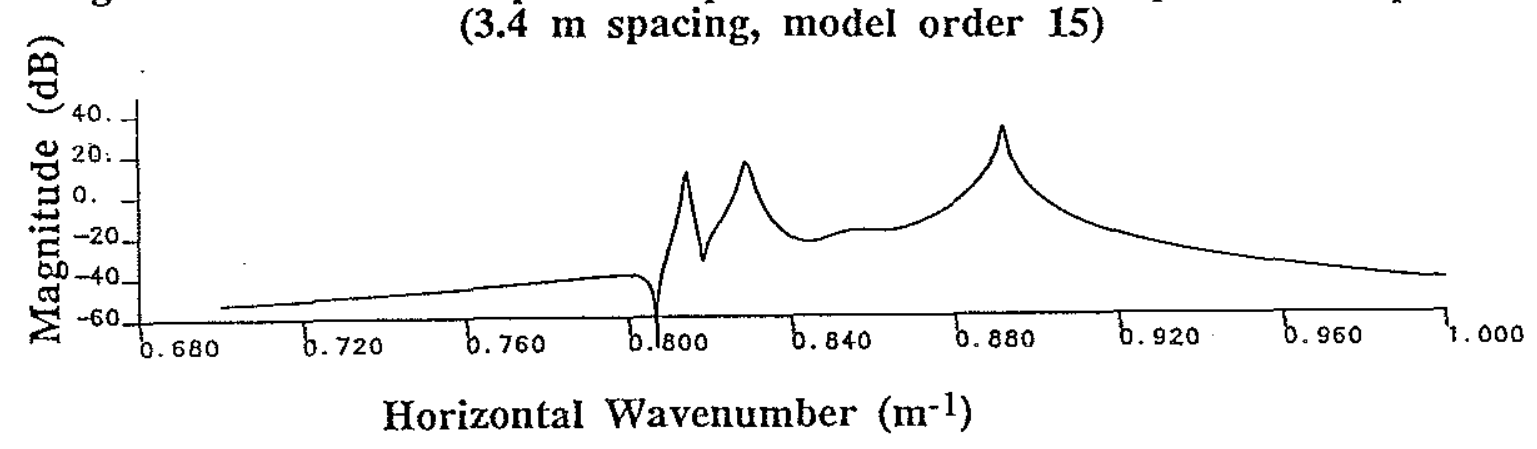

Fig 3.3.12 ESD of 50 point sample at $4000 \mathrm{~m}$ for Corpus Christi profile (3.4 m spacing, model order 15 )

Table 3.3.2 summarizes the accuracy of the PRAWNS wavenumber estimates compared to the Green's function. As in the Nantucket case, the Green function peaks were found through a peak searching routine. The PRAWNS wavenumbers are taken at $4000 \mathrm{~m}$ of a model order 24,100 point trial. 
Table 3.3.2 Comparision of PRAWNS and Green's function pole locations (Corpus Christi profile)

\begin{tabular}{ccc} 
Mode & \multicolumn{2}{c}{ Horizontal Wavenumber $\left(\mathrm{m}^{-1}\right)$} \\
& PRAWNS & Green's Function \\
1 & 0.8932641 & 0.892875 \\
2 & 0.8857910 & 0.886250 \\
3 & 0.8708455 & 0.872031 \\
4 & 0.8505248 & 0.869968 \\
5 & 0.8283763 & 0.828010 \\
6 & 0.8137148 & 0.813453 \\
7 & 0.8098074 & 0.803125
\end{tabular}

In the beginning of the section, mention was made of the iterative methods used to estimate model order. The Nantucket profile was not particularly sensitive to model order spêcification. The Corpus Christi profile[8] provides an opportunity to explore the agreement between the two methods. The first approach in model order determination involved measuring the total residue for a range interval as the model order is increased. The residue is expected to drop to a lower level after a specific model order. This order represents the breakpoint; any model order higher than this should yield good results. As we have pointed out previously, a good overall fit to an all pole model does not necessarily imply accurate parameter estimations but the method empirically does yield valid results as far as model order selection is concerned. Figure 3.3.13 illustrates the application of this technique on an $340 \mathrm{~m}$ aperture (100 points at $3.4 \mathrm{~m}$ spacing) for an arbitrary range interval. 


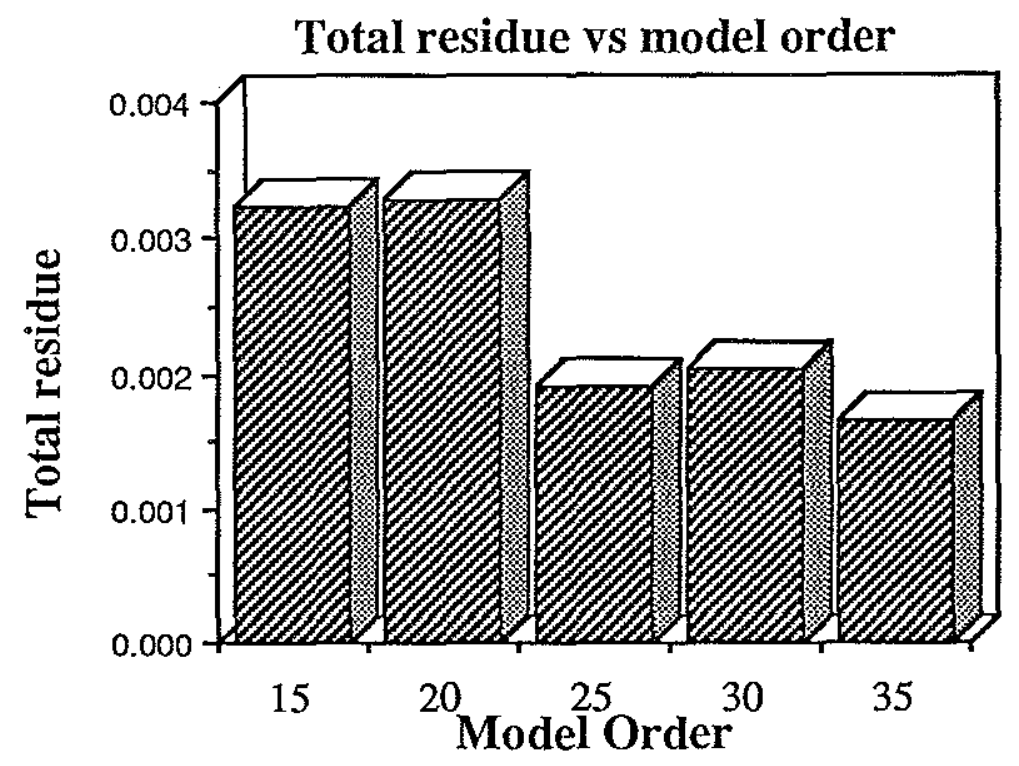

\section{Fig 3.3.13 Total residue vs model order for Corpus Christi profile (100 pt, $3.4 \mathrm{~m}$ spacing, 0 overlap)}

The second approach for determining model order entails tracking the wavenumbers as the model order is increased. This method assumes that after a certain order is achieved, all of the propagating modes will be found and further increases in order will yield only arbitrary poles rather than valid modes. Figure 3.3.14 illustrates the point; the seven modes found by PRAWNS are plotted for varying model order. The two approaches are in agreement in this example. Both recommend specification of a model order higher than 25 (actually, 23 in the wavenumber scheme). The close agreement supports the use of either technique; in the Nantucket case, the wavenumber approach worked much better. 


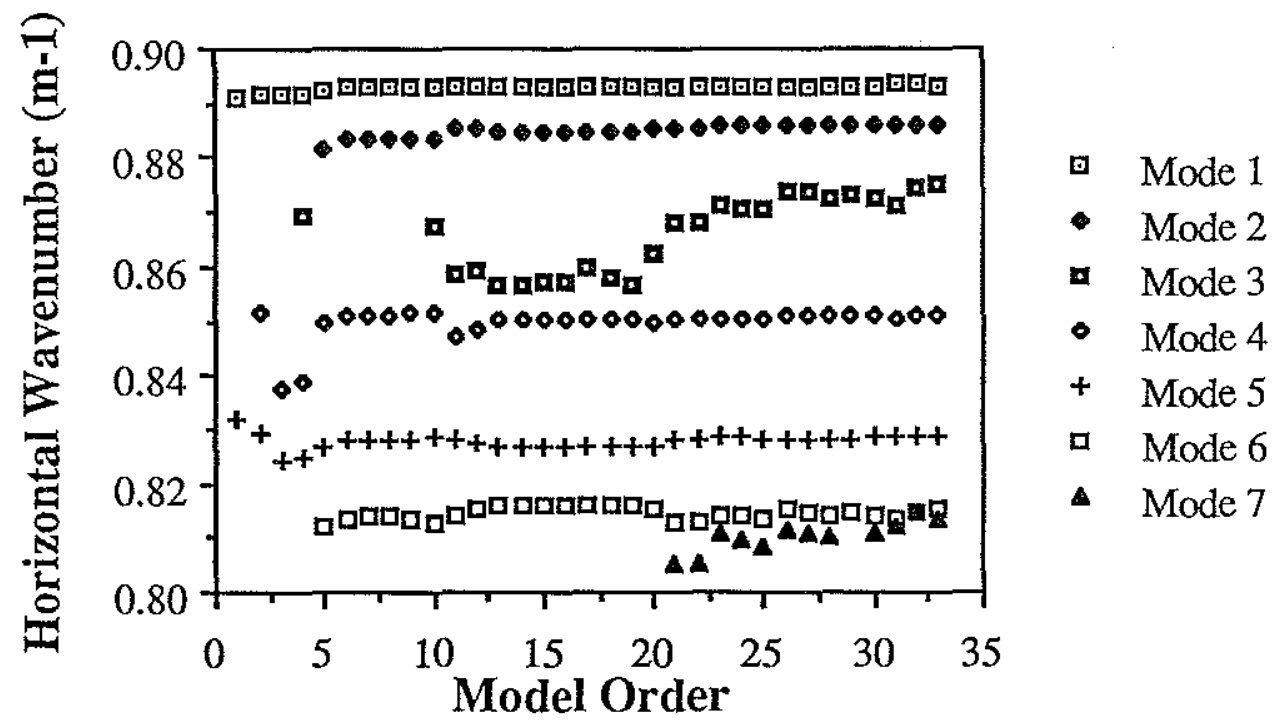

Fig 3.3.14 PRAWNS modes vs model order for Corpus Christi profile (100 pt, $3.4 \mathrm{~m}$ spacing, 0 overlap)

The graph also illustrates a shortcoming of the ESD plot. The ESD shows only six modes due to the damping effect (which controls the width of the ESD plot peaks) and a weak seventh mode amplitude. The seven wavenumbers found by PRAWNS algorithm are contained in the ESD graph; this is not evident until the tabular results are compared to the Green's function (which shows seven modes).

The pole plots for varying orders (figure 3.3.15) illustrate the pole wander effect of specifying the model order less than the system order ( shown for order $=5$ ) or specifying a model order which is higher than the actual order but not high enough (order $=10$ ). The slight variations in the pole locations are best seen in an overlap situation as depicted in figure 3.3.16. Even though model order 10 appears acceptable, close examination shows aberrations with respect to higher model order pole locations. 


\section{Pole plot of model order 5}

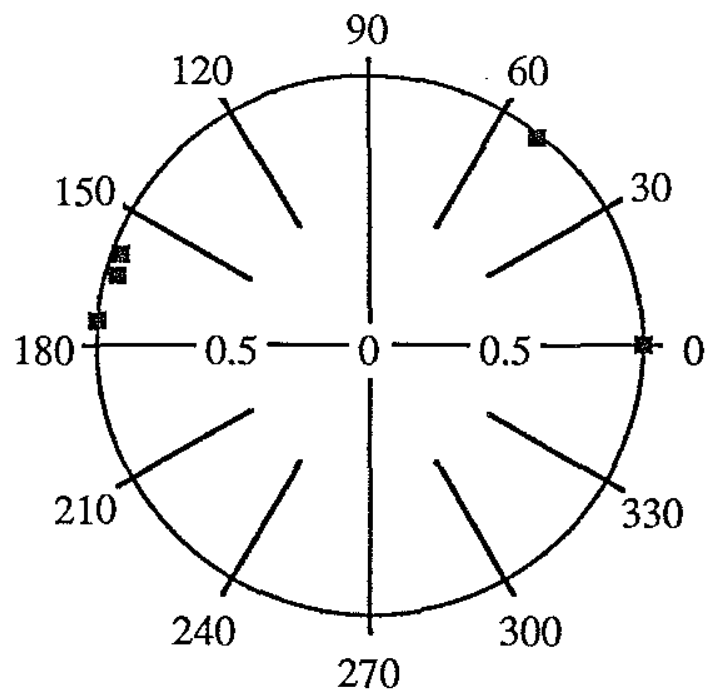

Pole plot for model order 10

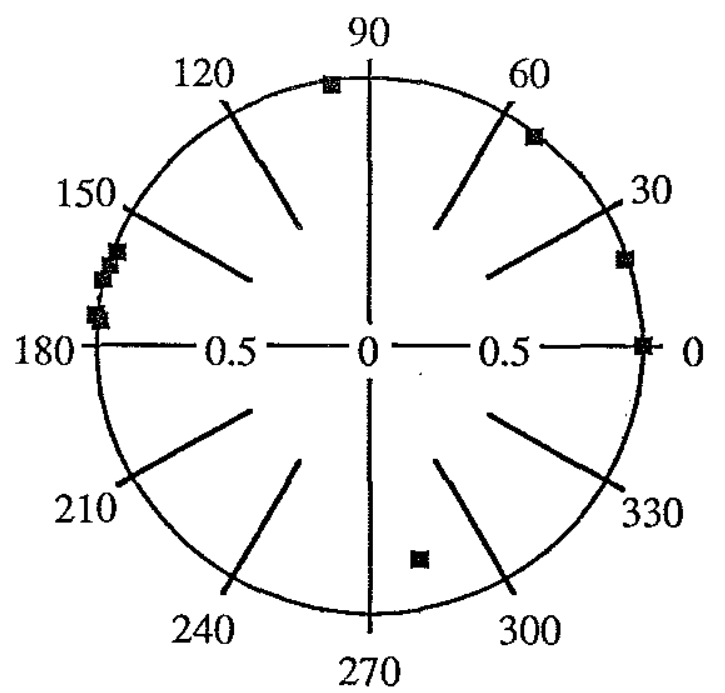

Fig 3.3.15 Pole plots of Corpus Christi data for varying model orders (100 pt, $3.4 \mathrm{~m}$ spacing, 0 overlap) 
Pole plot for model order 15

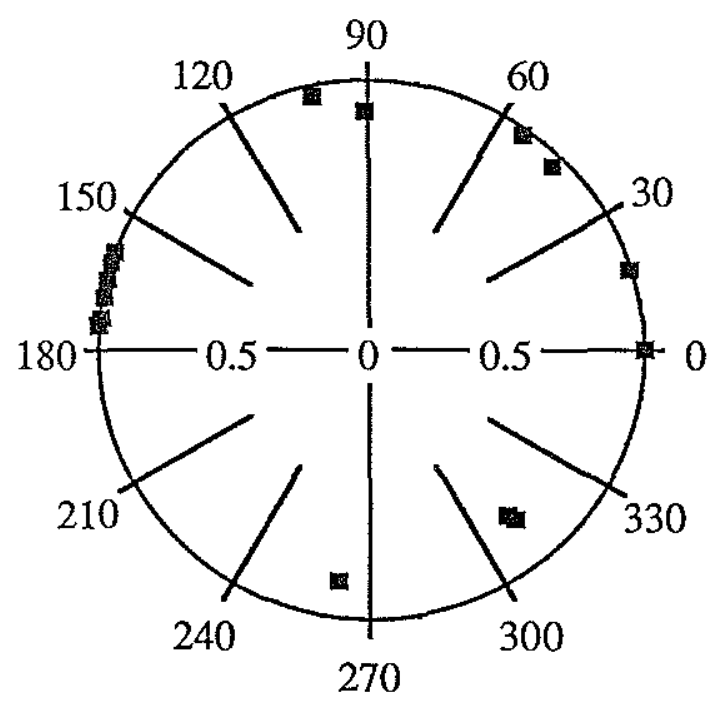

Pole plot for model order 35

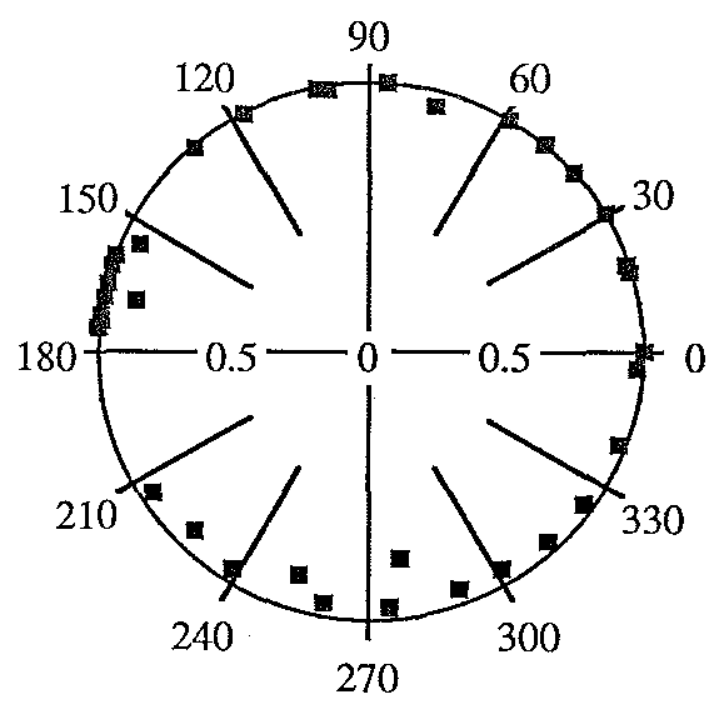

Fig 3.3.15(cont.) Pole plots of Corpus Christi data for varying model orders (100 pt, $3.4 \mathrm{~m}$ spacing, 0 overlap) 


\section{Comparision of model order 10 and 25}

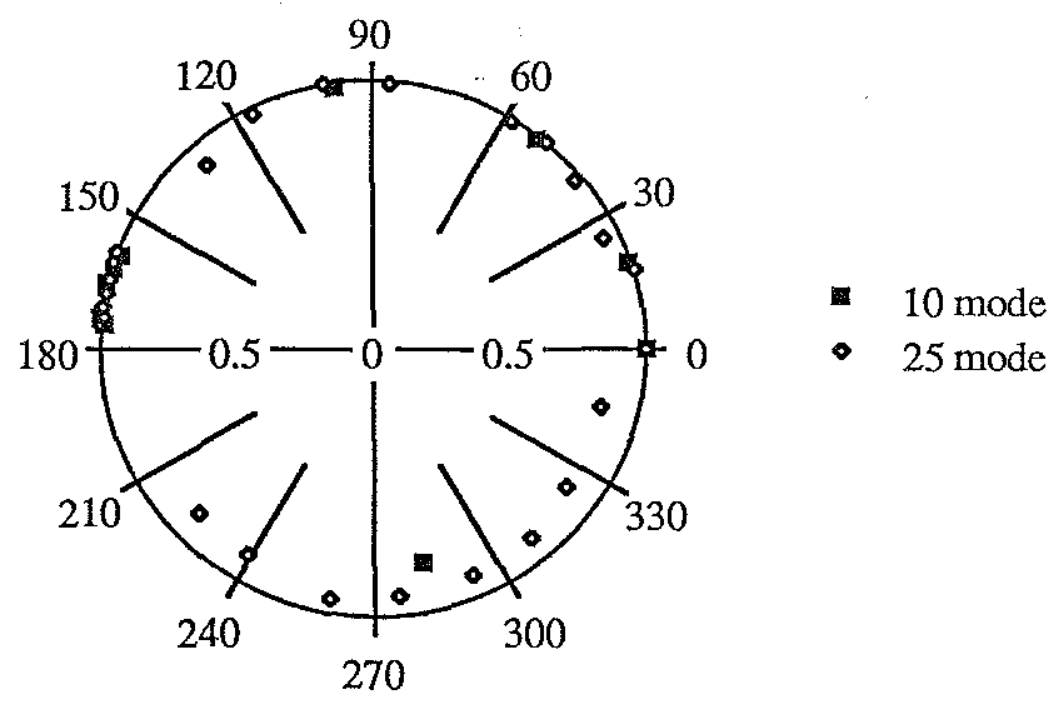

Fig 3.3.16 Overlay of model order 10 and 25 poles

In addition to model order selection, the effect of the amount of overlap on parameter estimation was examined. Overlap did not affect the resultant poles for actual modes. There was slight variation among the arbitrary poles with changes in overlap but there was no correlation. Figure 3.3.17 illustrates the invariance for the case of a 100 point aperture $(3.4 \mathrm{~m}$ spacing) 15 mode model. Figure 3.3.18 demonstrates the same behavior for a different aperture (200 points at $3.4 \mathrm{~m}$ spacing). 


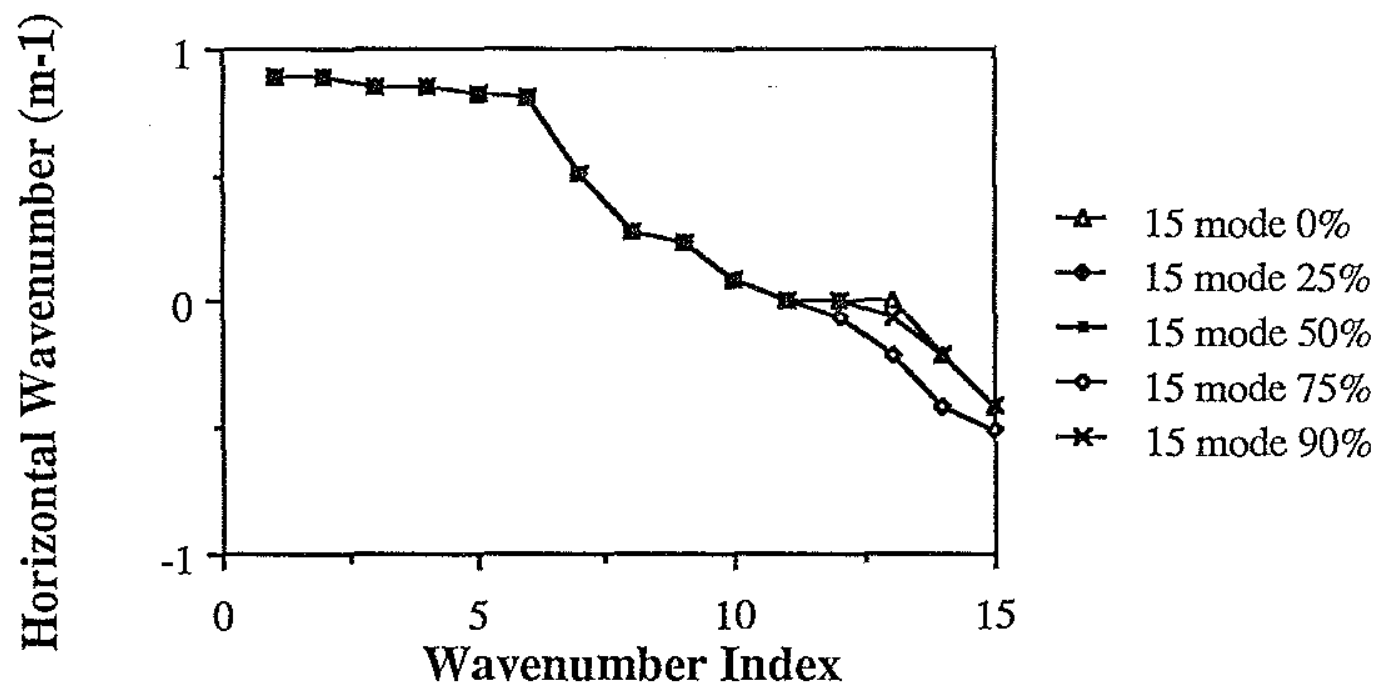

Fig 3.3.17 Overlap comparision of wavenumbers for model order 15 (Corpus Christi profile, 100 pt, $3.4 \mathrm{~m}$ spacing)

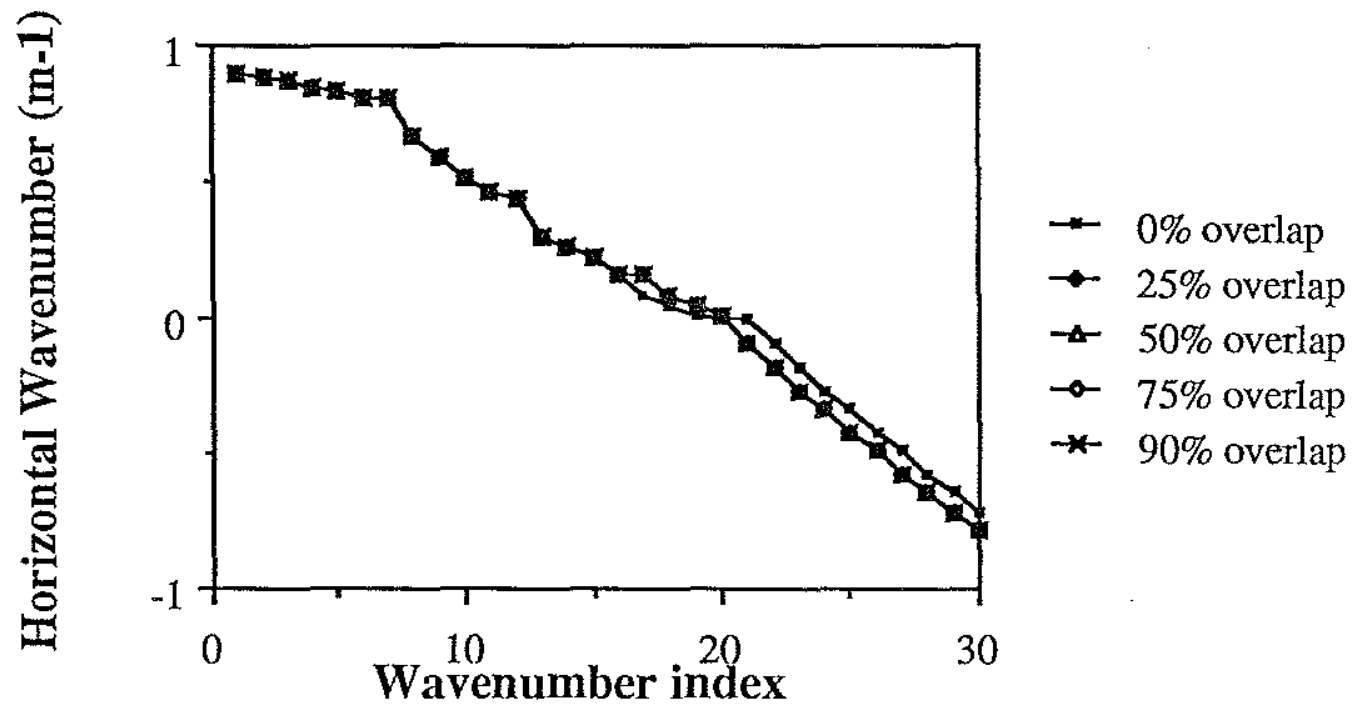

Fig 3.3.18 Overlap comparision of wavenumbers for model order 30 (Corpus Christi profile, 200 pt, $3.4 \mathrm{~m}$ spacing)

The arbitrary poles of the system varied with aperture size; indeed, the variation among these poles was even greater when model order and aperture were varied (figure 3.3.19). The 
wavenumber values for the propagating modes did not change since both the aperture and model order were large enough for an accurate estimation.

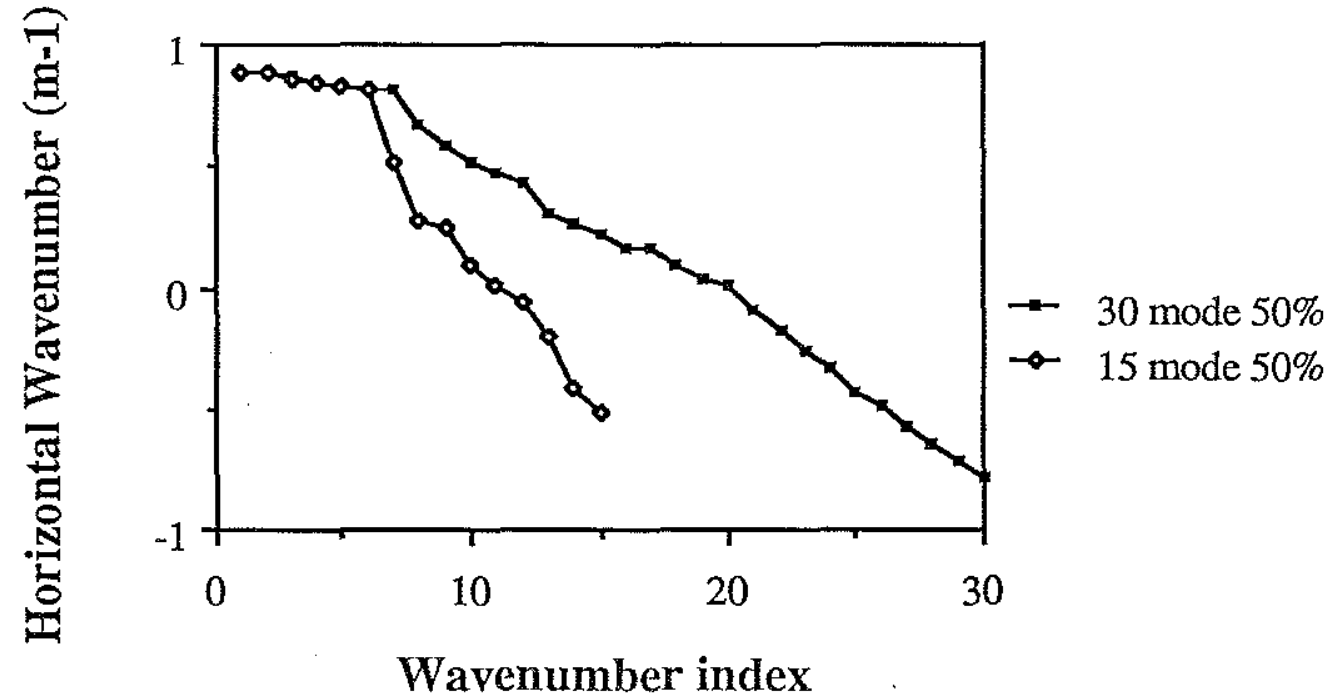

Fig 3.3.19 Comparision of 15 and 30 mode model wavenumbers (Corpus profile, 15 mode $\Rightarrow 100 \mathrm{pt}, 30$ mode $\Rightarrow 200 \mathrm{pt}, 3.4 \mathrm{~m}$ spacing)

Averaging had an interesting effect on the PRAWNS output. When averaging was used, the wavenumber variation was quite small. Recall that the averaging is not a frequency domain periodogram but rather an average of the discrete parameter components according to wavenumber. This process had little effect on the wavenumbers as illustrated in figure 3.3.20. 


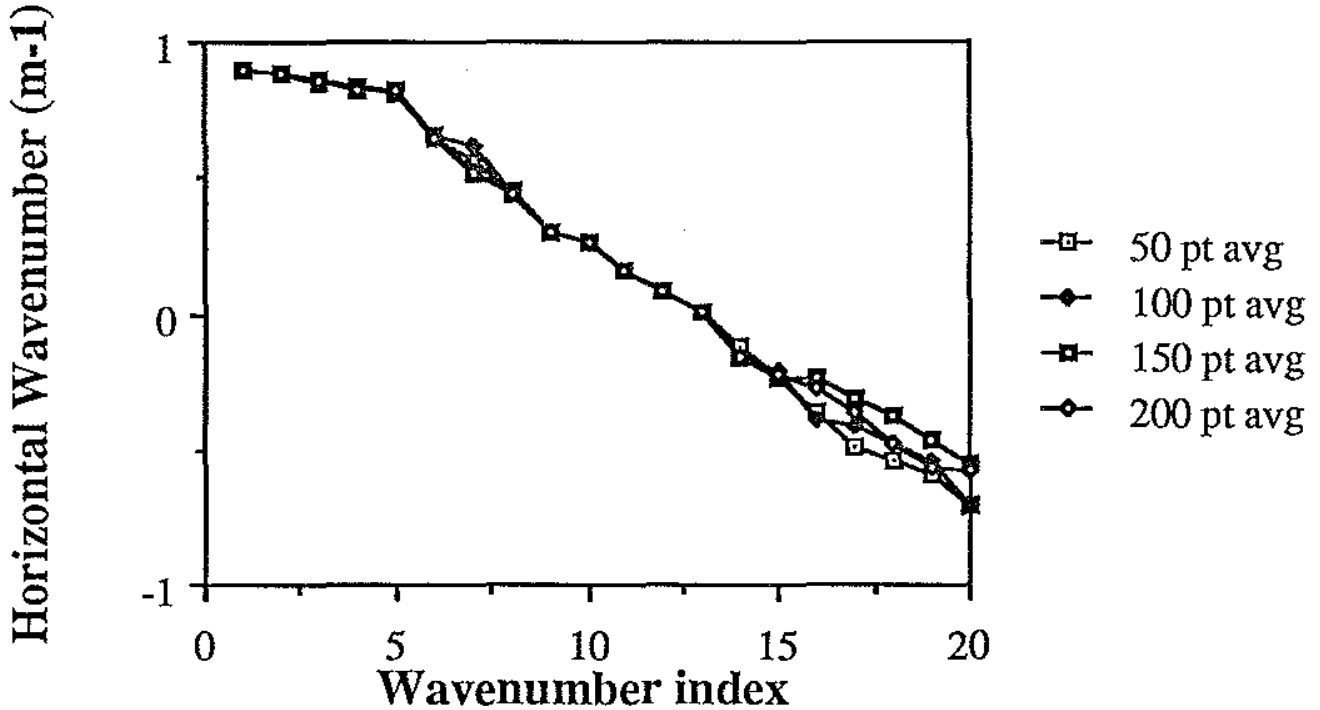

Fig 3.3.20 Wavenumber comparision for various averaging blocks ( Corpus Christi, 50 point proc. block, 0 overlap, model order 20)

Figure 3.3.21 illustrates the drop in total residue as the number of processing blocks averaged increases. The better fit to an all pole model may result in the averaging scheme being used as an effective tool in a noisy environment.

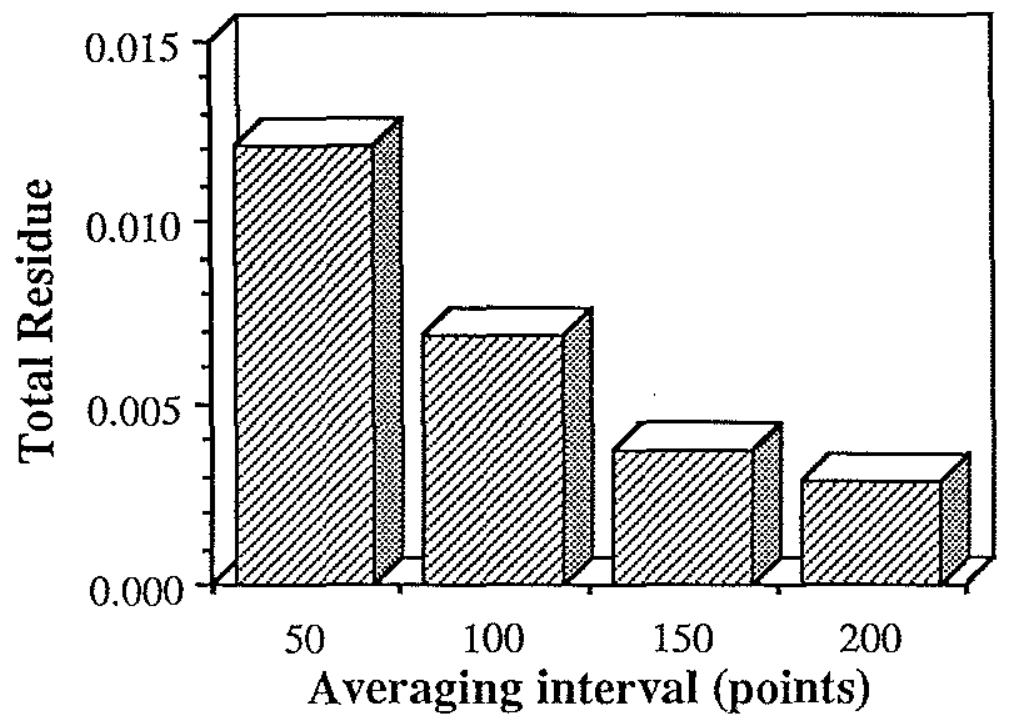

Fig 3.3.21 Wavenumber comparision for various averaging blocks (Corpus Christi, 50 point processing block, 0 overlap and model order 20) 


\subsection{Prony's Method in a Noisy Environment}

The feasibility of Prony's method with synthetically generated sound pressure fields has been demonstrated in the previous section. A major artificiality which has more effect than the approximations made in the synthetic pressure field generation algorithms is the absence of noise in the data. The experimental setup described in the first chapter is rife with opportunities for data corruption from measurement error, environmental sources and processing assumptions. This, then, is the ultimate test for the algorithm; how well does it perform in the real world?

Historically, Prony's method is particularly vulnerable to noise[3,4,43,44]. Methods such as the covariance or autocorrelation approaches discussed in chapter 2 for the first step of the process provide smoothing of data. The signal matrix approach used here does not provide any filtering except through the interpolation scheme used to obtain an evenly spaced set of data points. As a quantitative measure of the effects of noise, a Gaussian normal distribution routine (adapted from IMSL routine GGNML) was used[57]. The synthetic pressure field (generated by SAFARI) was corrupted with user set levels of noise. The effects oi the noise were easily compensated for until the SNR reached $30 \mathrm{~dB}$. The compensation took the form of larger apertures and use of overlap and averaging, and increases in model order. Overlapping was found effective up to approximately the $50 \%$ level; ie, each processing block overlapped the other by $50 \%$ and the results were averaged. At levels higher than $50 \%$, the additional overlap did not provide better results. The wavenumber degradation with respect to noise is shown in figures 3.4.1 through 3.4.4. In this particular example, compensation for the noise addition was through increases in model order. Note the better estimation of the higher order models at lower SNR. The other compensation used in conjunction with this was increasing the aperture size. Below a certain level, which was between 20 and $30 \mathrm{~dB}$, the compensation did not correct for the effects of noise. Other preprocessing techniques are necessary to obtain satisfactory results at the higher noise levels. It is interesting to note the 
degradation with respect to SNR is lowest for the lowest order modes although with the source-receiver geometry configuration of figure 2.3 .1 , at $220 \mathrm{~Hz}$, mode 2 has the strongest amplitude as seen in figure 3.3.3.

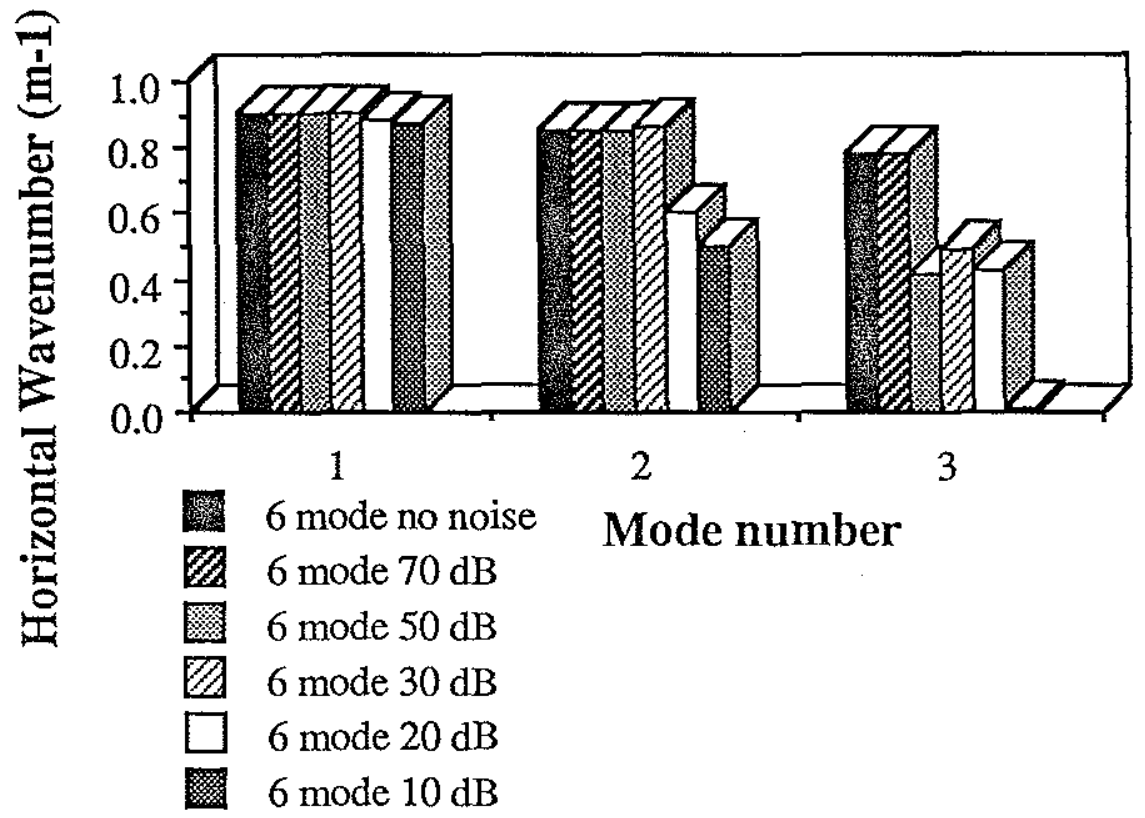

Fig 3.4.1 Wavenumber degradation for model order 6 of Nantucket profile (50 pt, $3.4 \mathrm{~m}$ spacing, model order 6, 50\% overlap) 


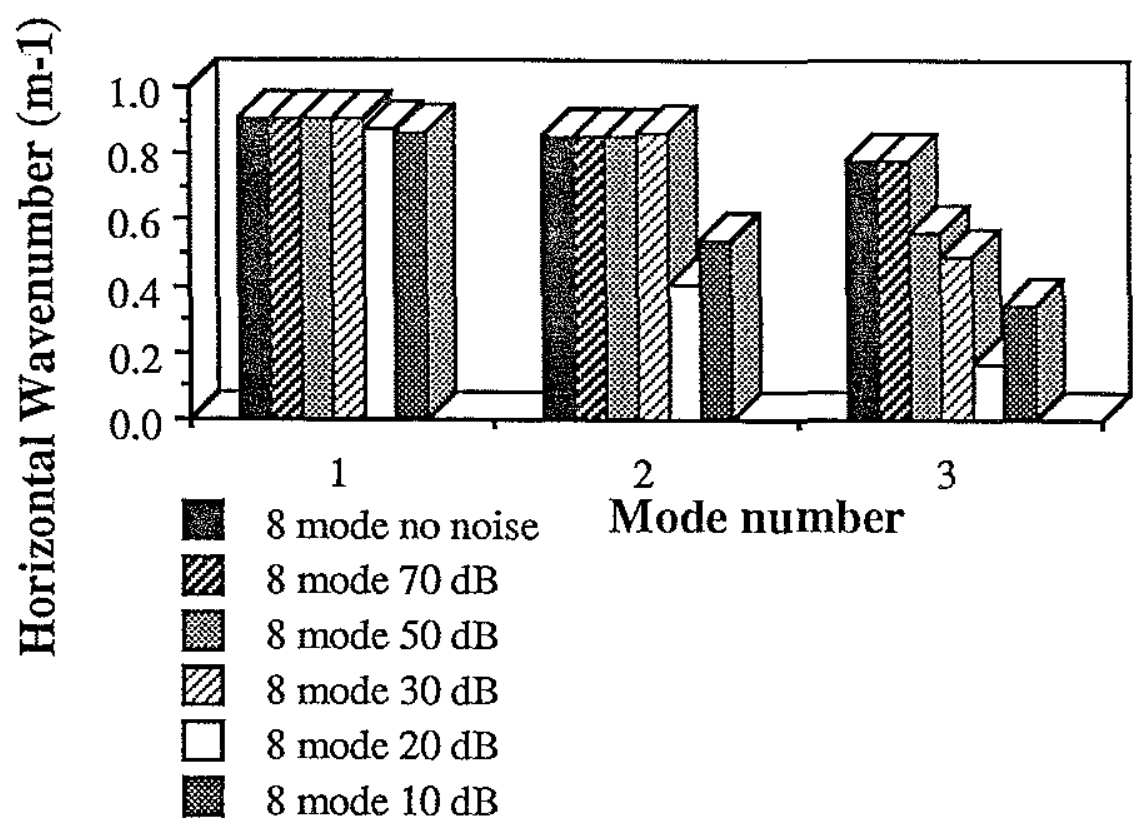

Fig 3.4.2 Wavenumber degradation for model order 8 of Nantucket profile (50 pt, $3.4 \mathrm{~m}$ spacing, model order $8,50 \%$ overlap)

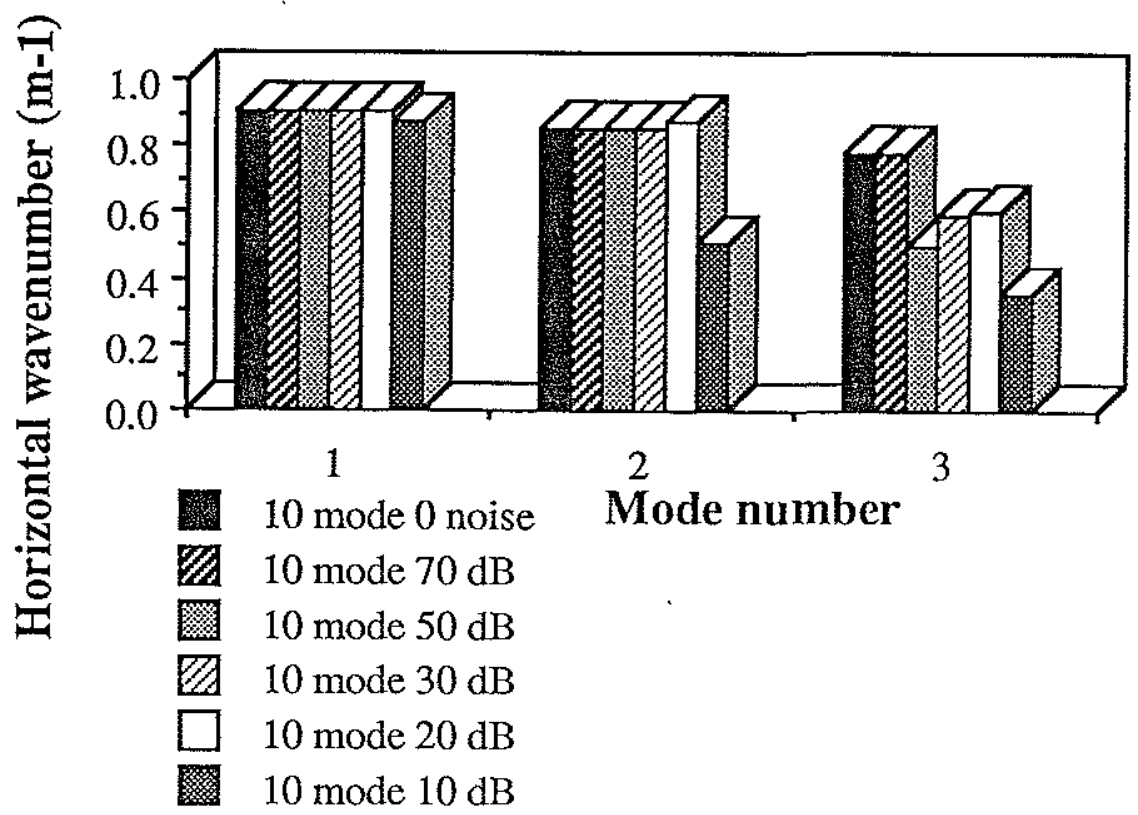

Fig 3.4.3 Wavenumber degradation for model order 10 of Nantucket profile $(50 \mathrm{pt}, 3.4 \mathrm{~m}$ spacing, model order $10,50 \%$ overlap) 


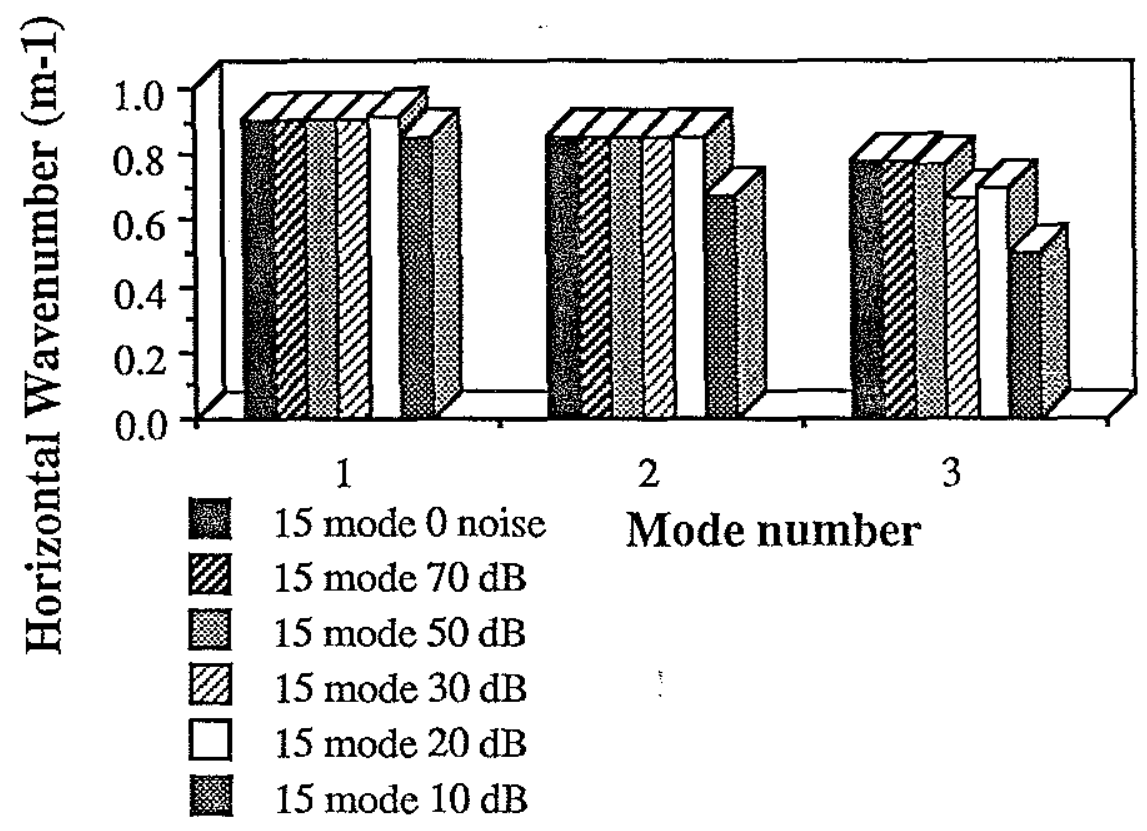

Fig 3.4.4 Wavenumber degradation for model order 15 of Nantucket profile (50 $\mathrm{pt}, 3.4 \mathrm{~m}$ spacing, model order $15,50 \%$ overlap)

Application of Prony's method to experimental data provides insight into the shortcomings of the present algorithm. The first set of field data was collected near Nantucket Island, MA in 1984. The bathymetry for the experiment is shown in figure 3.4.5. Note the depth change at approximately $600 \mathrm{~m}$. This change in waveguide dimensions should lead to a shift in modal peaks. Changes in the bottom sound speed profile will also affect pole values. 


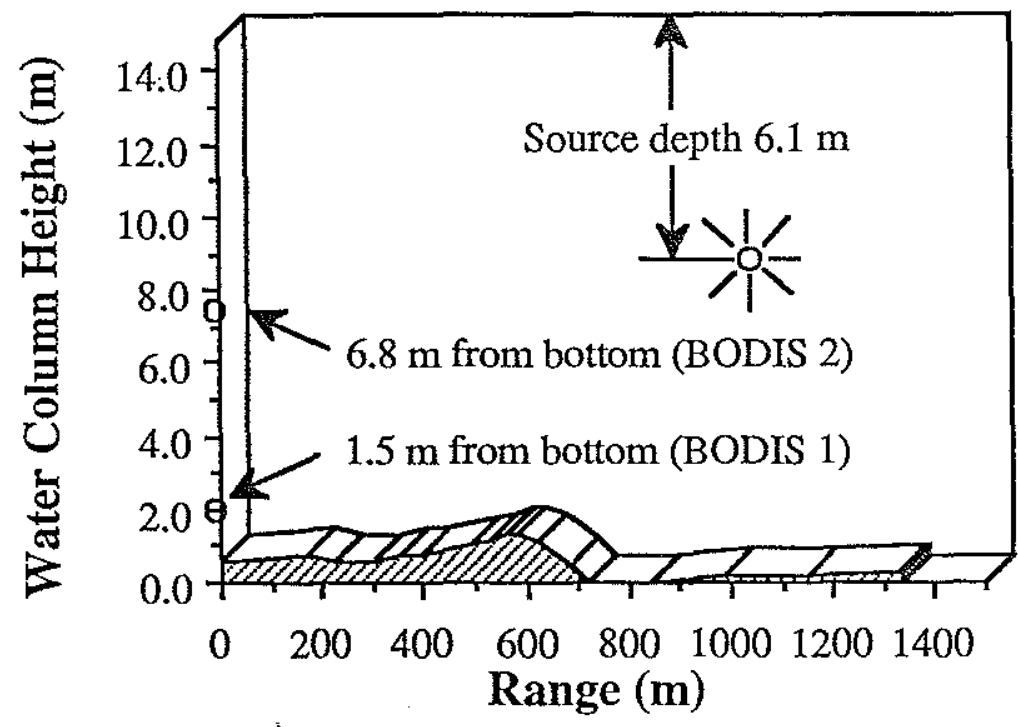

Fig 3.4.5 Nantucket bathymetry

The Green's function for this region is shown in figures 3.4.6 and 3.4.7 for the upper hydrophone at 140 and $220 \mathrm{~Hz}$ respectively. The Green's function was obtained by interpolating the data onto an evenly spaced grid and numerically Hankel transforming the entire data set. This assumes boundary condition invariance throughout the region of interest. From the bathymetry plot, it is obvious this condition is not met. The consequences of this is a spectrum with split modes (one set of modes before the bottom drop and one set after the drop) or smearing. 


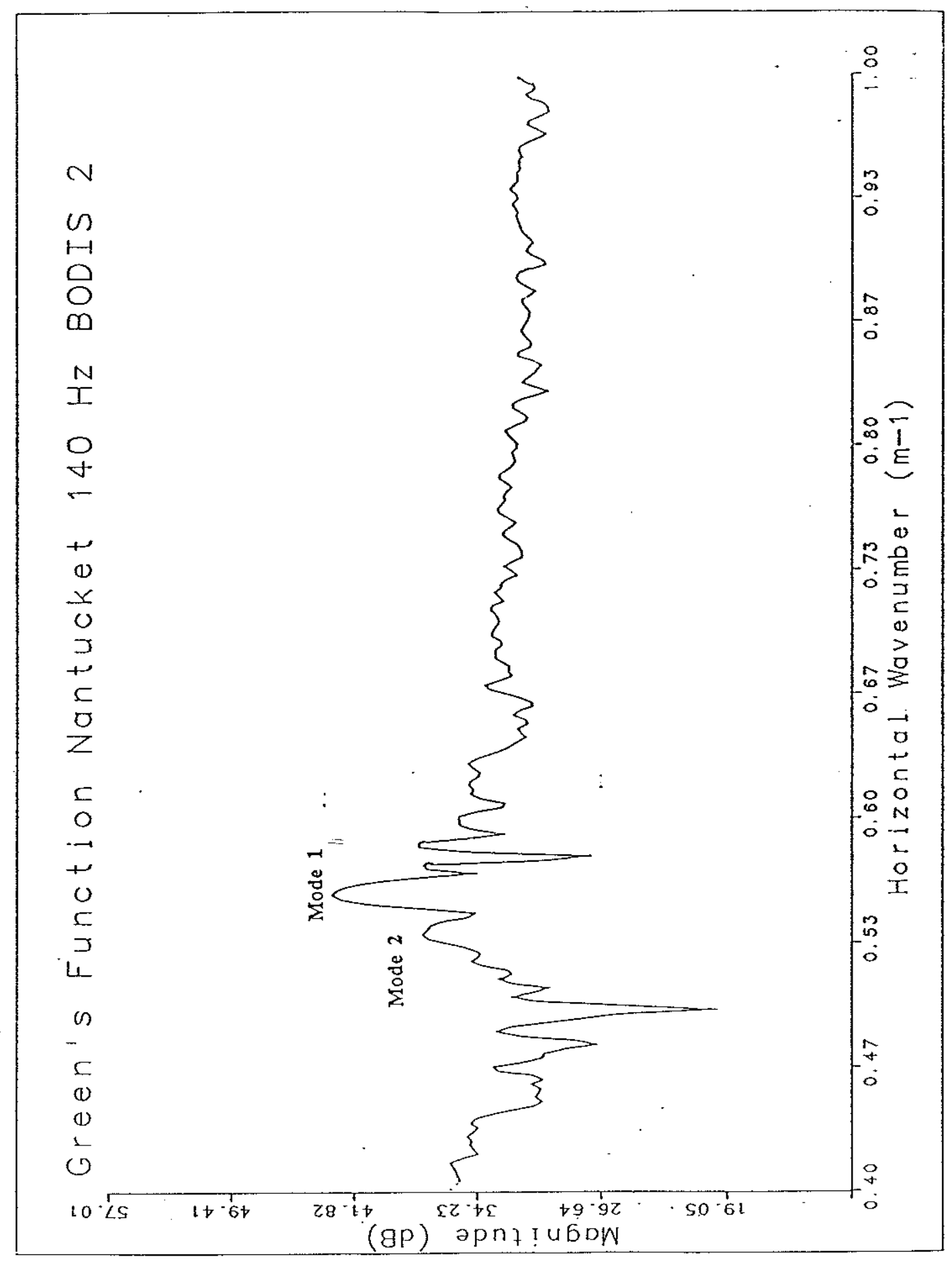

Fig 3.4.6 Green's function for Nantucket region (140 $\mathrm{Hz}$, Upper hydrophone) 


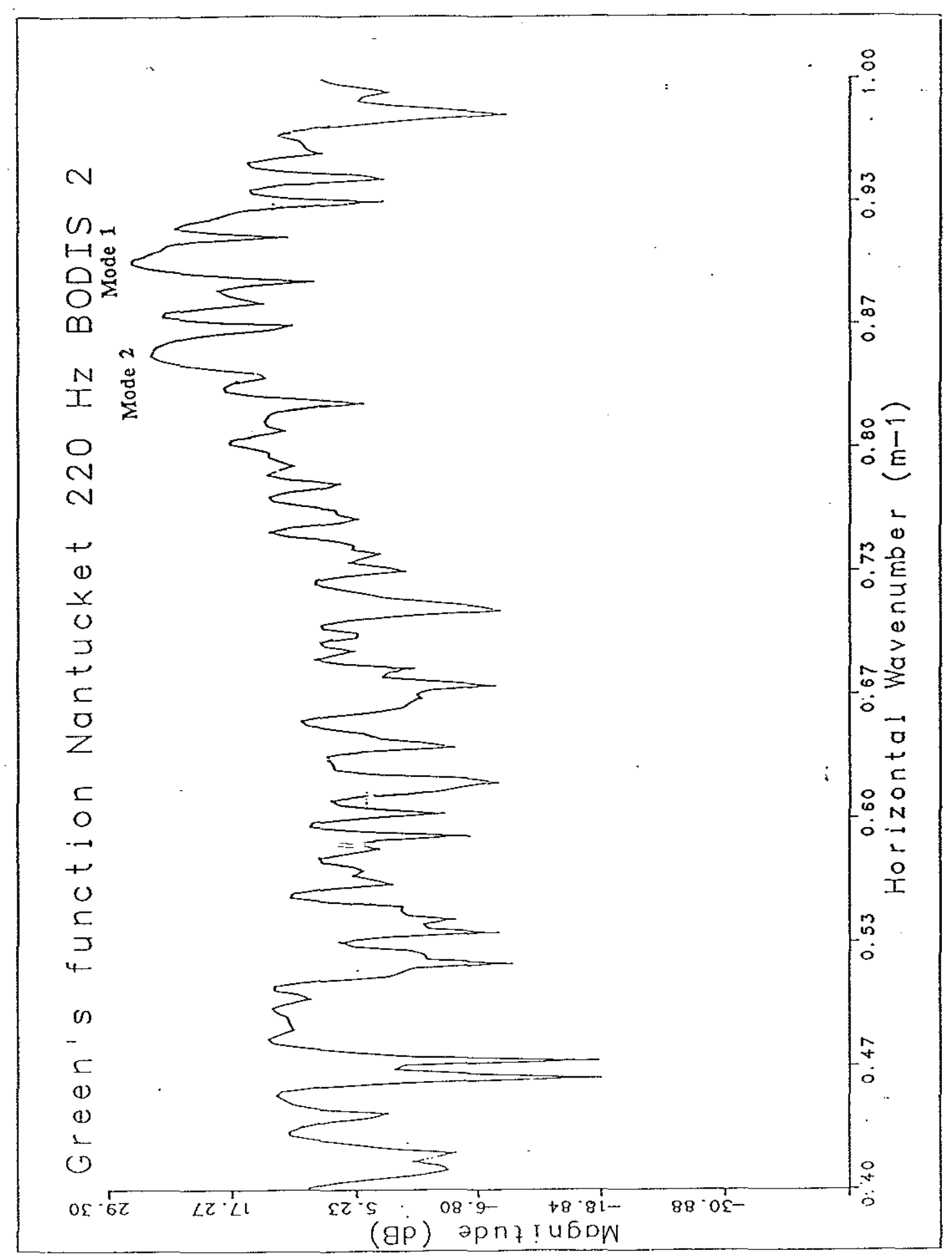

Fig 3.4.7 Green's function for Nantucket region (220 $\mathrm{Hz}$, Upper hydrophone) 
For Prony's method on the same data, the short aperture allows a much better approximation to range invariance. An aperture of 64 meters (100 points at $.64 \mathrm{~m}$ spacing) was chosen to exercise the algorithm for the $140 \mathrm{~Hz}$ and $220 \mathrm{~Hz}$ data sets. Since there is no "ground truth" for absolute comparision, the output of the PRAWNS code is best evaluated by comparing the two hydrophones for the same frequency. The different placement of the receivers changes the amount of energy in the modes but we expect the two ESDs to be similar. Figures 3.4.8 and 3.4.9 illustrate the good agreement for the $140 \mathrm{~Hz}$. case while figures 3.4.10 and 3.4.11 show the results of the $220 \mathrm{~Hz}$ case. The agreement between the corresponding hydrophones is obvious. 


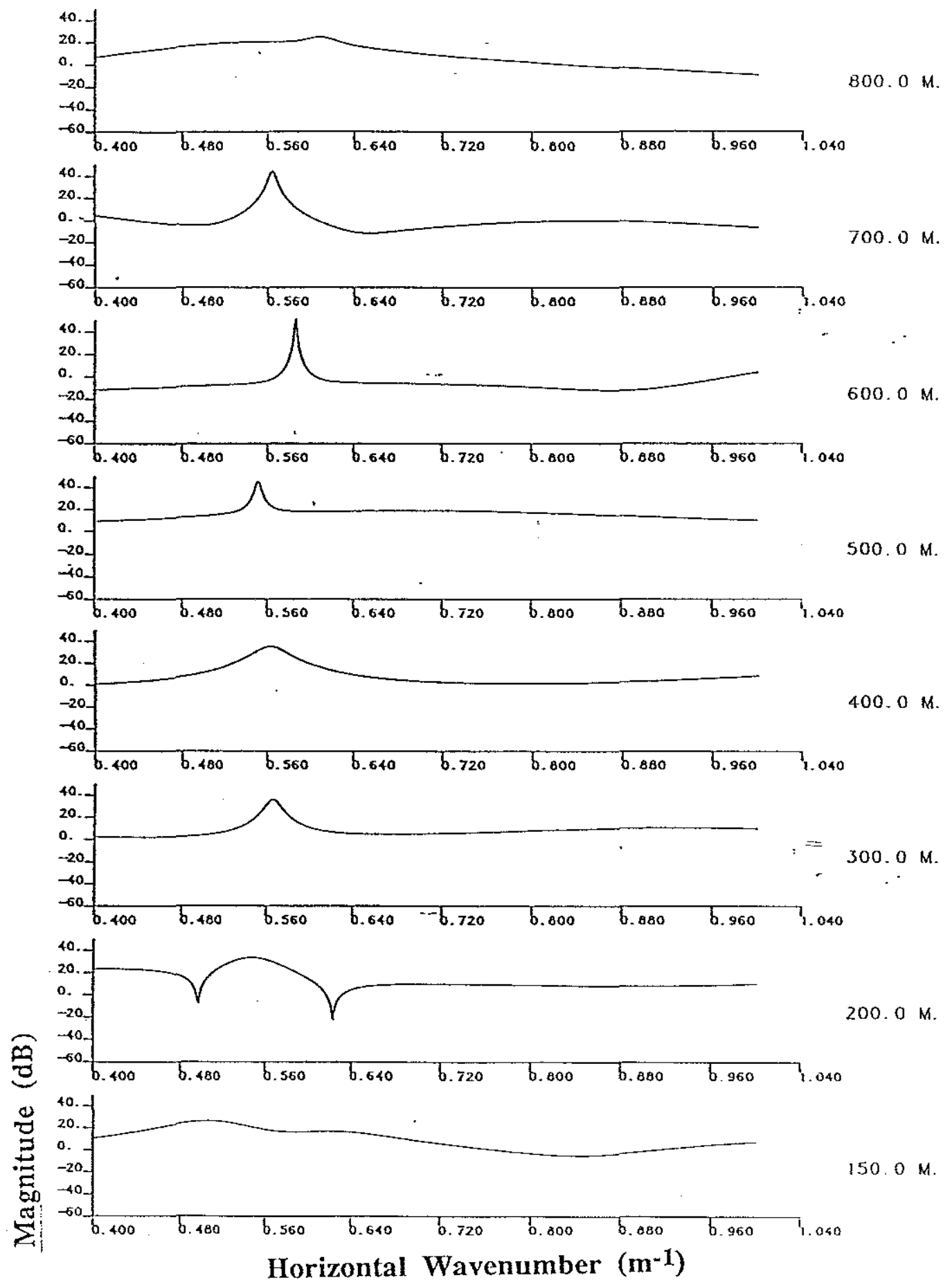

Fig 3.4.8 PRAWNS ESD for Nantucket $140 \mathrm{~Hz}$, upper hydrophone (100 pt, 50 pt overlap, $0.64 \mathrm{~m}$ spacing, 20 order mode) 


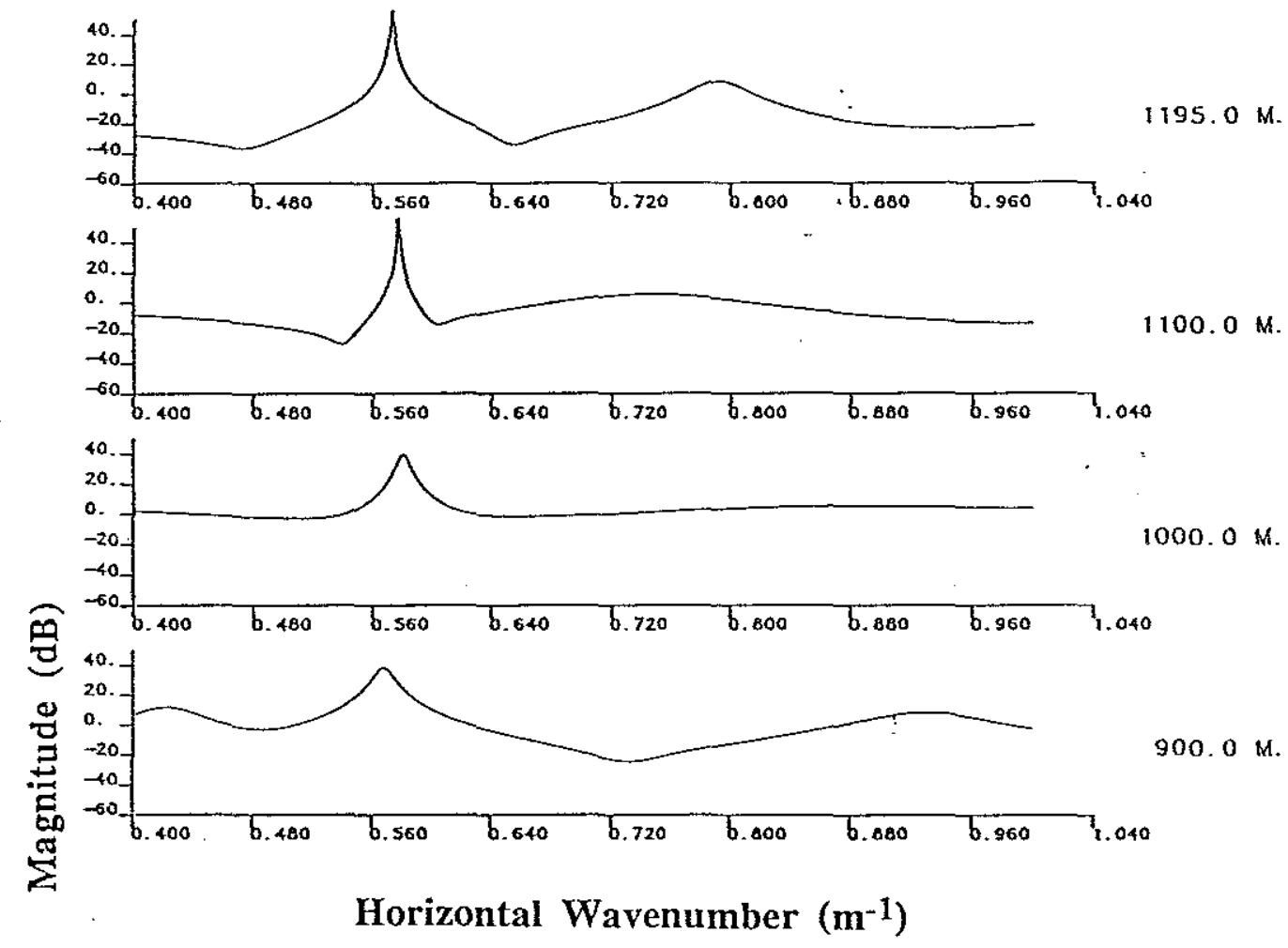

Fig 3.4.8(cont) PRAWNS ESD for Nantucket $140 \mathrm{~Hz}$, upper hydrophone (100 pt, 50 pt overlap, $0.64 \mathrm{~m}$ spacing, 20 order mode) 


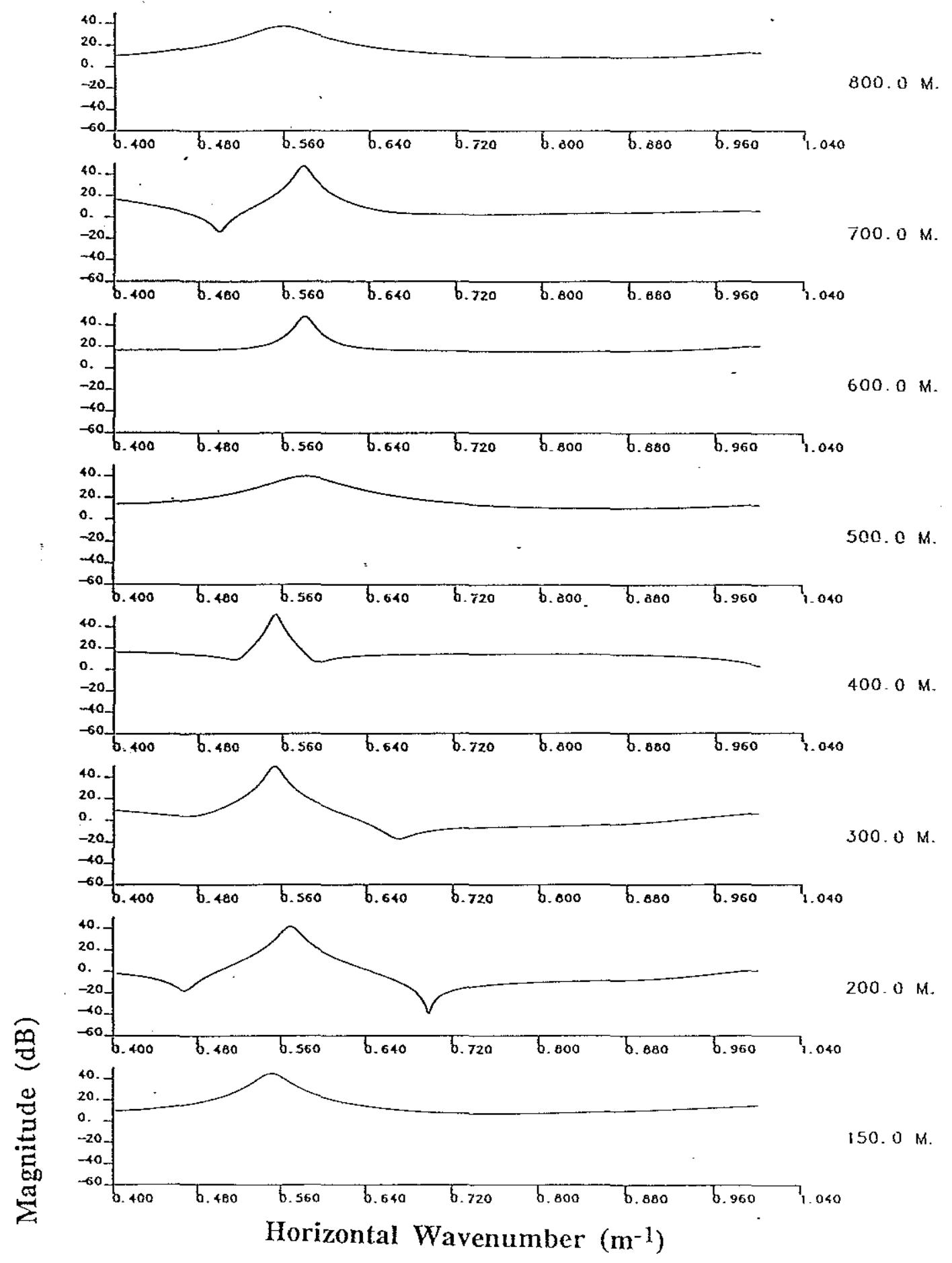

Fig 3.4.9 PRAWNS ESD for Nantucket $140 \mathrm{~Hz}$, lower hydrophone (100 pt, $50 \mathrm{pt}$ overlap, $0.64 \mathrm{~m}$ spacing, 20 order mode) 


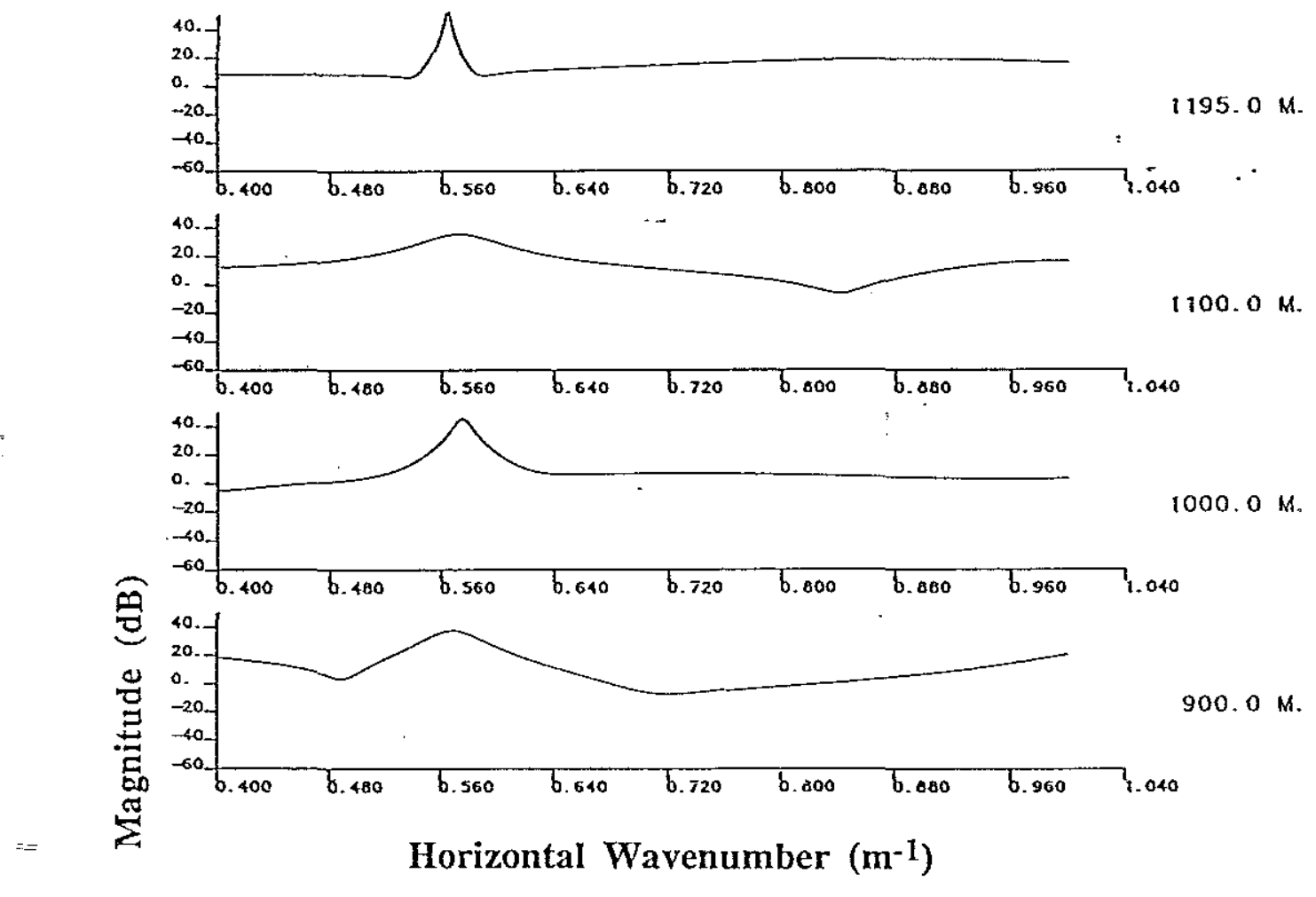

Fig 3.4.9(cont) PRAWNS ESD for Nantucket $140 \mathrm{~Hz}$, lower hydrophone (100 pt, $50 \mathrm{pt}$ overlap, $0.64 \mathrm{~m}$ spacing, 20 order mode) 


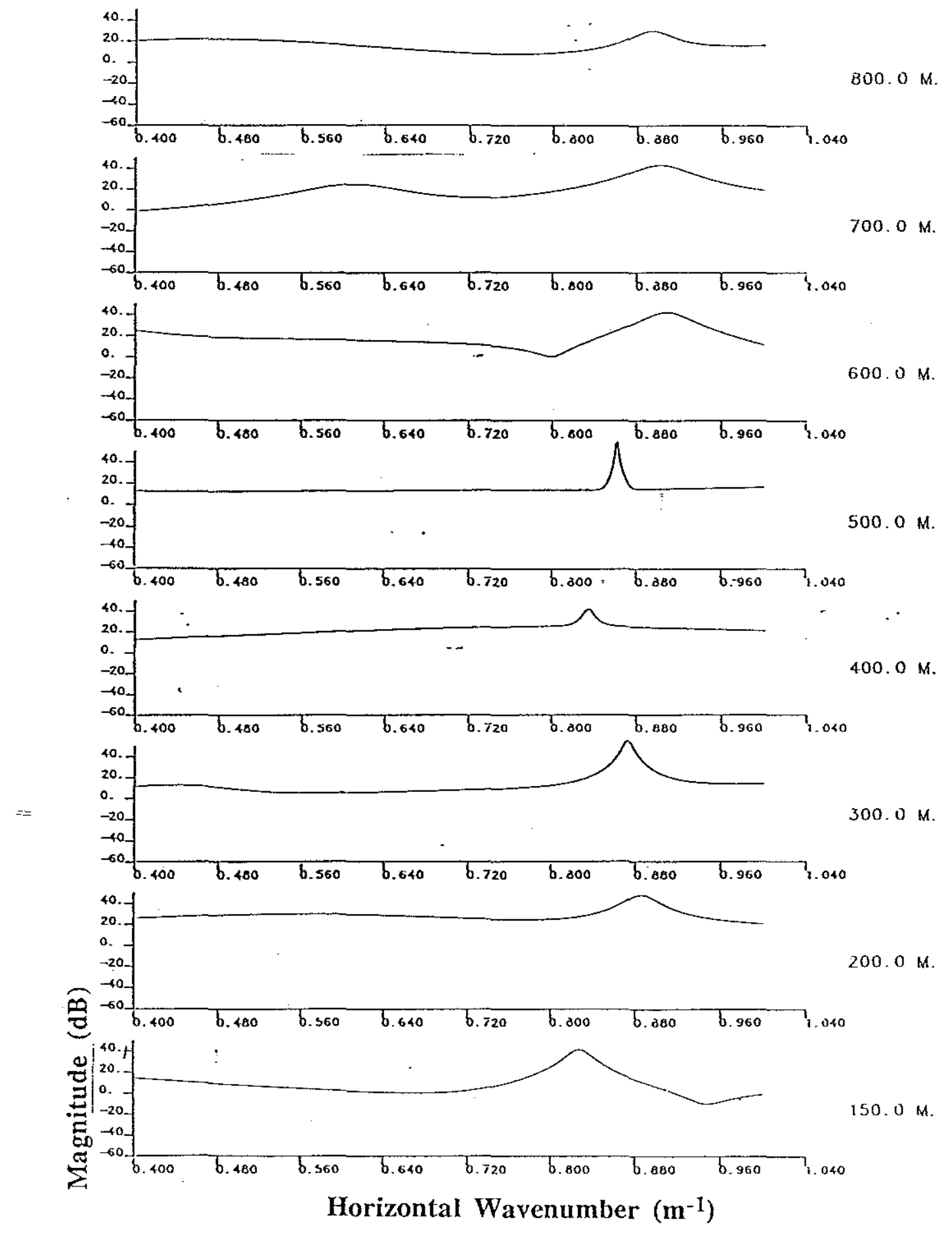

Fig 3.4.10 PRAWNS ESD for Nantucket $220 \mathrm{Hx}$, upper hydrophone (100 pt, 50 pt overlap, $0.64 \mathrm{~m}$ spacing, 20 order mode) 


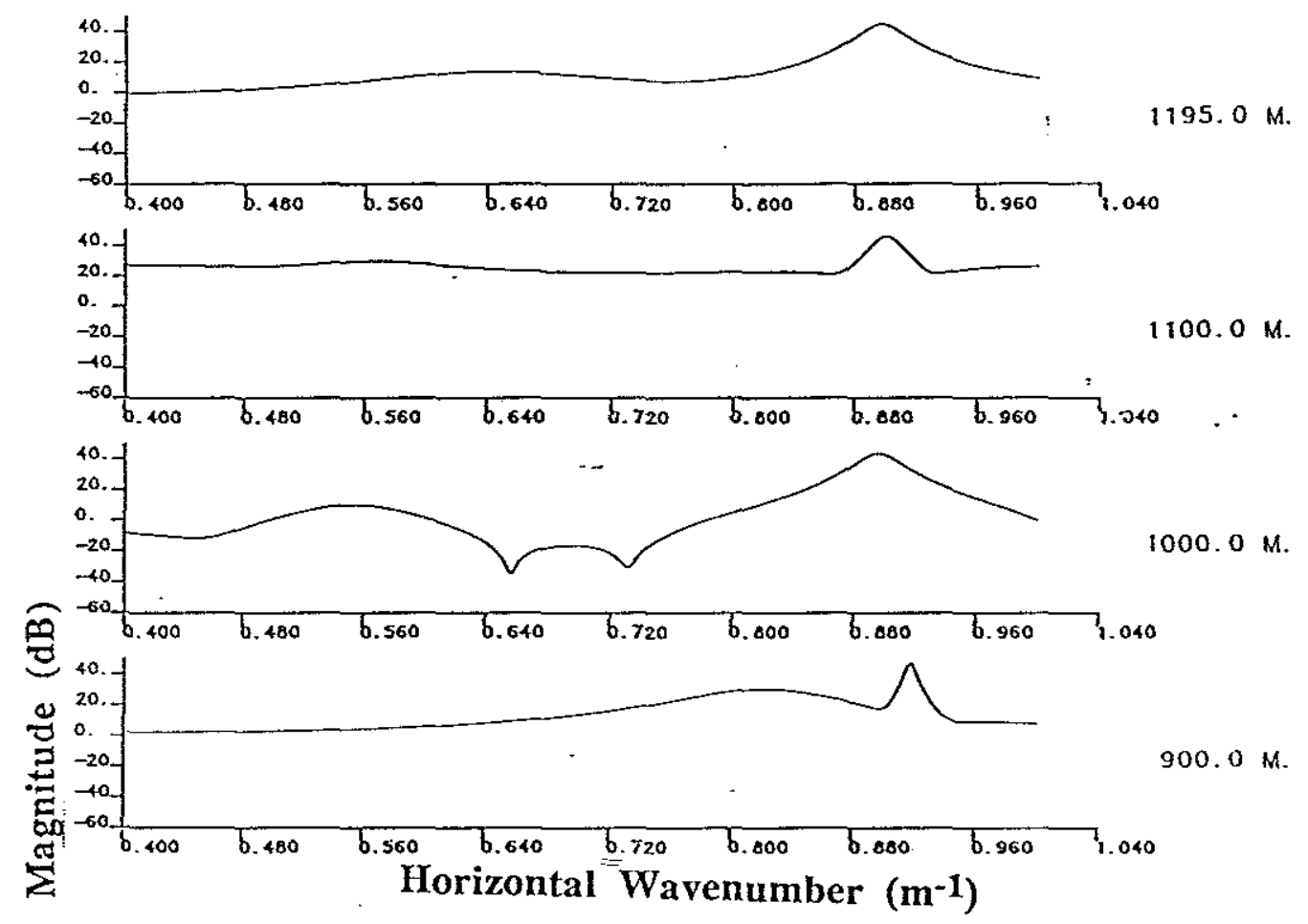

Fig 3.4.10(cont) PRAWNS ESD for Nantucket $220 \mathrm{~Hz}$, upper hydrophone (100 pt, 50 pt overlap, $0.64 \mathrm{~m}$ spacing, 20 order mode) 


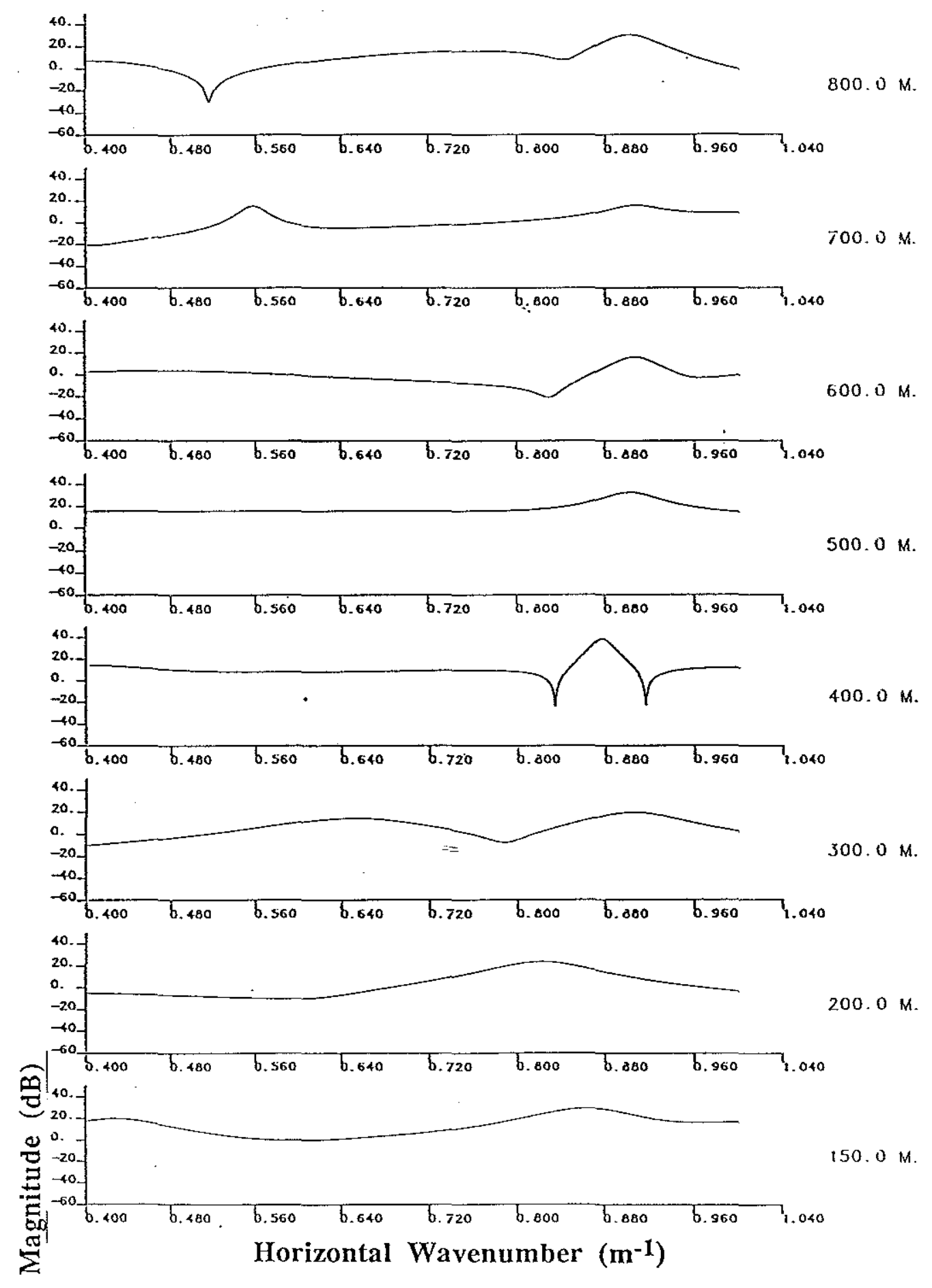

Fig 3.4.11 PRAWNS ESD for Nantucket $220 \mathrm{~Hz}$, lower hydrophone (100 pt, 50 pt overlap, $0.64 \mathrm{~m}$ spacing, 20 order mode) 


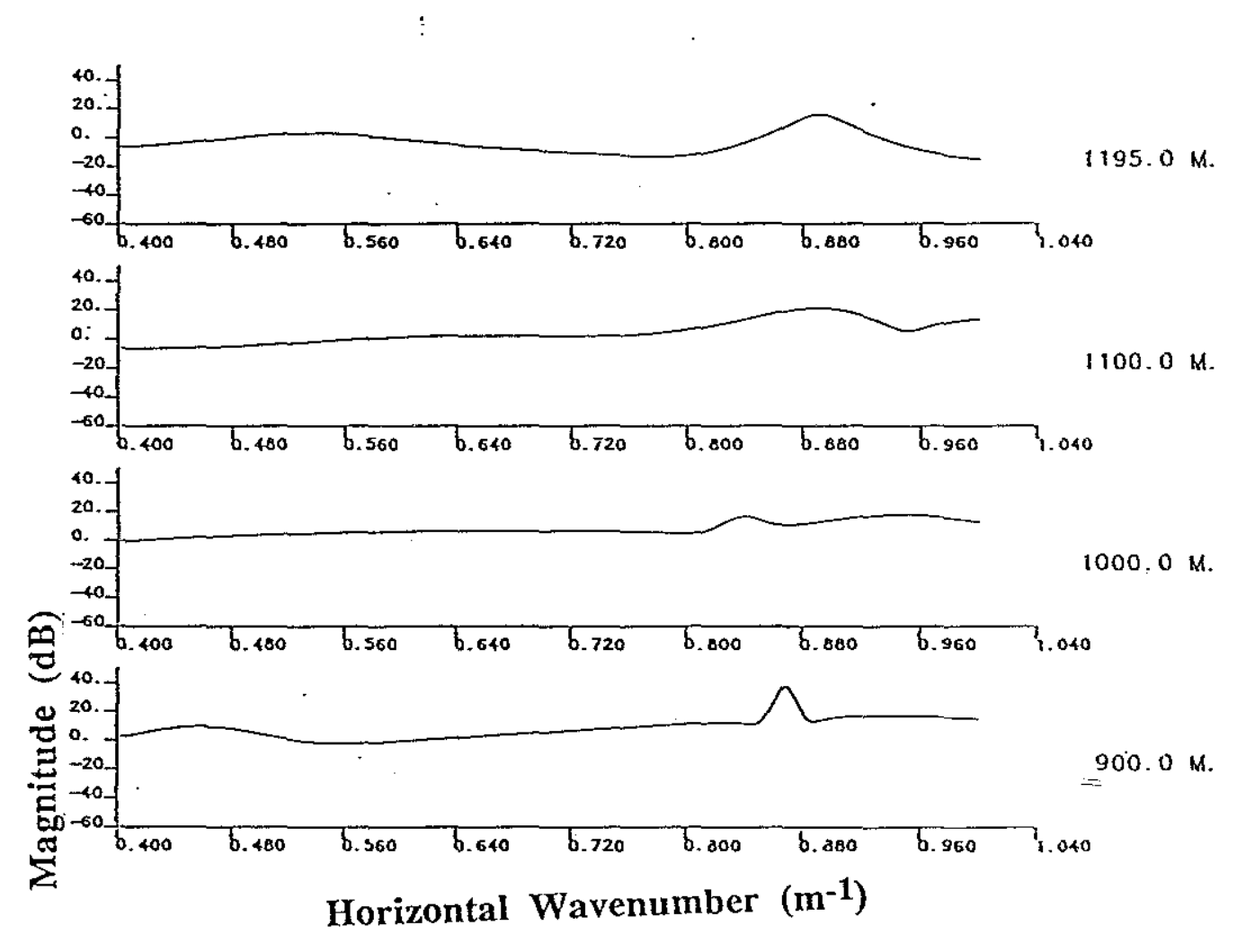

Fig 3.4.11(cont) PRAWNS ESD for Nantucket $220 \mathrm{~Hz}$, lower hydrophone (100 pt, $50 \mathrm{pt}$ overlap, $0.64 \mathrm{~m}$ spacing, 20 order mode) 
The Corpus Christi bathymetry is outlined in figure 3.4.12[24]. The frequencies used in this experiment were $50 \mathrm{~Hz}$ and $140 \mathrm{~Hz}$. The first hydrophone was moored $1.5 \mathrm{~m}$ from the bottom, the second was $30 \mathrm{~m}$ from the bottom. The water column depth at the receivers was $62.3 \mathrm{~m}$. The Green's function for each frequency is shown for the upper hydrophones (BODIS 2) in figures 3.4.13 and 3.4.14. Figures 3.4.15 through 3.4.18 illustrate the PRAWNS outputs for the experiment. The agreement between the hydrophones is again quite good.

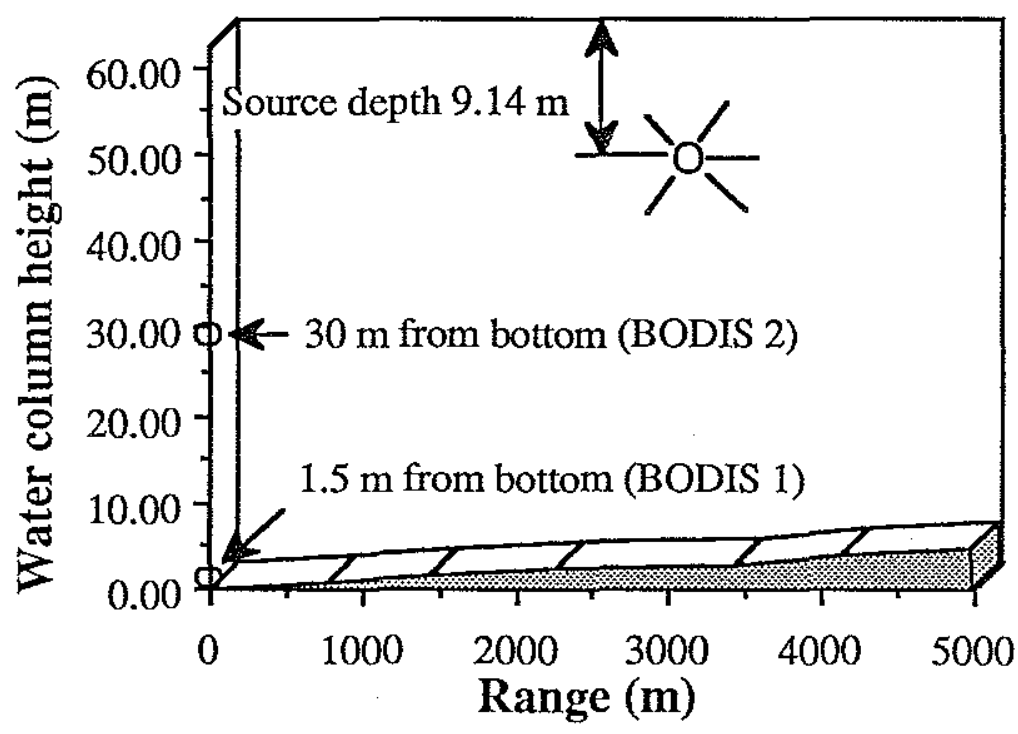

Fig 3.4.12 Corpus Christi bathymetry

The shortcomings of the present algorithm lie not in inconsistent results but rather in the lack of robust processing in noise. The modes are smeared together on the ESD plots. An additional preprocessing step is required to reduce the sensitivity of the method to noise. Chapter 5 contains one such proposed scheme for improved performance in noise. 


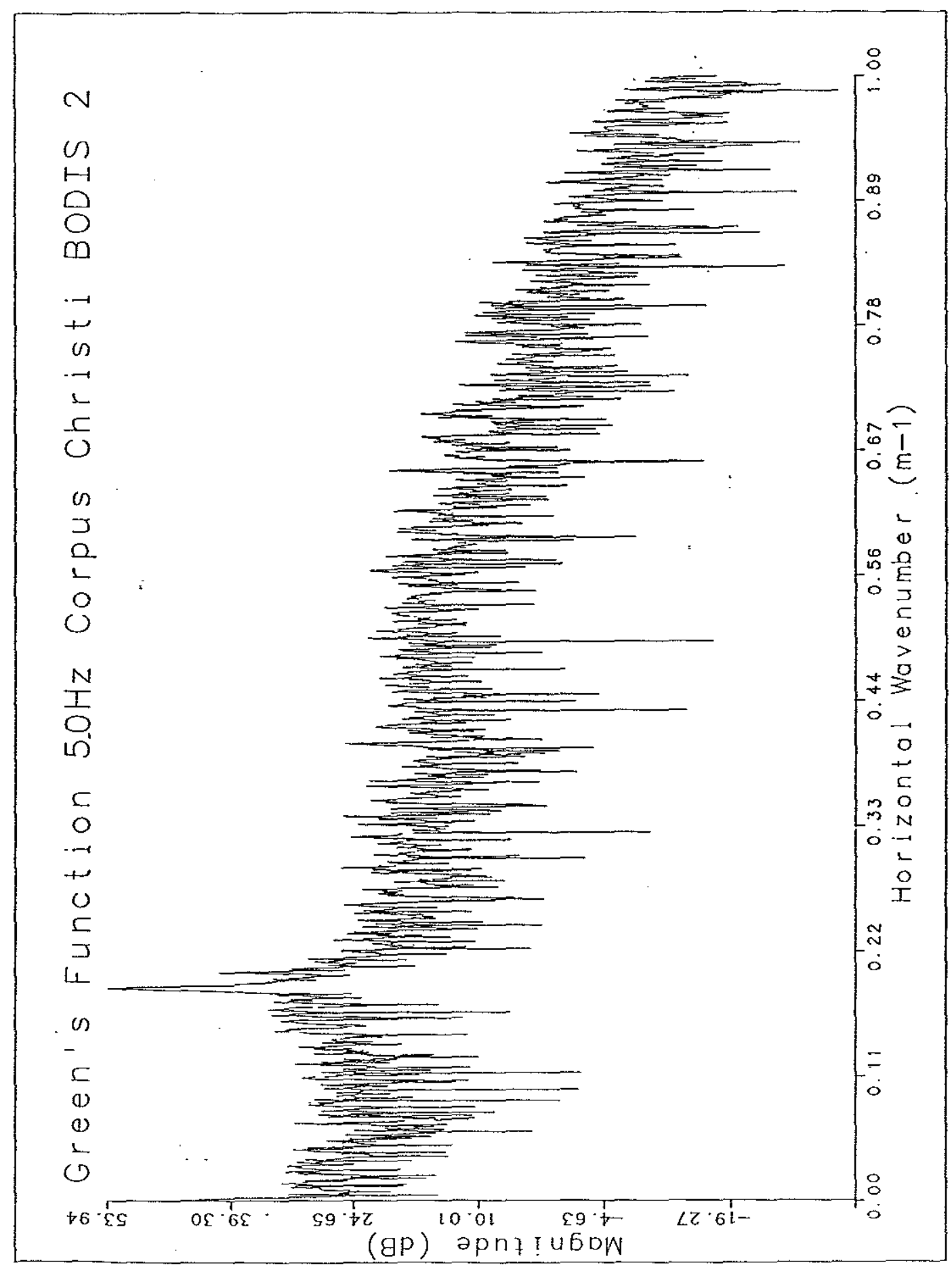

Fig 3.4.13 Green's function for Corpus Christi $50 \mathrm{~Hz}$, upper hydrophone 


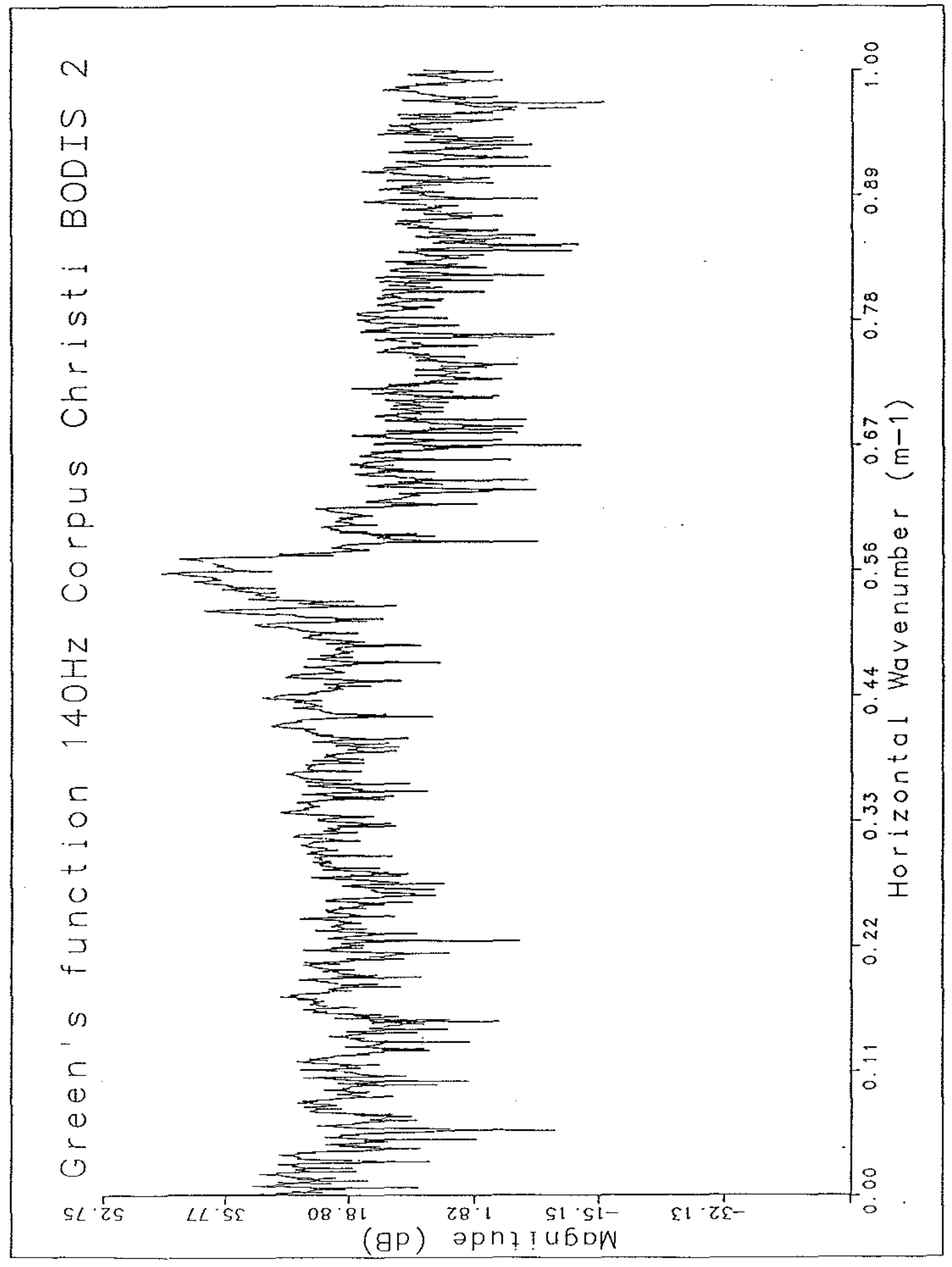

Fig 3.4.14 Green's function for Corpus Christi $140 \mathrm{~Hz}$, upper hydrophone $-92-$ 


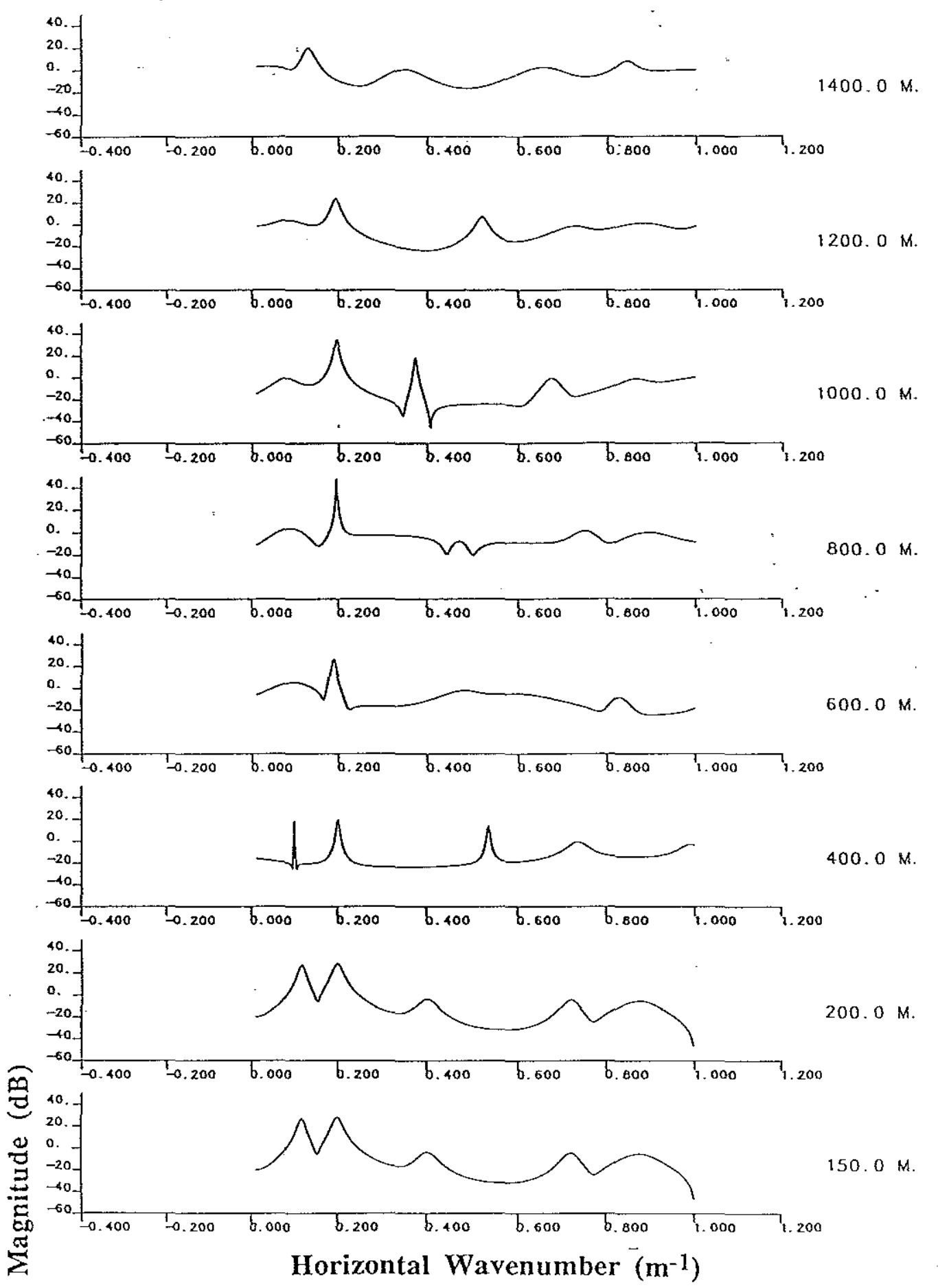

Fig 3.4.15 PRAWNS ESD for Corpus Christi $50 \mathrm{~Hz}$, upper hydrophone (100 pt, $50 \mathrm{pt}$ overlap, $1.23 \mathrm{~m}$ spacing, model order 25 ) 


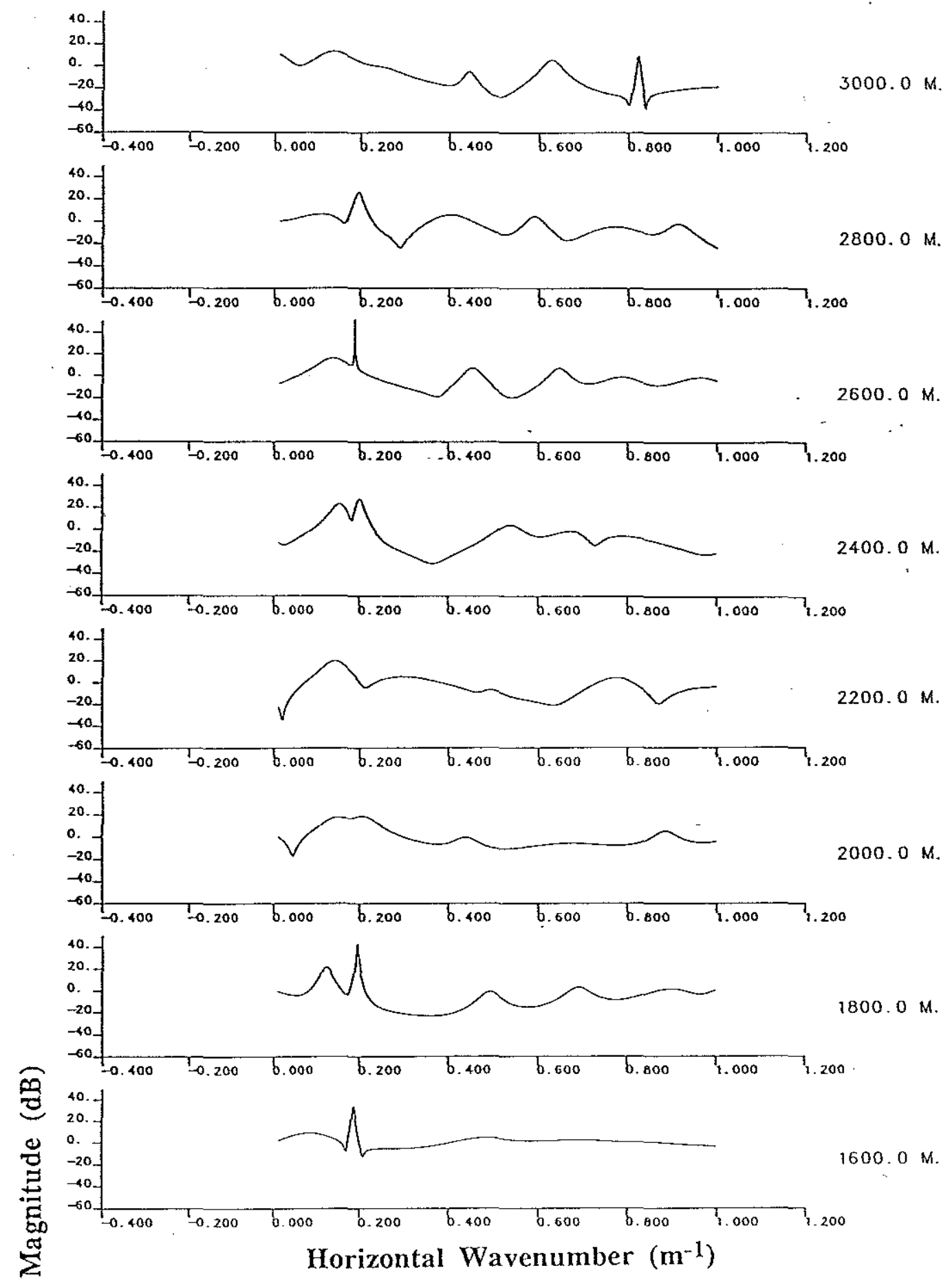

Fig 3.4.15(cont) PRAWNS ESD for Corpus Christi $50 \mathrm{~Hz}$, upper hydrophone $(100 \mathrm{pt}, 50 \mathrm{pt}$ overlap, $1.23 \mathrm{~m}$ spacing, model order 25) 


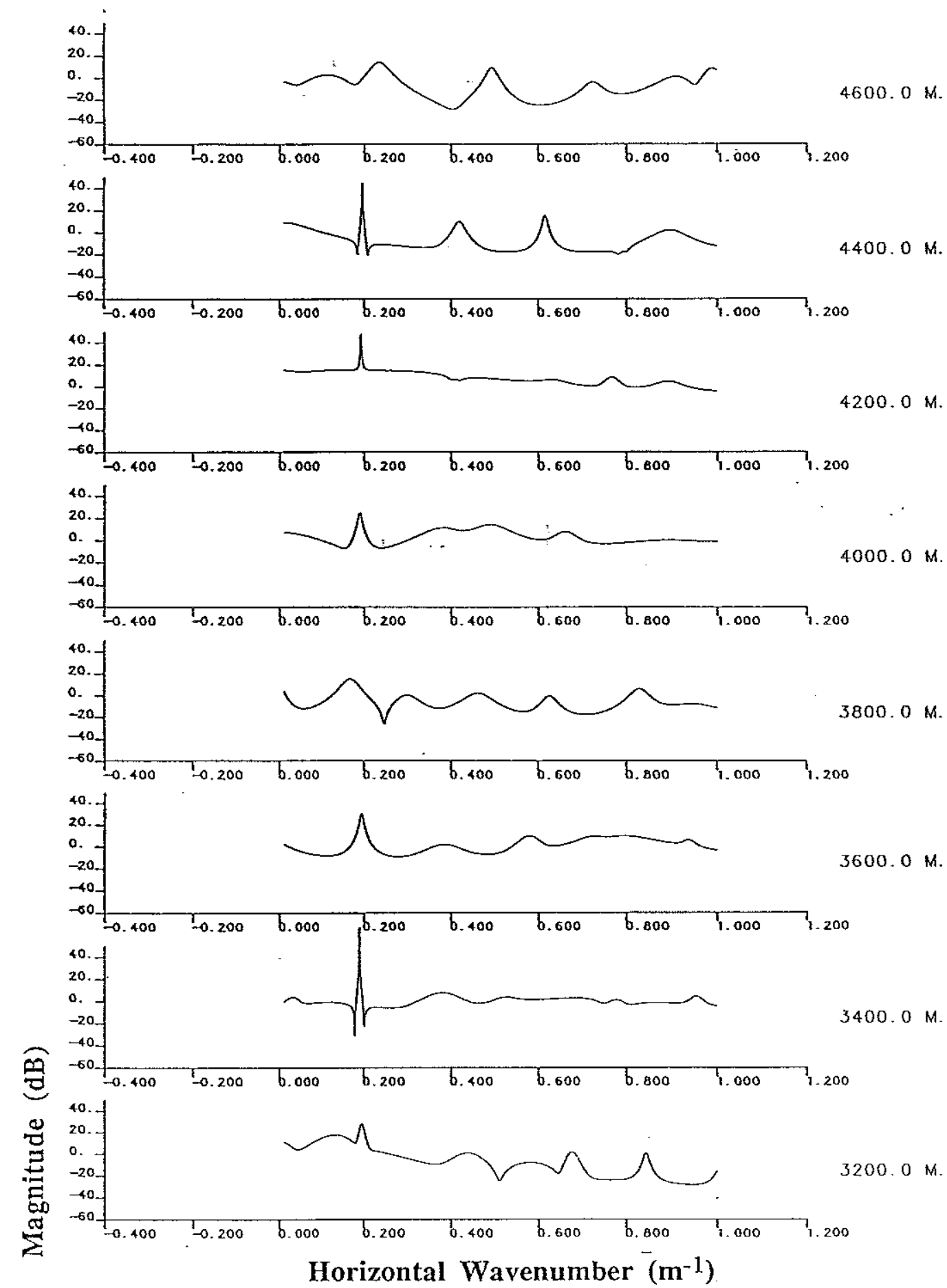

Fig 3.4.15(cont) PRAWNS ESD for Corpus Christi $50 \mathrm{~Hz}$, upper hydrophone (100 pt, 50 pt overlap, $1.23 \mathrm{~m}$ spacing, model order 25 ) 

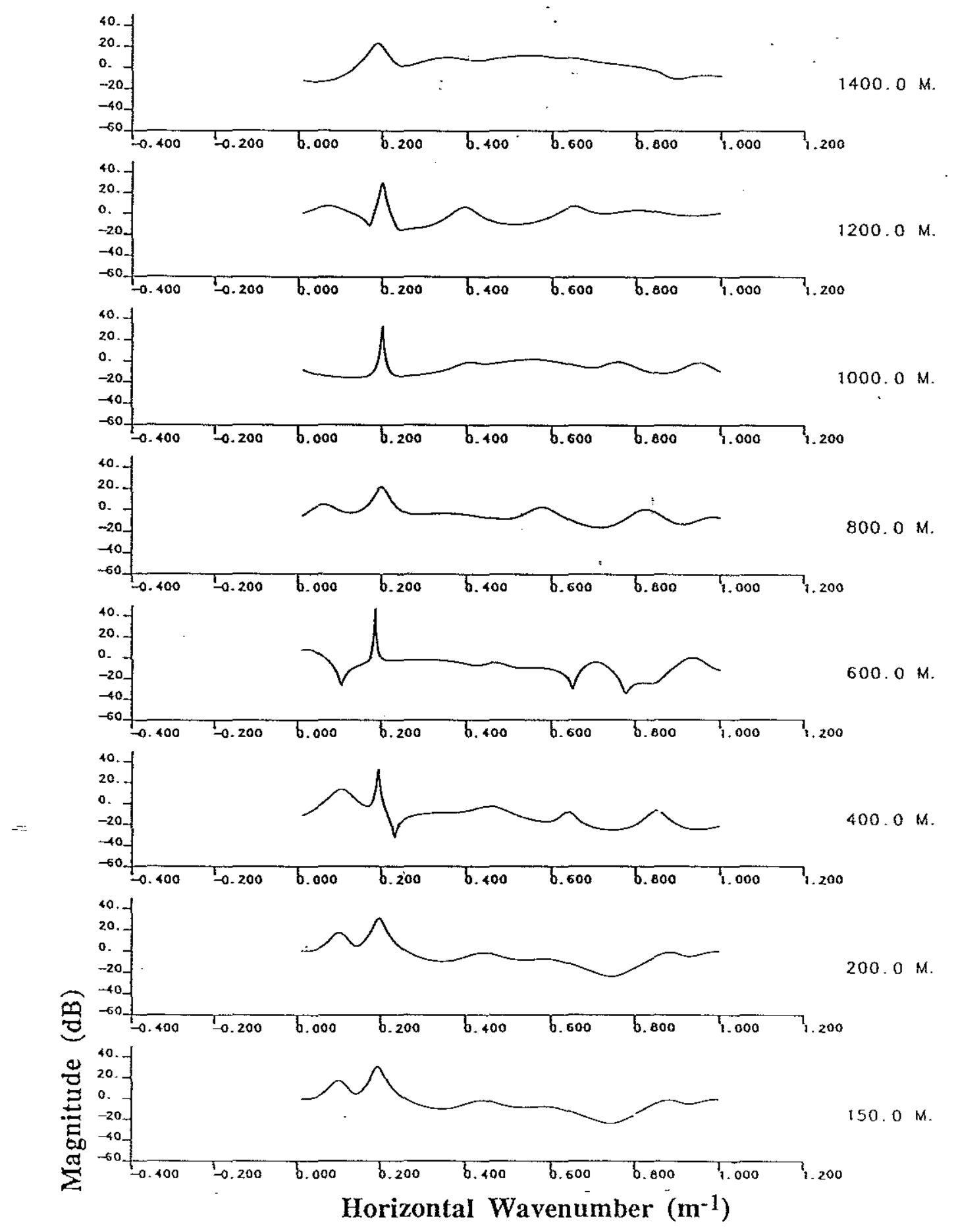

Fig 3.4.16 PRAWNS ESD for Corpus Christi $50 \mathrm{~Hz}$, lower hydrophone (100 pt, $50 \mathrm{pt}$ overlap, $1.23 \mathrm{~m}$ spacing, model order 25) 


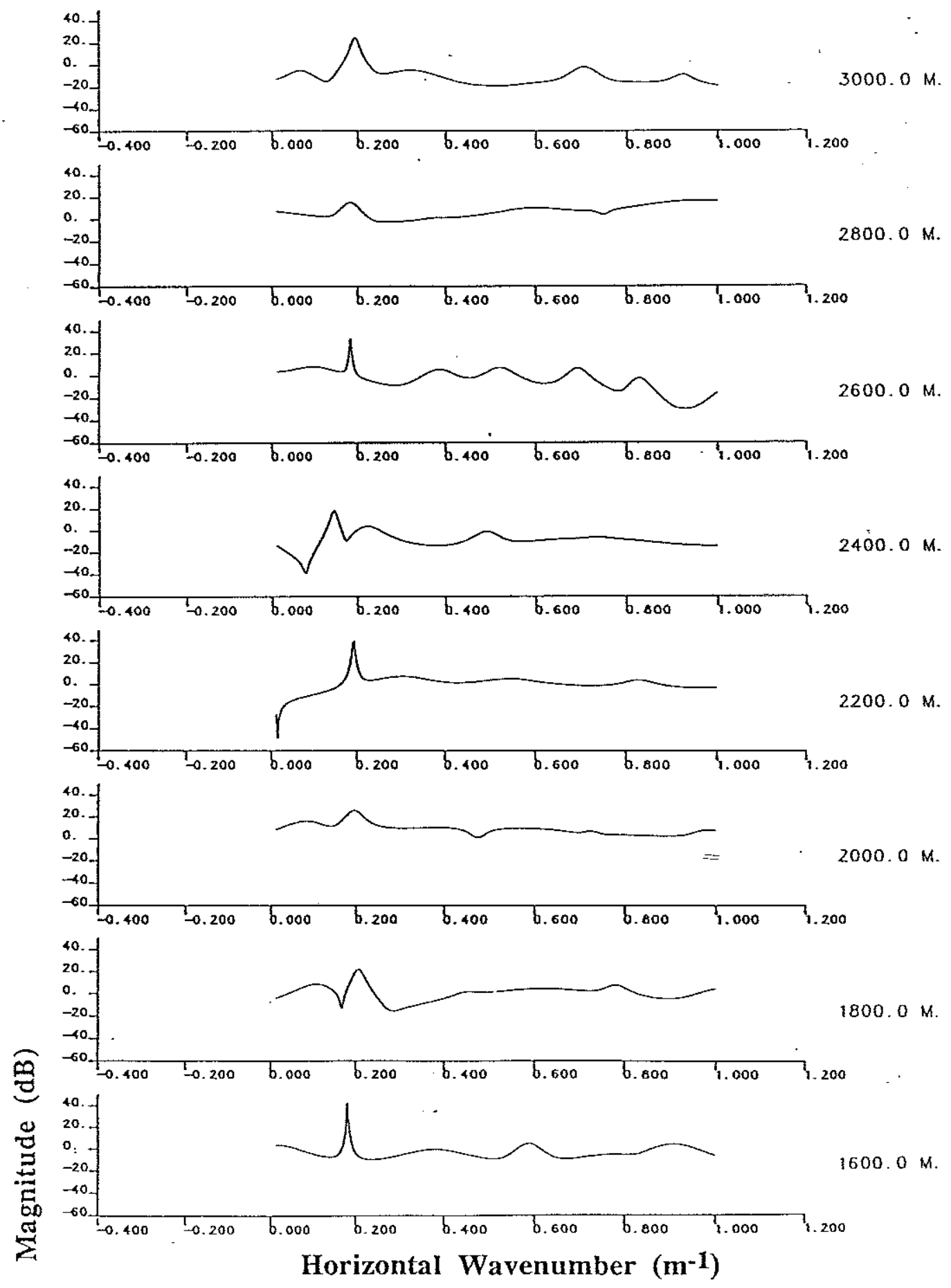

Fig 3.4.16(cont) PRAWNS ESD for Corpus Christi $50 \mathrm{~Hz}$, lower hydrophone (100 pt, $50 \mathrm{pt}$ overlap, $1.23 \mathrm{~m}$ spacing, model order 25) 


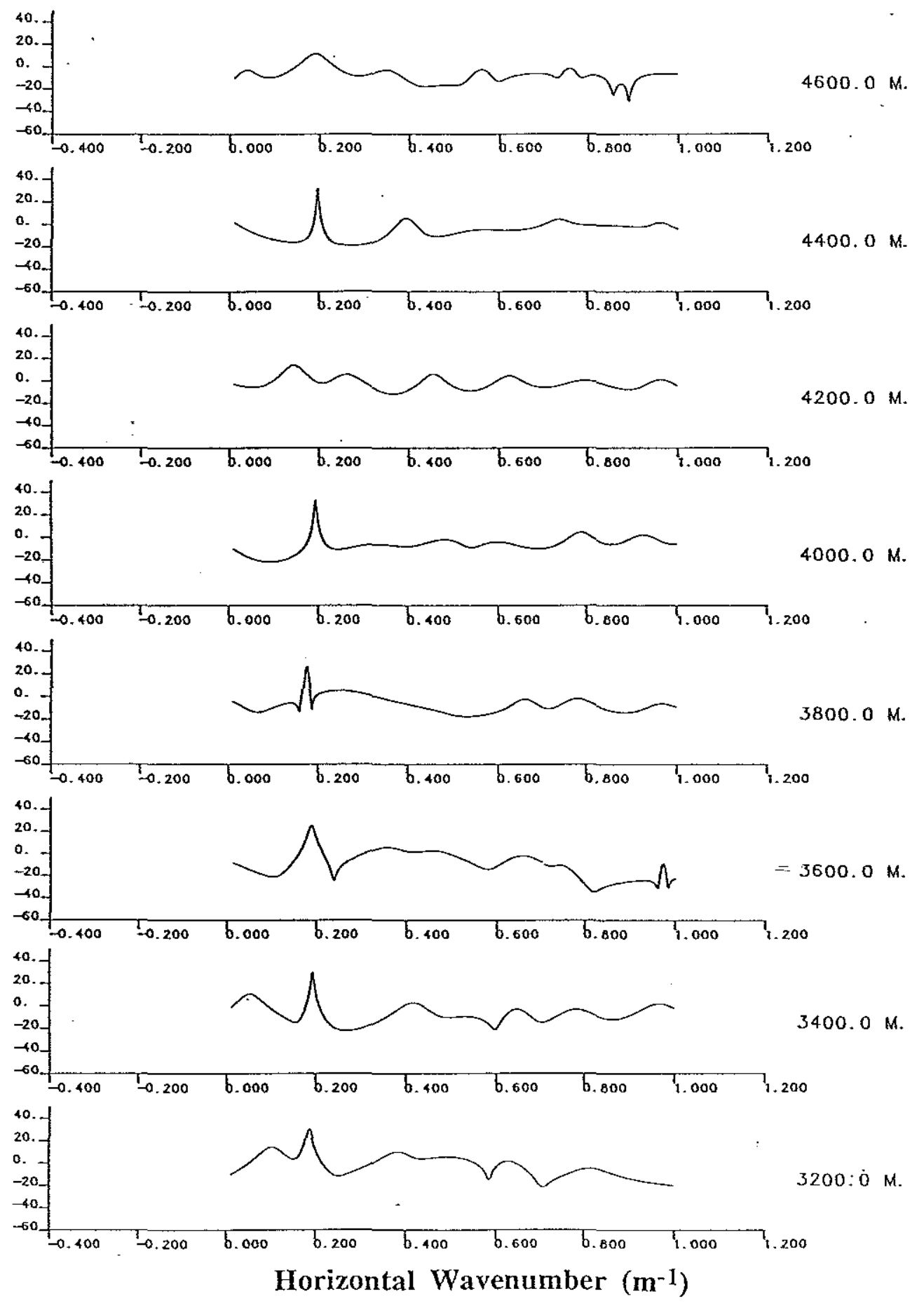

Fig 3.4.16(cont) PRAWNS ESD for Corpus Christi $50 \mathrm{~Hz}$, lower hydrophone (100 pt, 50 pt overlap, $1.23 \mathrm{~m}$ spacing, model order 25 ) 


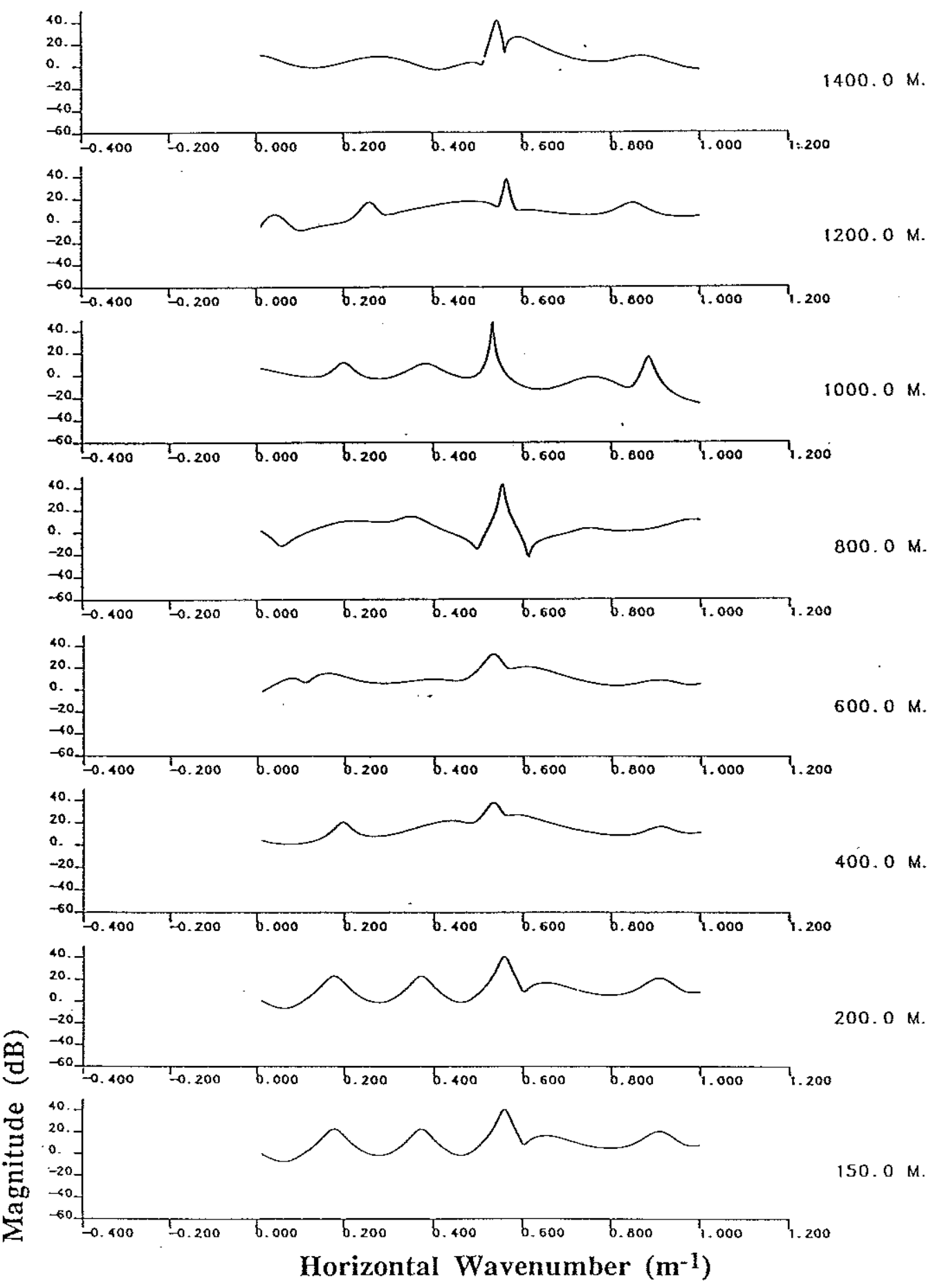

Fig 3.4.17 PRAWNS ESD for Corpus Christi $140 \mathrm{~Hz}$, upper hydrophone (100 pt, $50 \mathrm{pt}$ overlap, $1.23 \mathrm{~m}$ spacing, model order 25) 


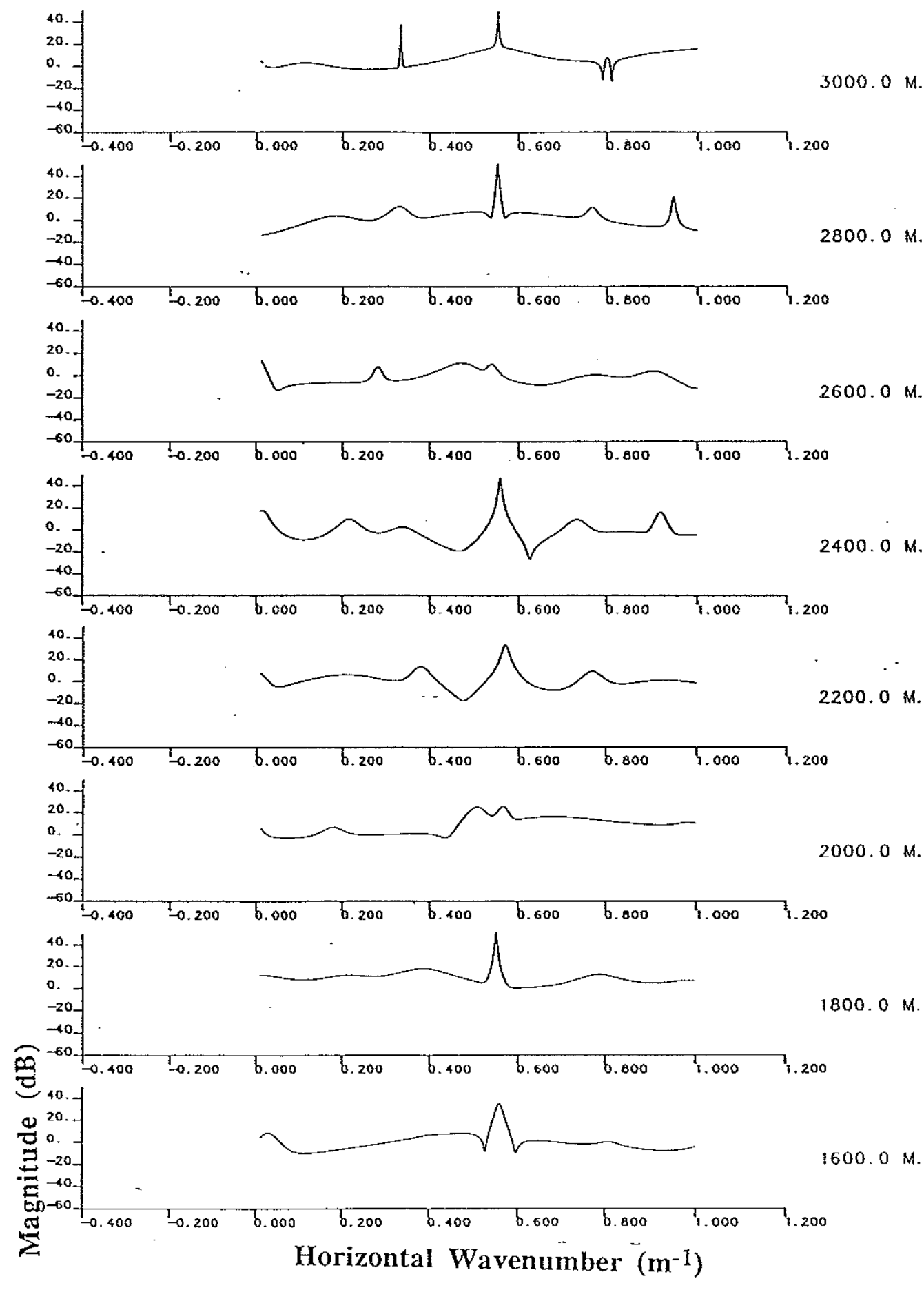

Fig 3.4.17(cont) PRAWNS ESD for Corpus Christi $140 \mathrm{~Hz}$, upper hydrophone (100 pt, 50 pt overlap, $1.23 \mathrm{~m}$ spacing, model order 25) 


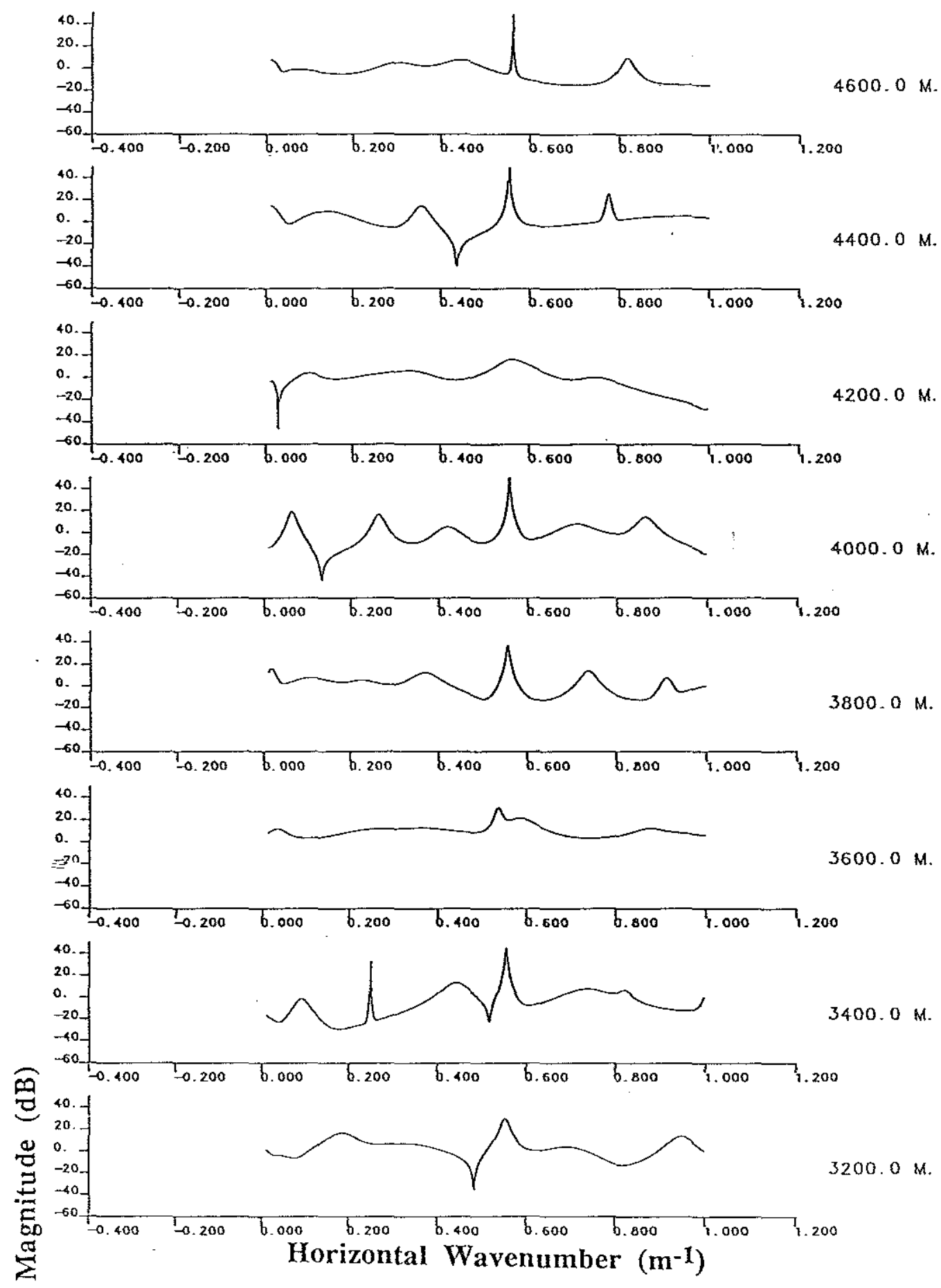

Fig 3.4.17(cont) PRAWNS ESD for Corpus Christi $140 \mathrm{~Hz}$, upper hydrophone (100 pt, $50 \mathrm{pt}$ overlap, $1.23 \mathrm{~m}$ spacing, model order 25) 

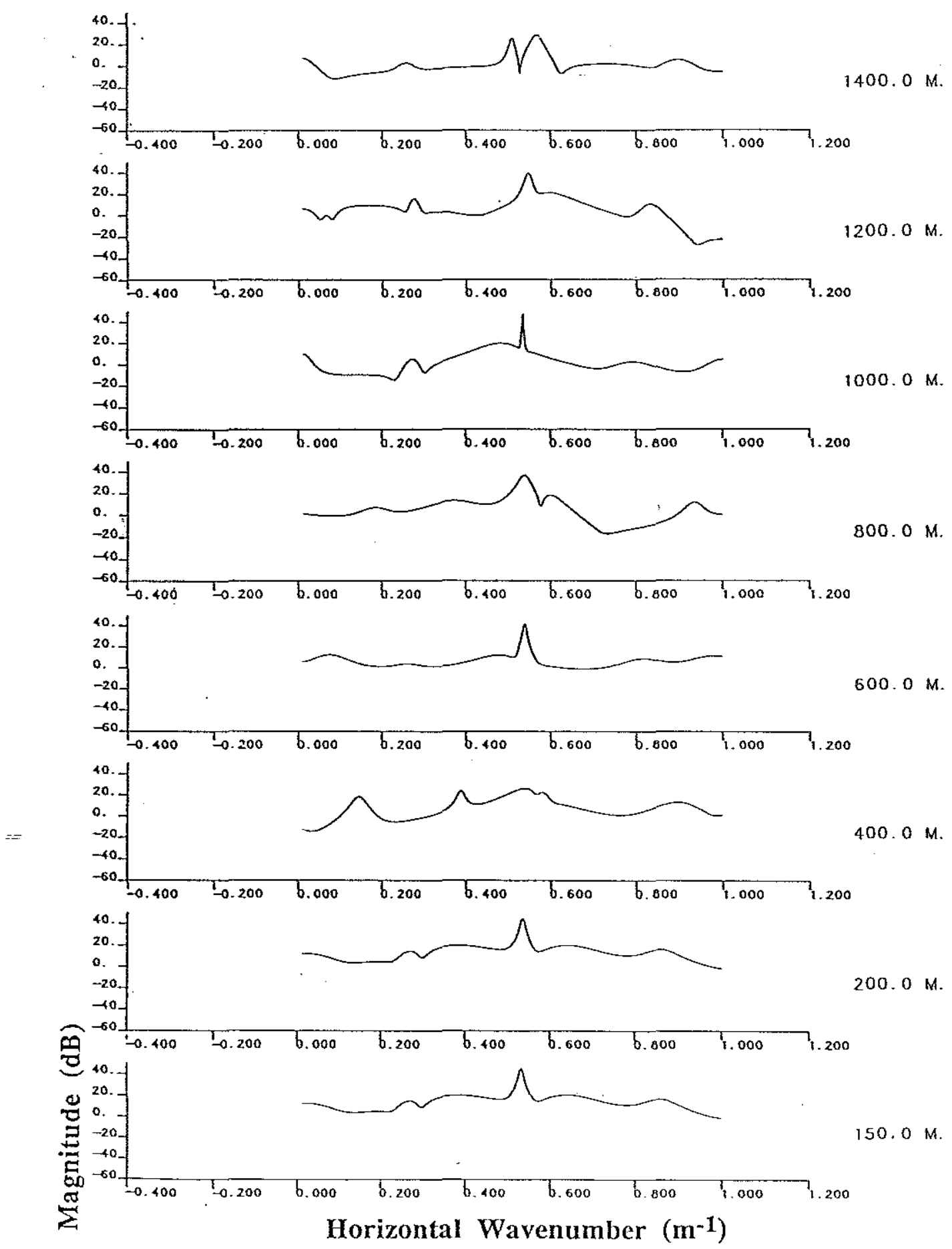

Fig 3.4.18 PRAWNS ESD for Corpus Christi $140 \mathrm{~Hz}$, lower hydrophone (100 pt, 50 pt overlap, $1.23 \mathrm{~m}$ spacing, model order 25) 


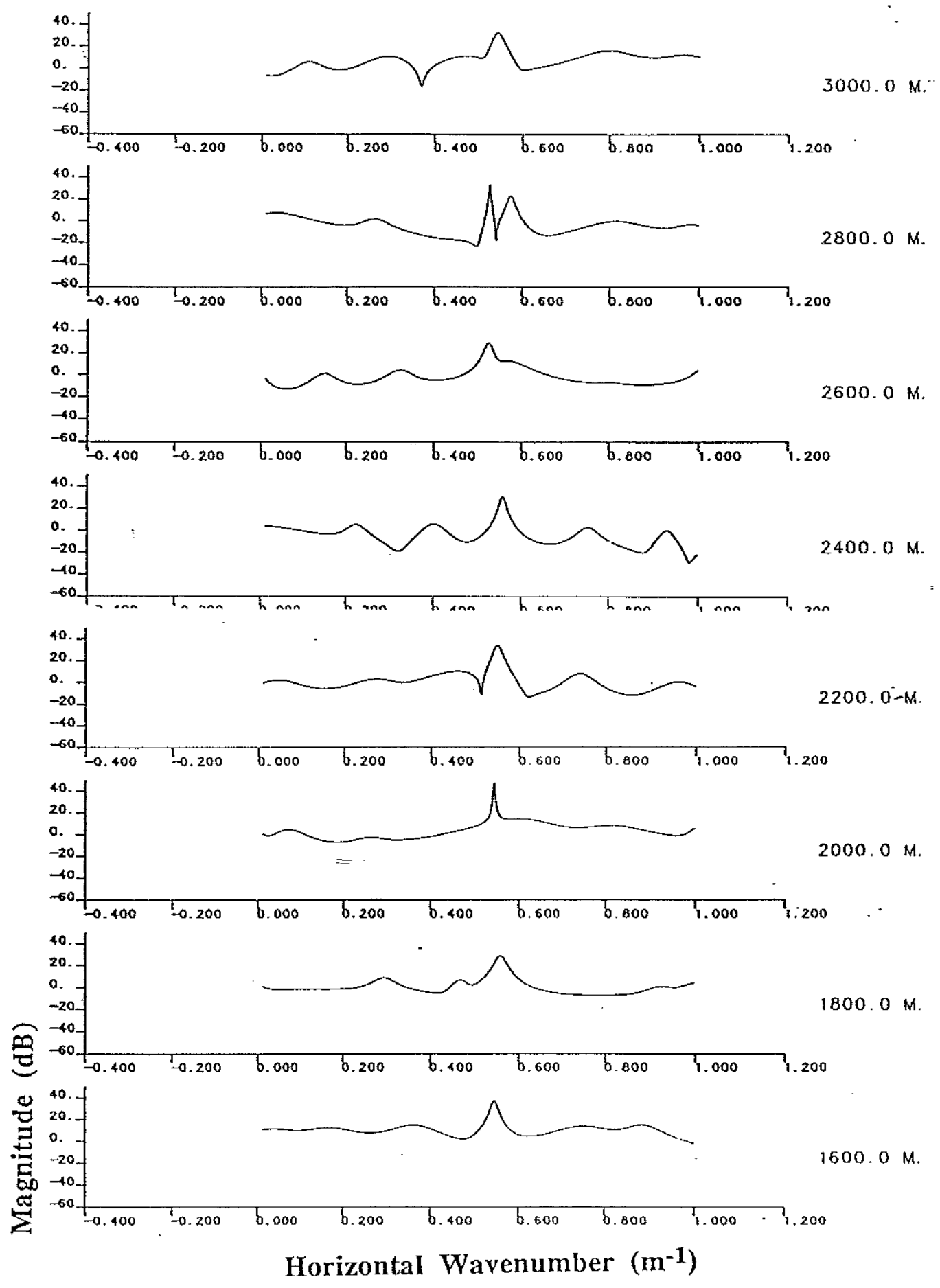

Fig 3.4.18(cont) PRAWNS ESD for Corpus Christi $140 \mathrm{~Hz}$, Iower hydrophone (100 pt, $50 \mathrm{pt}$ overlap, $1.23 \mathrm{~m}$ spacing, model order 25$)$ 


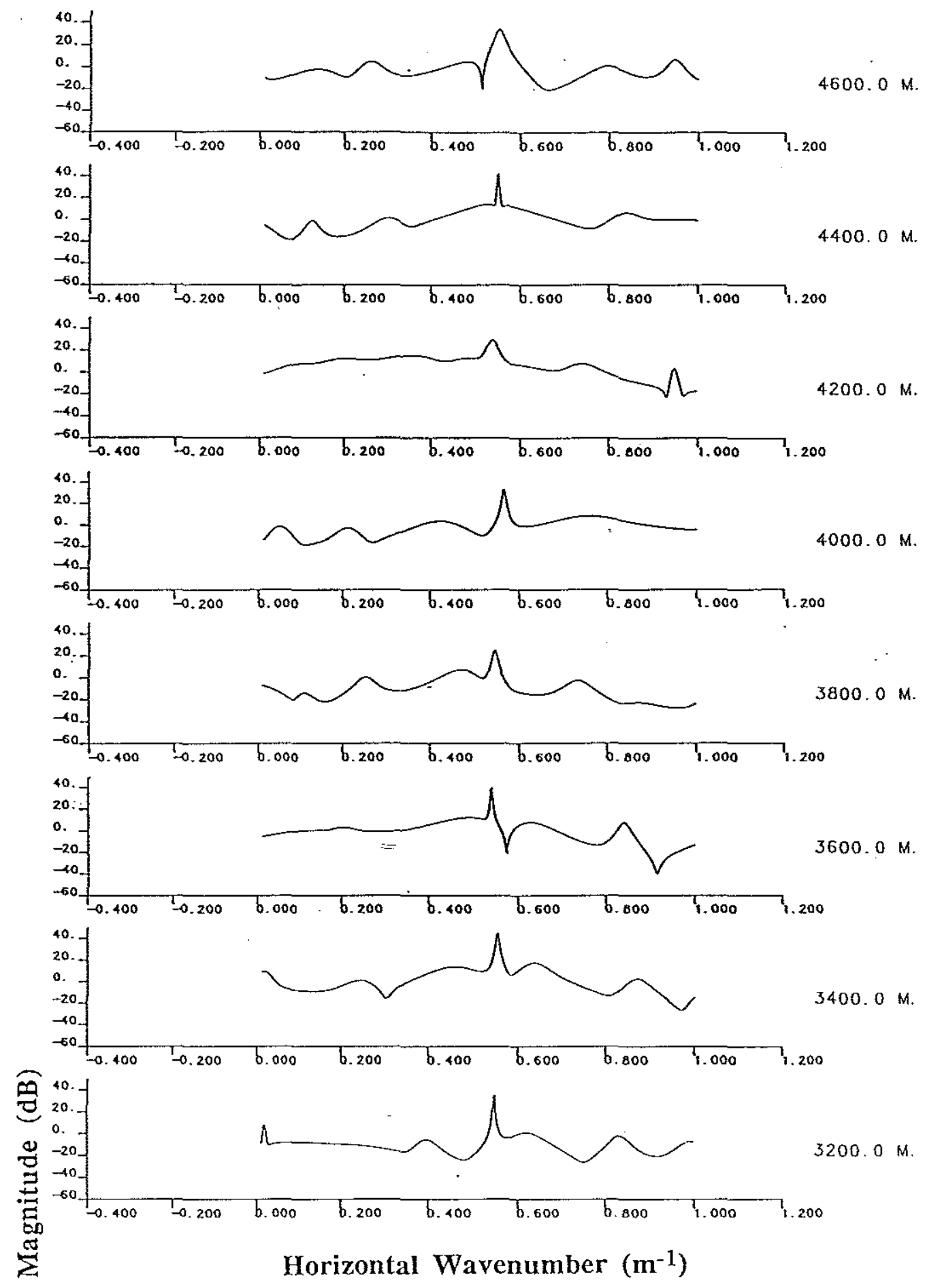

Fig 3.4.18(cont) PRAWNS ESD for Corpus Christi $140 \mathrm{~Hz}$, lower hydrophone $(100 \mathrm{pt}, 50 \mathrm{pt}$ overlap, $1.23 \mathrm{~m}$ spacing, model order 25) 


\subsection{Summary}

This chapter introduced the energy spectral density (ESD) which may be used as a tool similar to a plot of the Green's function. The Green's function may be modelled as an ARMA process while the ESD is an AR process. The advantage of the ESD in analysis using Prony's method is that the ESD provides a compact view of the effects of all of the estimated parameters in the range block of interest. The effect of high damping is a broad peak; since virtual modes may be modelled as highly damped propagating modes, limited tracking of virtual modes is possible.

Another tool used in the performance analysis is the residue which measures the fit of the observed data to an all pole model via an inverse filter. A low residue number indicates a good fit; this does not necessarily indicate accurate results. Empirically, it was found that examining the residue with respect to model order did result in good parameter estimates. While the plateau was inaccurate in the first case (Nantucket profile), it matched well with the the other model order selection effort in the second case (Corpus Christi). The second method for model order selection entailed observing pole "wander" and assumed the wavenumbers or poles would stop changing as model order was increased beyond a "breakpoint" order.

The sensitivity of the algorithm to input parameters leads to a group of empirically derived guidelines for applying Prony's method to a set of evenly spaced data. The first step is selection of a model order. Empirically, we found the model order should be two to three times the actual system order. This agrees with other investigators' results[32]. The actual system order may be obtained by inspection (from the numerically obtained Green's function) or numerically (SVD breakpoint identification). The first method is sensitive to aperture size and both of these approaches suffer in the presence of noise. The iterative schemes to obtain model order used in this study were based on residue and wavenumber evolution with changes in model order. Once the model order is identified, an aperture should be chosen. The processing block, as measured in points, must be more than the theoretical low limit of twice 
the model order, however, if the aperture is this small, the model order large and the data is noiseless, the matrix is likely to be singular. The upper limit of the aperture is the range interval of interest and the capabilities of the $\mathrm{QR}$ and rooting implimentations. The overlap and averaging are constrained by the processing and aperture sizes and are not particularly significant compared to the other parameters.

The Prony algorithm does suffer from noise corruption below $30 \mathrm{dBSNR}$. Since experimental data is frequently below this signal strength, some action is necessary to provide acceptable results. The runs made on the data demonstrate the correlation of results between the two hydrophones, but the ESD does not indicate the modes seen in the Green's function. One can either try more points (by splining), adjust the aperture and overlap/averaging or preprocess the data. The preprocessing may take the form of a filter or other noise reduction technique such as an SVD based scheme. Past research has demonstrated a tendency for the equations to become more ill conditioned as the sampling rate increases[63]. 


\subsection{Range Dependent Performance}

In the previous sections, we made the assumption that the environment was horizontally stratified. This range independent constraint is not particularly limiting although natural occurances of pure stratification are unlikely. This section will examine the performance of the Prony algorithm in two cases of waveguide range dependence[68]. The justification for application of the high resolution technique is based on finding local modes in an adiabatic environment.

A phenomenon associated with range dependent waveguides is coupled modes, in which the energy of a particular mode may be transferred to another mode[59,61]. A brief development of the theory will serve to identify the consequences of ignoring mode coupling. Using the ability to expand an arbitrary function in terms of eigenfunctions, we define

$$
\mathrm{p}(\mathrm{r}, \mathrm{z})=\sum_{\mathrm{n}=1}^{\infty} \mathrm{R}_{\mathrm{n}}(\mathrm{r}) \phi_{\mathrm{n}}(\mathrm{z}, \mathrm{r})
$$

If the assumed solution is substituted into the equation:

$$
\nabla^{2} \mathrm{p}(\mathrm{r}, \mathrm{z})+\mathrm{k}^{2}(\mathrm{r}, \mathrm{z}) \mathrm{p}(\mathrm{r}, \mathrm{z})=0
$$

and angular symmetry is assumed, then the equation in cylindrical coordinates is:

$$
\sum_{n}\left[\frac{\partial^{2} R_{n}}{\partial r^{2}} \phi_{n}+2 \frac{\partial R_{n} \partial \phi_{n}}{\partial r \partial r}+\frac{R_{n} \partial \phi_{n}}{r \partial r}+\frac{\phi_{n} \partial R_{n}}{r \partial r}+R_{n} \frac{\partial^{2} \phi_{n}}{\partial r^{2}}+R_{n}\left(\frac{\partial^{2} \phi_{n}}{\partial z^{2}}+k_{m}^{2} \phi_{n}\right)\right]=0
$$

Utilizing the properties of a complete orthonormal set, the orthogonal eigenfunctions will satisfy:

$$
\int_{0}^{\infty} \rho(\mathrm{z}) \phi_{\mathrm{n}}(\mathrm{z}, \mathrm{r}) \phi_{\mathrm{m}}(\mathrm{z}, \mathrm{r}) \mathrm{dz}=\delta_{\mathrm{nm}}
$$

Multiplying equation (4.1.3) by $\rho(\mathrm{z}) \phi_{\mathrm{m}}(\mathrm{z}, \mathrm{r})$ and integrating with respect to depth, we obtain: 


$$
\frac{\mathrm{d}^{2} \mathrm{R}_{\mathrm{m}}}{\mathrm{dr}^{2}}+\frac{1}{\mathrm{r}} \frac{\mathrm{dR} \mathrm{R}_{\mathrm{m}}}{\mathrm{dr}}+\mathrm{k}_{\mathrm{rn}}^{2} \mathrm{R}_{\mathrm{m}}=-\sum_{\mathrm{n}}\left\{\mathrm{A}_{\mathrm{mn}}(\mathrm{r}) \mathrm{R}_{\mathrm{n}}+\mathrm{B}_{\mathrm{mn}}(\mathrm{r})\left[\frac{\mathrm{R}_{\mathrm{n}}}{\mathrm{r}}+2 \frac{\mathrm{dR} \mathrm{R}_{\mathrm{n}}}{\mathrm{dr}}\right]\right\}
$$

where $A_{m n}(r)$ and $B_{m n}(r)$ are coupling coefficients defined as:

$$
\begin{aligned}
& A_{m n}(r)=\int_{0}^{h} \frac{1}{\rho_{0}(z)} \frac{\partial^{2} \phi_{n}(z, r)}{\partial r^{2}} \phi_{m}(z, r) d z \\
& B_{m n}(r)=\int_{0}^{h} \frac{1}{\rho_{0}(z)} \frac{\partial \phi_{n}(z, r)}{\partial r} \phi_{m}(z, r) d z
\end{aligned}
$$

This yields the set of coupled equations for $R_{m}(r)$. The boundary conditions become more complex and are stated by Boyles as the radiation condition in the form[14]:

$$
\lim _{r \rightarrow \infty} \sqrt{r}\left[\frac{d R_{n}}{d r}-j k_{r n} R_{n}\right]=0
$$

and for the source located at $\mathrm{r}=0$ :

$$
\lim _{\varepsilon \rightarrow \infty}\left[\varepsilon \frac{d R_{n}(\varepsilon)}{d r}\right]=\frac{\phi_{n}(0,0)}{2 \pi \rho_{0}(0)}
$$

The coupled equations describe the exchange of energy between the modes. As the medium approaches the horizontally stratified model of the previous sections, the coupling coefficients approach zero[59].

In certain situations, mode coupling is assumed not to occur; this is known as the adiabatic approximation. Each mode retains its initial energy; if the mode is cutoff, it's energy is lost rather than transferred to the propagating modes. By an adiabatic change in a parameter, we mean the parameter does not vary locally; for example, using sound speed, $c$, as the adiabatic parameter:

$$
\begin{aligned}
& \frac{\partial c}{\partial r} \rightarrow 0 \quad \text { locally and } \\
& \int_{0}^{R} \frac{\partial c}{\partial r} \mathrm{dr}=\Delta \mathrm{c} \text { for a large } \mathrm{R}
\end{aligned}
$$


This assumption holds quite well in slowly changing environments. An additional assumption made in the derivation of coupled mode theory is that of angular independence (cylindrical symmetry). This is not strictly true, such as in a coastal wedge situation, but the approximation is frequently used because of the small slopes involved.

A solution to equation (4.1.2) may be found by applying the WKB approximation in range to the differential equation for $R$. The WKB method assumes a slowly varying medium and neglects the second derivative terms since they are negligible with respect to the lower order terms. Defining a new function, $F_{n}(r)=\sqrt{r} R_{n}(r)$ and applying the adiabatic approximation (no mode coupling) the new equation is:

$$
\frac{\partial F_{m}(r)}{\partial r}+\left[k_{m}^{2}(r)+\frac{1}{4 r^{2}}\right] F_{m}(r)=0
$$

By neglecting the second term in the brackets and using the asymptotic form of the Hankel function, the acoustic pressure field is[62]:

$$
\left.p(z, r)=\sqrt{\frac{2}{\pi} e^{-j \frac{\pi}{4}}} \sum_{n} u_{n}\left(z_{0}, 0\right) u_{n}(z, r) \frac{1}{\sqrt{k_{\mathrm{rn}} \mathrm{r}}} e^{(j} \int^{r} k r n d r\right)
$$

The exponential term with the integral is a phase accumulation mechanism. As the $\mathrm{k}_{\mathrm{rn}}$ change for each range interval of interest, the rate of phase accumulation changes.

The application of Prony's method to small range apertures is made within the context of the adiabatic approximation. The parameters estimated and the ensuing ESD are valid only for the interval of interest; there is no system constraint to join the analysis of one section to that of adjoining range intervals. Within each range interval, the waveguide parameters, including bathymetry, are assumed to be constant. Changes in the waveguide boundary conditions will result in a smearing of the estimated parameters and ESD.

Conventional spectrum estimation techniques are frequent victims of changes in waveguide boundary conditions over the interval of interest. In Fourier techniques, the resolution is related to the length of the data segment. In order to achieve high resolution, large 
apertures are used; the assumption of constant boundary conditions and environmental conditions under these circumstances is poor. The application of high resolution techniques to the same environment permits a smaller aperture to be used and the locally range independent assumptions should be easier to justify.

In an effort to examine the performance of the Prony algorithm in an environment with range dependencies, two test environments are developed. The first contains a bathymetry change; it consists of a parallel plate region which evolves into an upslope wedge section. In the second, the bathymetry is constant while the bottom parameters undergo a step change at a given range. The fields for both of these examples were generated using a parabolic equation approach in which the elliptic Helmholtz equation is approximated by a parabolic equation[64-66]. 


\subsection{Ramp Example}

The first test of the adiabatic modes was conducted using a parallel plate region abutting a wedge. The environment is the same as that used by Jensen and Kuperman in their analysis of sound propagation in a wedge shaped ocean with a penetrable bottom[66].

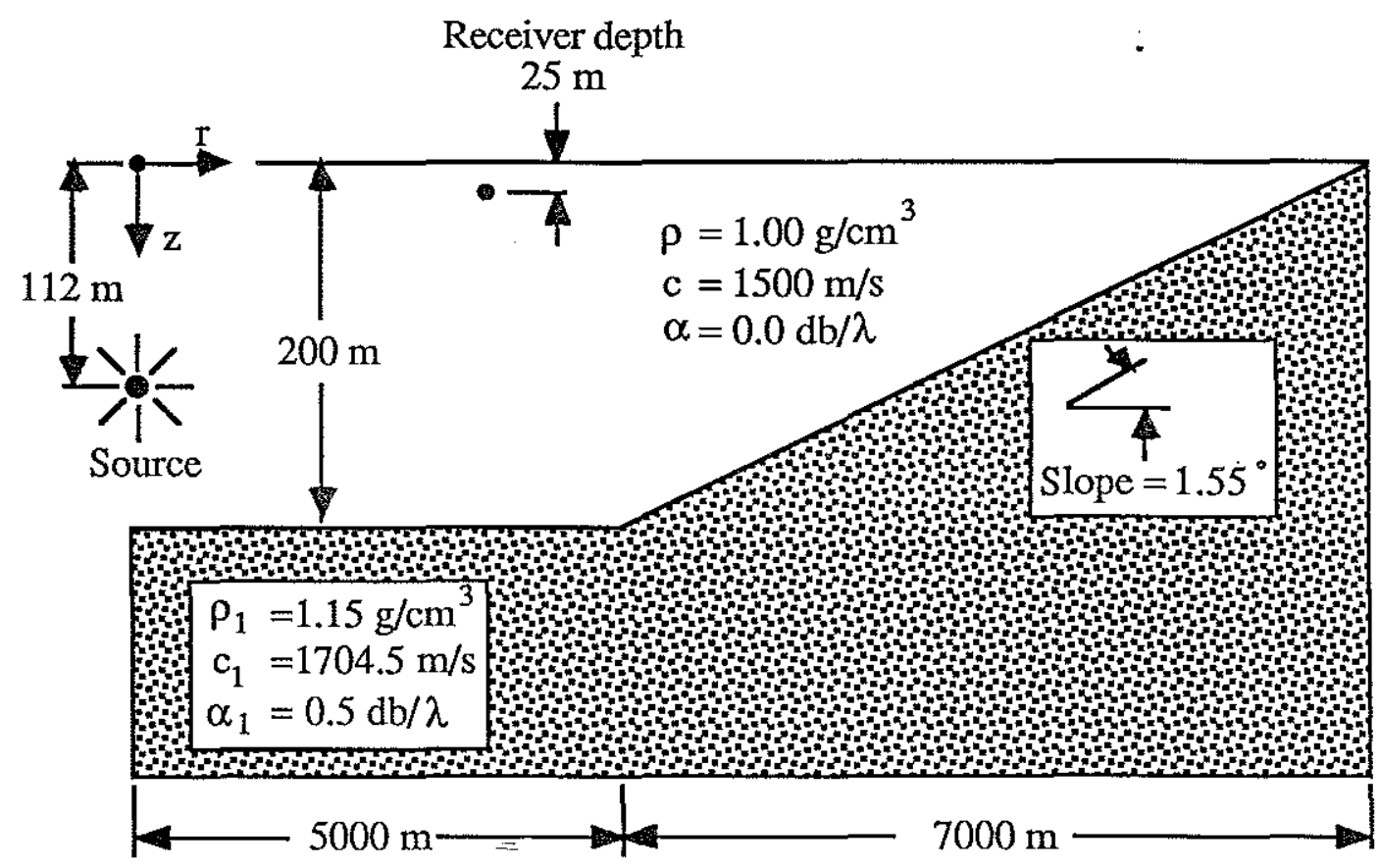

Fig 4.2.1 Coastal Wedge Geometry

The placement of the source and receiver is such that there are two modes excited; the first and third mode propagate while the source is in the null of the second mode. As stated in the previous chapter, adiabatic mode propagation is assumed; ie, mode coupling is not considered. This assumption is plausible with the small slope and widely separated wavenumbers.

Providing a valid reference spectrum in the range dependent environment is a difficult task for two reasons. First, since the range dependent field generation is done through an approximation method (the parabolic equation), the field generation program in addition to the analysis algorithm may introduce output aberrations. Second, the range dependent nature of the waveguide raises the question as to the definition of an appropriate reference spectrum [63]. 
As outlined in section 4.1 , the adiabatic approximation is used. Since the bottom velocity profile used in this case is an isovelocity one, the Pekeris waveguide was used as a reference for wavenumber accuracy. For each processing block, the mean depth of the range interval was used as the depth of a Pekeris waveguide with bottom parameters identical to the wedge. Given the source-receiver geometry, waveguide depth and the bottom parameters, the local Pekeris waveguide may be analyzed for the "reference" wavenumbers. The Prony wavenumbers plotted are the parameter outputs from the algorithm. The graph of figure 4.2.2 used a 250 meter aperture and a model order of five. In the initial 1500 meters, the startup phenomenon of the PE approximation method causes the aberrations and oscillatory behavior. Once the field generation program stabilizes, the agreement between the Prony's method and the Pekeris reference is quite good. An interesting trait of the algorithm is the limited ability to track modes past cutoff. These virtual modes may be modelled as highly damped modes $[19,20,67]$.

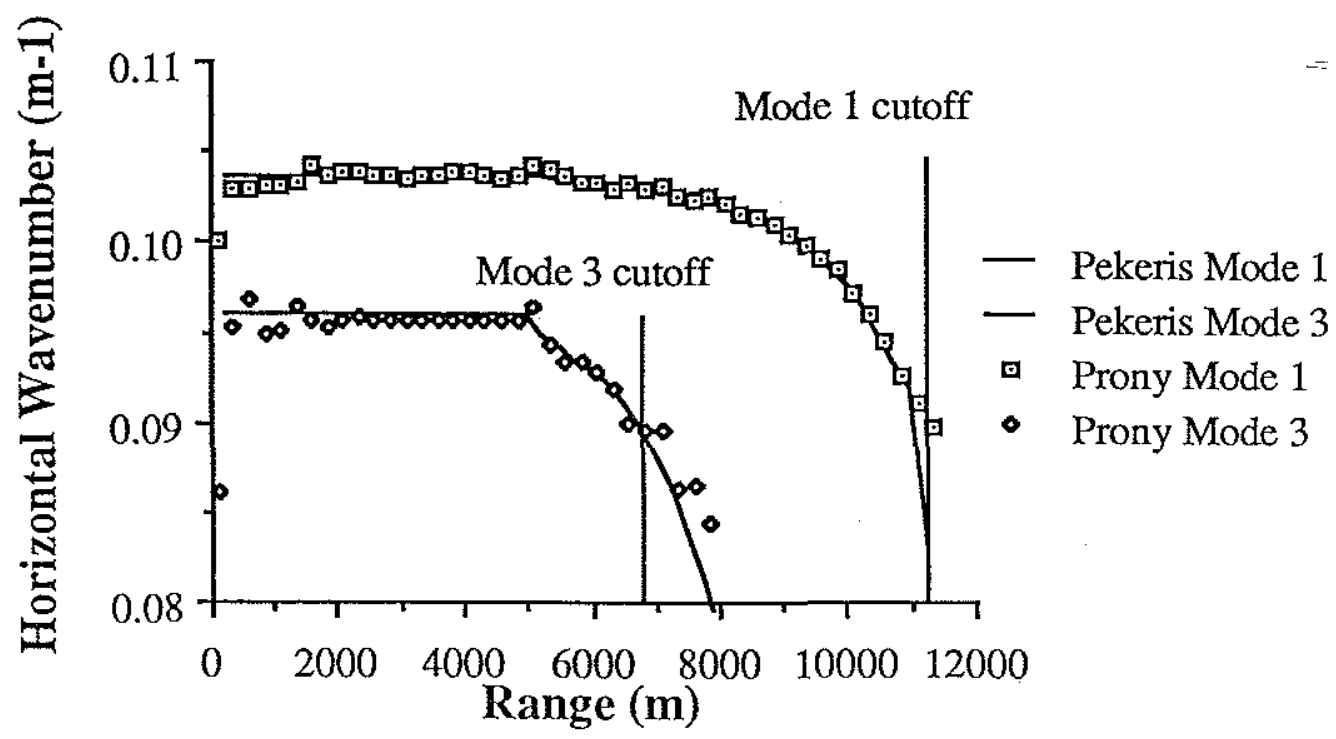

Fig 4.2.2 Pekeris vs PRAWNS analysis for coastal wedge 
The energy from the virtual modes is "dumped" into the penetrable bottom; the detectable amplitude will significantly decrease for each range block until that mode can no longer be detected.

As the specified model order varies, the residue should decrease somewhat as the larger model order is available to account for the moving average portion of the system. Graphs of the residues(figures 4.2.3 and 4.2.4) for several orders show this general trend. By holding the other parameters constant ( $250 \mathrm{~m}$ aperture or averaging block with a processing block of 50 points at 5 meter spacing, no overlap) and varying the model order, the effect of the specified number of modes becomes clear. As the amount of overspecification increases, the system more closely matches the all pole environment. In the residue graphs for model orders 4 and 6 , the best match to an all pole system is in the region from 3000 to 5000 meters after the PE generating algorithm has stabilized and before the upslope region. A model order of 10 or 20 yields a better match across the entire range block. The cause of large residue spikes in the $4000 \mathrm{~m}$ range interval of the large order models is not known. 

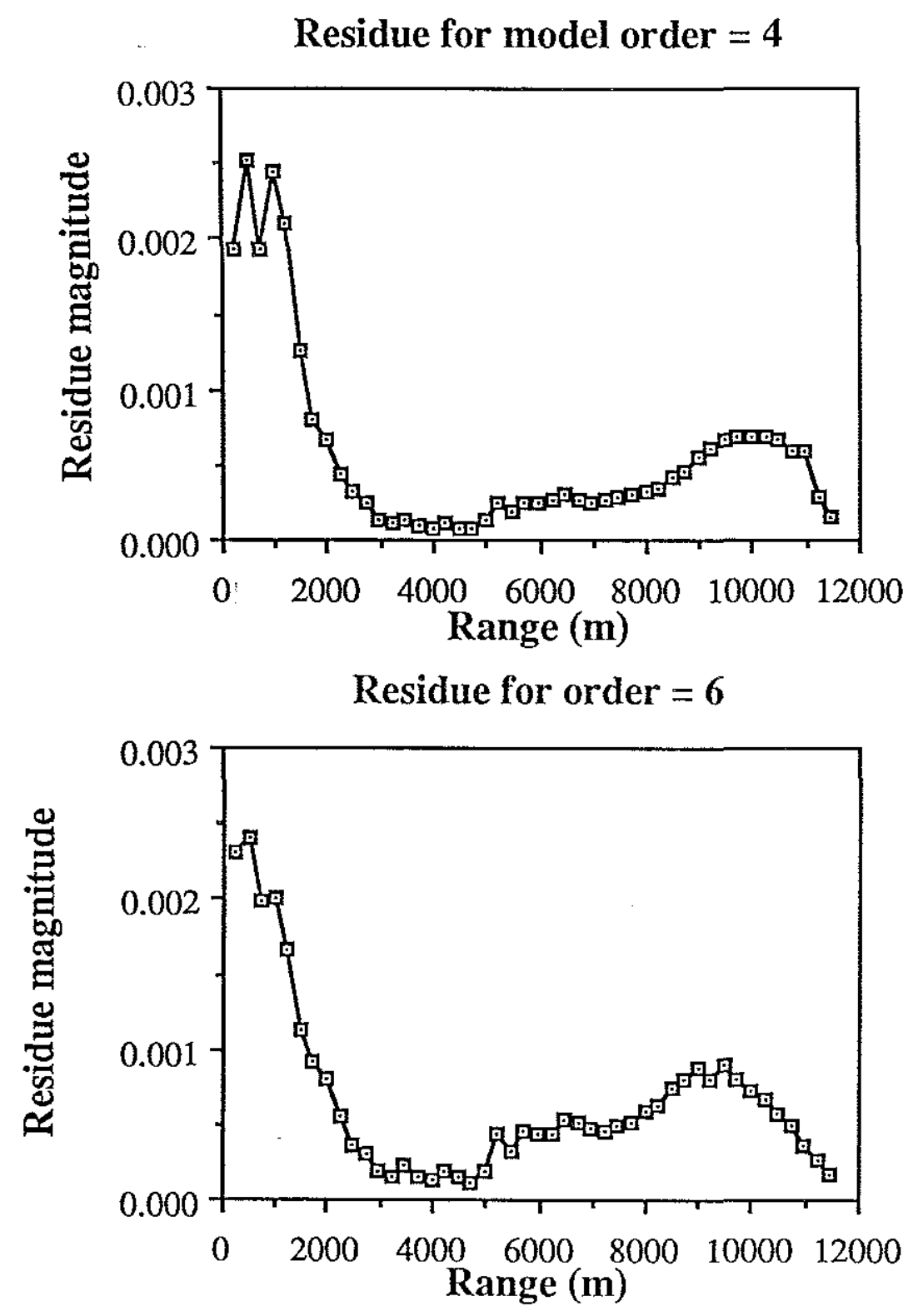

Fig 4.2.3 Residue graphs for ramp model orders 4 and 6 

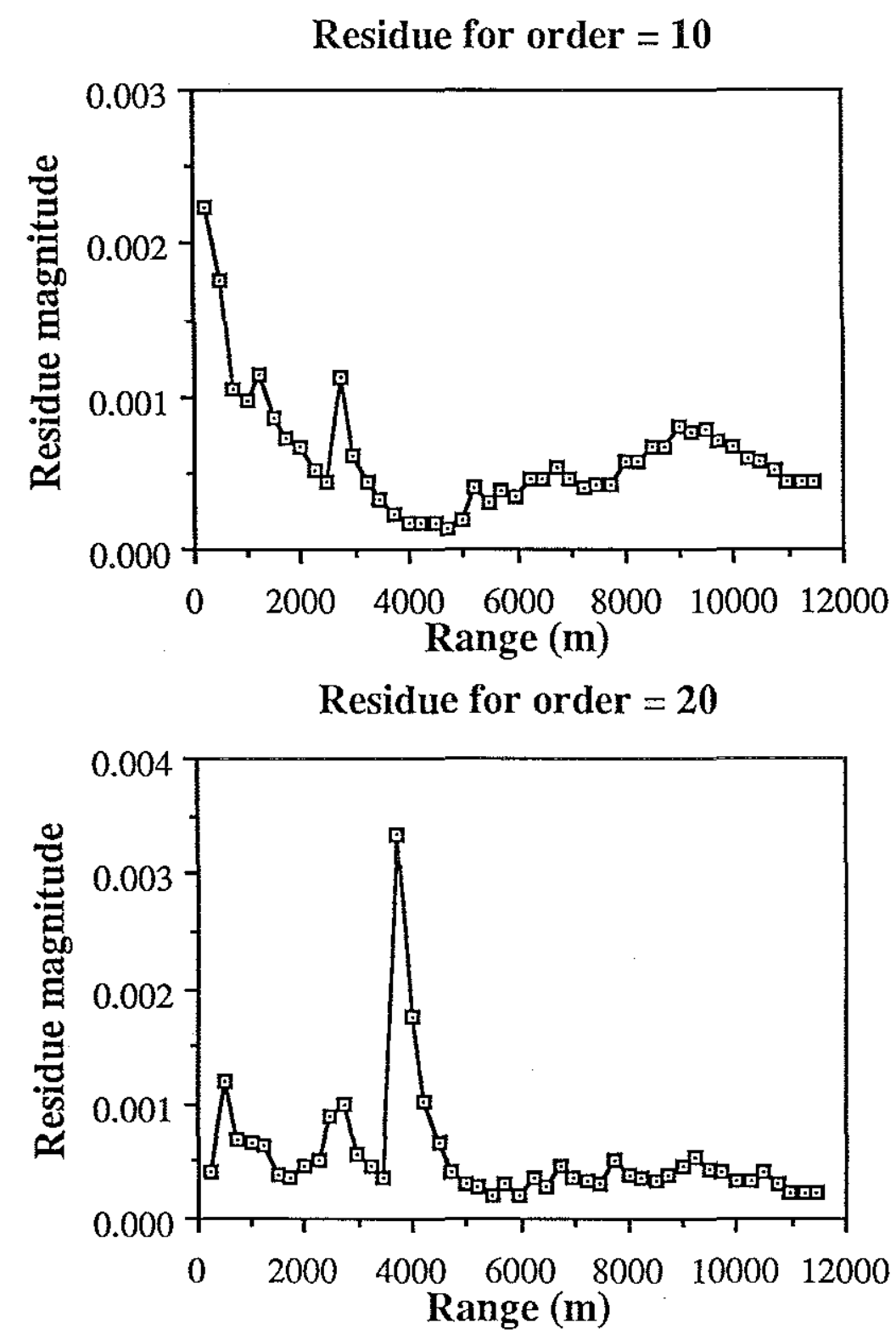

Fig 4.2.4 Residue graphs for ramp model orders 10 and 20

The use of this type of residue is not, however, the only consideration for selecting the "best" model order for the problem. In figures 4.2.5 and 4.2.6, the small residues associated with the exact model order and the associated wavenumber graph are shown. While the residues have small magnitudes over the entire range interval, the accuracy of the estimated wavenumbers does not match the over specified model order evaluations of the same environment. Even a graph of the total residue (fig 4.2.7), obtained by summing the individual 
range block residues does not show the drawback of using a model order equal to the exact system order. The total residue does indicate the general "better fit" of the higher order models.

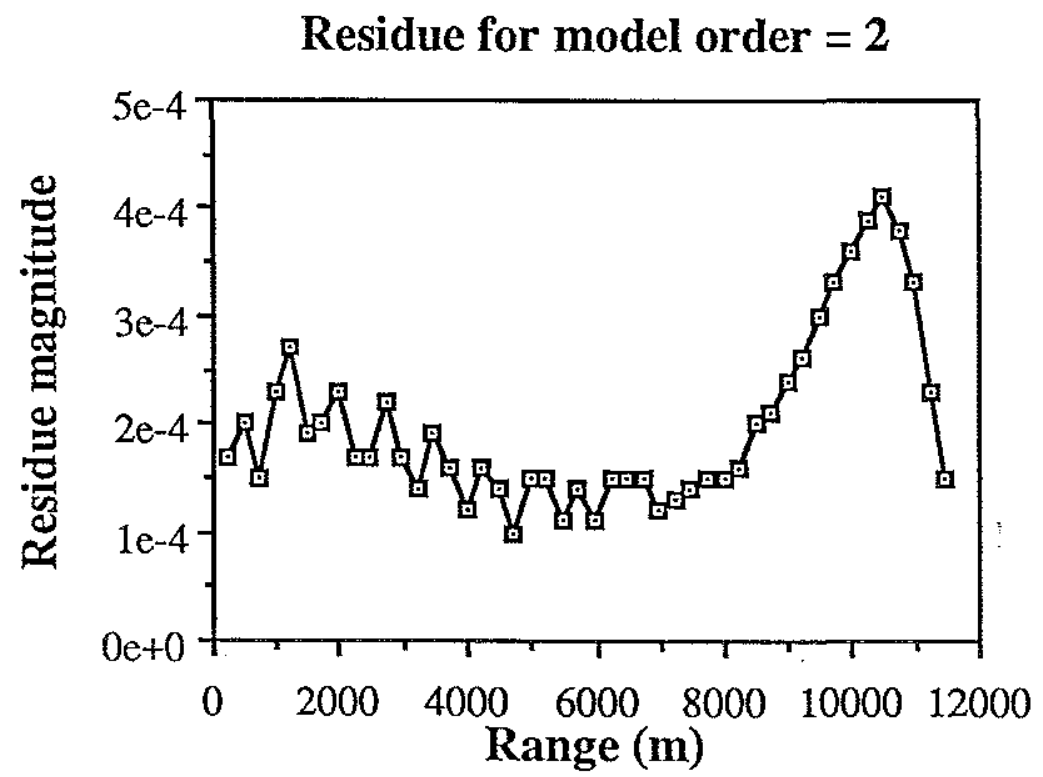

Fig 4.2.5 Residue vs range for ramp model order $=2$

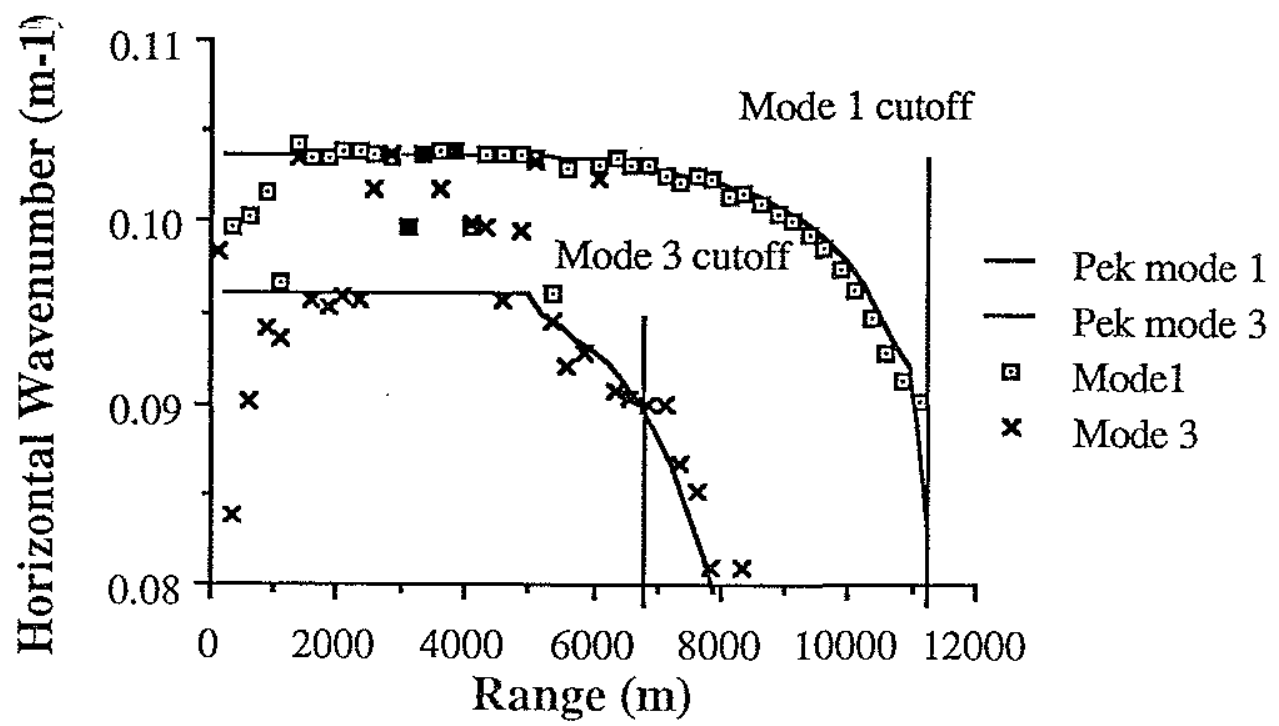

Fig 4.2.6 PRAWNS vs. Pekeris for ramp model order $=2$ 


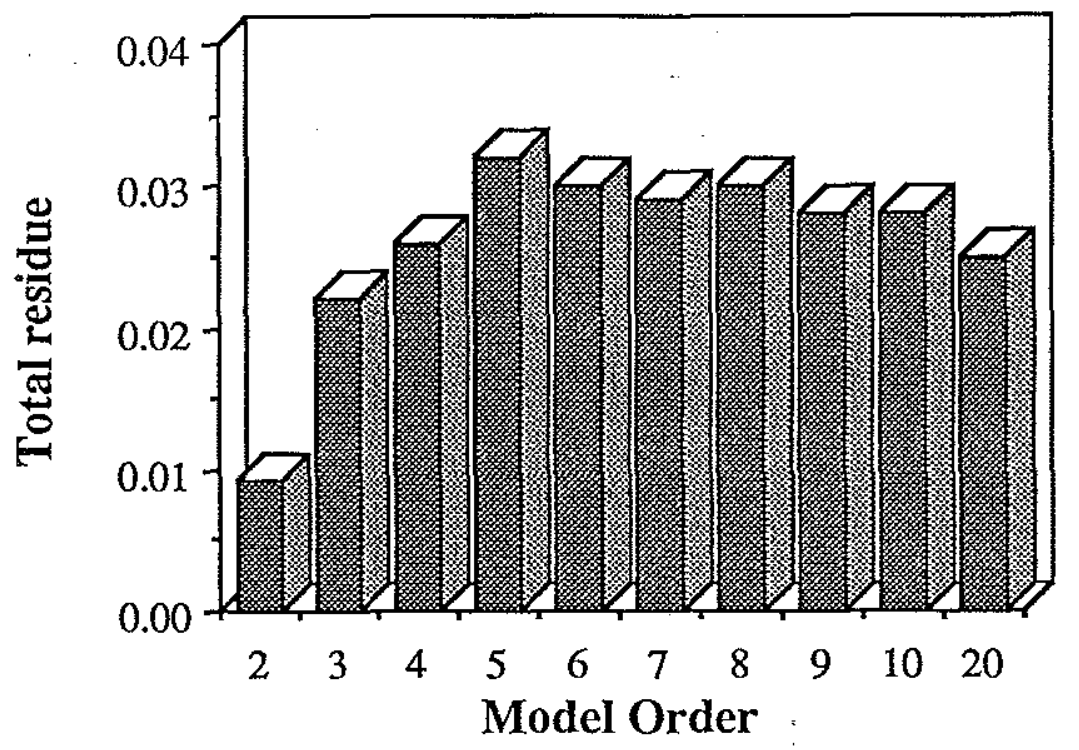

Fig 4.2.7 Total residue sum vs. model order

The ESD is particulary useful in examining the changes in the modal structure in the range dependent waveguide. Figure 4.2.8 demonstrates this utility; it is a progressive collection of ESD for various range blocks. The transformation of the tabular data into a spectal presentation allows a physical interpretation of the propagating field. The initial startup

$=\quad$ of the PE code (PAREQ) is evident as is the "steady state" condition in the parallel plate region. The upslope transition at 5000 m causes the mode energy to move toward cutoff. After mode 3 cuts off, this peak becomes broad as energy is being dissipated(into the bottom) from the mode. The location of the peak energy in the remaining mode moves toward lower wavenumbers (and cutoff). The mode shape is compressed as the channel dimensions narrow causing the peak energy in mode 1 to increase. The ESD plot in figure 4.2 .8 indicates that both modes have cutoff by $11500 \mathrm{~m}$. 


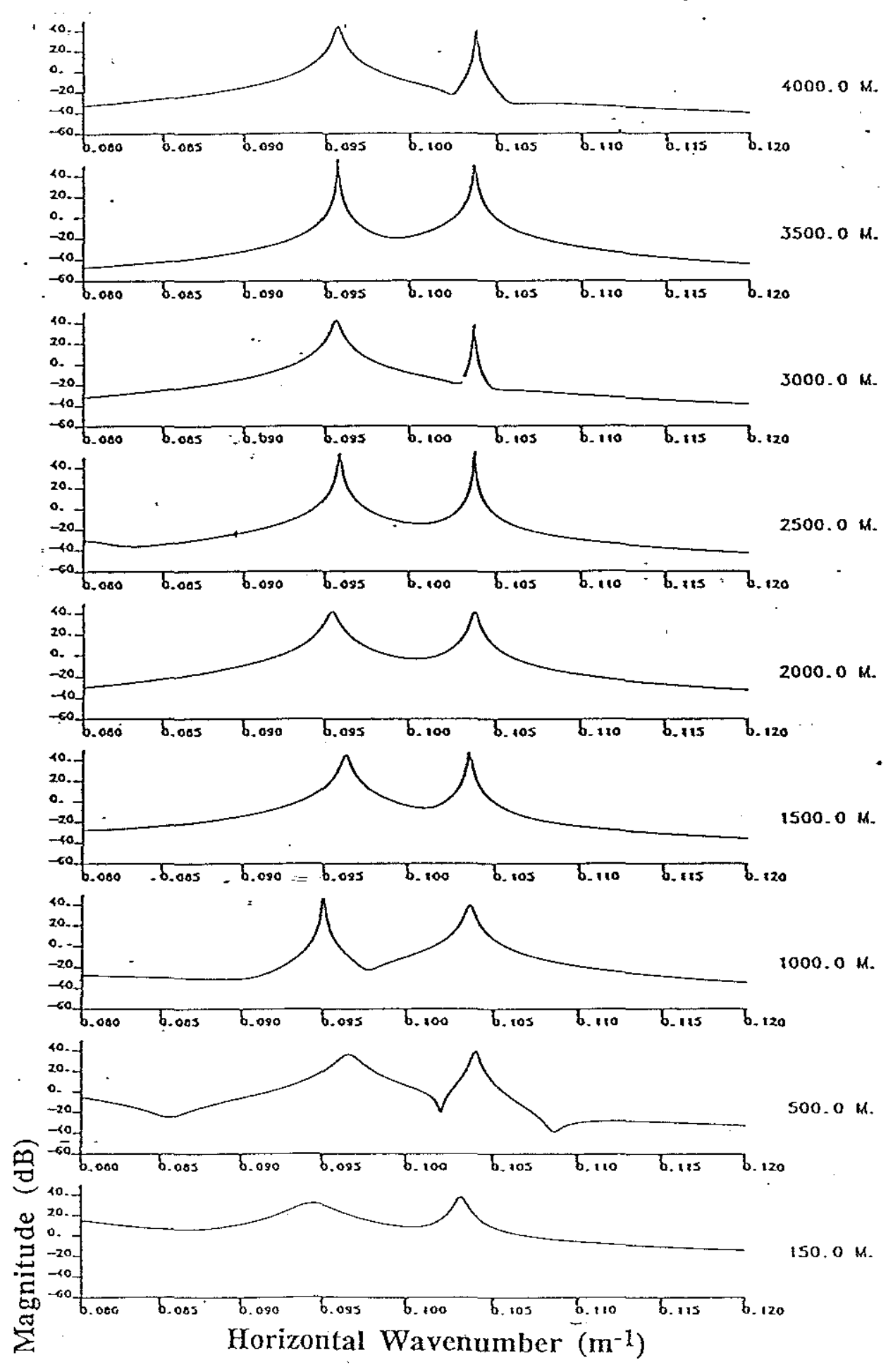

Fig 4.2.8 PRAWNS ESD for ramp example $(50$ pt, $5 \mathrm{~m}$ spacing, 0 overlap, model order 20$)$ 


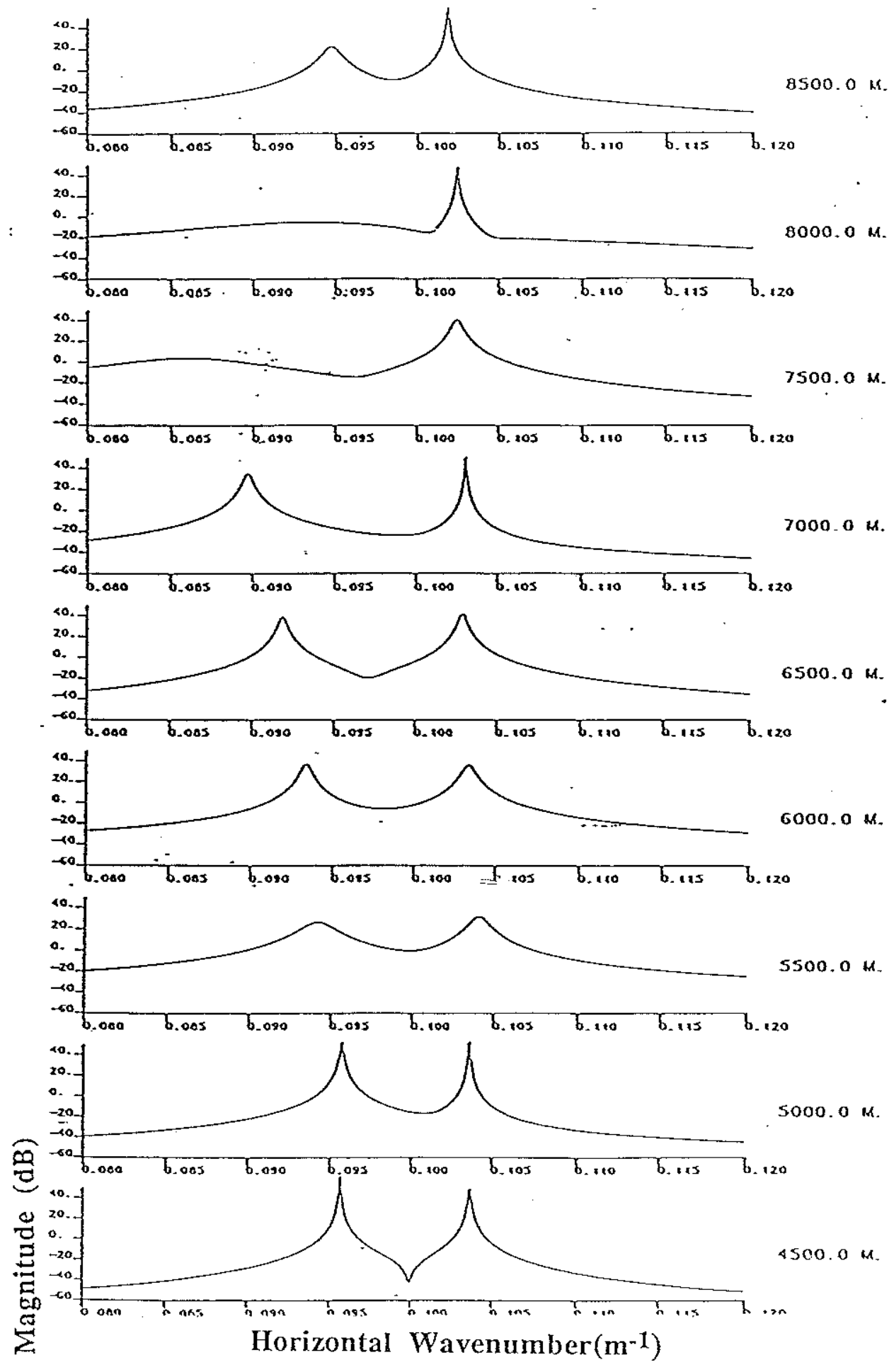

Fig 4.2.8(continued) PRAWNS ESD for ramp example (50 pt, $5 \mathrm{~m}$ spacing, 0 overlap, model order 20 ) 


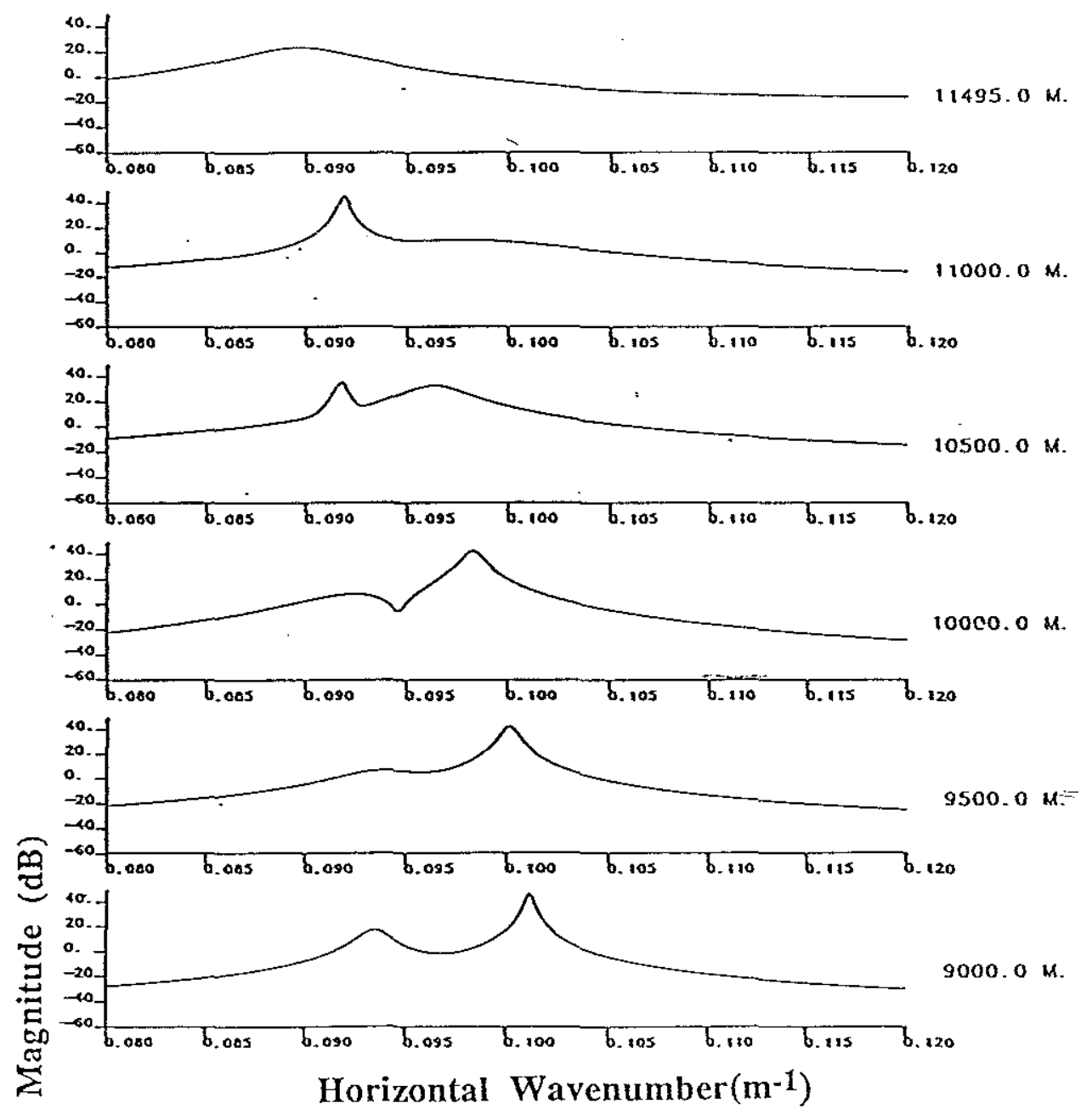

Fig 4.2.8(continued) PRAWNS ESD for ramp example $(50 \mathrm{pt}, 5 \mathrm{~m}$ spacing, 0 overlap, model order 20 ) 
By collecting the ESD for each range block and integrating the results in a contour plot, the shift in wavenumber and changes in energy level are summarized in figure 4.2.9.

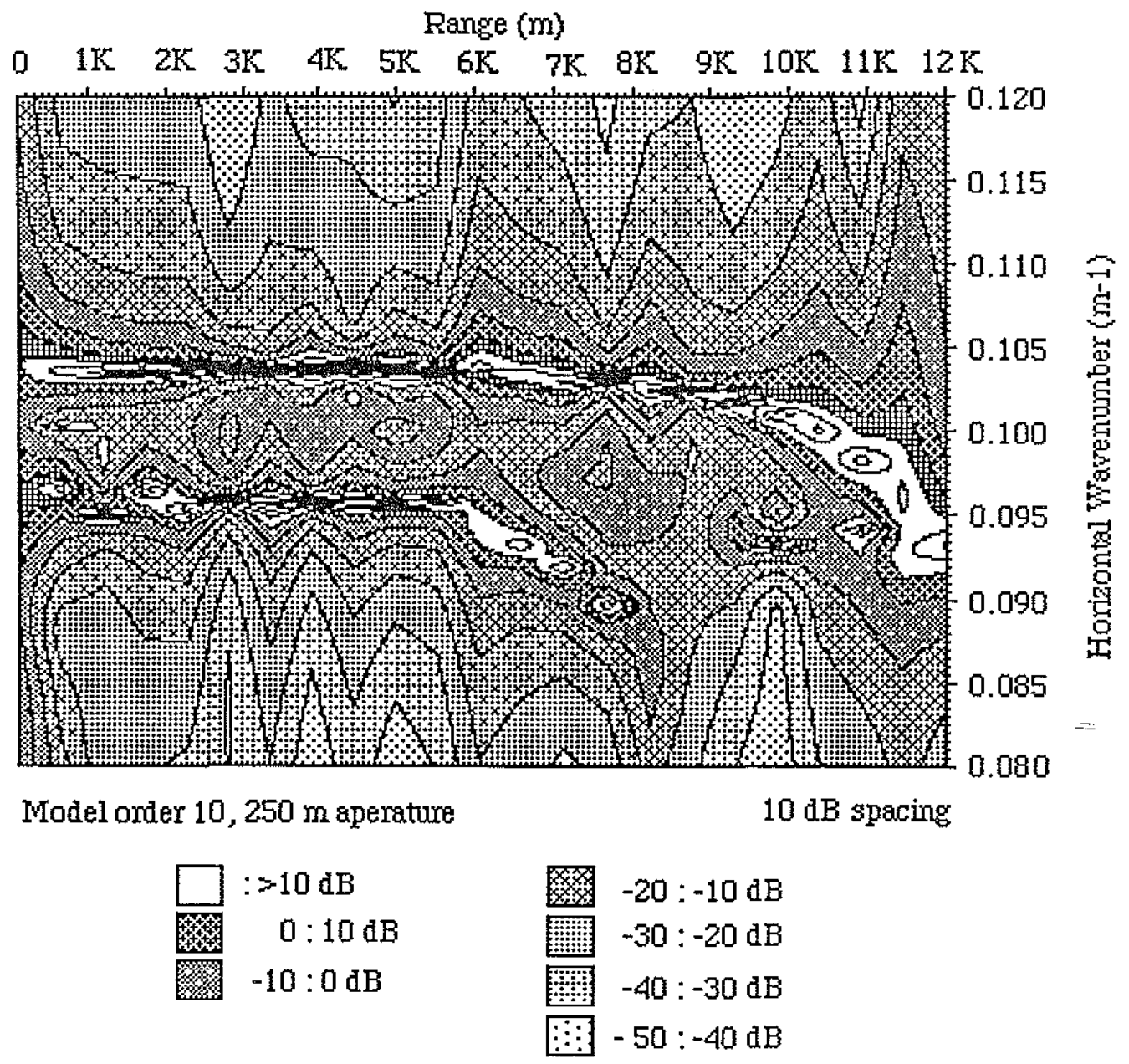

Fig 4.2.9 Contour plot of ramp example 


\subsection{Geoacoustic Parameter Shift Example}

The second environment for an evaluation of the Prony's method in a range dependent waveguide is a parallel plate region with a step change in bottom sound speed and density. The values used in the bottom intervals are from Hamilton's compilation of bottom types and their representative parameters[69]. Again, the source-receiver geometry is such that there are two modes propagating in the water column; the source is in the null of mode 2 , which does not propagate. The bottom variation was chosen to force one of the modes into cutoff.

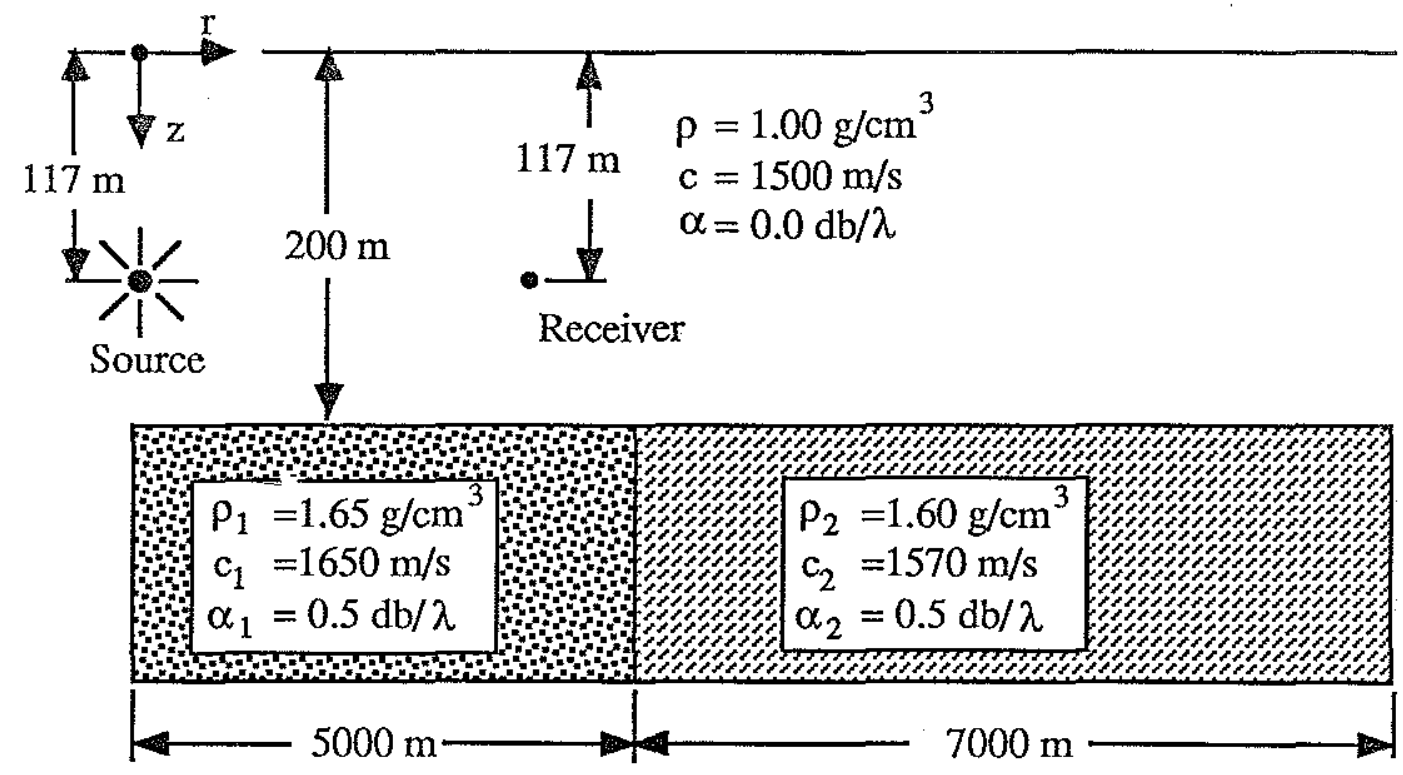

Fig 4.3.1 Bottom Parameter Shift Example

The performance of the Prony algorithm with model order changes for this waveguide was similar to that of the ramp example of the last section. The graph of figure 4.3.2 shows the close tracking of the PRAWNS output with the Pekeris reference. The wavenumber amplitude 
tended to die out reasonably quickly. Figure 4.3.2 also indicates the startup phenomenon of the PE equation method used to generate the field.

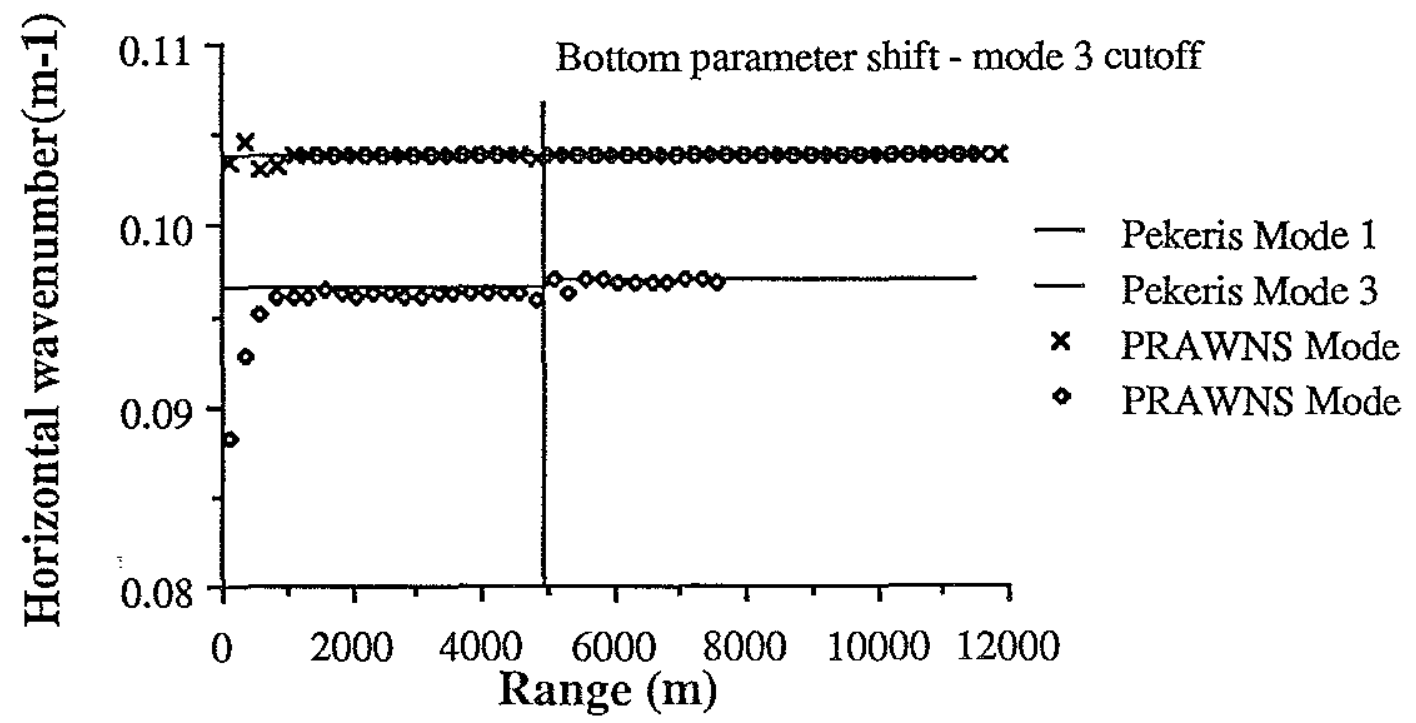

Fig 4.3.2 Pekeris vs PRAWNS analysis for parameter step shift

In figure 4.3 .2 , the shift in mode 1 is obscured by the symbols used to mark the PRAWNS output. The shift in the mode 1 wavenumber is small; the initial Pekeris wavenumber is $.103874 \mathrm{~m}^{-1}$ while the Pekeris wavenumber after the bottom shift is $.103958 \mathrm{~m}^{-1}$. The algorithm tracks this change but even the difference plot of figure 4.3.3 does not clearly indicate the error ( the error is the difference between the Pekeris and PRAWNS values in a given range interval). The average error magnitude for mode 1 is $4.3 \mathrm{E}-05$ and for mode 3 it is 3.6 E-04. 


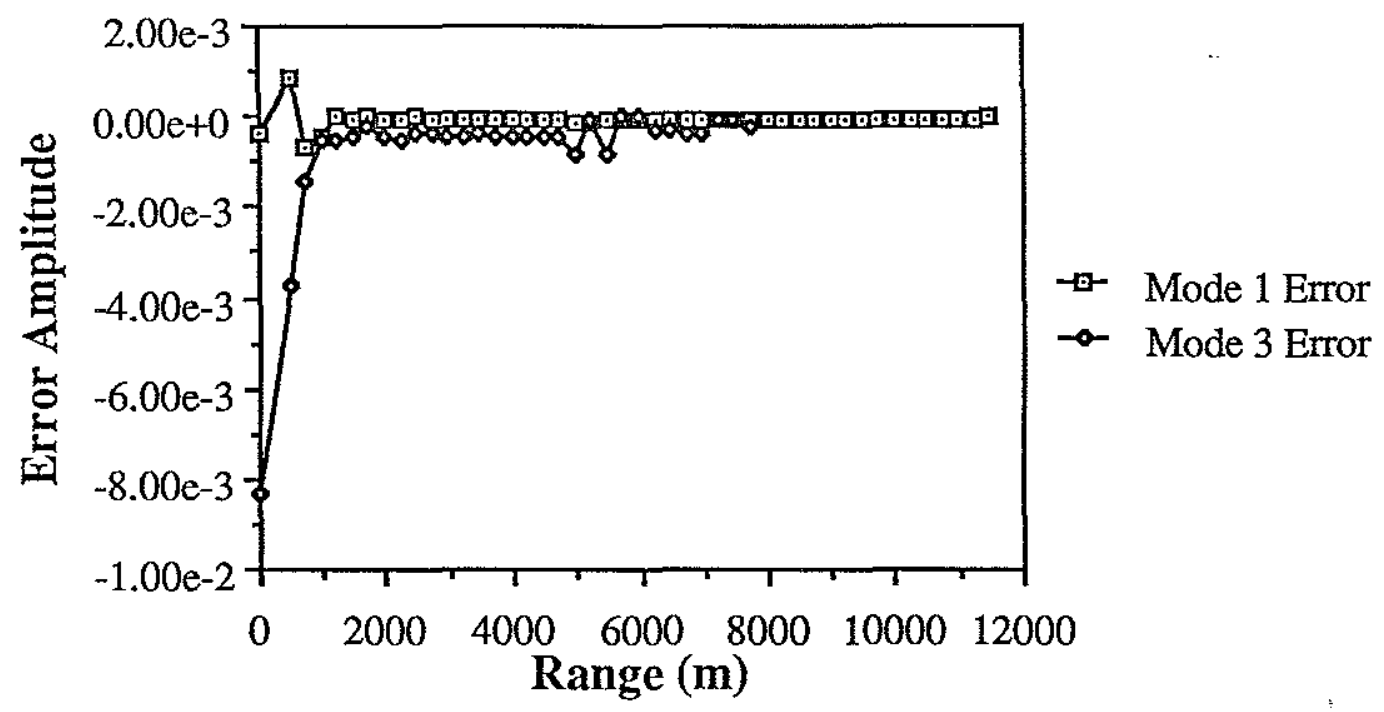

Fig 4.3.3 Wavenumber error vs. range for bottom shift model (50 pt, $5 \mathrm{~m}$ spacing, 0 overlap, model order 20 )

The use of the residue as an analytical tool in this environment is summarized in figures 4.3.4 and 4.3.5. The residue decreases for higher order model but again the larger order models have residue spikes in the region prior to the wavenumber shift. 

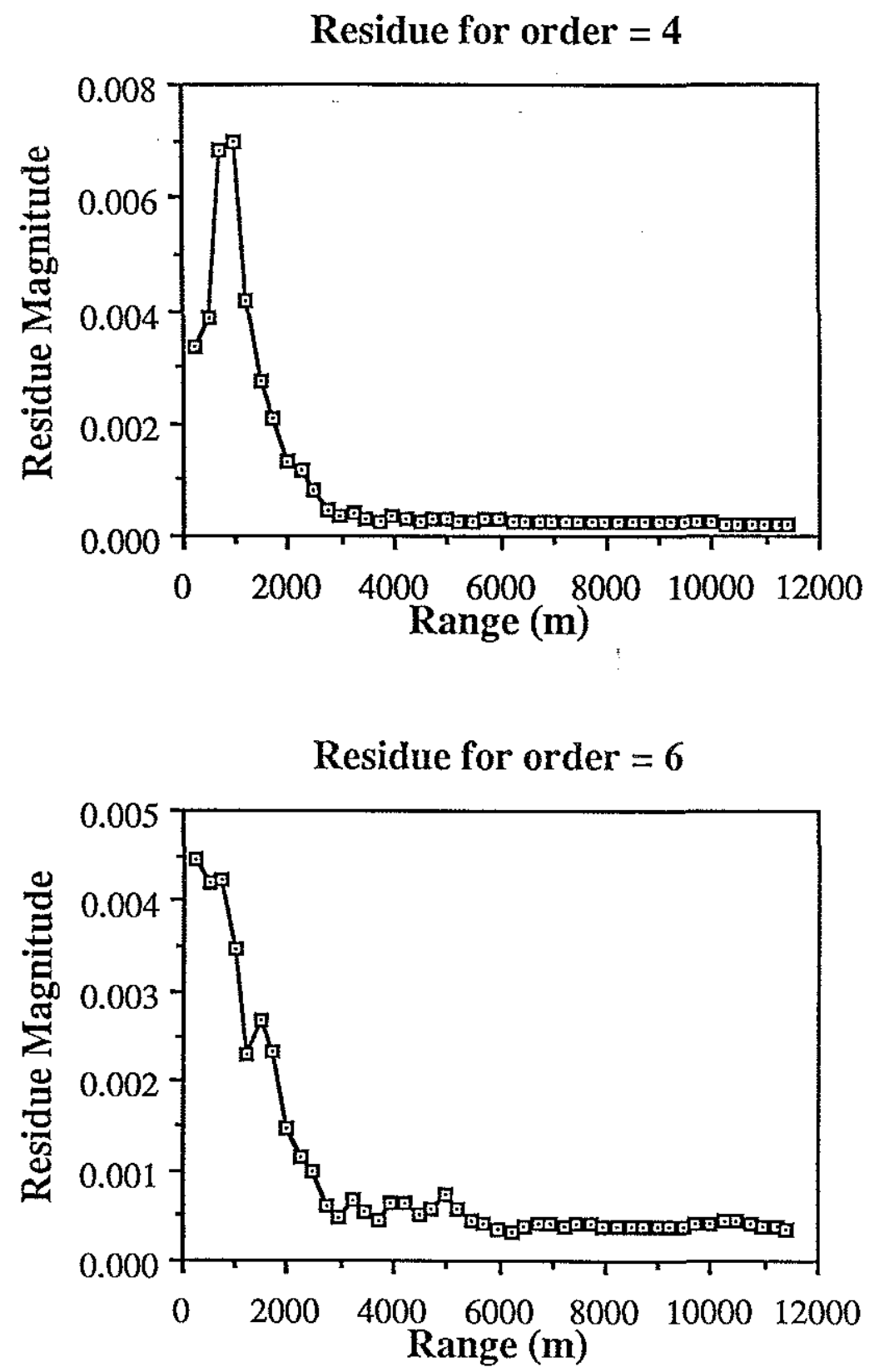

Fig 4.3.4 Residue graphs for step change model orders 4 and 6 

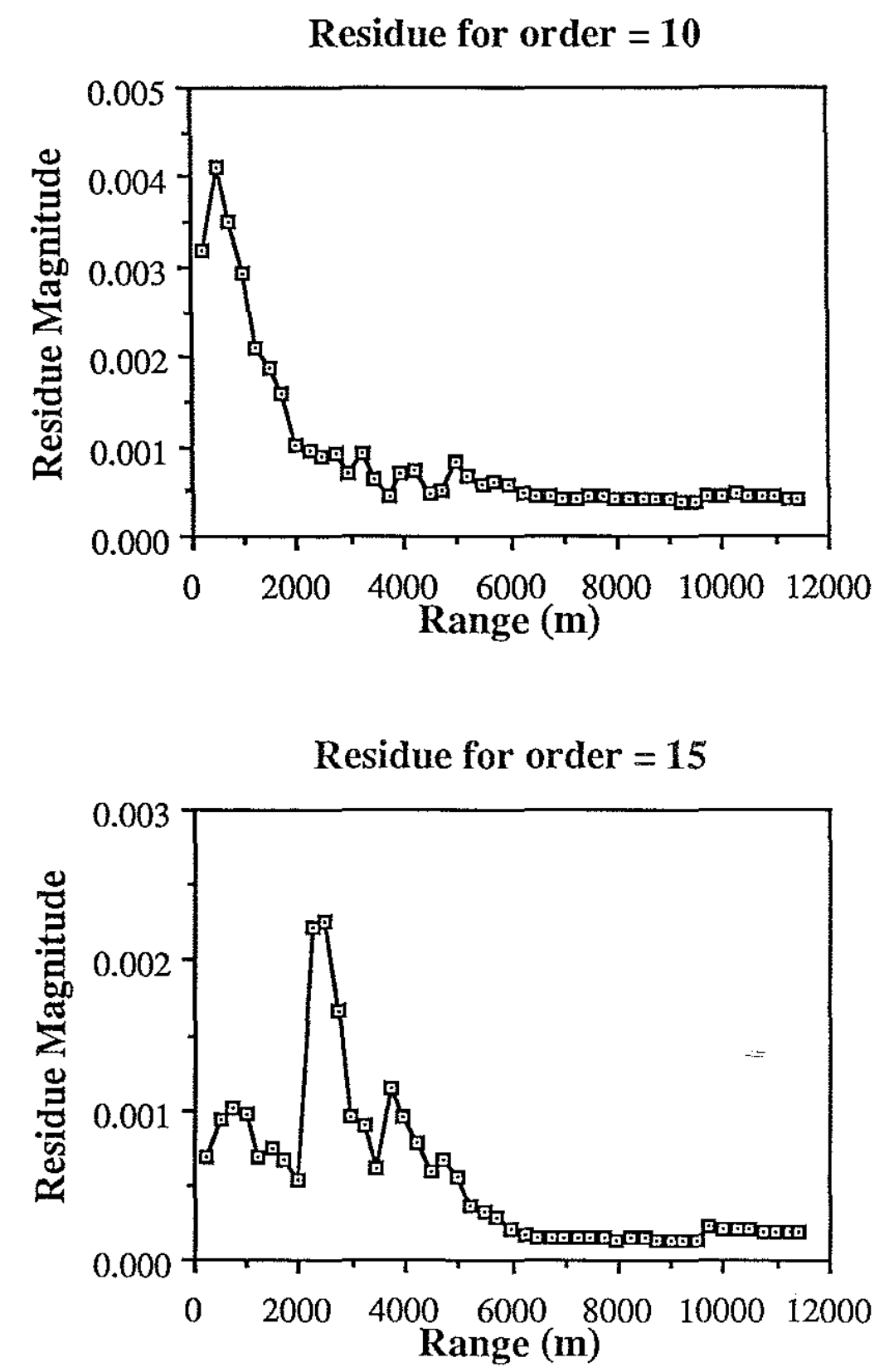

Fig 4.3.5 Residue graphs for step change model orders 10 and 15

The total residue (the sum of the individual residues for each range block) found in figure 4.3.6 shows the general trend of the decrease of total residue with increasing model order. The increase in total residue from an order 15 model to an order 18 model is due to the residue spikes in the higher order model. The 18 order model residue does, however, have a lower value than order 10 and most earlier models. 


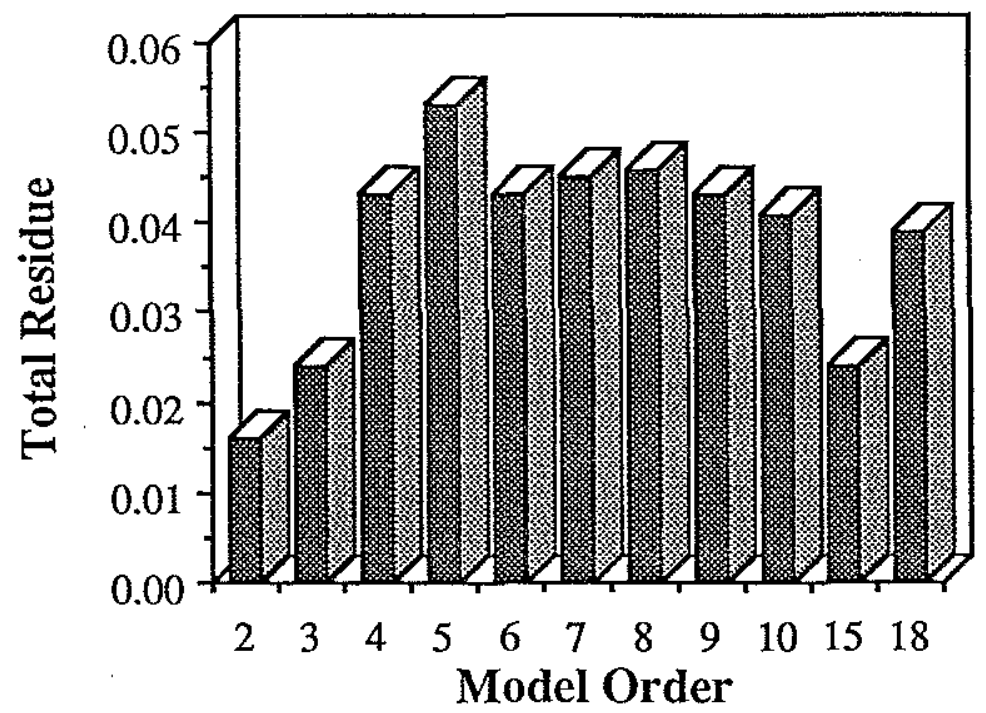

Fig 4.3.6 Total residues for step change case (50 pt, $5 \mathrm{~m}$ spacing, 0 overlap)

The low residues in the situations where order equals two or three is misleading as

demonstrated in the residue graph of figure 4.3.7 and the corresponding wavenumber graph of figure 4.3.8. Although the residue does, in fact, indicate the observed data fits an all pole model, the accuracy of the parameters found by an exactly specified case is questionable.

The ESD of the process indicates there is still energy in the region of the cutoff mode as the range interval from cutoff increases. The ESD also clearly indicates the spectral spreading and dissipative nature of the cutoff mode. 


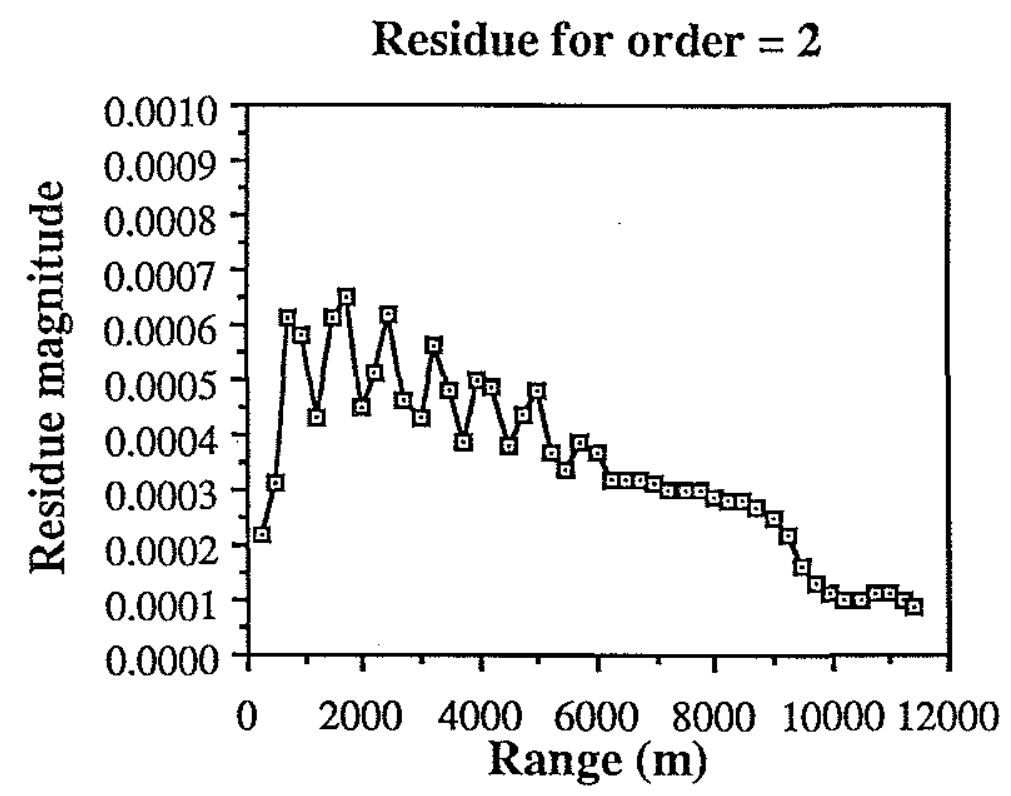

Fig 4.3.7 Residue vs. range for step change model order $=2$

(50 pt, $5 \mathrm{~m}$ spacing, 0 overlap)

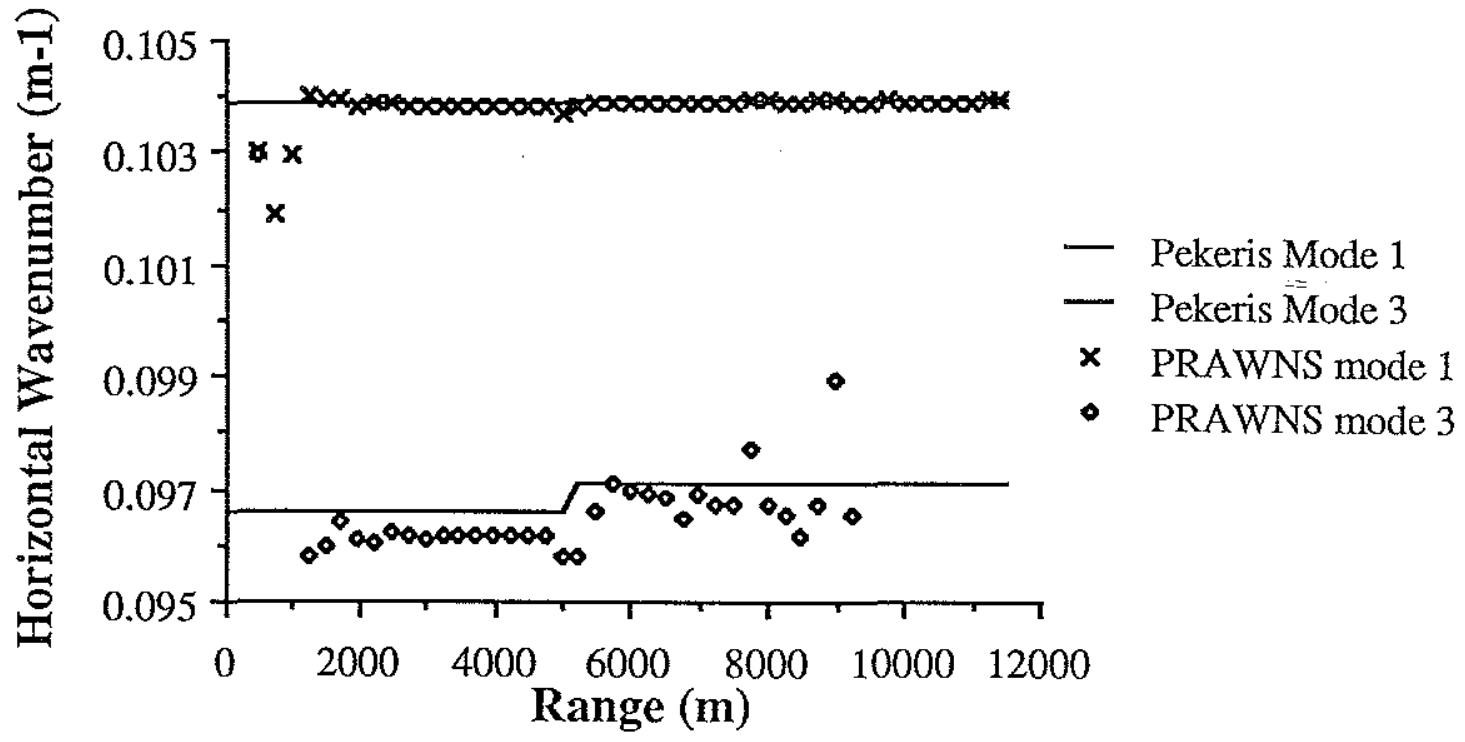

Fig 4.3.8 PRAWNS vs. Pekeris for step change model order $=2$ (50 pt, 5 m spacing, 0 overlap) 


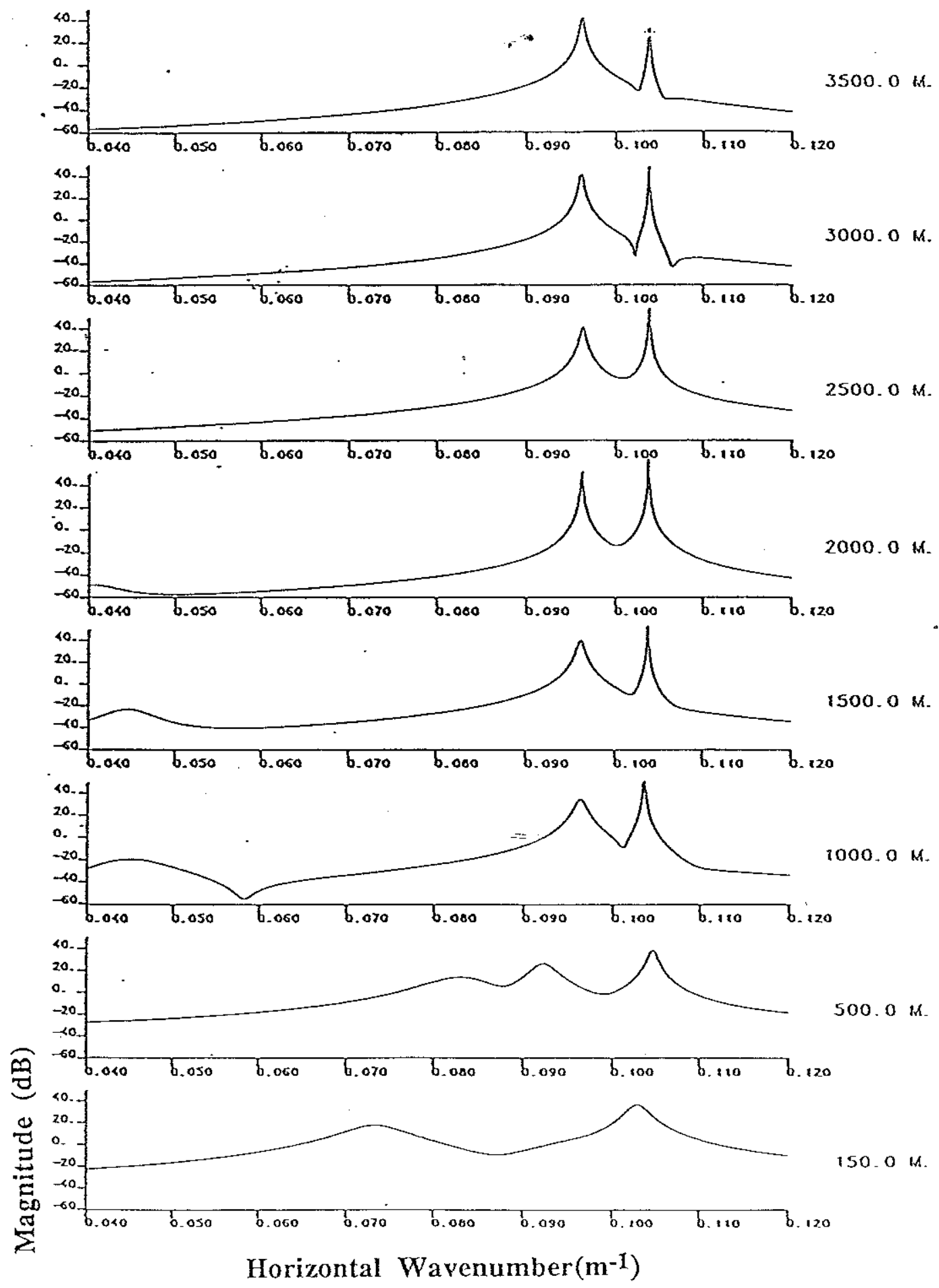

Fig 4.3.9 PRAWNS ESD for geoacoustic parameter shift example (50 pt, $5 \mathrm{~m}$ spacing, 0 overlap, model order 20 ) 


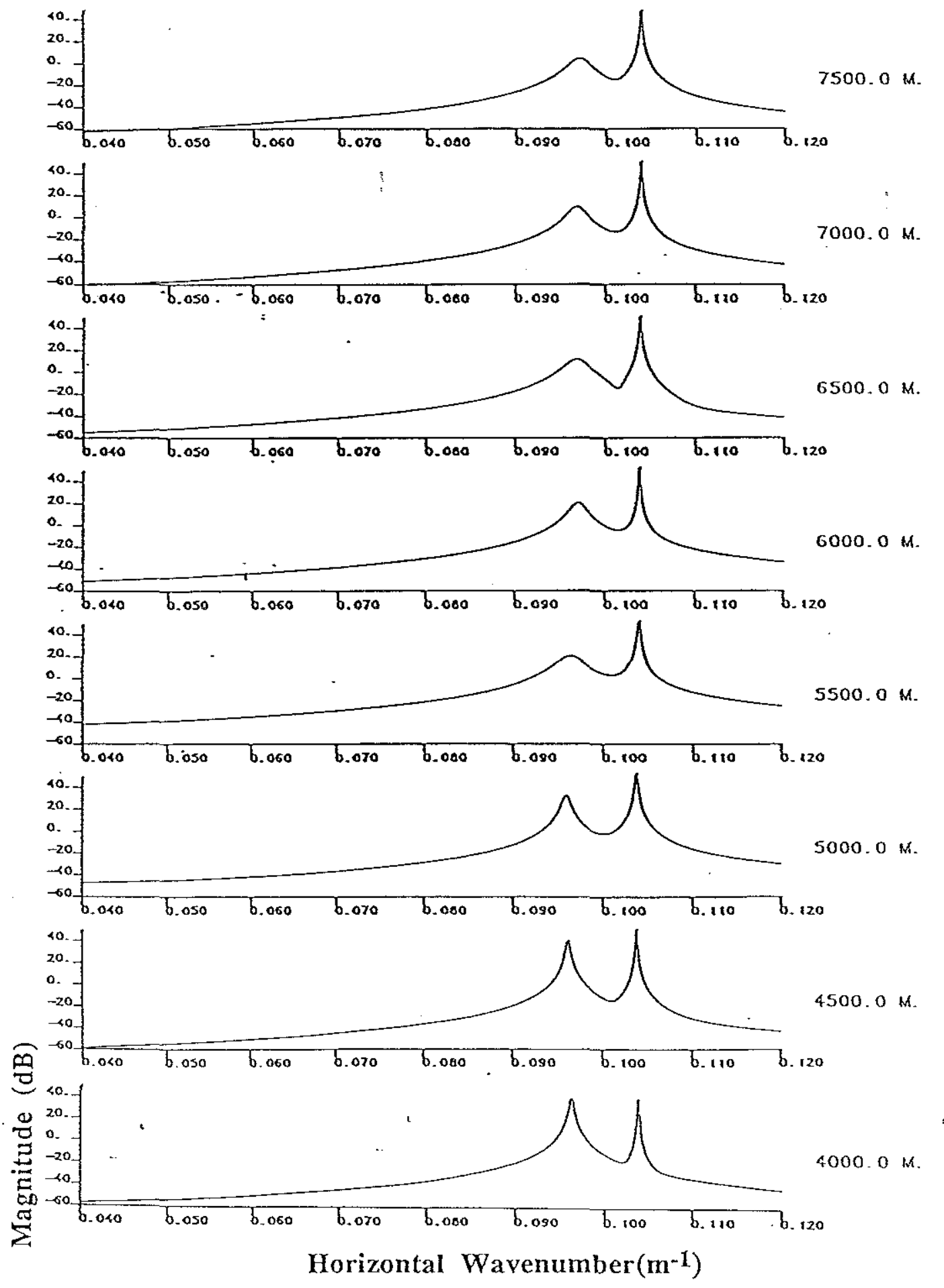

Fig 4.3.9(cont) PRAWNS ESD for geoacoustic parameter shift example (50 pt, $5 \mathrm{~m}$ spacing, 0 overlap, model order 20 ) 


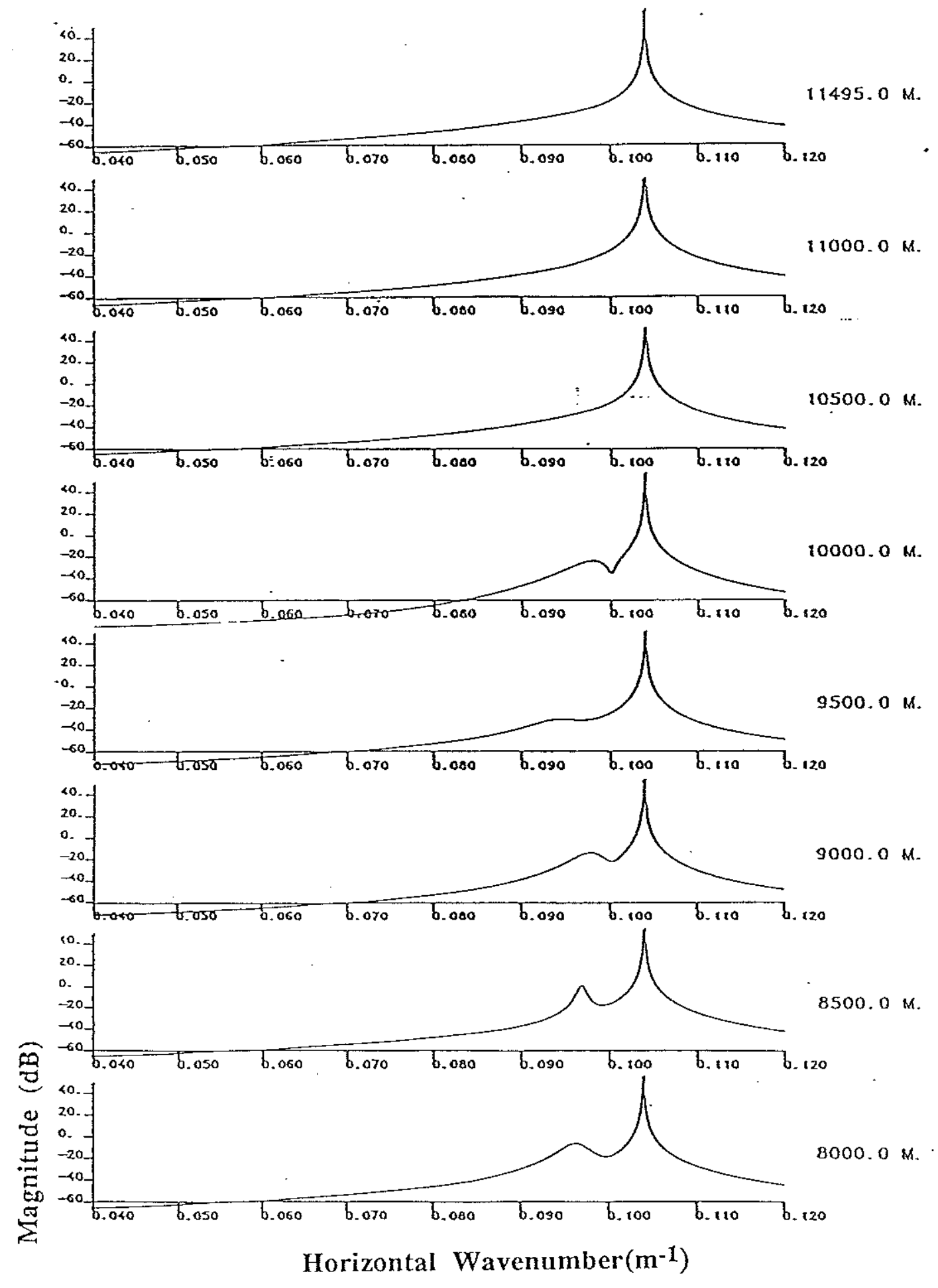

Fig 4.3.9 (cont) PRAWNS ESD for geoacoustic parameter shift example (50 pt, $5 \mathrm{~m}$ spacing, 0 overlap, model order 20 ) 
As a last confirmation of the algorithm's performance, the contour plot of the ESD for a variety of ranges in figure 4.3.10 shows the dissapation of mode 3 after the bottom transition at 5000 $\mathrm{m}$. The concentration of energy for both modes is narrow with an easily discernable peak.

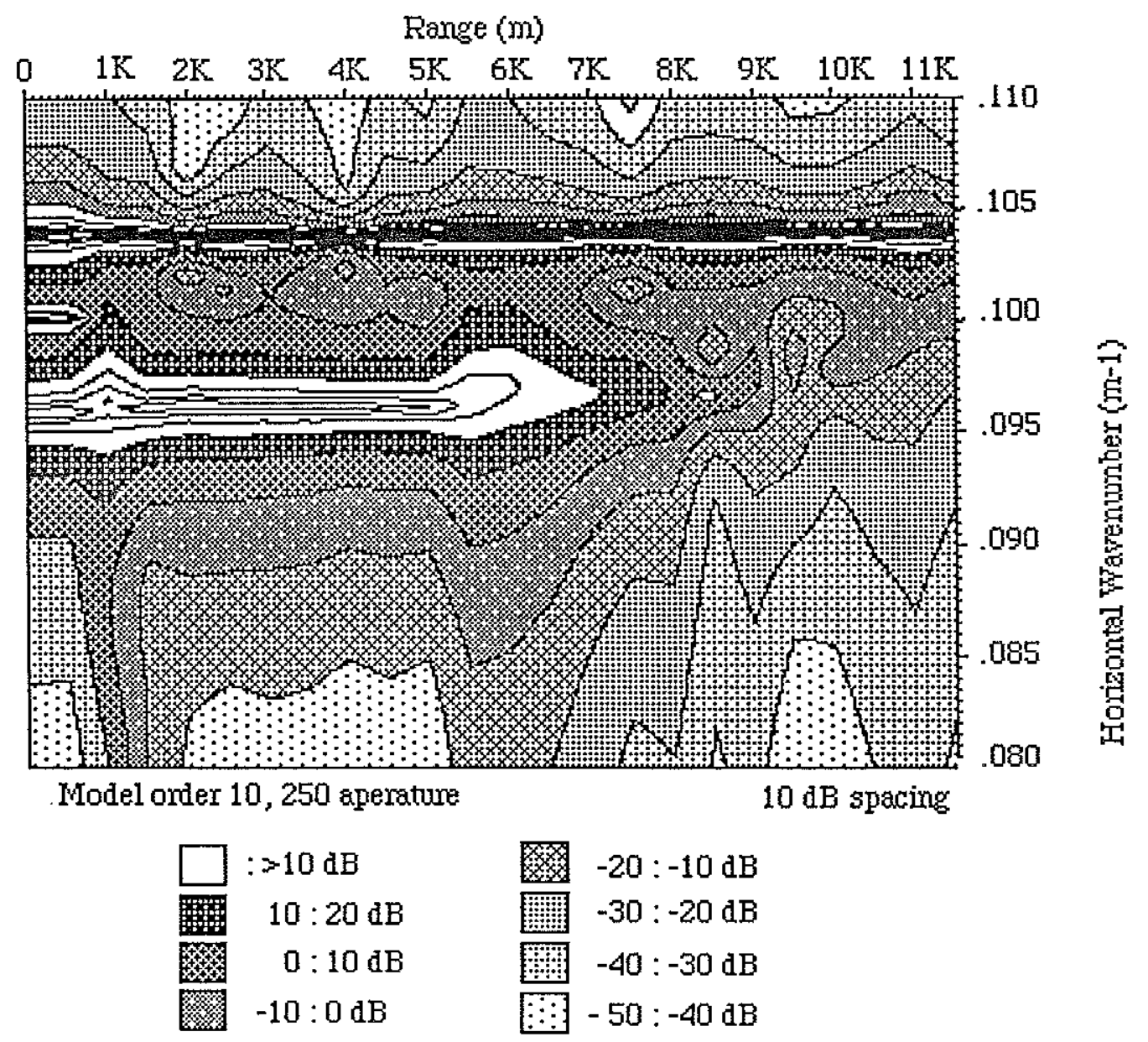

Fig 4.3.10 Contour plot of step change example ESD 


\subsection{Summary}

This chapter demonstrated the performance of Prony's method in a range dependent environment. The examples used consisted of fields generated by a parabolic equation algorithm (PAREQ) in waveguides which had bathymetry changes or bottom parameter shifts. The assumption of local adiabatic propagation was made; ie each section was considered to have locally invariant boundary conditions. The small apertures $(250 \mathrm{~m})$ proved more than sufficient for accurate determination of waveguide parameters.

While the determination of the residue allowed a quantitative assessment of the parameter choice, the residue is not recommended as a primary or exclusive evaluation method. At model orders which are the exact or close to the exact system order, the residue indicated an excellent fit to an all pole filter while the estimated parmeters were inaccurate when compared with the reference values (Pekeris waveguide). The ESD proved a more useful tool in this situation; the ESD also uses all estimated model parameters. 


\subsection{Recommendations and Future Considerations}

This study has exercised a specific algorithm designed for estimating modal wavenumbers, amplitudes, and attenuations to identify the strengths and shortcomings of this approach. In this section, we will briefly discuss areas which have been identified as requiring more attention and effort. Each of these areas deserves a separate study and should be considered for future successful employment of Prony's method. One such envisioned application of the high resolution technique is as a tool by which measured data may be manipulated to yield wavenumber estimates of propagating modes. These estimates can act as input for a perturbative scheme to bottom profile determination. Effectively, this treats the water column as a measurable medium which allows determination of the geoacoustic bottom parameters by recognizing the modal structure of the waveguide as a sampling mechanism.

Noise performance is the major obstacle in implementing this bottom evaluation scheme. The current algorithm's noise threshold of $30 \mathrm{~dB}$ SNR requires restructuring of the approach and, possibly, the experimental setup. One experimental change which would aid in processing the data would be the use of multiple data runs at the same site. This would permit an ensemble of data for processing rather than the "single snapshot" data series available in the current setup. Within the confines of the current experiment, each receiver was treated separately. A more sophisticated approach would correlate the data received by the two hydrophones. Care is required in associating the data from the two receivers; the different depth placement of the "vertical array" elements equates to different source receiver geometry. The field at each receiver will be influenced by the local sound velocity profile, source-receiver geometry and frequency. There are steps which should be investigated which may improve performance of the algorithm without changes in the experimental setup. These efforts may be broadly divided into pre-PRAWNS data processing and changes in the PRAWNS algorithm itself. 
One pre-PRAWNS processing approach which has met with limited success is a $\mathrm{k}_{\mathrm{r}}$ domain bandpass filter. In this scenario, the data is numerically Hankel transformed, bandpass filtered about user specified wavenumbers and inverse Hankel transformed back to a new pressure field. The ensuing pressure field is used as input to the Prony algorithm above. The bandpass filter used in the study was wider than the wavenumber section of interest since a specific window was not applied to the data.

To examine the effects of such filtering, we consider the Nantucket profile, with a SAFARI generated pressure field again. Without the bandpass filtering, the modal structure is as shown in the ESD plots of figure 5.1.1. If the same pressure field is first notched filtered in the $\mathrm{k}_{\mathrm{r}}$ domain and all PRAWNS inputs kept the same as the earlier plots, the ESD plot of figure 5.1.2 is obtained. Notice the "outlier" peaks of energy at the bandpass wavenumbers. While these outliers are distracting, they are due to the particular filter implimentation used. The wavenumbers of the three propagating modes is the same in each case. A better filtering scheme would use a finite impulse response (FIR) or smoothing filter on the pressure data[51].

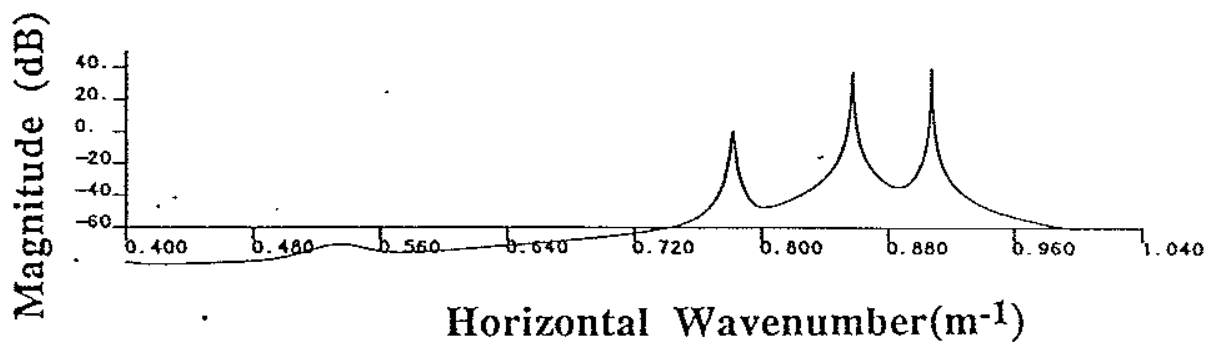

Fig 5.1.1 PRAWNS ESD for unfiltered $220 \mathrm{~Hz}$ Nantucket pressure field (SAFARI field, $100 \mathrm{pt}, 50 \mathrm{pt}$ overlap, $0.64 \mathrm{~m}$ spacing, model order 15) 


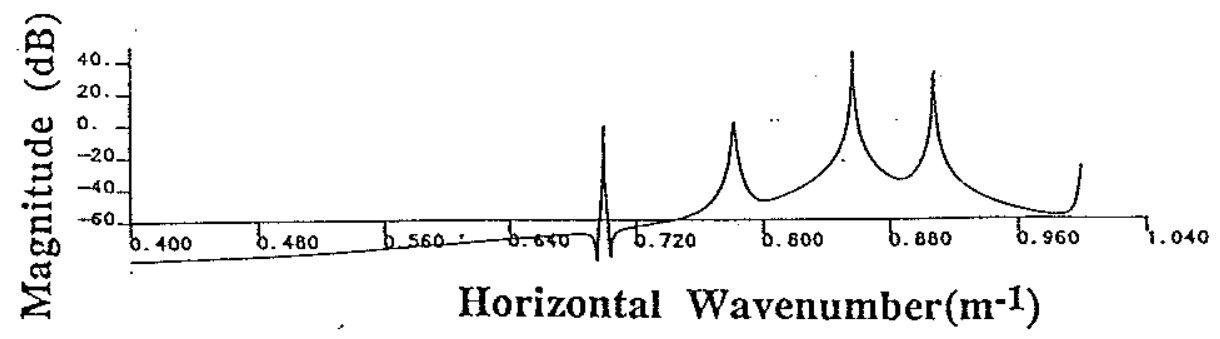

Fig 5.1.2 PRAWNS ESD for bandpass filtered $220 \mathrm{~Hz}$ Nantucket pressure field

(SAFARI field,100 pt, $50 \mathrm{pt}$ overlap, $0.64 \mathrm{~m}$ spacing, model order 15) (Passband from 0.7 to $1.0 \mathrm{~m}^{-1}$ )

The merits of this crude filtering are evident when the technique is used on real data. Consider the ESD of the Nantucket field experiment of chapter 3 (figures 3.4 .8 through 3.4.11). The low SNR (the Green's function of figure 3.4.6 has modal peaks roughly $6 \mathrm{~dB}$ above background) resulted in only one PRAWNS mode identification in each case. Figures 5.1.3 through 5.1.6 illustrate the effects of bandpass filtering on the pressure fields of actual data. The ESDs of these figures were generated using the same PRAWNS inputs as the non filtered versions of chapter 3 . The outlier peaks are present; the $140 \mathrm{~Hz}$ field had a passband of 0.4 to $0.8 \mathrm{~m}^{-1}$ while the $220 \mathrm{~Hz}$ field passband was 0.7 to $1.0 \mathrm{~m}^{-1}$. Notice the additional mode(s) found in the ESD. Wavenumber plots of the modes identified show reasonable agreement between both hydrophones and, apparently, a correlation with the bathymetry (figures 5.1.7 through 5.1.9). Table 5.1.1 lists the wavenumbers of the peak values of the Green's function over the entire aperture. The wavenumbers are shown as "reference" wavenumbers in figures 5.1.7 and 5.1.8; actually, the numerical Hankel transform used has problems. The difficulty is that the Hankel transform assumes boundary condition invariance over the interval of interest. Changes in bathymetry or bottom properties will change the parameter estimates; however, as boundary conditions become complex, the shift in parameter estimates for a given boundary condition variation becomes less predictable. The PRAWNs approach also makes the adiabatic approximation; the smaller aperture of this method makes 
the validity of the assumption more likely. Future improvements in this approach include a more sophisticated filtering scheme to improve mode resolution and to eliminate the bandpass wavenumber "outlier" spikes.

Changing the algorithm used may also improve performance in noise. A prime candidate for enhancing the current method is the SVD based approach espoused by Tufts and Kumaresan[44,45]. The method adjusts the matrix used to determine filter coefficients by evaluating the matrix for breakpoints in the singular values. This may require the assumption of no damping (ie, real eigenvalues) and then solving for damping by other means such as the consecutive block scheme of chapter 2.

The alogrithm may be altered to use a modified Prony's method with an autocorrelation or autocovariance matrix used in place of the signal matrix. Use of these matrices have not yet been explored in depth for this application and may prove more robust in noise than the present approach. Another method used by researchers seeking a stable filter with robust noise performance is the forward backward linear predictor (FBLP)[31]. The FBLP assumes real eigenvalues to satisfy the stationary attributes of the signal; the signal should look the same in the forward or backward directions. This can not strictly be satisfied by a decaying exponential. If no attenuation is assumed to occur, this method might be used to identify the wavenumbers and initial phase for the propagating modes. This information could then be used in the current algorithm to reduce the order of the problem by, in effect, factoring the known information from the polynomial to be rooted[31]. Similarly, the incorporation of the PRAWNS algorithm into a perturbative or iterative scheme to correct parameter estimates may provide a more robust method. Throughout the exploration of alternate methods, maintaining the short apertures of the approach studied here must be emphasized. The advantage of a high resolution scheme and parameter model is that it allows a priori knowledge of the acoustic propagation to be used to shape the model. This allows short data sets to be used which are 


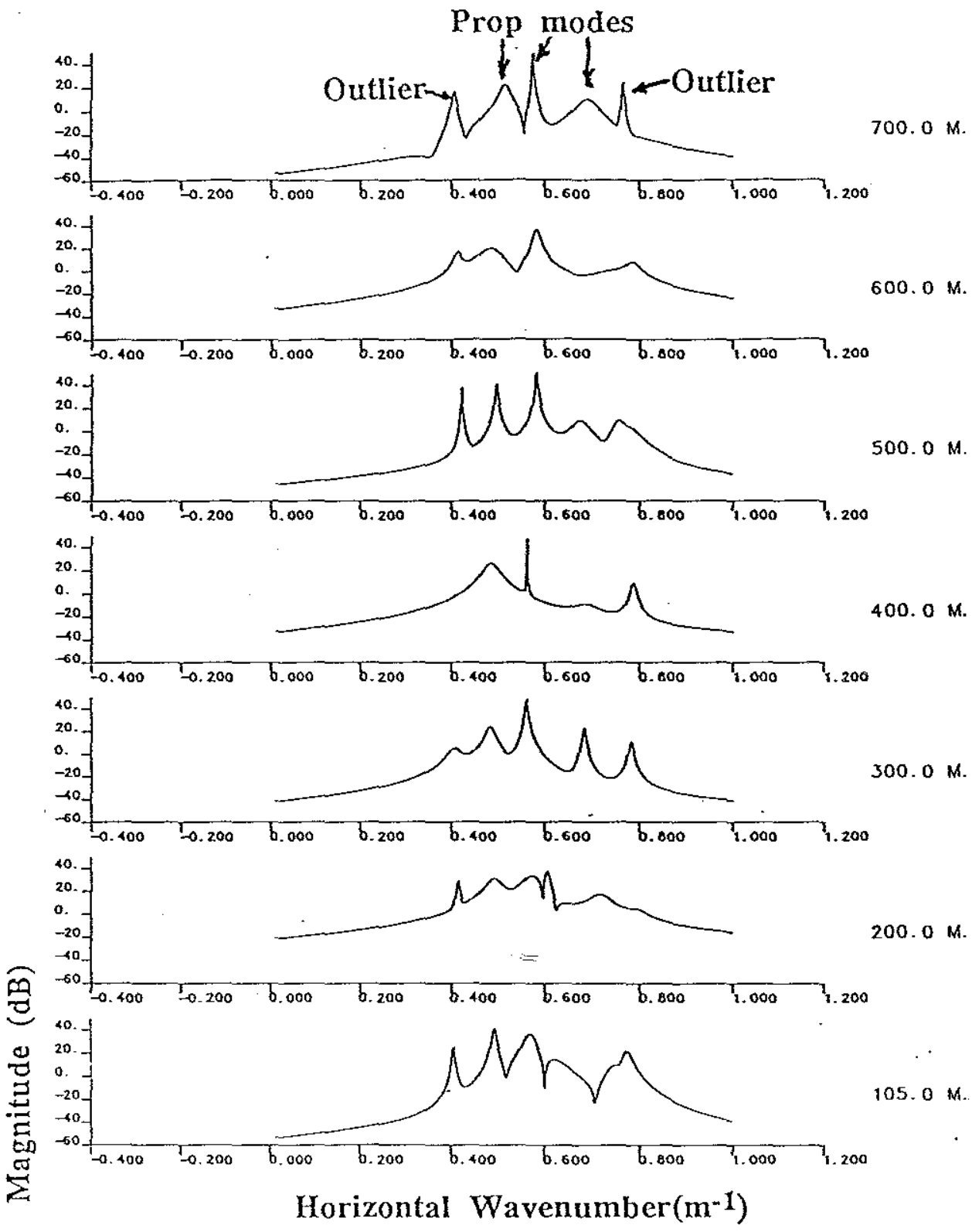

Fig 5.1.3 PRAWNS output of BP filtered Nantucket $140 \mathrm{~Hz}$, upper hyd. (100 pt, 50 pt overlap, $0.64 \mathrm{~m}$ spacing, model order 20 ) 

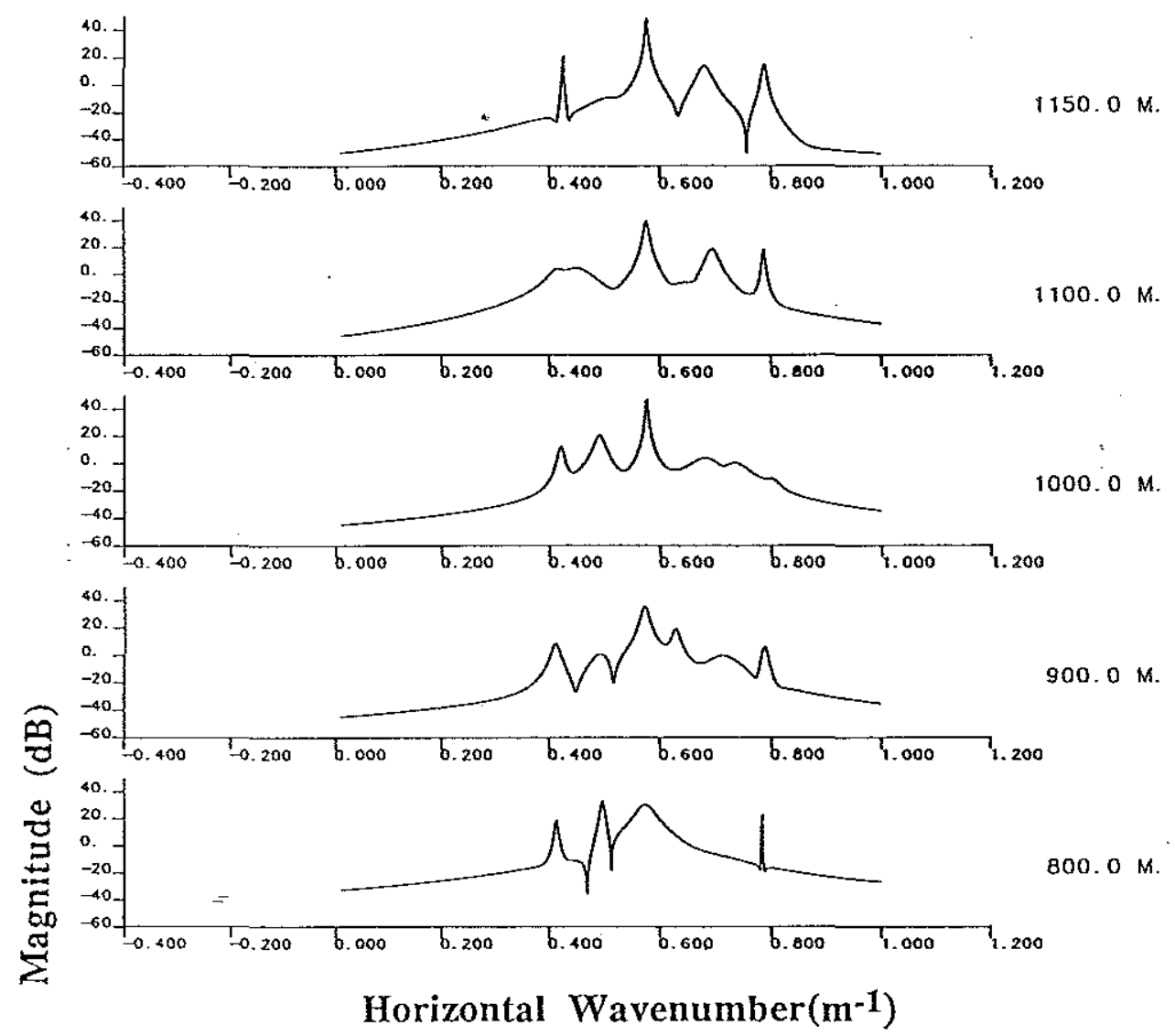

Fig 5.1.3(cont) PRAWNS output of BP filtered Nantucket $140 \mathrm{~Hz}$, upper hydrophone (100 pt, $50 \mathrm{pt}$ overlap, $0.64 \mathrm{~m}$ spacing, model order 20 ) 


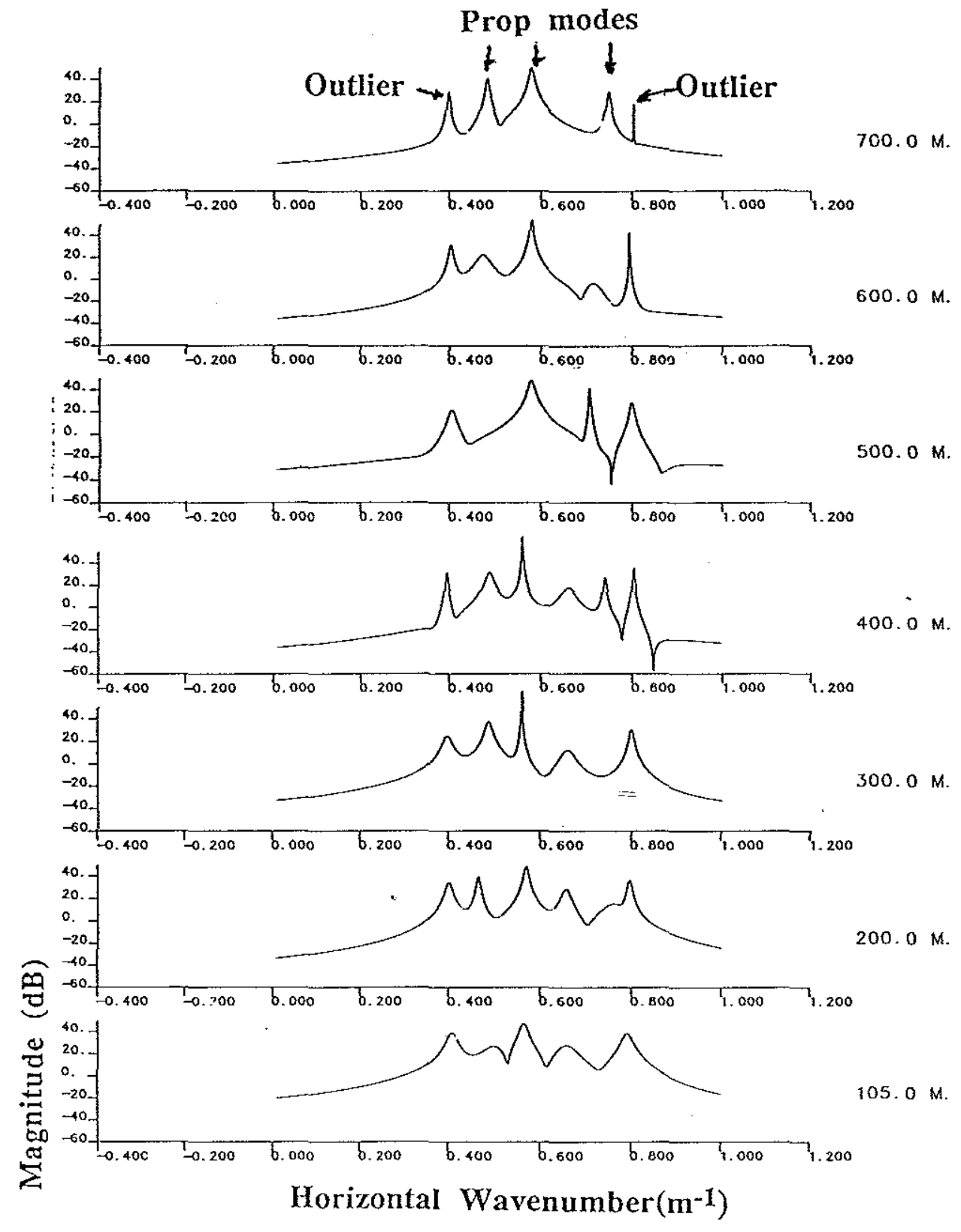

Fig 5.1.4 PRAWNS output of BP filtered Nantucket $140 \mathrm{~Hz}$, lower hyd. (100 pt, $50 \mathrm{pt}$ overlap, $0.64 \mathrm{~m}$ spacing, model order 20) 


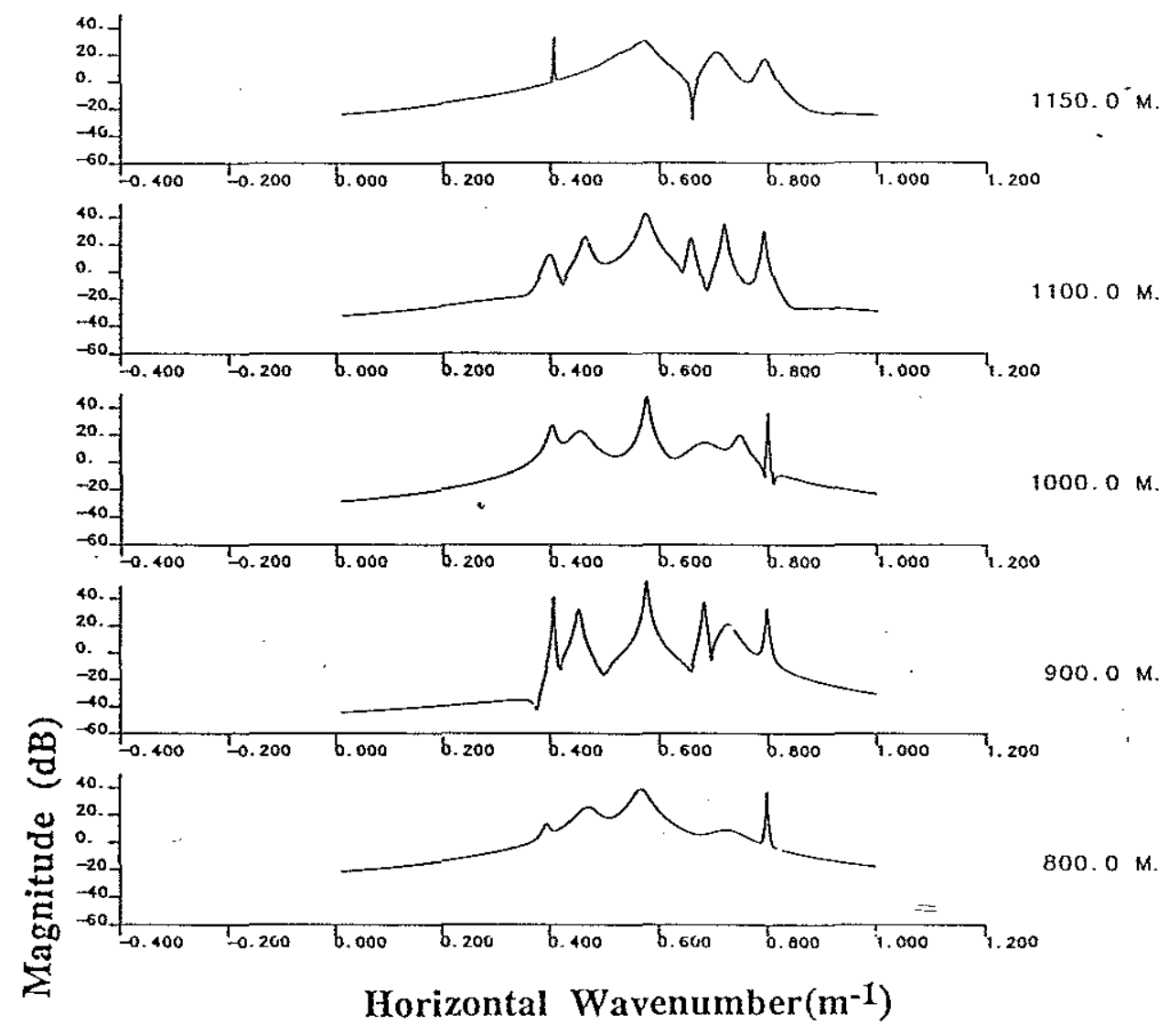

Fig 5.1.4 (cont) PRAWNS output of BP filtered Nantucket $140 \mathrm{~Hz}$, lower hydrophone

(100 pt, 50 pt overlap, $0.64 \mathrm{~m}$ spacing, model order 20) 


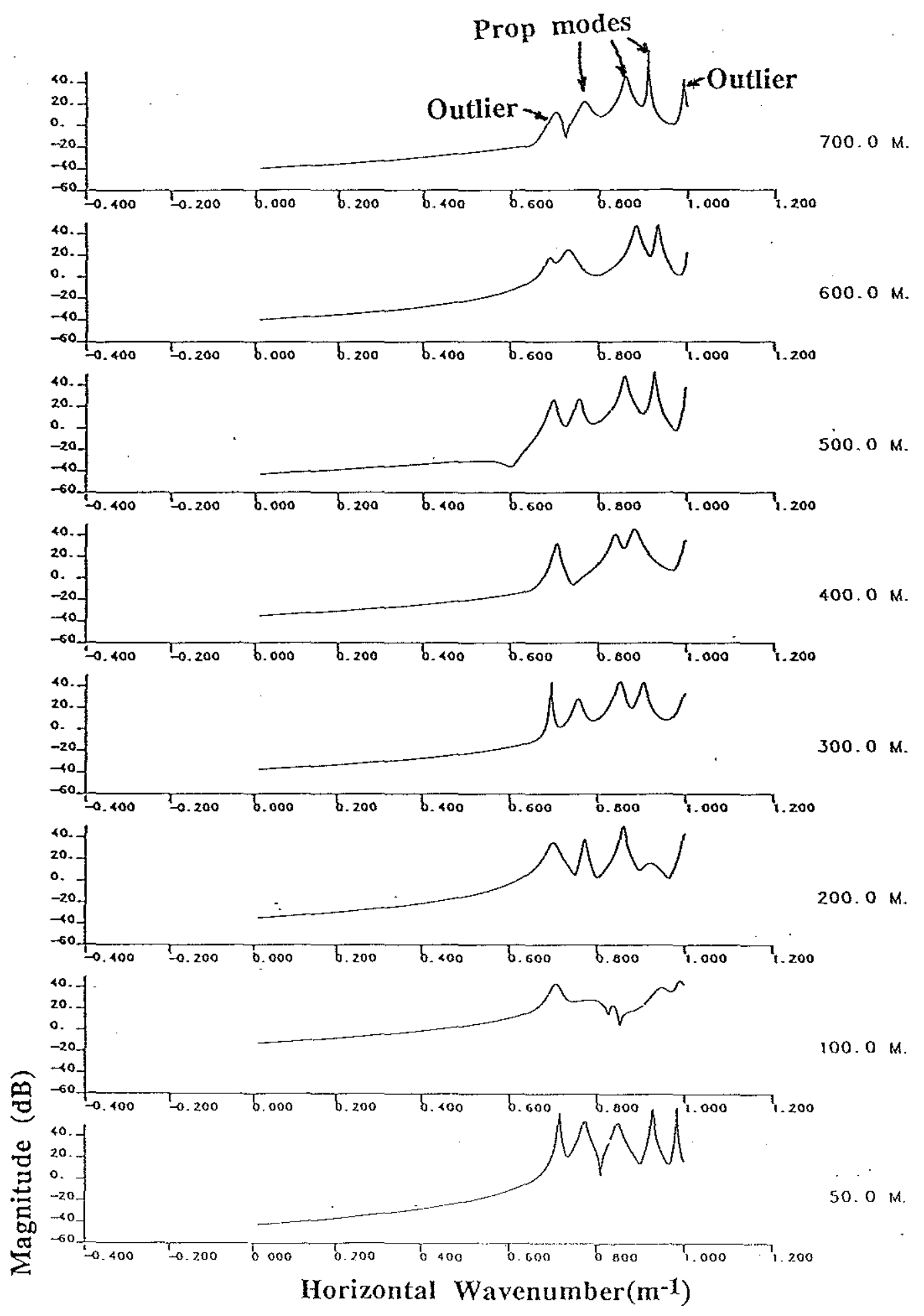

Fig 5.1.5 PRAWNS output of BP filtered Nantucket $140 \mathrm{~Hz}$, upper hyd $(100 \mathrm{pt}, 50 \mathrm{pt}$ overlap, $0.64 \mathrm{~m}$ spacing, model order 20 ) 


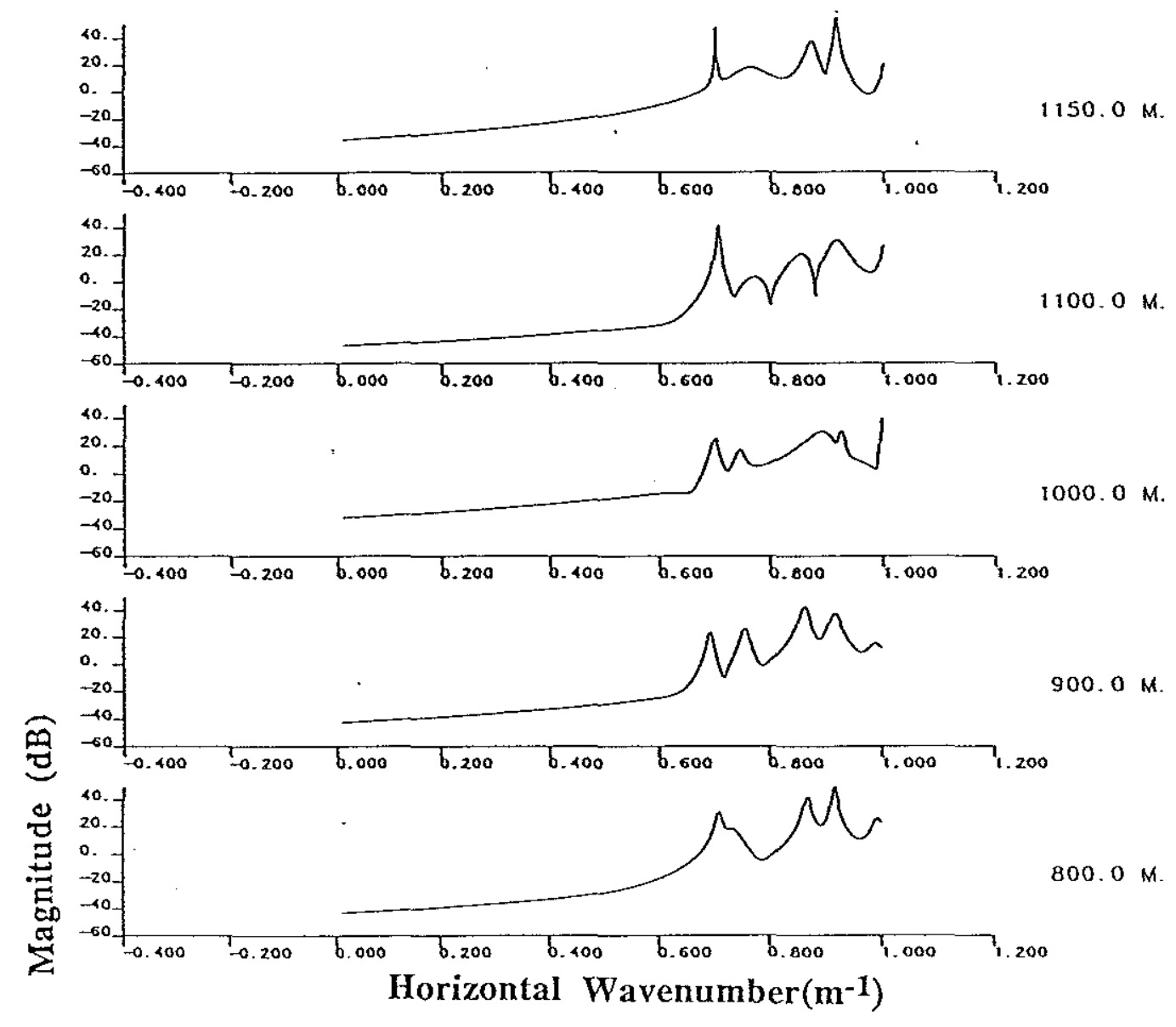

Fig 5.1.5(cont) PRAWNS output of BP filtered Nantucket $140 \mathrm{~Hz}$, upper hyd (100 pt, $50 \mathrm{pt}$ overlap, $0.64 \mathrm{~m}$ spacing, model order 20) 


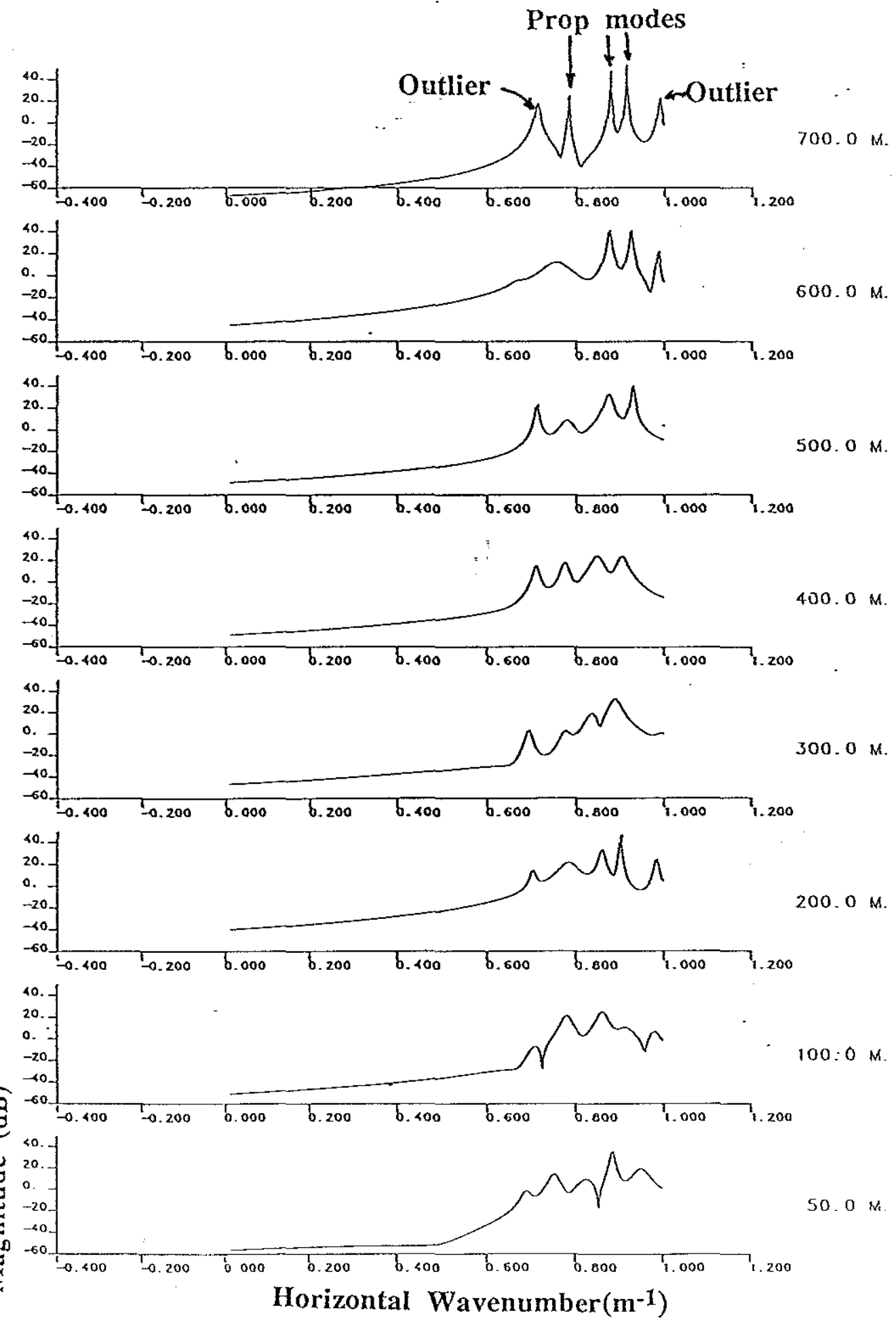

Fig 5.1.6 PRAWNS output of BP filtered Nantucket $140 \mathrm{~Hz}$, lower hyd (100 pt, $50 \mathrm{pt}$ overlap, $0.64 \mathrm{~m}$ spacing, model order 20) 


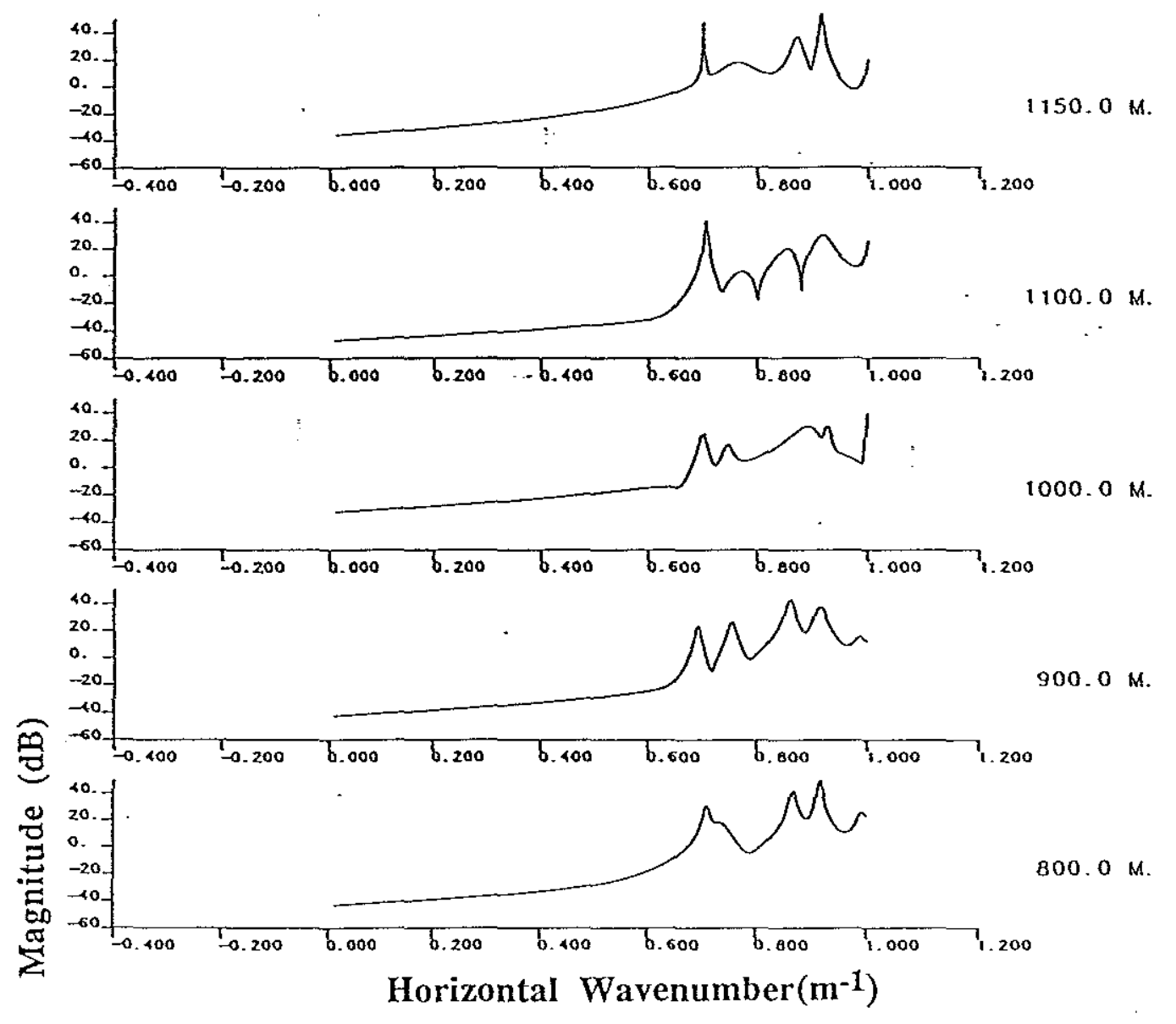

Fig 5.1.6(cont) PRAWNS output of BP filtered Nantucket $140 \mathrm{~Hz}$, lower hydrophone

(100 pt, $50 \mathrm{pt}$ overlap, $0.64 \mathrm{~m}$ spacing, model order 20 ) 


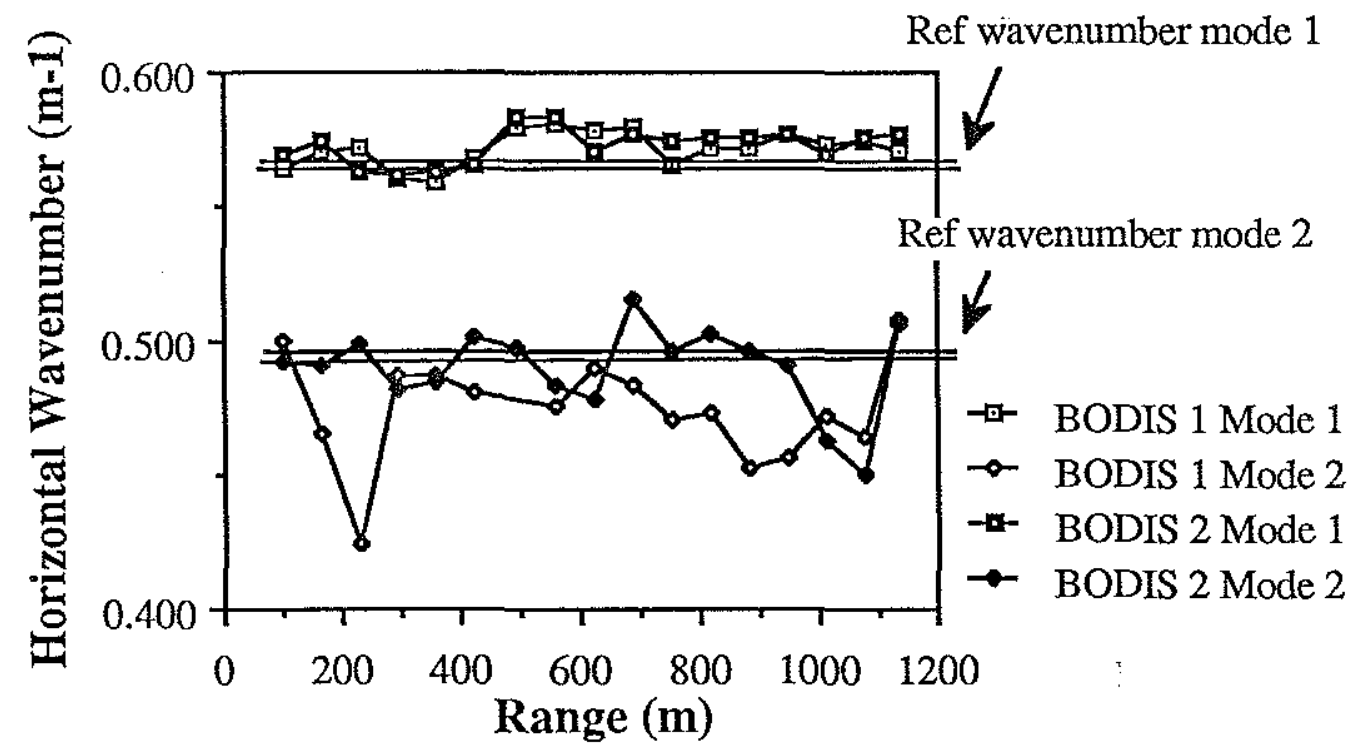

Fig 5.1.7 Wavenumber vs. range for $140 \mathrm{~Hz}$ Nantucket data (100 pt, $50 \mathrm{pt}$ overlap, $0.64 \mathrm{~m}$ spacing, model order 20 )

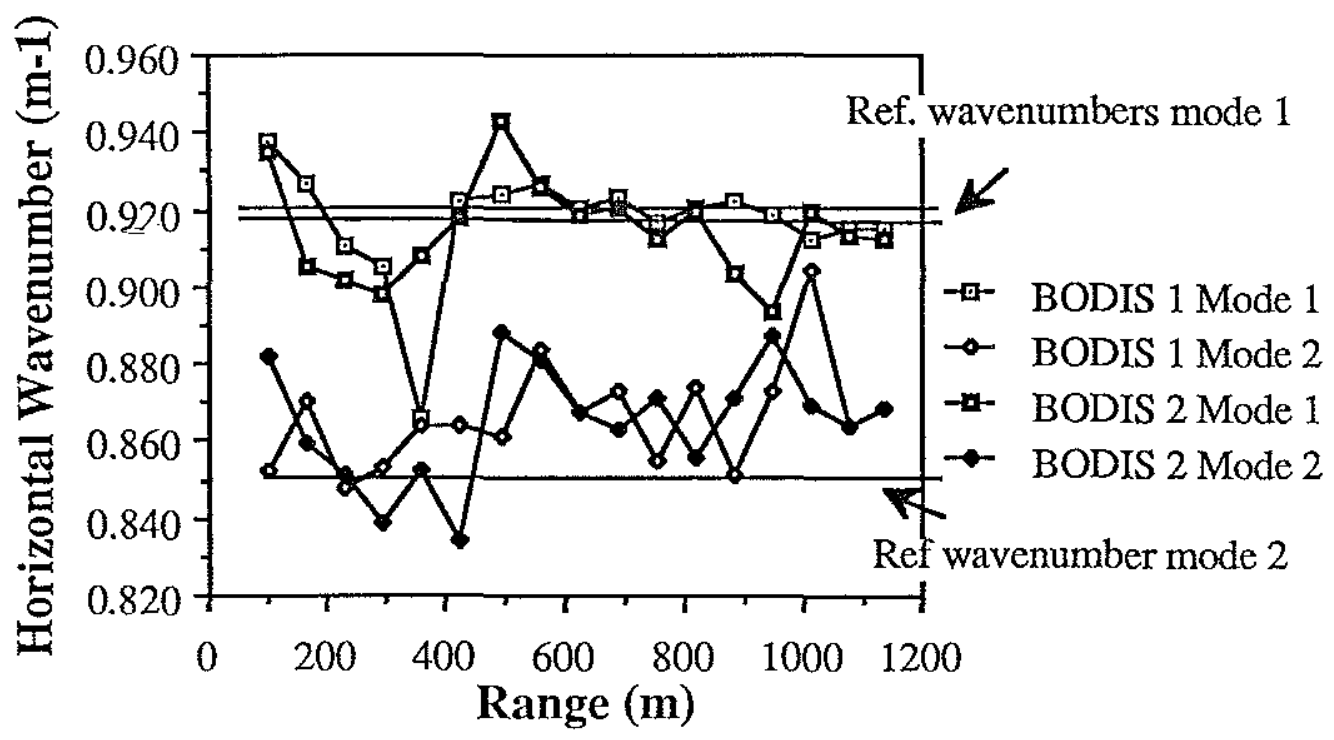

Fig 5.1.8 Wavenumber vs. range for $220 \mathrm{~Hz}$ Nantucket data (100 pt, $50 \mathrm{pt}$ overlap, $0.64 \mathrm{~m}$ spacing, model order 20 ) 


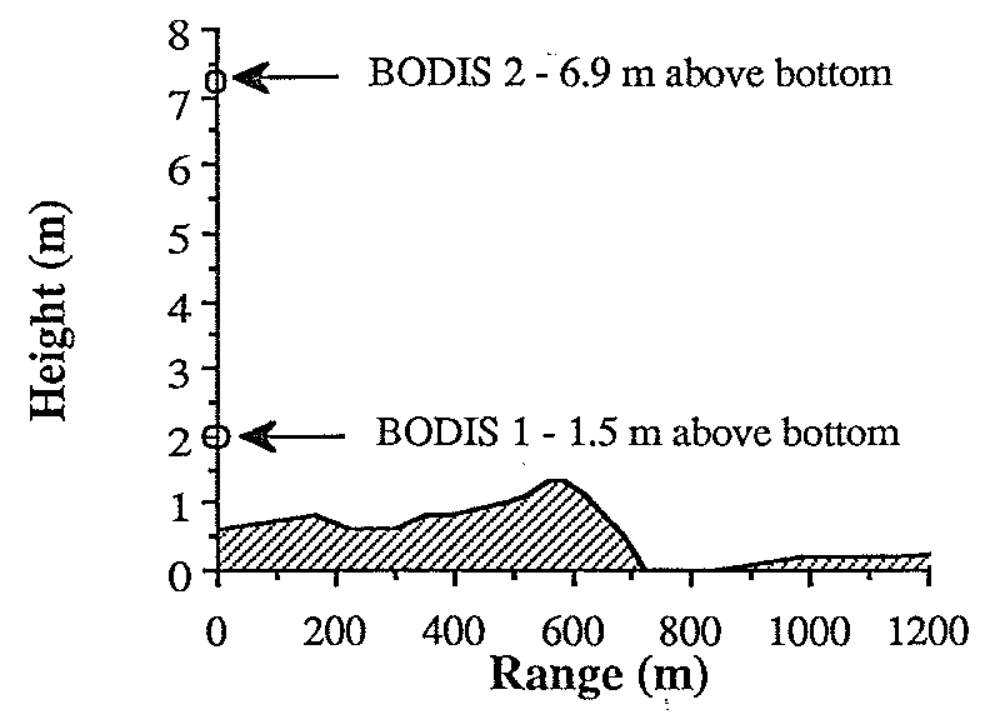

Fig 5.1.9 Nantucket bottom bathymetry

\begin{tabular}{|cccc|}
\hline \multirow{2}{140\mathrm{Hz}}{} & Mode 1 & 0.5637019 & 0.5670657 \\
& Mode 2 & 0.4955879 & 0.4935128 \\
& & & \\
$220 \mathrm{~Hz}$ & Mode 1 & 0.9207131 & 0.9182185 \\
& Mode 2 & 0.8502503 & 0.8494114 \\
\hline
\end{tabular}

Table 5.1.1 Reference wavenumber values for Nantucket data (From peak values of Green's function over entire data set)

essential in the range dependent waveguides found in field experiments. Large apertures may improve performance in noise but the cost of using additional data must not be taken lightly.

While the incorporation of a noise reduction scheme is necessary, there are other areas which should be addressed in future work. The incorporation of an analytic method to determine model order would greatly ease the work of an experimenter. An SVD scheme 
similar to that of Braun and Ram warrants investigation. One particular feature of the PRAWNS code which was not exploited was the theoretical ability to detect a reflected wave. This was beyond the scope of this initial study. Once a reliable means of generating such data is identified, the results should provide more information on new algorithms to test.

Additionally, the near field environment was not addressed in this study. 


\subsection{Conclusions}

The Prony model has been shown to fit the far field modal structure of a shallow water waveguide quite well. The implementation of this parameter estimation aproach has several important implications.

With this technique, the experimenter has a fast, efficient tool to use to examine the modal structure of a waveguide. The parameter estimates may be manipulated and transformed to provide energy information as well as tabular values. The four tools presented in this study to assist the researcher include the energy spectral density (ESD), wavenumber, residue and pole plots. The advantage of each was outlined in chapter 3. The ESD essentially provides an overall summary of propagating and virtual modes. The wavenumber and residue plots are used in an iterative scheme to obtain a good model order. Pole plots are used to identify propagating modes among the arbitrary modes.

A parameter estimation model directly generates desired properties of the sound energy field. Other methods, such as a pertubative inverse solution to the bottom profile make use of these wavenumber estimates as inputs. Prony's method may replace less accurate methods, which were required in the past, such as peak searching routines with short aperture Hankel transforms, to find these values. Additionally, properties such as the attenuation are directly accessible as a result of the use of the Prony fit.

The use of a high resolution approach permits use of a short range aperature which allows exploration of range dependent features. Chapter 4 presented examples of shifts in bathymetry and bottom properties. The ability to track range dependent waveguide aspects is available through the adiabatic propagation assumption; the waveguide boundary conditions are assumed to be invariant over the local (processing block) range of interest.

A major shortcoming of the current algorithm is its sensitivity to noise. For SNR below $30 \mathrm{~dB}$, estimation of model parameters tended to be both biased and to have a large 
variance; ie the estimates were inaccurate. Further exploration is necessary to formulate a more robust algorithm for use in a field environment.

The search for analysis techniques for studying the ocean environment is continuing to generate new approaches to old problems. While the approach first postulated by Gaspard Riche in 1795 hardly ranks as a new concept, this application has the effect of a fresh look at the shallow water environment. Continued development and improvement of such high resolution techniques should provide the researcher with a formidable tool indeed. 


\section{Appendix A. Essentials of Sturm Liouville Problems}

\section{Sturm Liouville Problem}

If a given, second order, differential equation may be cast in the form:

$$
\frac{d}{d x}\left[m(x) \frac{d y}{d x}\right]+[q(x)+\beta r(x)] y=0
$$

with:

- $m, q, r$ real and continuous over the interval[a,b]

- $\beta=$ separation constant

$\cdot r=$ weighting coefficient

- homogeneous boundary conditions at $x=a, b$ of

$$
\begin{aligned}
& A \frac{d y(a)}{d x}+B y(a)=0 \\
& C \frac{d y(b)}{d x}+D y(b)=0
\end{aligned}
$$

then the problem is known as a Sturm Liouville problem[15]. The solution to the boundary value problem has eigenvalues which are real and non-negative. In addition the eigenfunctions associated with the eigenvalues form an orthonormal set which is complete. The implications of this characteristic include:

- the eigenfunctions, $\phi(x)$, are unique to within a multiplicative constant

- the eigenfunctions are orthonormal, ie. $\int_{a}^{b} r(x) \phi_{n}(x) \phi_{k}(x) d x=\delta_{n k}$

- any arbitrary function of $\mathrm{x}$ can be expressed as a weighted sum of eigenfunctions, ie. $f(x)=\sum_{n} c_{n} \phi_{n}(x)$ where $c_{n}=$ a coefficient of $f$ with respect to the orthonormal set $\{\phi\}$.

An inhomogeneous Sturm Liouville problem has the form (where $\Lambda=\beta$ ):

$$
\frac{d}{d x}\left[m \frac{d y}{d x}\right]+q y+\Lambda r y=f(x)
$$

with the homogeneous boundary conditions above satisfied. 
It has the solution:

$$
\sum_{n} \frac{A_{n} \phi_{n}}{\Lambda-\beta_{n}}
$$

in which:

- $\phi_{\mathrm{n}}$ are the eigenfunctions which satisfy the homogeneous equation (A.1) with eigenvalues $\beta_{\mathrm{n}}$.

- $A_{n}$ are found by using $\frac{f(x)}{r(x)}=a_{n} \phi_{n}(x)$ and exploiting the orthogonality property of $\phi_{n}$.

- $\Lambda$ is arbitrary and not influenced by boundary conditions. For the case of an inhomogeneous Sturm Liouville problem where $f(x)=\delta\left(x-x_{0}\right)$, the solution to the inhomogeneous equation is known as the Green's function and denoted $\mathrm{G}\left(\mathrm{x}, \mathrm{x}_{0}\right)$. This $\mathrm{G}\left(\mathrm{x}, \mathrm{x}_{0}\right)$ may be expanded in terms of eigenfunctions of the homogeneous equation. A last note before moving from this extremely cursory treatment of the subject area is that the Green's function may be shown to be symmetric so that $G\left(x, x_{0}\right)=G\left(x_{0}, x\right)$. The consequence of this symmetry is evident in the solution to the pressure field in the waveguide in which source and receiver may be interchanged with no change in the field distribution; this trait is commonly referred to as acoustic reciprocity. 


\section{Appendix B - Some Signal Processing Basics}

As is often the case in signal processing problems, the algorithms described in this paper use discrete samples. There are distinctions in the discrete sample environment from the continuous time(or spatial) case. In many cases, direct analogies may be made[51].

\section{Linear Constant Coefficient Difference Equations (LCCDE)}

Given a differential equation with linear constant coefficients of the form:

$$
\sum_{k} a_{k} \frac{d^{k} y}{d r^{k}}=\sum_{s} b_{s} \frac{d^{s} y}{d r^{s}}
$$

to specify an output, $y(r)$, homogeneous and particular solutions to the differential equation must be determined. Particular solutions are dependent on initial conditions (n conditions are required for an nth order system). If the system is causal (or non anticipatory) and linear, the inital conditions are equal to zero.

The discrete time (or, in this case, discrete space) analogy to the differential equations are difference equations. The difference equation used to describe the system is:

$$
\sum_{k=0}^{P} a_{k} y[n-k]=\sum_{s=0}^{M} b_{s} x[n-s]
$$

As in the case of the continuous time case, the difference equation system solution, $y[n]$, is

the sum of a homogeneous $\left(\sum_{k=0}^{P} a_{k} y[n-k]=0\right)$ and particular solution. The description of a discrete system by a LCCDE results in a rational system function. The homogeneous solution of the difference equation has the form:

$$
\mathrm{y}_{\mathrm{h}}[\mathrm{n}]=\sum_{\mathrm{k}=0}^{\mathrm{P}} \mathrm{A}_{\mathrm{k}} \mathrm{z}_{\mathrm{k}}^{\mathrm{n}}
$$

Signal processing literature and texts make reference to linear time invariant (LT) systems. A more accurate description is linear shift invariant (LSI) systems since the data may represent a spatial sampling. A shift invariant system has the following property: if an input $x[n]$ yields an output $y[n]$ then $x\left[n-n_{0}\right]$ will result in an output $y\left[n-n_{0}\right]$. For a LSI 
system, causality is met by the necessary and sufficient condition $h[n]=0$ for $n<0$. Physical systems are usually modelled as causal since the system output doesn't depend on the future value of the input (or, equivalently, the system is non anticipatory). The assumption of LSI is common; if the system is not, in fact, LSI, a given segment of the data is assumed LSI and the parameters are calculated for each section.

\section{Z Transforms}

The bilateral $\mathrm{z}$ transform is defined as:

$$
X(z)=\sum_{n=-\infty}^{\infty} x[n] z^{-n}
$$

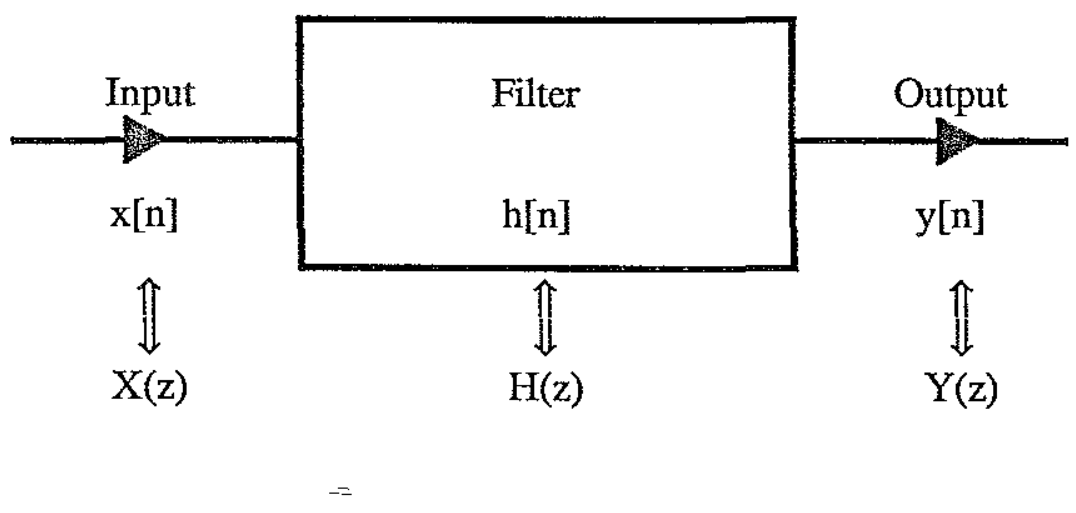

Fig B.1 Discrete Filter Model

The $\mathrm{z}$ transform of a discrete series is an analytic function inside the region of convergence. The $z$ transform of an LCCDE leads to a system function $\left(H(z)=\frac{Y(z)}{X(z)}\right.$ )which is rational. The zeroes of the denominator of $\mathrm{H}(\mathrm{z})$ are singularities known as poles. The zeroes of the numerator are known as zeroes of the system. If the poles of the sytem function are plotted on the complex z plane, the following rules may be applied:

- in order for a system to be stable, the region of convergence (ROC) must contain the unit circle 
- $\mathrm{ROC}$ for a right sided sequence is the area outside a circle with a radius of the outermost pole's magnitude

- for a causal and stable system described by LCCDE, the poles must lie within the unit circle

- the discrete Fourier transform (DFT) is equivalent to evaluating the $\mathrm{z}$ transform at equally spaced points on the unit circle.

\section{All Pole Filters}

Using the LCCDE description of a system, the system function is found by taking the $z$ transform of both sides:

$$
\mathrm{H}(\mathrm{z})=\frac{\sum_{\mathrm{s}=0}^{\mathrm{M}} \mathrm{b}_{\mathrm{s}} \mathrm{z}^{-\mathrm{s}}}{1+\sum_{\mathrm{k}=1}^{\mathrm{p}} \mathrm{a}_{\mathrm{k}} \mathrm{z}^{-\mathrm{k}}}
$$

This $\mathrm{H}(\mathrm{z})$ is a general pole-zero or autoregressive moving average (ARMA) model If all of the numerator coefficients except $b_{0}$ are zero, the system function is:

$$
H\left(f_{2}\right)=\frac{b_{0}}{1+\sum_{k=1}^{\mathrm{P}} a_{k} z^{-k}}
$$

This defines an all pole (also known as an AR or IIR) filter.

Equivalently,

$$
H(z)=\frac{b_{0}}{\prod_{k=1}^{p}\left(1-z_{k} z^{-k}\right)}
$$

This may be represented by a partial fraction expansion as:

$$
\mathrm{H}(\mathrm{z})=\sum_{\mathrm{k}=1}^{\mathrm{p}} \frac{\mathrm{g}_{\mathrm{k}}}{\left(1-\mathrm{z}_{\mathrm{k}} \mathrm{z}^{-\mathrm{k}}\right)}
$$




\section{Appendix C - Prawns Program Listing}

The listing below is the PRAWNS algortihm used in this thesis. Many of the routines are based on those found in Chapter 11 of Marple[31]. Although many of the variable names and comments are the same as Marple's, the PRAWNS program differs markedly. The libraries used include IMSL[57] and PORT[70].

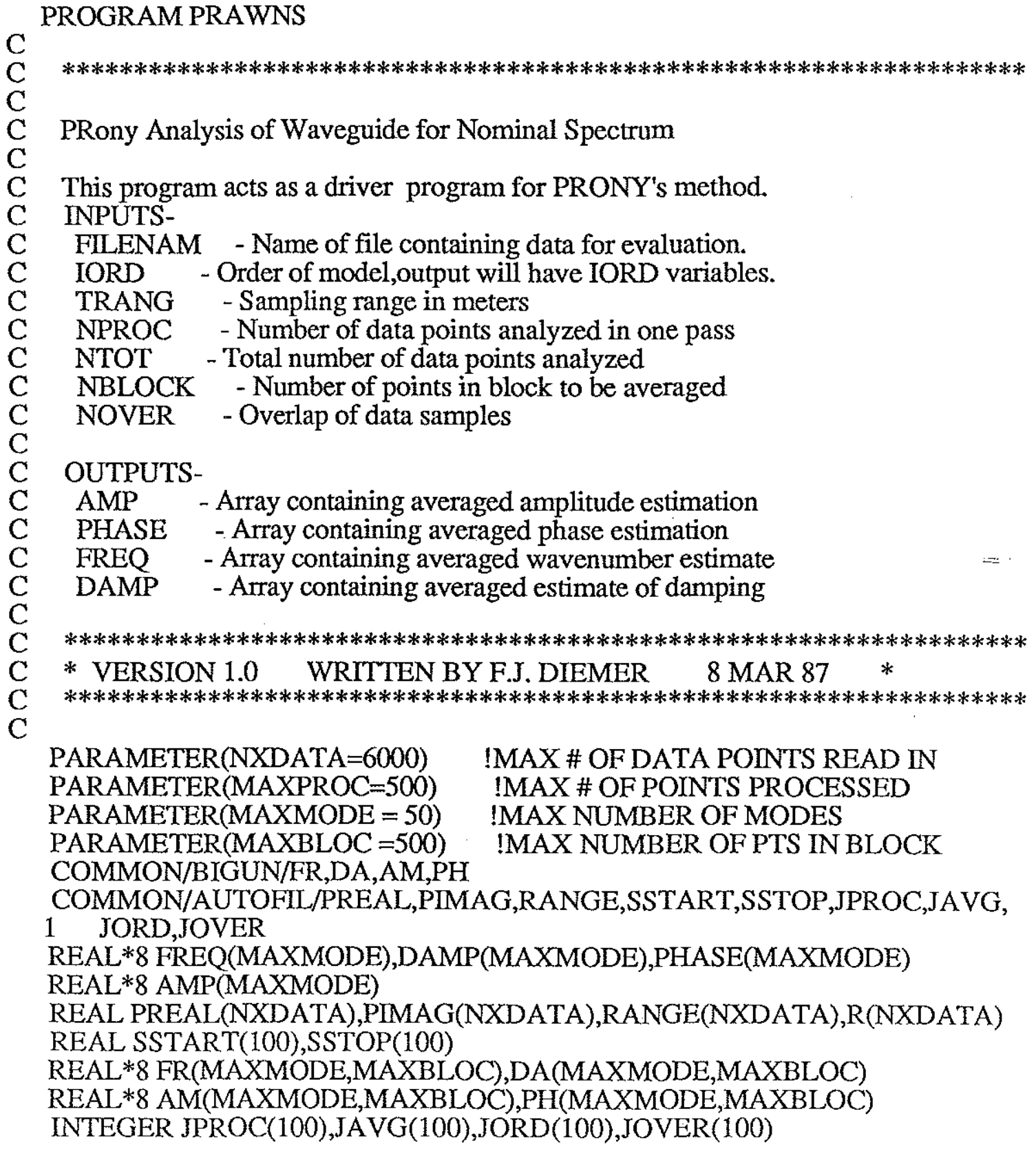




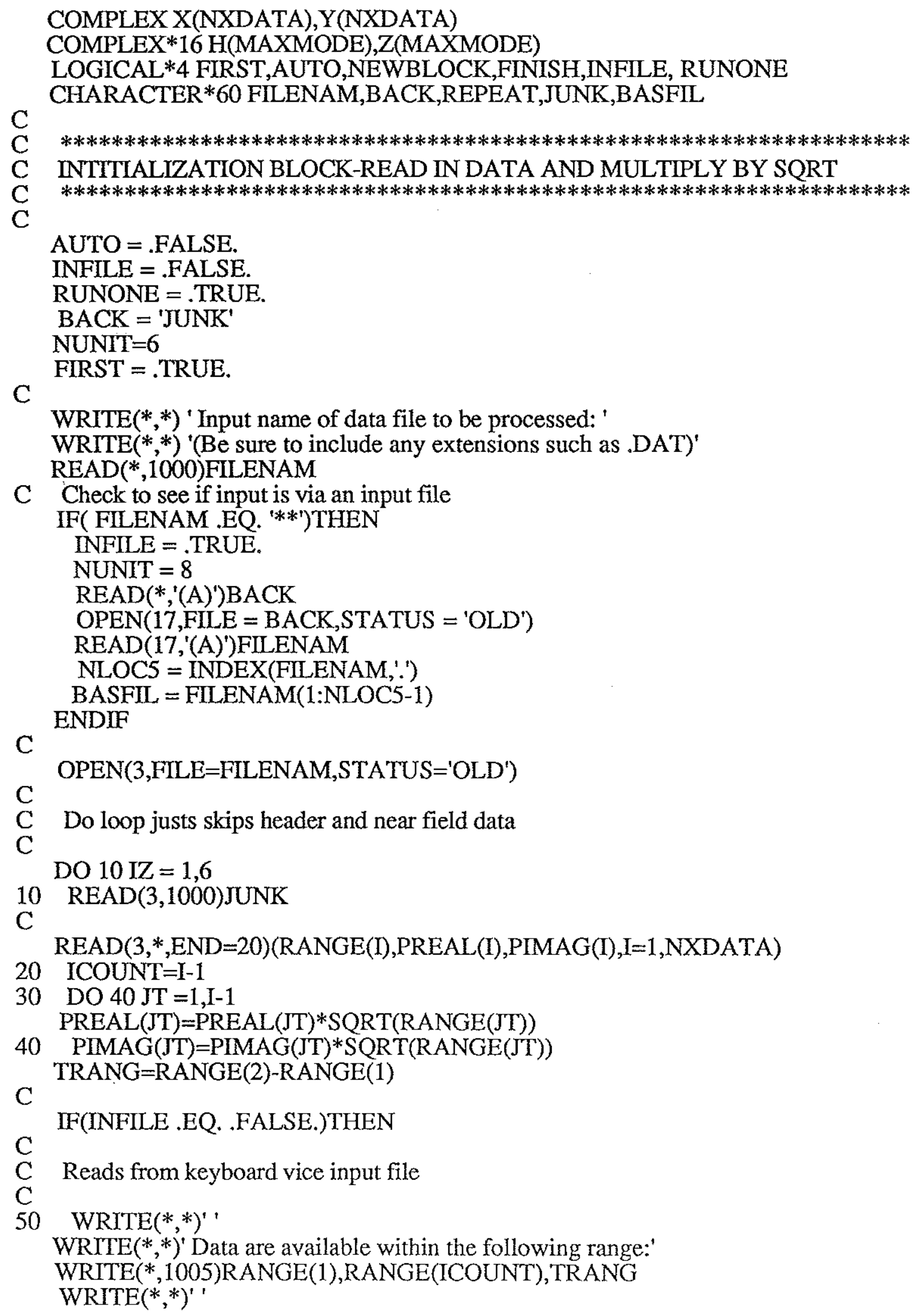


$\mathrm{C}$

WRITE $(*, *)^{\prime}$ Input INTEGER value of START range(in meters):'

CALL READIN(FIRST,MSTART)

RSTART=FLOAT(MSTART)

WRITE $(*, *)^{\prime}$ '

WRITE $(*, *)^{\prime}$ Input INTEGER value of STOP range(in meters):'

CALL READIN(FIRST,MSTOP)

$\mathrm{C}$

RSTOP=FLOAT(MSTOP)

C Determine correct starting and stopping ranges and no of points

C

$\mathrm{RS}=((\mathrm{RSTART}-\mathrm{RANGE}(1)) / \mathrm{TRANG})+1$

IBEGIN=IFIX(RS)

IF(RS-FLOAT(IBEGIN) .NE. 0.0) IBEGIN=IBEGIN+1

ISTOP=IFIX((RSTOP-RANGE(1))/TRANG)+1

NTOT $=($ ISTOP-IBEGIN) +1

$\mathrm{IY}=1$

C

DO $60 \mathrm{JT}=$ IBEGIN,ISTOP

$\mathrm{R}(\mathrm{IY})=\mathrm{RANGE}(\mathrm{JT})$

$60 \quad I Y=I Y+1$

Y(IY)=CMPLX(PREAL(JT),PIMAG(JT))

$\mathrm{C}$

ICOUNT $=$ NTOT

C

WRITE $(*, *)^{\prime}$ '

70 WRTTE $(*, 1010)$ NTOT

C

C

$\mathrm{C}$

C

C

MANUAL AND INITIAL PARAMETER SET BLOCK

************************************************************************

WRITE $(*, *)^{\prime}$ Enter file name for output file or '

WRITE $(*, *)^{\prime}$ hit RETURN to output to screen.'

$\operatorname{READ}\left(*,{ }^{\prime}(\mathrm{A})^{\prime}\right) \mathrm{JUNK}$

IF(JUNK .EQ. ' ') THEN

NUNIT $=6$

ELSE

NUNIT $=8$

OPEN(NUNIT, FILE $=$ JUNK, STATUS $=$ 'NEW')

$\mathrm{C}$ ENDIF

WRITE $(*, *)$ 'Input number of points in each processing block:'

NTRIAL $=$ MIN(MAXPROC,NTOT)

WRITE $(*, 1040)$ NTRIAL

$\mathrm{C}$

CALL READIN(FIRST,NPROC)

C If user requests more than MAXPROC points or more than NTOT

C default value of $\mathrm{NPROC}=20$ is assigned.

$\mathrm{C}$

IF(NPROC .GT. AMINO(MAXPROC,NTOT))NPROC=AMINO(20,NTOT)

IF(ICOUNT/2 .EQ. 0) NPROC = NTEMP 
C

WRITE $(*, *)^{\prime}$ Input number of points overlap between blocks:'

WRITE $(*, 1050)$ NPROC-1

JTEMP=NOVER

CALL READIN(FIRST,NOVER)

IF(NOVER .GT. (NPROC-1)) NOVER $=$ NPROC $/ 2$

IF((JTEMP .EQ. 0) .AND. (NOVER .EQ. 0) .AND. (.NOT. FIRST))

1 NOVER $=0$

IF((JTEMP .NE. 0) .AND. (NOVER .EQ. 0) .AND. (.NOT. FIRST))

1 NOVER = JTEMP

$\mathrm{C}$

$\mathrm{IMAX}=\mathrm{MINO}(50, \mathrm{NPROC} / 2)$

WRITE $(*, *)^{\prime}$ What order system do you want to model: '

WRITE $(*, 1040)$ MMAX

CALL READIN(FIRST,IORD)

IF(IORD .LE. NPROC/2) GOTO 80

WRITE $(*, *)^{\prime}$ Desired order is too high for processing block'

C GO TO 70

80 WRITE(*,*)' '

WRITE(*,*)' Input number of points in averaging block:'

WRITE $(*, 1070)$ NPROC,NTOT

CALL READIN(FIRST,NBLOCK)

IF(NBLOCK .LT. NPROC) NBLOCK $=$ NPROC $\quad$ !Min size of block=NPROC IF(NBLOCK .GT. ntot)NBLOCK=NTOT

C GOTO 110

C

ELSE ! Alternate to sequence started near label 40

C Reads input selections from the input file

$\mathrm{C}$

$\operatorname{READ}(17, *)$ NENTRY

DO 610 JS $=1$,NENTRY

610 READ(17,*)NUMZ,SSTART(JS),SSTOP(JS),JPROC(JS),JOVER(JS),

1 JORD(JS),JAVG(JS)

NAUTO $=1$

CALL

INAUTO(R,Y,NPROC,NOVER,NBLOCK,IORD,BASFIL,NAUTO,TRANG,NTOT)

C

ENDIF

C Initial settings for XSET

C

110 IBLOCK $=$ NBLOCK

NEWBLOCK $=$.TRUE.

IEND $=0$

120 ISTART $=1$

$M=1$

TRES $=0$.

C

$\mathrm{C} \quad * * * * * * * * * * * * * * * * * * * * * * * * * * * * * * * * * * * * * * * * * * * * * * * * * * * * * * * * * * * * * * * * * * *$

C CALCULATION AND LOOP BLOCK

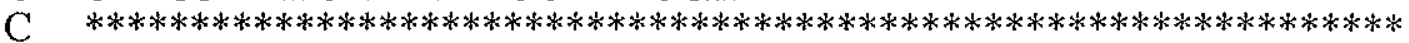


C READ IN CURRENT BLOCK OF DATA

$\mathrm{C}$

130 CALL

XSET(Y,ISTART,IEND,IBLOCK,NTOT,NPROC,NBLOCK,NOVER,NEWBLOCK, 1 FINISH,X)

IF(NEWBLOCK) THEN

ILAST =IEND

IFIRST $=$ IEND $+1-$ NBLOCK

C ENDIF

140 CALL PRONY(NPROC,IORD,X,H,Z,RES,ISTAT)

IF(ISTAT .EQ. 0) GOTO 150

WRITE $(*, *)$ 'PROGRAM HALTED - ERROR NO. ',ISTAT

C GOTO 200

$\mathrm{C} \quad * * * * * * * * * * * * * * * * * * * * * * * * * * * * * * * * * * * * * * * * * * * * * * * * * * * * * * * * * * * * * *$

C The call to EXPARAMS will transform $H$ and $Z$ arrays to final

C output form (Z-->DAMP,FREQ and H--> AMP,PHASE)

$\mathrm{C} \quad * * * * * * * * * * * * * * * * * * * * * * * * * * * * * * * * * * * * * * * * * * * * * * * * * * * * * * * * * * * * * *$

C

150 CALL EXPARAMS(IORD,TRANG,H,Z,AMP,DAMP,FREQ,PHASE)

C

$\mathrm{C}$

C

C

CALL SORT(FREQ,AMP,DAMP,PHASE,IORD,0)

DO $160 \mathrm{NA}=1, \mathrm{IORD}$

$\mathrm{AM}(\mathrm{NA}, \mathrm{M})=\mathrm{AMP}(\mathrm{NA})$

$\mathrm{DA}(\mathrm{NA}, \mathrm{M})=\mathrm{DAMP}(\mathrm{NA})$

FR(NA,M) $=$ FREQ(NA)

$160 \mathrm{PH}(\mathrm{NA}, \mathrm{M})=\mathrm{PHASE}(\mathrm{NA})$

C

TRES $=$ TRES +RES

$\mathrm{M}=\mathrm{M}+1$

IF(.NOT. NEWBLOCK) GOTO 130

$\mathrm{M}=\mathrm{M}-1$

TRES $=$ TRES/M

CALL WHEAD(NUNIT,FILENAM,NTOT,NBLOCK,NPROC,IORD,NOVER,TRANG,

1 R(IFIRST),R(ILAST),AUTO,BACK,IMIN,

1 IMAX,TRES)

CALL THREAD(FREQ,AMP,DAMP,PHASE,M,IORD)

CALL SORT(FREQ,AMP,DAMP,PHASE,IORD, 1)

$\mathrm{C}$

CALL WDATA(NUNIT,JVARY,FREQ,AMP,DAMP,PHASE,IORD,AUTO)

\section{$\mathrm{M}=1$}

FIRST $=$.FALSE.

IF(NOT. FINISH)GOTO 130 !CHECK TO SEE IF AT END OF RANGE

WRITE(NUNIT,1060)

WRITE(NUNIT, 1060)

IF(INFILE .EQ. .TRUE. .AND. NAUTO .NE. NENTRY)THEN

$\mathrm{NAUTO}=\mathrm{NAUTO}+1$ 
CALL

INAUTO(R,Y,NPROC,NOVER,NBLOCK,IORD,BASFIL,NAUTO,TRANG,NTOT) GOTO 110

$\mathrm{C}$

ELSE IF(INFILE .EQ. .FALSE.)THEN

WRITE $(*, *)$ 'Do you want a repeat run of same file with change in'

WRITE $(*, *)$ 'order or method (Y or N)?'

READ $(*, 1000)$ REPEAT

$\mathrm{C}$

IF(REPEAT .EQ. 'Y' .OR. REPEAT .EQ. 'y') GOTO 50

C

ENDIF

200 WRITE(*,*)'Done'

C

1000 FORMAT(A)

1005 FORMAT(1X,'Closest point: ',f9.2,' m.',3x,'Farthest point: ',F9.2,

1 ' $\mathrm{m}$ '/1x,'Sampling interval: ',f7.4,' m.')

1010 FORMAT(1X,' There are ',i5,' samples in the range interval')

1040 FORMAT(1X,' (MAX value $=$ ',i5,')')

1050 FORMAT $\left(1 X,{ }^{\prime}(\right.$ Min overlap $=0$, Max overlap $=$ ',i3,')')

1060 FORMAT 1 X

$1 * * * * * * * * * * * * * * * * * * * * * * * * * * * * * * * * * * * * * * * * * * * * * * * * * * * * * * * * * * * * * * *)$

1070 FORMAT(3X,'(For no averaging between blocks, enter ',i5/t4,

1 'For averaging over complete range, enter ',i5/t4,

1 'Intermediate values will set up blocks of your entry and'/3x,

1 'average within the block.)')

1080 FORMAT(1X,'Completed analysis with ',a6,' = ',i5)

C

END

$\mathrm{C}$

$\mathrm{C}$

$+++++++++++++++++++++++++++++++++++++++++++++++++++++++++++++t$

C

$+++++++++++++++++++++++++++++++++++++++++++++++++++++++++++++t$

SUBROUTINE INAUTO(R,Y,JA,JB,JC,JD,FILENAM,NAUTO,TRANG,NTOT)

$\mathrm{C}$

$\mathrm{C} \quad * * * * * * * * * * * * * * * * * * * * * * * * * * * * * * * * * * * * * * * * * * * * * * * * * * * * * * * * * * * * * * * * * * *$

C INAUTO assigns the next set of inputs from the input file to

$\mathrm{C}$ the variable names used by PRONY. In addition, the range block

$\mathrm{C}$ interest is read into the $\mathrm{R}$ and $\mathrm{Y}$ arrays and an output file is

$\mathrm{C}$ opened under NUNIT $=8$.

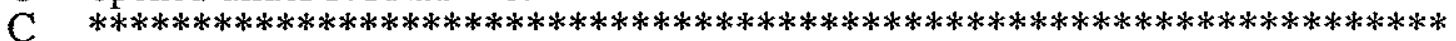

C

PARAMETER(NXDATA $=6000)$

COMMON/AUTOFIL/PREAL,PIMAG,RANGE,SSTART,SSTOP,JPROC,JAVG, 1 JORD,JOVER

REAL PREAL(NXDATA),PIMAG(NXDATA),RANGE(NXDATA),SSTART(100)

REAL SSTOP(100),R(NXDATA)

COMPLEX Y(NXDATA)

INTEGER JAVG(100),JORD(100),JPROC(100),JOVER(100)

$\mathrm{C}$

CHARACTER *80 FILENAM,SCRATCHA,SCRATCHB 


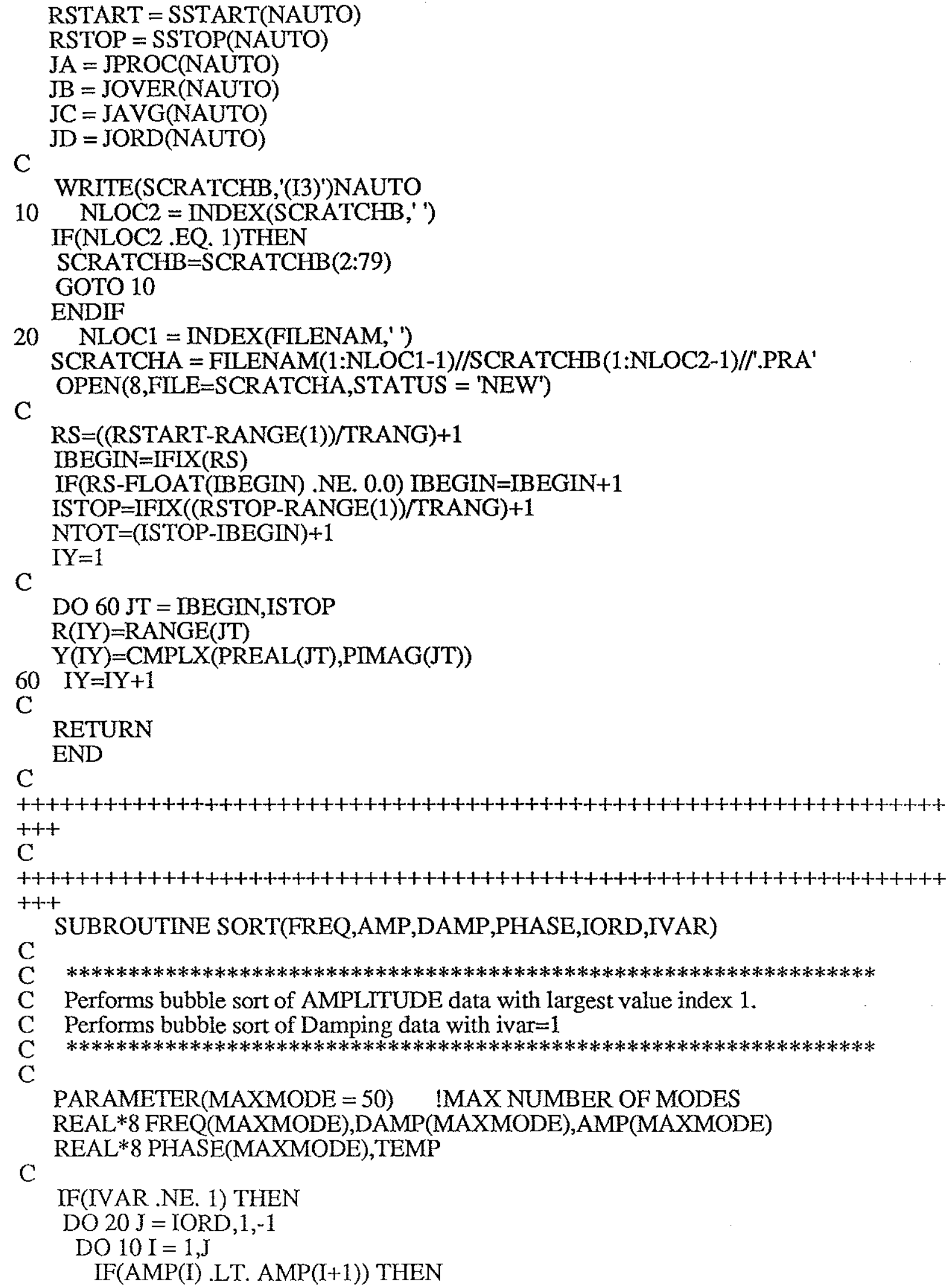




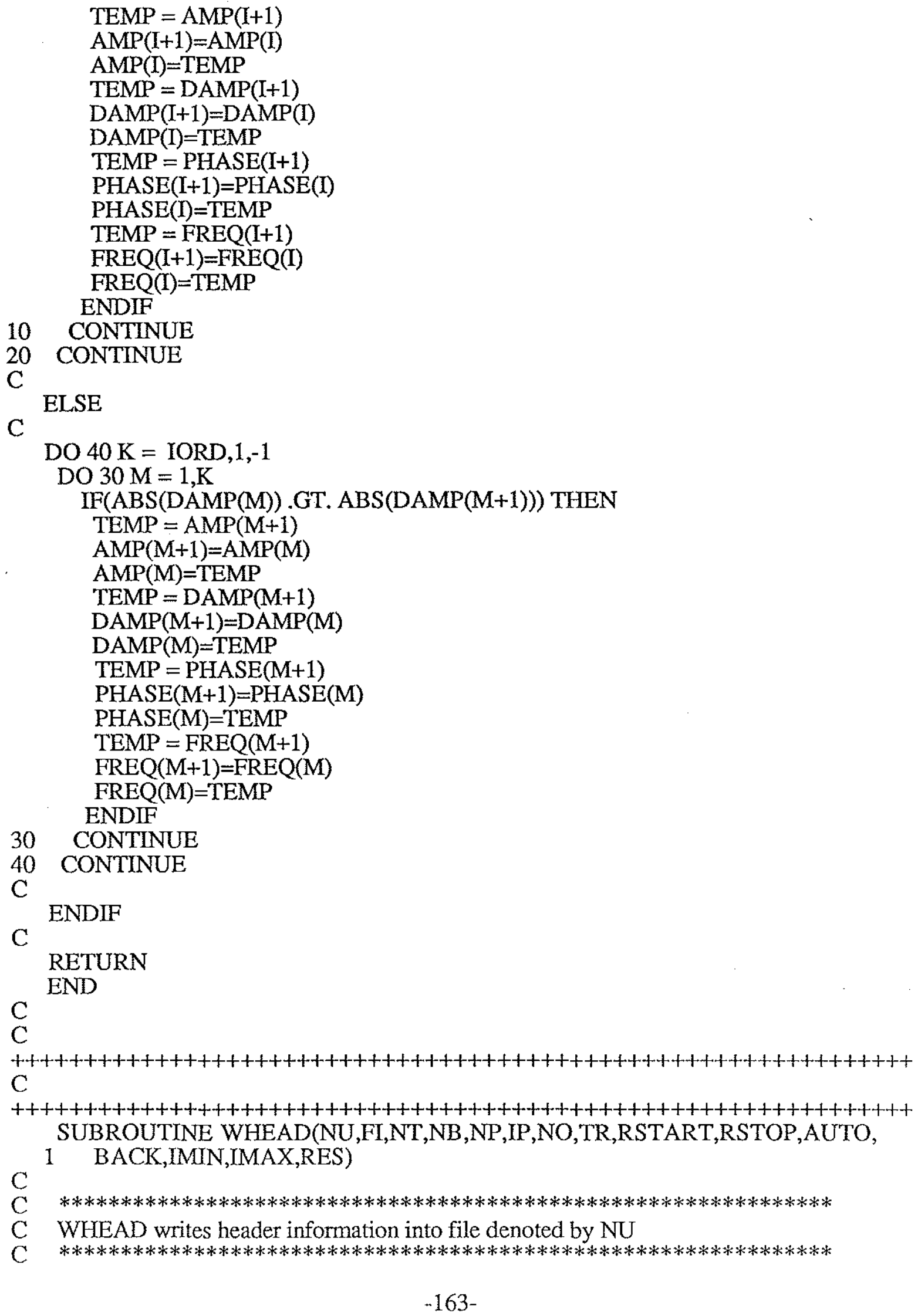


C

$\mathrm{C}$

CHARACTER*60 FI,BACK

LOGICAL AUTO

WRITE(NU,1050)

WRITE(NU,1000)FI,NT,NB,NP,IP,NO,TR

WRITE(NU,1010)RSTART,RSTOP

C

WRITE(NU,1040)RES

IF(AUTO)THEN

WRITE(NU,1020)BACK,IMIN,IMAX,BACK

ELSE

WRITE(NU,1030)'INDEX','WAVENUMBER','DAMPING','AMPLITUDE', 1 'PHASE(RAD)'

$\mathrm{C}$ ENDIF

1000 FORMAT(1X,'Prony Analysis of: ',a60//t10,'Total No. of points: ',

1 I5,13x,'Avg. block:',i3,' pts.'/t10,'Processing block: ',

1 i3,17x,'Model Order: ',i2/t10,'Overlap: ',i3,' pts.',21x,

1 'Samp. Range: ',f7.4,' m.')

1010 FORMAT(1X,T10,'Starting Range: ',f10.4,' m.',8x,'Final Range:',

1 f10.4,' m.')

1020 FORMAT(1X,A6,' varied from ', I3,' to ',I3/T2,A6,5x,

1 'Wavenumber',5x,'Amplitude')

1030 FORMAT(1X,A5,3X,A10,6X,A7,7X,A9,6X,A10)

1040 FORMAT( $9 X$, ,'Residue of model for this range interval: ',

1 F8.5//)

1050 FORMAT(1X, 1

' +++++++++++++++++++++++++++++++++++++++++++++++++++++++++++1,

$\mathrm{C}$

$1+++1)$

RETURN

END

$\mathrm{C}$

C

$+++++++++++++++++++++++++++++++++++++++++++++++++++++++++++++++$

$\mathrm{C}$

$+++++++++++++++++++++++++++++++++++++++++++++++++++++++++++++++$ C

SUBROUTINE WDATA(NUNIT,JV,FREQ,AMP,DAMP,PHASE,IORD,AUTO)

$\mathrm{C} \quad * * * * * * * * * * * * * * * * * * * * * * * * * * * * * * * * * * * * * * * * * * * * * * * * * * * * * * * * * * * * * * * * *$

C WDATA writes appropriate data to file specified by NUNIT

$\mathrm{C} * * * * * * * * * * * * * * * * * * * * * * * * * * * * * * * * * * * * * * * * * * * * * * * * * * * * * * * * * * * * * * * * * *$

$\mathrm{C}$

PARAMETER(MAXMODE $=50) \quad$ !MAX NUMBER OF MODES

REAL*8 FREQ(MAXMODE),DAMP(MAXMODE),AMP(MAXMODE)

REAL*8 PHASE(MAXMODE)

C

LOGICAL AUTO

IF(AUTO) THEN

DO $10 \mathrm{~J}=1$,IORD 


\section{ELSE}

WRITE(NUNIT, 1000)JV,FREQ(J),AMP(J)

DO $20 \mathrm{M}=1, \mathrm{IORD}$

20 WRITE(NUNIT,1020)M,FREQ(M),DAMP(M),AMP(M),PHASE(M)

C ENDIF

1000 FORMAT(1X,I3,5X,2(F10.6,2X))

1020 FORMAT(1X,I2,4(5X,F10.7))

C

\section{RETURN}

C

END

C

$+++++++++++++++++++++++++++++++++++++++++++++++++++++++++++++++$

C

$+++++++++++++++++++++++++++++++++++++++++++++++++++++++++++++++$ SUBROUTINE

XSET(Y,ISTART,IEND,IBLOCK,IFINAL,NPROC,NBLOCK,NOVER,

$\mathrm{C}$

1 NEWBLOCK,FINISH,X)

C

C Xset is a routine to output the correct X array for PRONY

$\mathrm{C}$ analysis. The routine uses values from $\mathrm{Y}$ and takes into account

$\mathrm{C}$ the processing block, averaging block and total range covered.

$\mathrm{C}$ Variables beginning with " $\mathrm{N}$ " indicate number of points while

$\mathrm{C}$ variables beginning with " $\mathrm{I}$ " indicate index pointer.

$\mathrm{C} \quad * * * * * * * * * * * * * * * * * * * * * * * * * * * * * * * * * * * * * * * * * * * * * * * * * * * * * * * * * * * * * * * *$

C

PARAMETER $($ NXMAX $=100)$

PARAMETER (NYMAX $=600)$

COMPLEX X(NXMAX),Y(NYMAX)

C

LOGICAL NEWBLOCK,FINISH

C

FINISH $=$.FALSE.

IF(IEND .EQ. IFINAL) THEN

FINISH $=$.TRUE.

NEWBLOCK $=$.TRUE.

RETURN

C

ENDIF

C

IF (.NOT. NEWBLOCK) THEN

IF((IEND + NPROC - NOVER) .LT. IBLOCK) THEN

ISTART $=$ IEND + 1-NOVER

IEND $=$ ISTART + NPROC -1

ELSE

IEND $=$ IBLOCK

ISTART $=$ IEND $+1-$ NPROC

NEWBLOCK $=$.TRUE.

IBLOCK $=$ IBLOCK + NBLOCK

IF(IBLOCK .GT. IFINAL) IBLOCK = IFINAL 


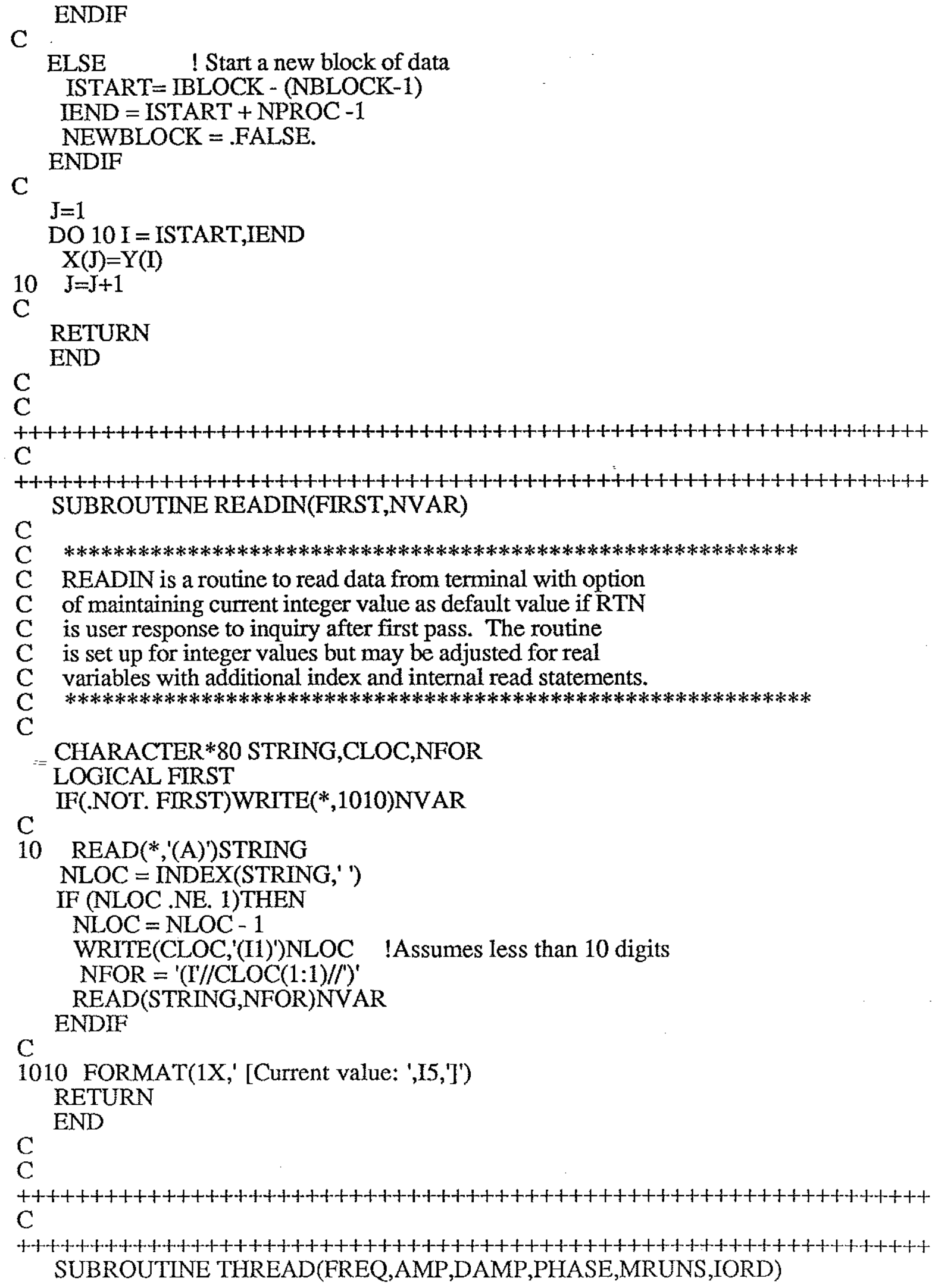




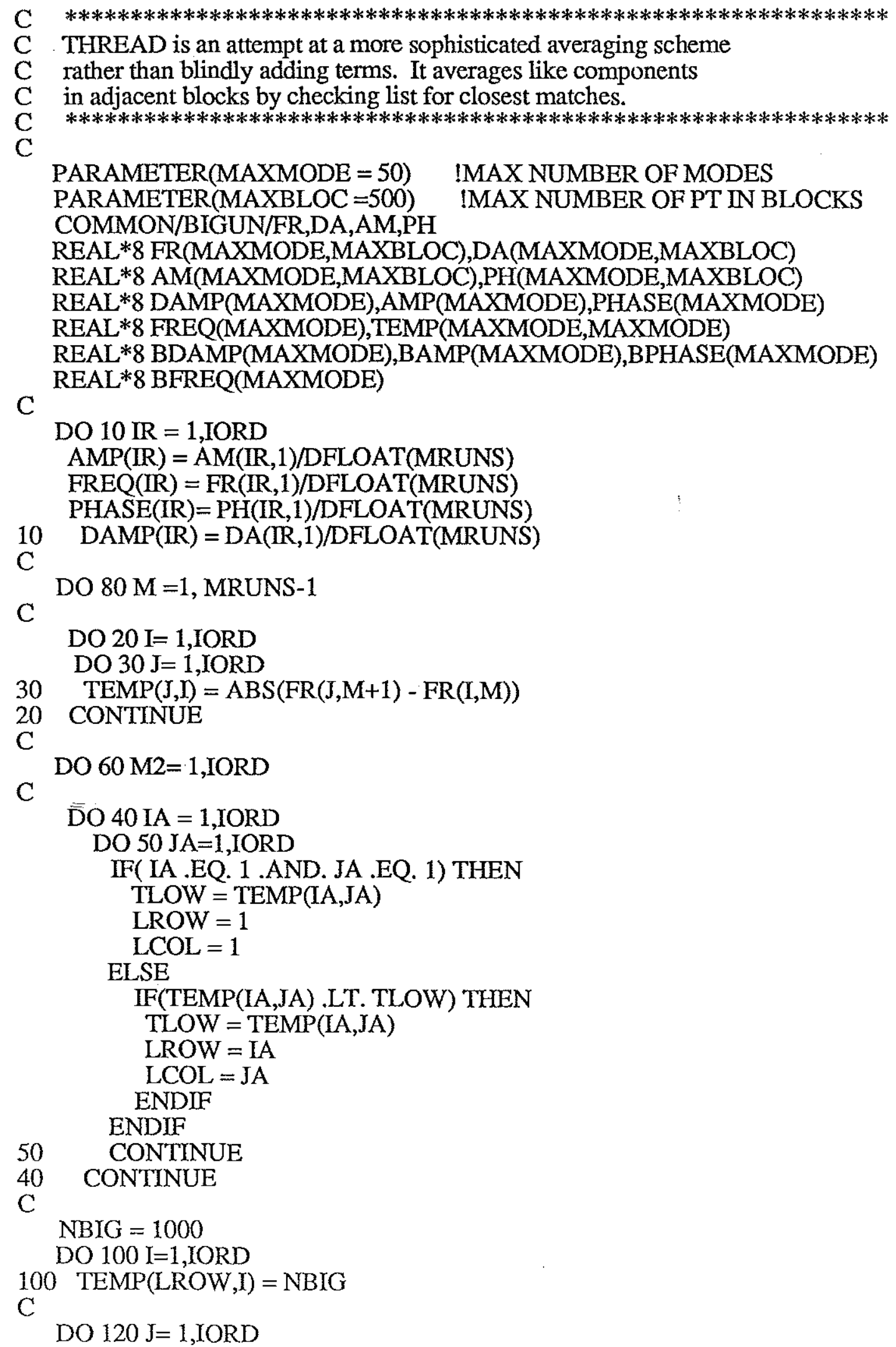


C

$$
\begin{aligned}
& \text { AMP(LCOL) }=\text { AMP(LCOL) }+(\text { AM(LROW,M+1)/DFLOAT(MRUNS })) \\
& \text { BAMP(LROW) =AMP(LCOL) } \\
& \text { FREQ(LCOL) = FREQ(LCOL) }+(\text { FR(LROW,M+1)/DFLOAT(MRUNS })) \\
& \text { BFREQ(LROW) = FREQ(LCOL) } \\
& \text { PHASE(LCOL) = PHASE(LCOL) }+(\text { PH(LROW,M+1)/DFLOAT(MRUNS })) \\
& \text { BPHASE(LROW) = PHASE(LCOL) } \\
& \text { DAMP(LCOL) = DAMP(LCOL) }+(\text { DA(LROW,M+1)/DFLOAT(MRUNS) }) \\
& \text { BDAMP(LROW) = DAMP(LCOL) } \\
& \text { CONTINUE }
\end{aligned}
$$

$\mathrm{C}$

DO $70 \mathrm{M} 3=1$,IORD

$\mathrm{DAMP}(\mathrm{M} 3)=\mathrm{BDAMP}(\mathrm{M} 3)$

FREQ(M3) $=$ BFREQ(M3)

PHASE(M3) $=$ BPHASE(M3)

$70 \quad \mathrm{AMP}(\mathrm{M} 3)=\mathrm{BAMP}(\mathrm{M} 3)$

$\mathrm{C}$

80 CONTINUE

C

\section{RETURN}

END

C

SUBROUTINE PRONY (N,IP,X,H,Z,ERR,ISTAT)

C Solves for the exponential model parameters by the Prony

C method

C Input parameters:

C

C

C N -Number of data samples (integer)

C IP -Order of exponential model (integer)

C X -Array of complex data samples X(1) through $\mathrm{X}(\mathrm{N})$

$\mathrm{C}$

C Output parameters:

C

C H -Array of exponential model complex amplitudes

C Z -Array of exponential model complex exponents

C ISTAT -Integer status indicator at time of exit

C 4 - error exists in routine CHOLESKY

C

C Notes:

C

C External arrays $\mathrm{H}, \mathrm{Z}$ muust be dimensioned .GE. IP and array $\mathrm{X}$

C must be dimensioned .GE. $N$ in calling program. Internal array

C B must be dimensioned .GE. IP(IP+1)/2; arrays A, ROOTR, ROOTI

C must be dimensioned .GE. IP; arrays PR,PI must be dimensioned

C .GE. IP+1. Array $G$ must be dimensioned .GE. IP/2.

$\mathrm{C}$

C Subroutine CHOLESKY required.

$\mathrm{C}$

PARAMETER $($ NMAX $=500)$

PARAMETER (MAXMODE=50)

IMAX NUMBER OF POINTS PROCESSED !MAX NUMBER OF MODES 


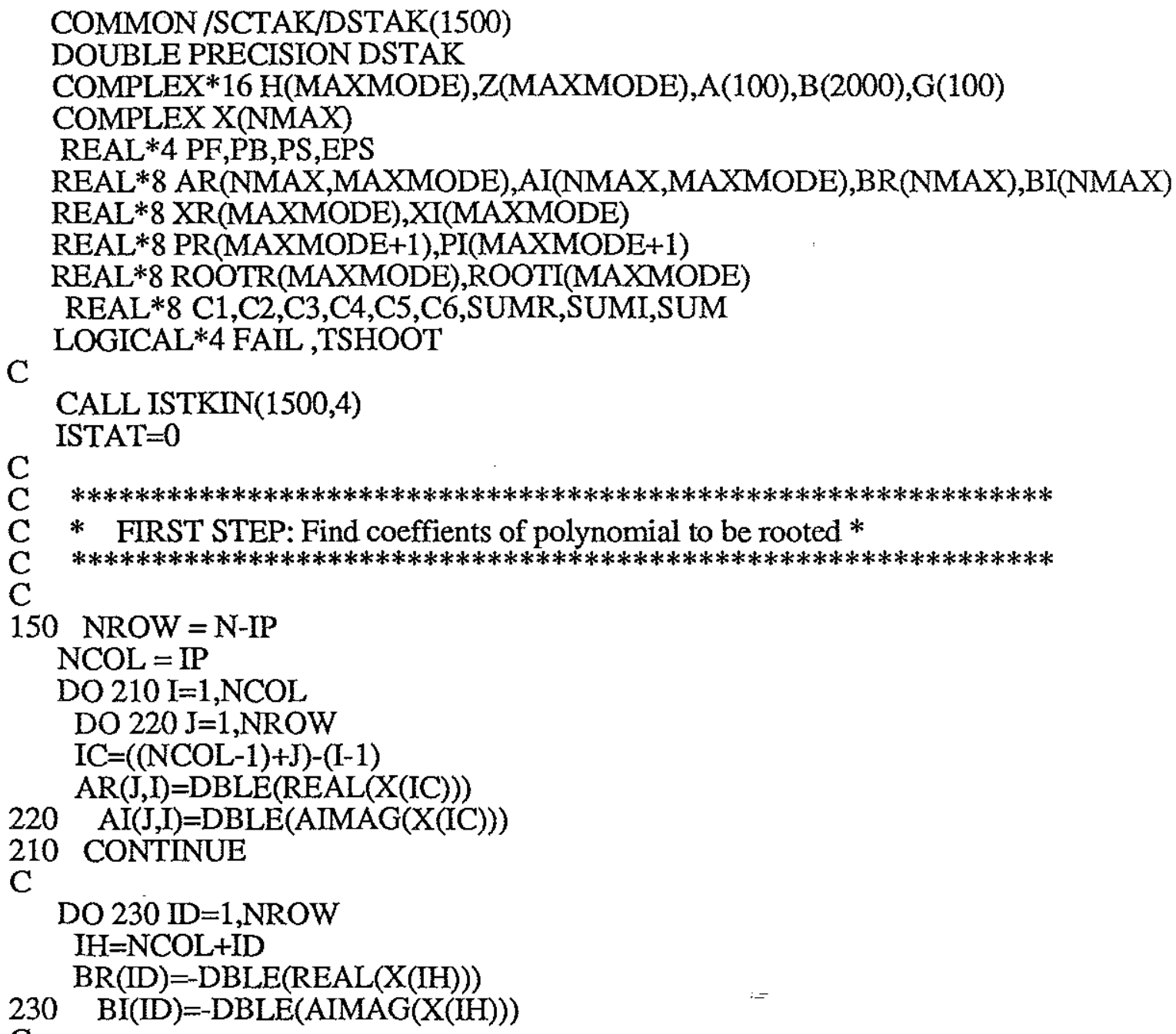

CALL DCLST2(NMAX,MAXMODE,NROW,NCOL,AR,AI,BR,BI,1, $1 \quad \mathrm{XR}, \mathrm{XI})$

$\mathrm{PR}(1)=1 . \mathrm{D} 0$

$\mathrm{PI}(1)=0 . \mathrm{D} 0$

DO $240 \mathrm{IV}=1, \mathrm{NCOL}$ !sets up roots for DCPOLY

$\mathrm{PR}(\mathrm{IV}+1)=\mathrm{XR}(\mathrm{IV})$

$240 \quad \mathrm{PI}(\mathrm{IV}+1)=\mathrm{XI}(\mathrm{IV})$

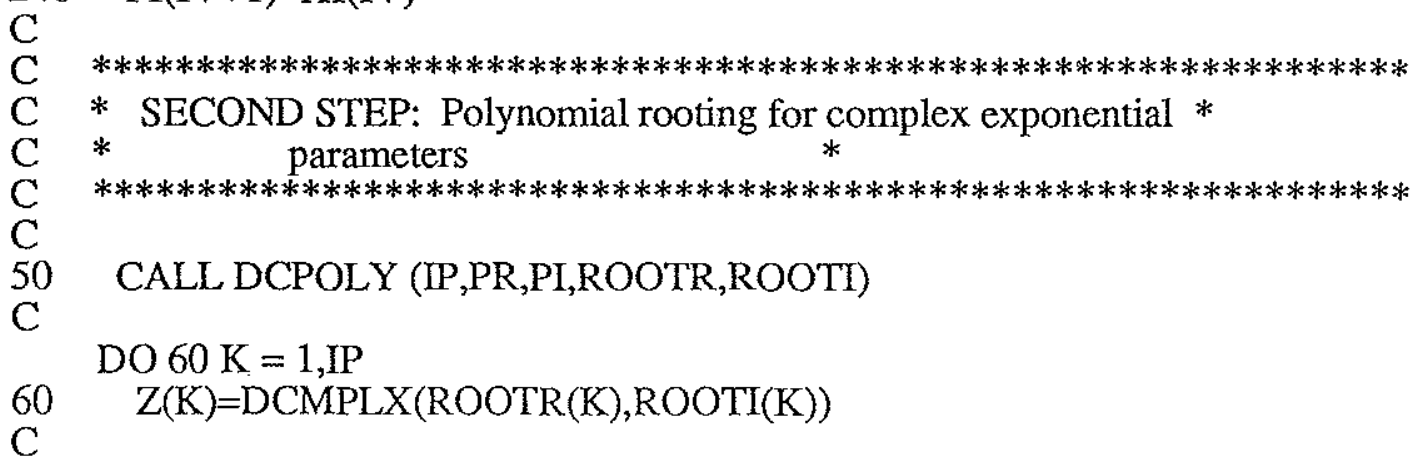




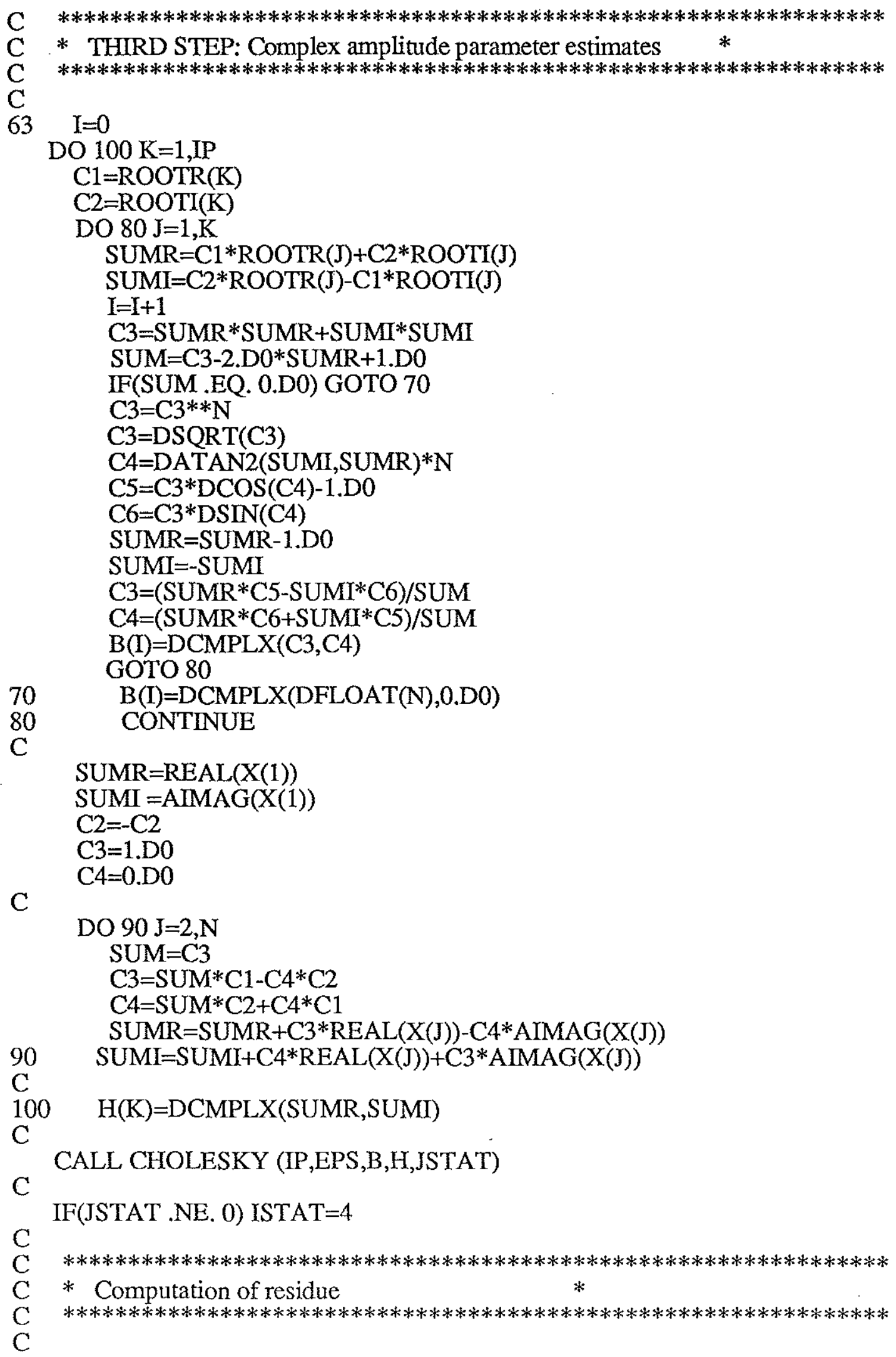


$\mathrm{C}$

CALL RESIDUAL(X,PR,PI,IP,N,ERR)

RETURN

END

SUBROUTINE RESIDUAL(X,PR,PI,IORD,NPROC,ERR)

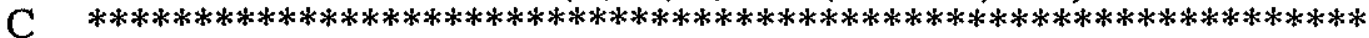

C RESIDUAL is a routine which computes energy of error between

C model and the data. The data is modelled as an all pole filter

C and, if the data is passed through FIR filter with same

C filter coefficients as denominator of IIR model, should yield

C an impulse. An impulse is subtracted from convolution of

$\mathrm{C}$ data and FIR filter and remaining energy is found (as ERR).

$\mathrm{C} \quad * * * * * * * * * * * * * * * * * * * * * * * * * * * * * * * * * * * * * * * * * * * * * * * * * * * * * * * * * * * * * * * *$

REAL*8 PR(1),PI(1)

REAL FILR(4096),FILI(4096),VALR(4096),VALI(4096)

COMPLEX X(1)

LOGICAL SKIP

SKIP $=$. FALSE.

NCONV $=$ IORD + NPROC $\quad$ !Required length of conv/FFT

NFFT $=0$

C Find FFT order-should be radix 2 .GE. NPPROC + (IORD+1) - 1

DO $10 \mathrm{I} 1=1,12$

IF(SKIP) GOTO 10

NTRY $=2 * * I 1$

IF(NTRY .GE. NCONV) THEN

$\mathrm{NFFT}=\mathrm{I1}$

SKIP $=$.TRUE.

ENDIF

10 CONTINUE

C Order of FFT is NFFT. Now zero pad array

DO $20 \mathrm{~J} 1=1$, NTRY

FILR(J1) $=0.0$

FILI $(\mathrm{J} 1)=0.0$

$\operatorname{VALR}(\mathrm{J} 1)=0.0$

$20 \operatorname{VALI}(\mathrm{J} 1)=0.0$

C Fill FFT input arrays with values

DO $30 \mathrm{M} 2=1, \mathrm{IORD}+1$

FILR(M2) $=$ SNGL(PR(M2))

30 FILI(M2) $=$ SNGL(PI(M2))

DO $40 \mathrm{M} 3=1, \mathrm{NPROC}$

VALR(M3) $=$ REAL $(X(M 3))$

40 VALI(M3) = AIMAG(X(M3))

C Call FFT program; convolution is accomplished by FFT of input

$\mathrm{C}$ and filter, multiplying result point by point and IFFT.

CALL FFT842(0,NTRY,FILR,FILI)

CALL FFT842(0,NTRY,VALR,VALI)

$\mathrm{C}$ Point by point multiplication

DO $50 \mathrm{M}=1$, NTRY

TEMPR $=$ FILR(M) $*$ VALR $(M)-$ FILI(M) $*$ VALI(M)

TEMPI $=$ FILR $(M) *$ VALI $(M)+$ FILI(M)*VALR(M)

VALR $(M)=$ TEMPR

$50 \quad$ VALI $(M)=$ TEMPI 
C Now IFFT for convolution result- perfect model yields impulse CALL FFT842(1,NTRY, VALR, VALI)

C Subtract impulse and sum residual for energy $\mathrm{ERR}=0.0$

C SUM FROM 1ST POINT AWAY FROM ORIGIN TO LENGTH OF SEQUENCE DO $60 \mathrm{M} 4=2$,NTRY

$60 \quad \mathrm{ERR}=\mathrm{ERR}+($ VALR(M4) *VALR(M4) + VALI(M4)*VALI(M4)) $\mathrm{ERR}=\mathrm{ERR} /(\mathrm{NTRY}-1)$ RETURN END

C-

C SUBROUTINE: FFT842

C FAST FOURIER TRANSFORM FOR $N=2 * * M$

C COMPLEX INPUT

$\mathrm{C}$

$\mathrm{C}$

C

SUBROUTINE FFT842(IN, N, X, Y)

C THIS PROGRAM REPLACES THE VECTOR $Z=X+I Y$ BY ITS FINITE C DISCRETE, COMPLEX FOURIER TRANSFORM IF IN $=0$. THE INVERSE TRANSFORM C IS CALCULATED FOR IN=1. IT PERFORMS AS MANY BASE C 8 TTERATIONS AS POSSIBLE AND THEN FINISHES WITH A BASE 4 ITERATION C OR A BASE 2 ITERATION IF NEEDED. $\mathrm{C}$

C THE SUBROUTINE IS CALLED AS SUBROUTINE FFT842 (IN,N,X,Y). C THE INTEGER N (A POWER OF 2), THE N REAL LOCATION ARRAY X, AND C THE N REAL LOCATION ARRAY Y MUST BE SUPPLIED TO THE SUBROUTINE. $\mathrm{C}$

DIMENSION X(2), Y(2), L(15)

COMMON/CON2/ PI2, P7 EQQUIVALENCE (L15,L(1)), (L14,L(2)), (L13,L(3)), (L12,L(4)),

* (L11,L(5)), (L10,L(6)), (L9,L(7)), (L8,L(8)), (L7,L(9)),

* (L6,L(10)), (L5,L(11)), (L4,L(12)), (L3,L(13)), (L2,L(14)),

$\mathrm{C}$

* (L1,L(15))

C

C IW IS A MACHINE DEPENDENT WRTTE DEVICE NUMBER

C

C

$\mathrm{IW}=\mathrm{I} 1 \mathrm{MACH}(2)$

$\mathrm{PI} 2=8 . * \mathrm{ATAN}(1$.

$\mathrm{P} 7=1 . / \mathrm{SQRT}(2$.

DO $10 \mathrm{I}=1,15$

$\mathrm{M}=\mathrm{I}$

$\mathrm{NT}=2 * * \mathrm{I}$

IF (N.EQ.NT) GO TO 20

10 CONTINUE

WRITE (TW,9999)

9999 FORMAT (35H N IS NOT A POWER OF TWO FOR FFT842)

STOP

$20 \mathrm{~N} 2 \mathrm{POW}=\mathrm{M}$ 


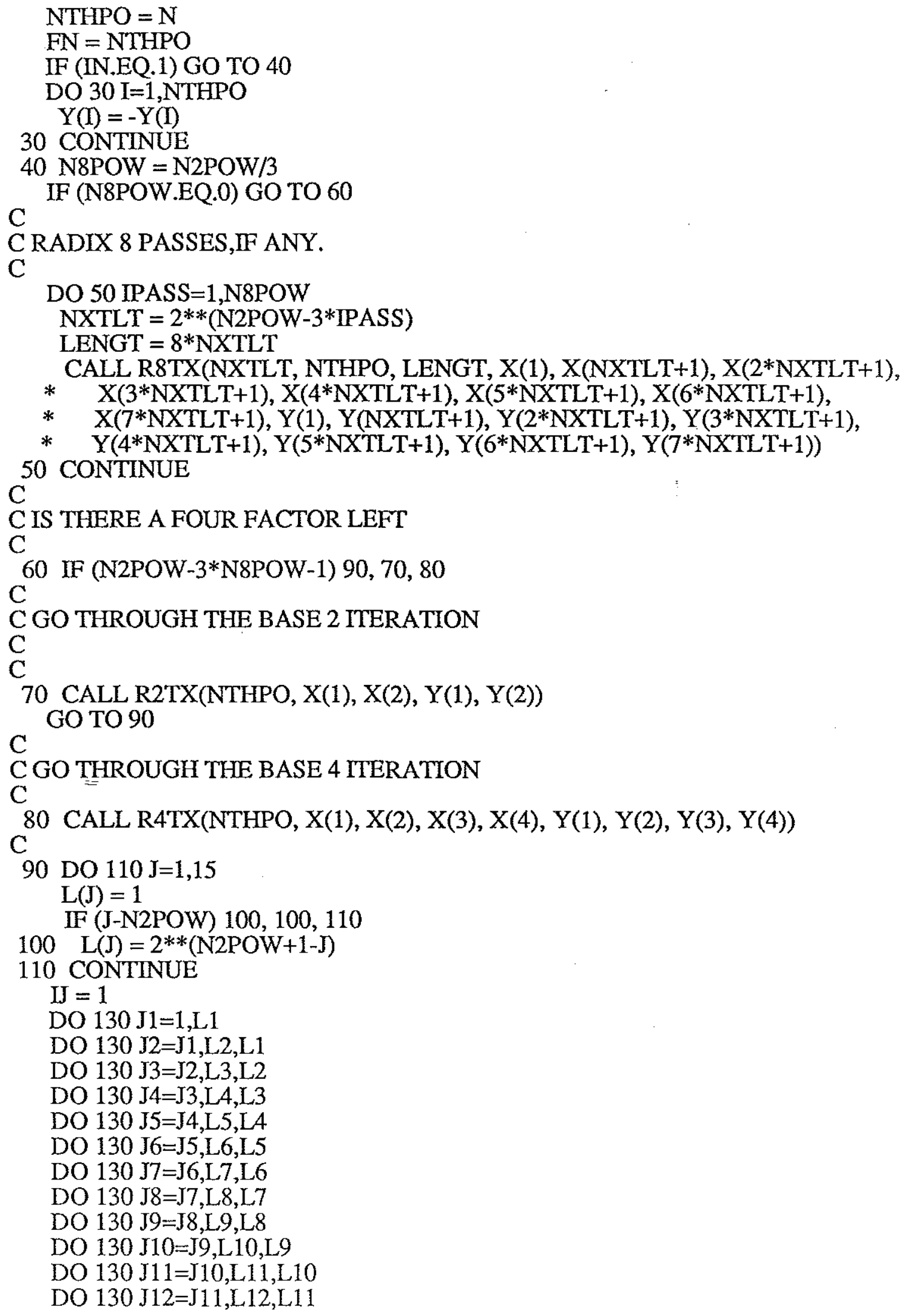




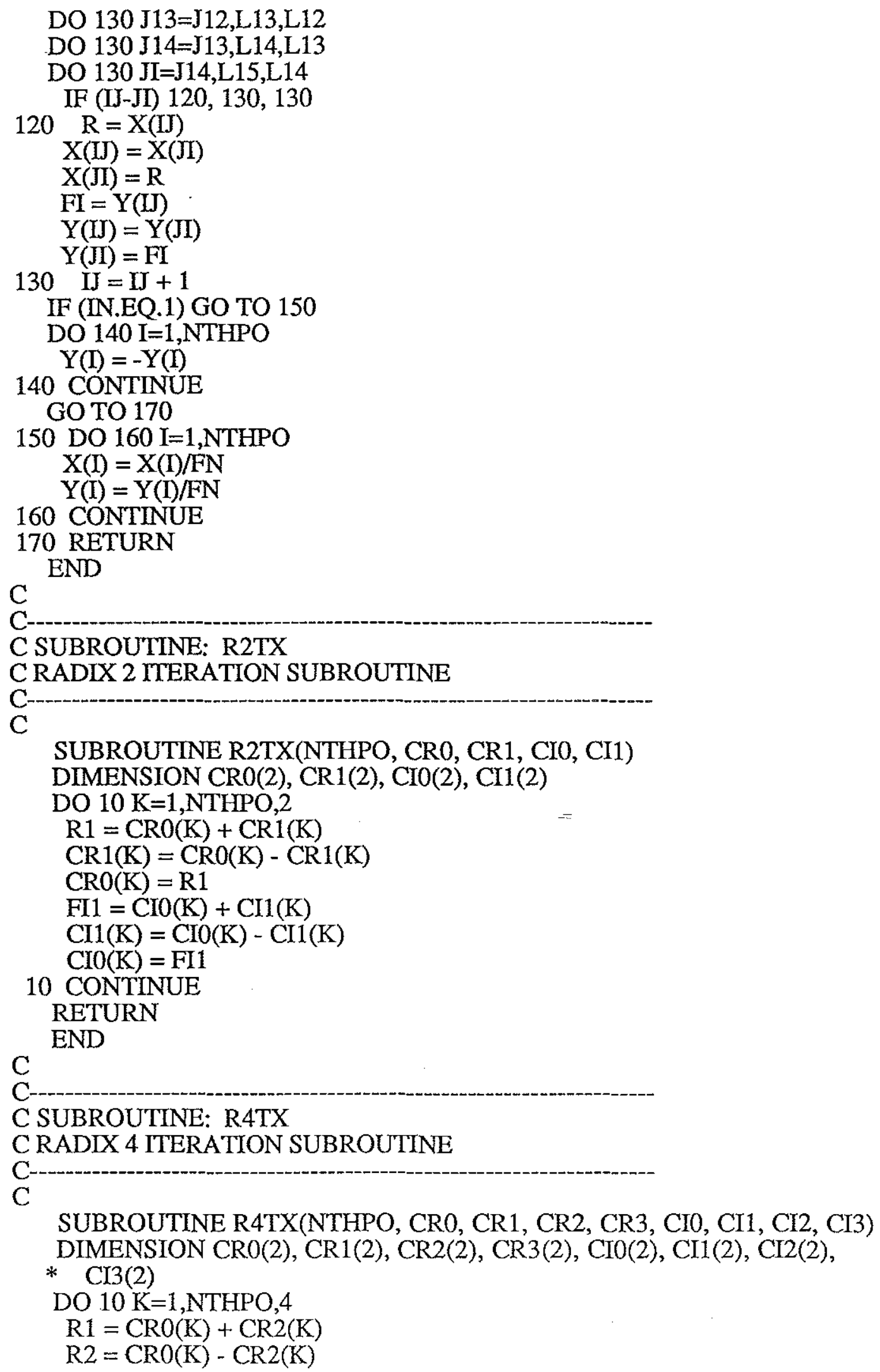




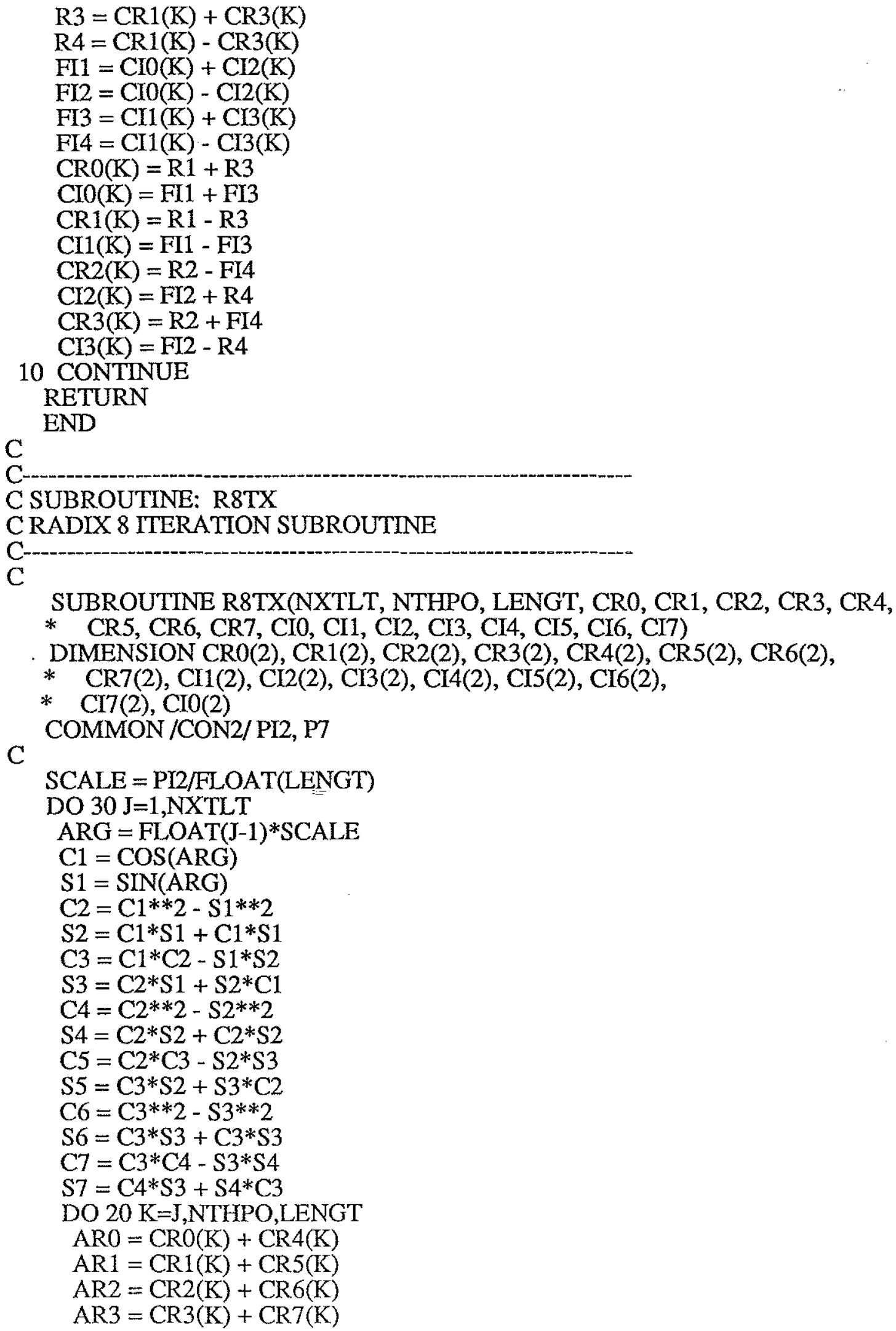




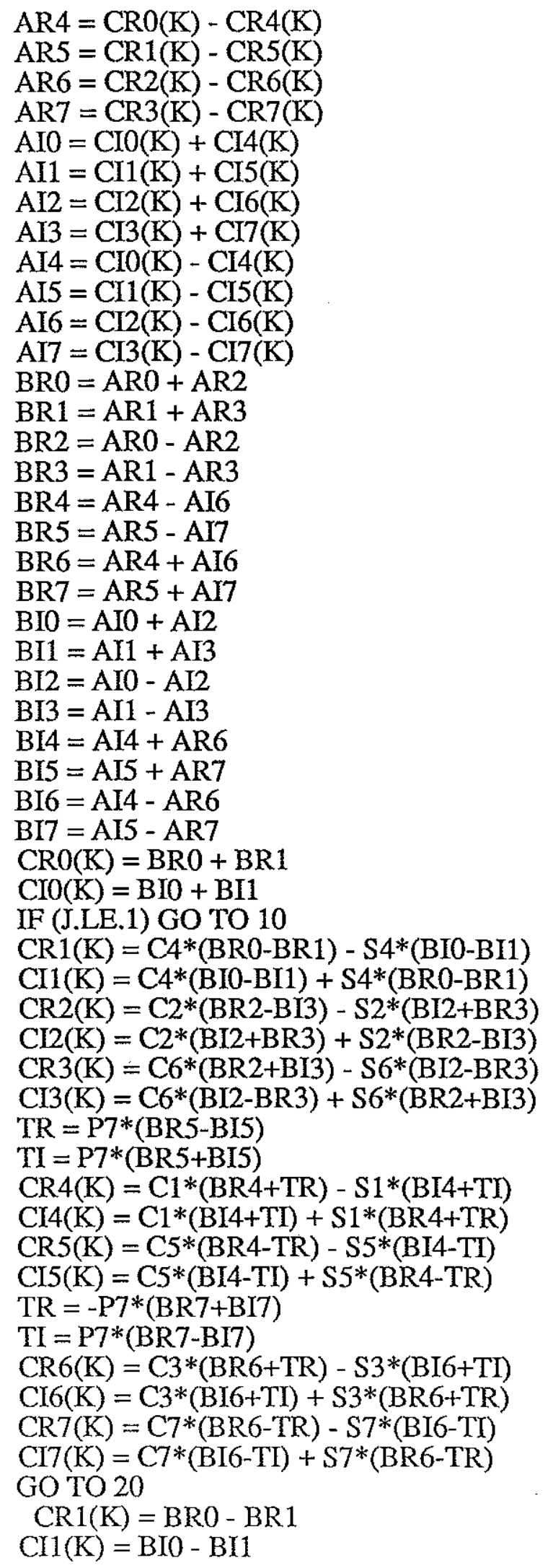




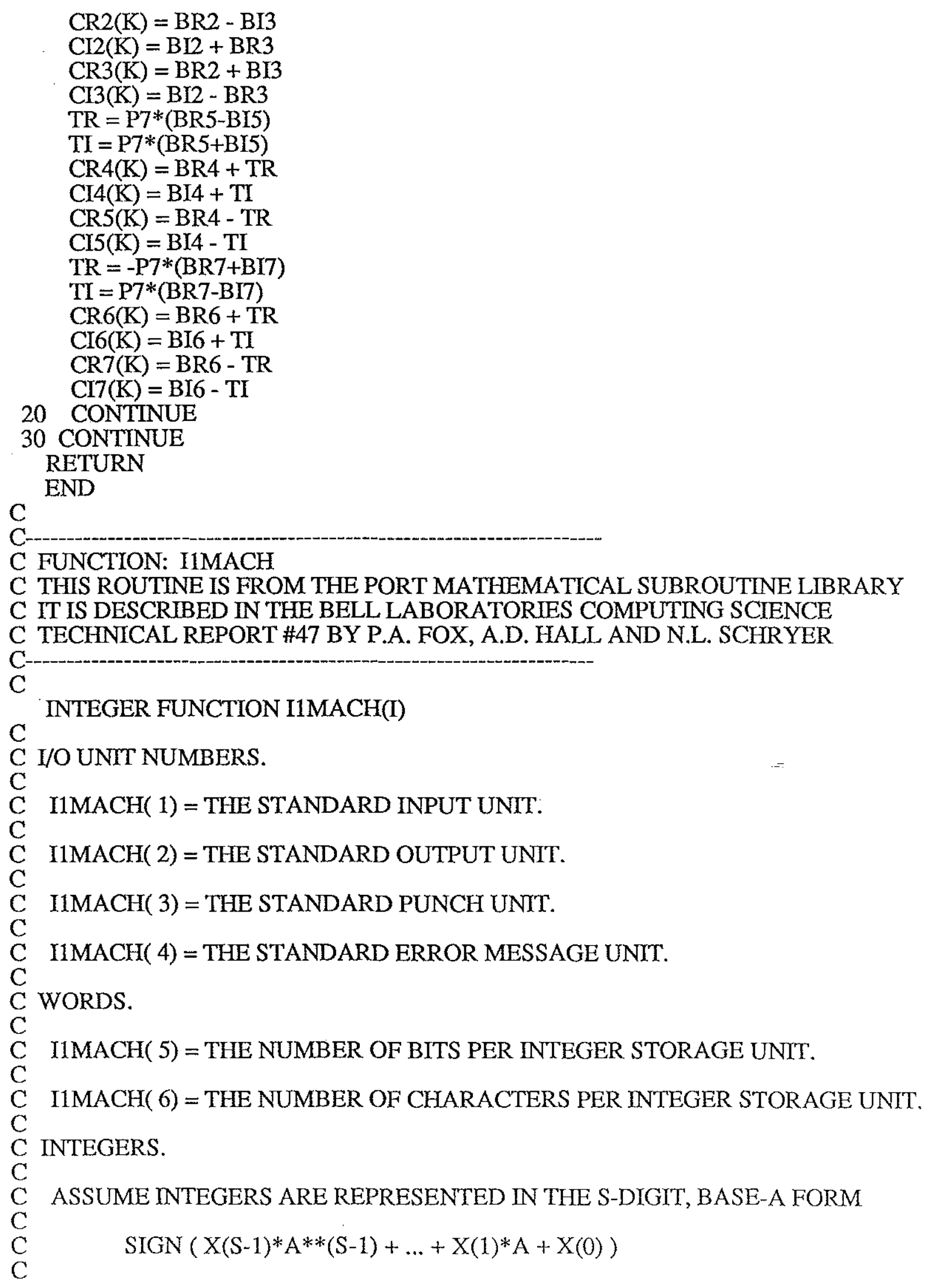




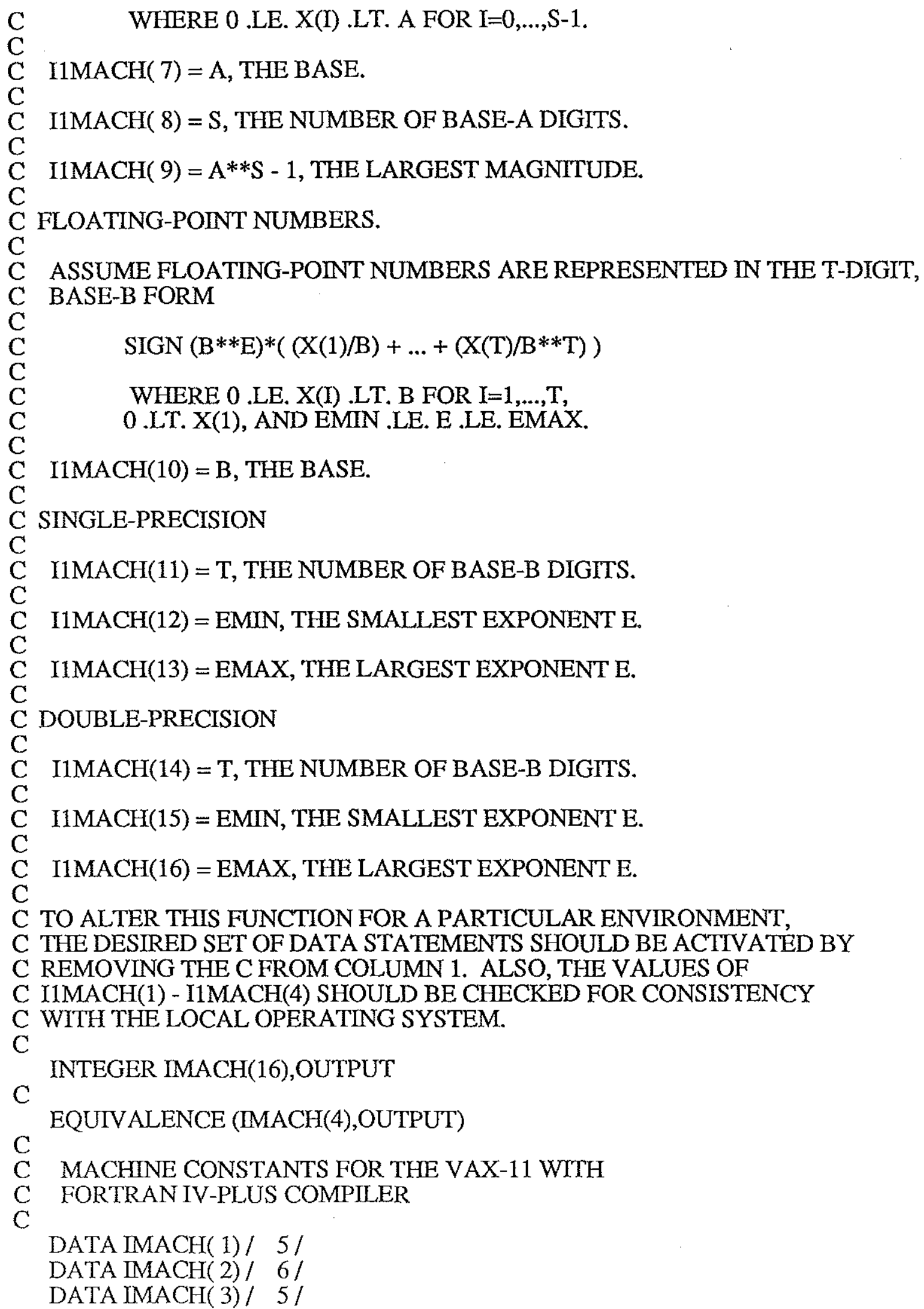




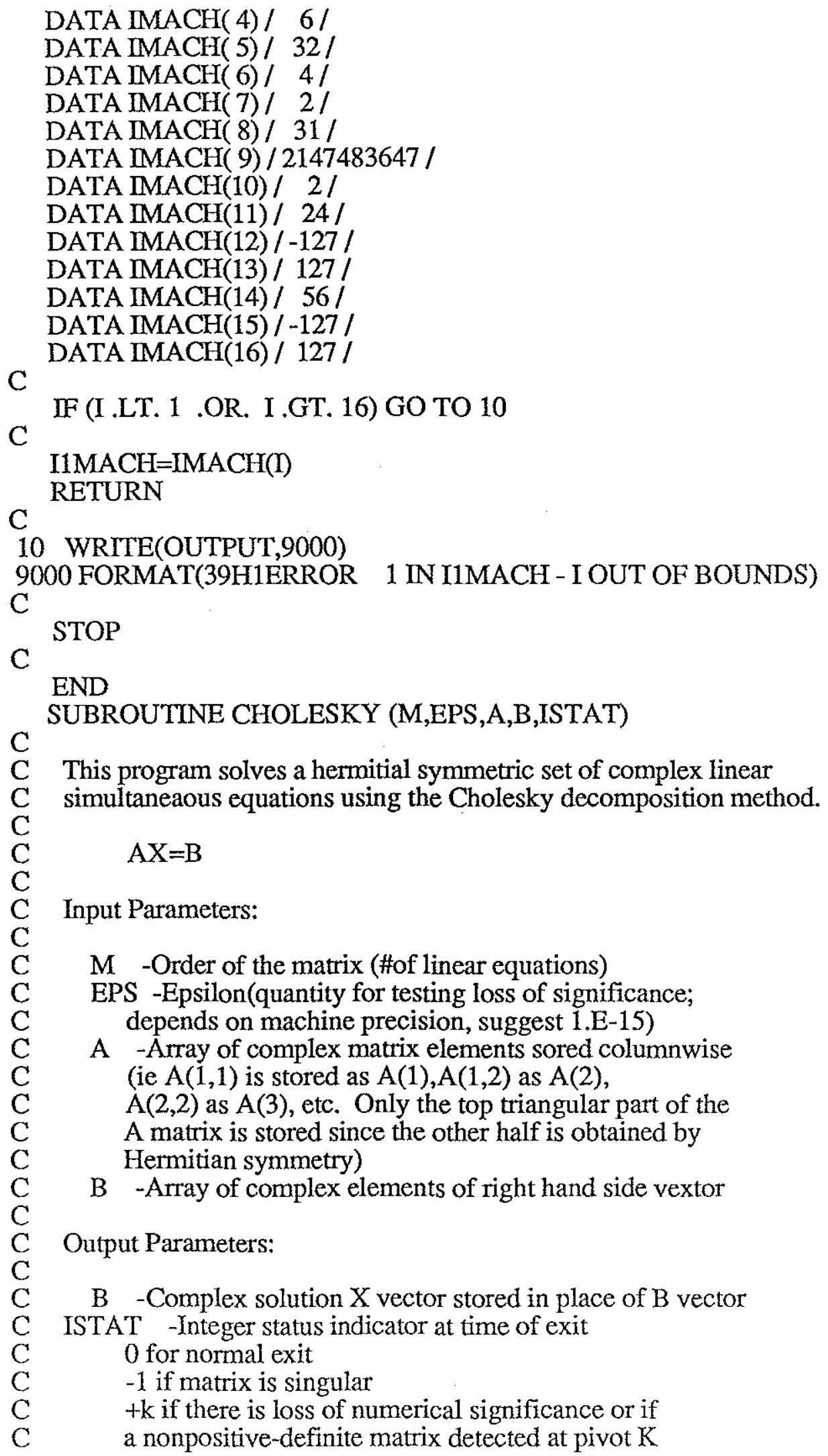


$\mathrm{C}$

C Notes:

C

C External array A must be dimensioned .GE. $M(M+1) / 2$ and array

C B must be dimensioned .GE. $M$ in the calling program. Array A

C is destroyed when this routine is called.

C

IMPLICIT DOUBLE PRECISION (A-H,O-Z)

COMPLEX*16 A(1),B(1),SUM

REAL EPS

$\mathrm{C}$

C Factor into triangular and diagonal form

C

$$
\text { DEPS }=\text { DBLE(EPS) }
$$

ISTAT $=0$

$\mathrm{KPIV}=0$

DO $100 \mathrm{~K}=1, \mathrm{M}$

$\mathrm{KPIV}=\mathrm{KPIV}+\mathrm{K}$

IND $=$ KPIV

$\mathrm{LEND}=\mathrm{K}-1$

TINY $=$ DABS(DEPS $*$ DREAL $($ A(KPIV)))

DO $100 \mathrm{I}=\mathrm{K}, \mathrm{M}$

SUM $=(0 . \mathrm{D} 0,0 . \mathrm{D} 0)$

IF (LEND .EQ. 0) GOTO 40

LPIV $=\mathrm{KPIV}$

DO $30 \mathrm{~L}=1, \mathrm{LEND}$

$\mathrm{LPIV}=\mathrm{LPIV}+\mathrm{L}-\mathrm{K}-1$

30

40

SUM=SUM+DREAL(A(LPIV))*A(IND-L)*DCONJG(A(KPIV-L)) SUM=A(IND)-SUM

IF (I .NE. K) GOTO 80

$\mathrm{C}$

C Test for negative pivot element and loss of significance

C

IF (DREAL(SUM) .GT. TINY) GOTO 90

IF (DREAL(SUM) .GT. 0.D0) GOTO 70

ISTAT $=-1$

RETURN

70 IF (ISTAT .GT. 0) GOTO 90

ISTAT $=K$

90 A(KPIV) $=$ DCMPLX(DREAL(SUM),0.D0)

DPIV $=(1 . D 0) /$ REAL(SUM)

GOTO 100

$80 \quad \mathrm{~A}(\mathrm{IND})=\mathrm{SUM} * \mathrm{DPIV}$

$100 \quad \mathrm{ND}=\mathrm{IND}+\mathrm{I}$

C

C Solve for intermediate column vector solution

C

$\mathrm{KPIV}=1$

DO $200 \mathrm{~K}=2, \mathrm{M}$

$\mathrm{KPIV}=\mathrm{KPIV}+\mathrm{K}$

SUM $=\mathrm{B}(\mathrm{K})$

DO $210 \mathrm{~J}=1, \mathrm{~K}-1$ 


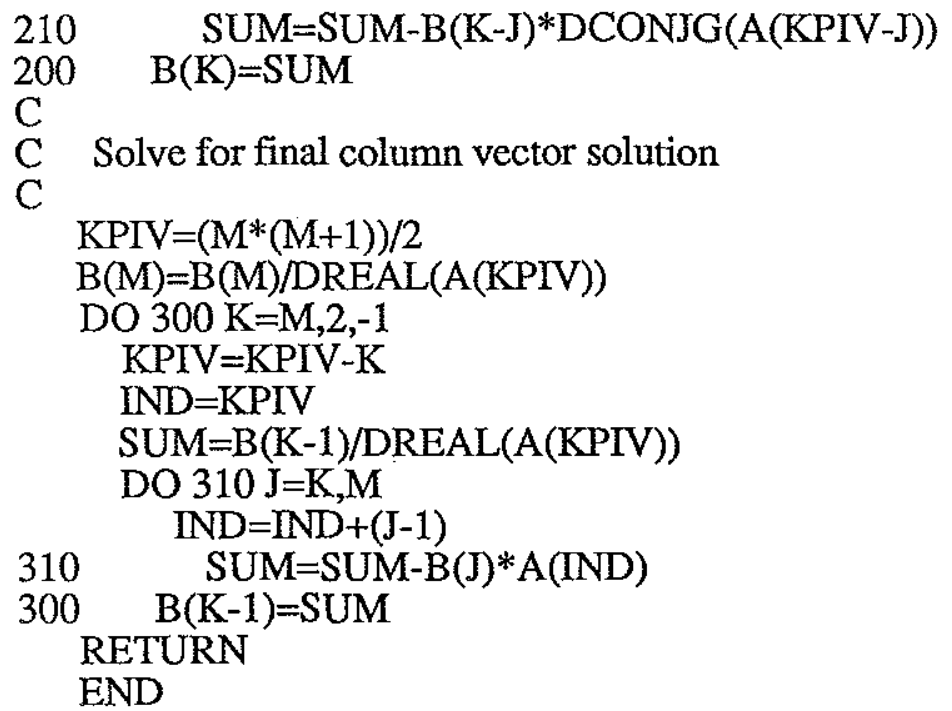




\section{Bibliography}

[1] . F.D. DiNapoli. Fast Field Program for Mulilayered Media. Technical Report NUSC 4103, Naval Underwater Systems Center, New London, Connecticut, August 1971.

[2] A.L. Kurkjian. Numerical Computation of Individual Far-Field Arrivals Excited by an Acoustic Source in a Borehole. Geophysics, 50(5):852-866, May 1985.

[3] D.H. Trivett and A.Z. Robinson. Modified Prony Method Approach to EchoReduction Measurements. Journal of Acoustical Society of America. 70(4):1166-1175, October 1981.

[4] L.C. Chang, D.L. Moffattt, L. Peters, Jr. Estimation of Complex Natural Resonances of a Class of Subsurface Targets in V.K. Varadan and V.V Varadan, editors Acoustic Electromagnetic and Elastic Wave Scattering - Focus on T Matrix Approach.Pergamon Press, 1980.

[5] H.P. Bucker. Comparision of FFT and Prony Algorithms for Bearing Estimation of Narrowband Signals. Journal of the Acoustical Society of America. 61(3):756-762, March 1977.

[6] S. Braun and Y.M. Ram. Determination of Structural Modes Via the Prony Model: System Order and Noise Induced Poles. Journal of the Acoustical Society of America, 81(5):1447-1459, May 1987.

[7] E.C. Shang, H.P Wang and Z.Y. Haung. Waveguide Characterization and Source Localization in Shallow Water Waveguides using the Prony Method. 112th Meeting, Acoustical Society of America, Anaheim, CA, Dec 1986.

[8] J.F. Lynch, S.D. Rajan, and G.V. Frisk. Perturbative Inversion Methods for Obtaining Bottom Geoacoustic Parameters in Shallow Water. to be published in the Journal of the Acoustical Society of America. (Sep 1987).

[9] G.V. Frisk, A.V. Oppenheim, and D. R. Martinez. A Technique for measuring the Plane-Wave Reflection Coefficient of the Ocean Bottom. Journal of the Acoustical Society of America, 68(2), August 1980.

[10] G.V. Frisk, J.F. Lynch and J.A. Doutt. The Determination of Geoacoustic Models in Shallow Water. In T. Akal and J.M. Berkson, editors, Ocean Seismo-acoustics, Plenum Publishing corporation, 1986.

[11] S.D. Rajan. An Inverse Method for Obtaining the Attenuation Profile and Small Variations in the Sound Speed and Density Profiles of the Ocean Bottom.ScD Thesis, MIT/WHOI Joint Program, WHOI 85-30, May 1985.

[12] J.E. Matthews, P.J. Bucca, and W.H. Geddes, Preliminary Environmental Assessment of the PROJECT GEMINI site - Corpus Christi, Texas. NORDA Report 120, Naval Ocean Research and Deveopment Activity, Bay St. Louis, MS, June 1985.

[13] G.V. Frisk and J.F. Lynch. Shallow Water Waveguide Characterization Using the Hankel Transform. Journal of the Acoustical Society of America, 76(1):205-216, July 1984. 
[14] C.A. Boyles, Acoustic Waveguides. John Wiley and Sons, New York, 1984.

[15] F.B. Hildebrand. Advanced Calculus for Applications. Prentice-Hall, Inc., Englewood Cliffs, NJ, 1976.

[16] E.C. Titchmarsh. Eigenfunction Expansions. Oxford University Press, London, 1946.

[17] C.L Bartberger. Comparsion of Two Normal-Mode Solutions Based on Different Branch Cuts.Journal of the Acoustical Society of America, 61:1643, 1977.

[18] H.P. Bucker. Propagation in a Liquid Layer Lying Over a Liquid Half-Space (Pekeris Cut). Journal of the Acoustical Society of America, 65(4):906-908, April, 1979.

[19] A.O. Williams. Pseudoresonances and Virtual Modes in Underwater Sound Propagation.Journal of the Acoustical Society of America, 64(5):1487-1491, Nov., 1978.

[20] C.T. Tindle, A.P. Stamp, and K.M. Guthrie. Virtual Modes and the Surface Boundary Condition in Underwater Acoustics. Journal of Sound Vibration, 49:231-240, 1976.

[21] M. Abramowitz and I. Stegun. Handbook of Mathematical Functions. National Bureau of Standards, 1964.

[22] J.J. Polcari. Acoustic Mode Coherence in the Artic Ocean. ScD Thesis, MIT/WHOI Joint Program, WHOI 86-38, September 1986.

[23] C.L. Pekeris. Theory of Propagation of Explosive Sound in Shallow Water. Geological Society of America, Memoir 27, 1948.

[24] George V. Frisk, private communication

[25] M.S. Wengrovitz, The Hilbert-Hankel Transform and its Application to Shallow Water Ocean Acoustics. Technical Report 513, ScD Thesis, MIT/WHOI Joint Program, Cambridge, MA, January 1986.

[26] C.S. Clay and H. Medwin. Acoustical Oceanography. John Wiley \& Sons, New York, 1977.

[27] L.M. Brekhovshikh. Waves in Layered Media. Academic Press, New York, 1980.

[28] I.Tolstoy and C.S.Clay. Ocean Acoustics. McGraw-Hill Book Company, New York, 1966.

[29] J. Makhoul. Spectral Linear Prediction: Properies and Applications. Institute for Electrical and Electronic Engineers Transactions on Acoustics, Signals and Speech Processing., ASSP-23(3):283-296, June 1975.

[30] F.B. Hildebrand, Introduction to Numerical Analysis, McGraw-Hill, NewYork, 1956.

[31] S.L. Marple Jr. Digital Spectral Analysis with Applications. Prentice-Hall, 1987. 
[32] S.M. Kay and S.L. Marple. Spectrum Analysis - A Modern Perspective. Proceedings of the Institute of Electrical and Electronic Engineers., 61(5):1380-1419, May 1977.

[33] J. Makhoul. Linear Prediction: A Tutorial Review. Proceedings of the Institute of Electrical and Electronic Engineers, 63(4):561-580, April 1975.

[34] J. Makhoul. Stable and Efficient Lattice Methods for Linear Prediction. Institute for Electrical and Electronic Engineers Transactions on Acoustics, Signals and Speech Processing., ASSP-26(3):423-428, February 1978.

[35] J.H. McClellan. Two-Dimensional Spectrum Analysis in Sonic Logging. Institute of Electrical and Electronic Engineers Acoustics, Signals and Speech Processing Magazine. pp 12-18.July 1986.

[36] R. Kumaresan, D.W. Tufts, and L.L. Scharf. A Prony Method for Noisy Data: Choosing the Signal Components and Selecting the Order in Exponential Signal Models. Proceedings of the Instittue for Electronic and Electical Engineers. $72(3): 230-232$, February 1984.

[37] J.P.Burg. Maximum Entropy Spectral Analysis in Proceedings of the 37th Meeting of the Society of Exploration Geophysicists, Okalhoma City, OK, October 1967.

[38] J.P. Burg. Maximum Entropy Spectral Analysis, PhD Dissertation, Department of Geophysics, Stanford University, Stanford, CA, May 1975.

[39] C.L. Lawson and R.J. Hanson. Solving Least Square Problems. PrenticeHall,Englewood Cligffs, NJ, 1974.

[40] G.H. Goulub and C.F. Van Loan. Matrix Computations. Johns Hopkins University Press, Baltimore, MD, 1983.

[41] J.H. Wilkinson. The Algebraic Eigenvalue Problem. Claredon Press, Oxford, 1965.

[42] H. Akaike, A New Look at Statistical Model Identification. Institute of Electrical and Electronic Engineer Transactions on Automated Controls. AC19:716-723, Mar 1967.

[43] R. Kumaresan and D.W. Tufts. Estimating the Parameters of Exponenetially Damped Sinusoids and Pole-Zero Modeling Noise. Institute for Electrical and Electronic Engineers Transactions on Acoustics, Signals and Speech Processing., ASSP-30(6):833-840, December 1982.

[44] D.W. Tufts and R. Kumaresan. Singular Value Decomposition and Improved Frequency Estimation Using Linear Prediction. Institute for Electrical and Electronic Engineers Transactions on Acoustics, Signals and Speech Processing. ASSP 30:671-675, August 1982.

[45] M.A. Jenkins and J.F. Traub. Algorithm 419: Zeroes of a Complex Polynomial. Association of Computing Machinery, 15:917-919, February 1972.

[46] G. Strang. Introduction to Applied Mathmatics. Wellesley-Cambridge Press, 1986.

[47] F.B. Jensen and M.C. Ferla. SNAP: The SACLANTEN Normal-Mode Acoustic Propagation Model. Report SM-121, SACLANT Research Centre, La Spezia, Italy, 1979. 
[48] D.R. Mook, G.V. Frisk, and A. V. Oppenheim. A Hybrid Numerical/Analytic Technique for the Computation of Wave Fields in Stratified Medial Based on the Hankel Transform. Journal of the Acoustical Society of America, 76(1):222-243, July 1984.

[49] A.V. Oppenheim, G.V. Frisk, and D.R. Martinez. Computation of the Hankel Transform Using Projections. Journal of the Acoustical Society of America, 68(2):523-529, August 1980.

[50] D.R Mook. The Numerical Synthesis and Inversion of Acoustic Fields Using the Hankel Transform with Application to the Estimation of the Plane Wave Reflection Coefficient of the Ocean Bottom. Technical Report, Sc.D. Thesis, MIT/WHOI Joint Program, Cambridge MA, Jan. 1983.

[51] A.V. Oppenheim and R.W. Schafer. Digital Signal Processing. Prentice-Hall, Inc., Englewood Cliffs, New Jersey, 1975.

[52] R. Kumaresean and D.W. Tufts. Improved Spectral Resolution M: Efficient Realization. Proceedings of the Institute of Electrical and Electronic Engineers. 68(5), May 1977.

[53] D.W. Tufts and R. Kumaresan. Improved Spectral Resolution. Proceedings of the Institute of Electrical and Electronic Engineers. 68(3):419-421, March 1980.

[54] D.W. Tufts and R. Kumaresan. Estimation of Frequency of Multiple Sinusoids: Making Linear Prediction Perform like Maximum Likelihood. Proceedings of the Institute of Electrical and Electronic Engineers. 70:975-989, September 1982.

[55] H. Schmidt and F.B. Jensen. A Full Wave Solution for Propagation in Multilayered Viscoelastic Media with Application to Gaussian Beam Reflection at Fluid-Solid Interfaces. Journal of the Acoustical Society of America. 77(3):813-825, March 1985.

[56] F.Gilbert and G.E Bakus. Propagation Matricies in Elastic Wave and Vibration Problems. Geophysics, 31:326-332, 1966.

[57] IMSL library, version 8. International Mathmatical and Statistical Libraries Inc., Houston, TX.

[58] R.N. McDonough. Representation and Analysis of Signals, Part XV, Matched Exponentials for the Representation of Signals, Johns Hopkins University Department of Electrical Engineering Report, Baltimore, MD, April 1963.

[59] S.T. McDaniel. Mode Coupling Due to Interaction with the Seabed. Journal of the Acoustical Society of America, 72:916-923, 1982.

[60] L.G. Beatty, J.D. George and A. Z. Robinson. Use of the Complex Exponential Expansion as a Signal Representation for Underwater Acoustic Calibration. Journal of the Acoustical Society of America, 63:1782-1794, 1978.

[61] A.D. Pierce. Extension of the Method of Normal Modes to Sound Propagation in an Almost-Stratified Medium. Journal of the Acoustical Society of America, 37(1):19-27, 1965. 
[62] L.M. Brekhovshikh and Y. Lysanov. Fundamentals of Ocean Acoustics. SpringerVerlag, Berlin, 1982.

[63] R.B. Evans. A Coupled Mode Solution for Acoustic Propagation in a Waveguide with Stepwise Depth Variations of a Penetrable Bottom. Journal of the Acoustical Society of America. 74(1):188-195, July 1983.

[64] F.D. Tappert. The Parabolic Equation Method. In J.B. Keller and J.S. Papadakis, editors, Wave Propagation and Underwater Acoustics, Springer-Verlag, New York, 1977.

[65] F.D. Tappert, Selected Applications of the Parabolic Equation Method in Underwater Acoustics. Internal Workshop on Low-Frequency Propagation and Noise (Woods Hole, MA), October 1974.

[66] F.B Jensen and W.A. Kuperman. Sound Propagation in a Wedge-shaped Ocean with a Penetrable Bottom. Journal of Acoustical Society of America. 67(5):1564-1566, May 1980.

[67] F.M. Labianca. Normal Modes, Virtual Modes, and Alternative Representations in the Theory of Surface Duct Sound Propagation. Journal of the Acoustical Society of America, 53:1137-1147, 1973.

[68] G.V. Frisk, F.J. Diemer and P.H. Dahl, Characterization of Range-Dependent Shallow Water Waveguides. 112th Meeting, Acoustical Society of America, Anaheim, CA, Dec 1986.

[69] E.L. Hamilton. Geoacoustic Modeling of the Sea Floor. Journal of the Acoustical Society of America. 68(5):1313-1340, November 1980.

[70] PORT Mathematical Subroutine Library. P.A. Fox, Editor, Bell Telephone Laboratry, 1976. 